\title{
INL Annual Report FY 2014 LDRD Project Summaries
}

\author{
Dena Tomchak and Heather Rohrbaugh
}

\author{
February 2015
}

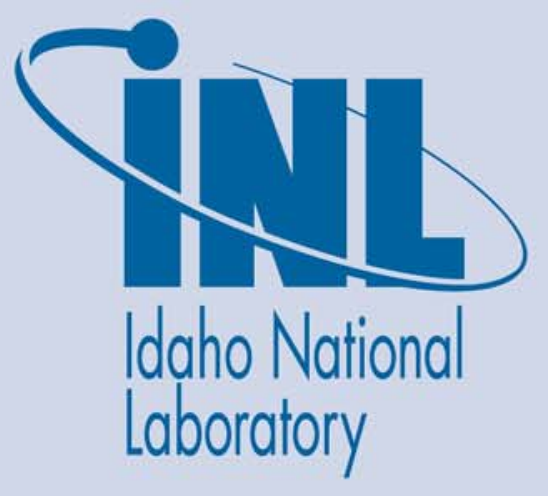

The INL is a U.S. Department of Energy National Laboratory operated by Battelle Energy Alliance 
INL/EXT-14-33589

\title{
INL Annual Report FY 2014 LDRD Project Summaries
}

\author{
Dena Tomchak and Heather Rohrbaugh
}

February 2015

\section{Idaho National Laboratory \\ Idaho Falls, Idaho 83415}

http://www.inl.gov

Prepared for the

U.S. Department of Energy

Office of Nuclear Energy

Under DOE Idaho Operations Office

Contract DE-AC07-05ID14517 
Idaho National Laboratory ANNUAL REPORT FY 2014

\section{LDRD PROJECT SUMMARIES}
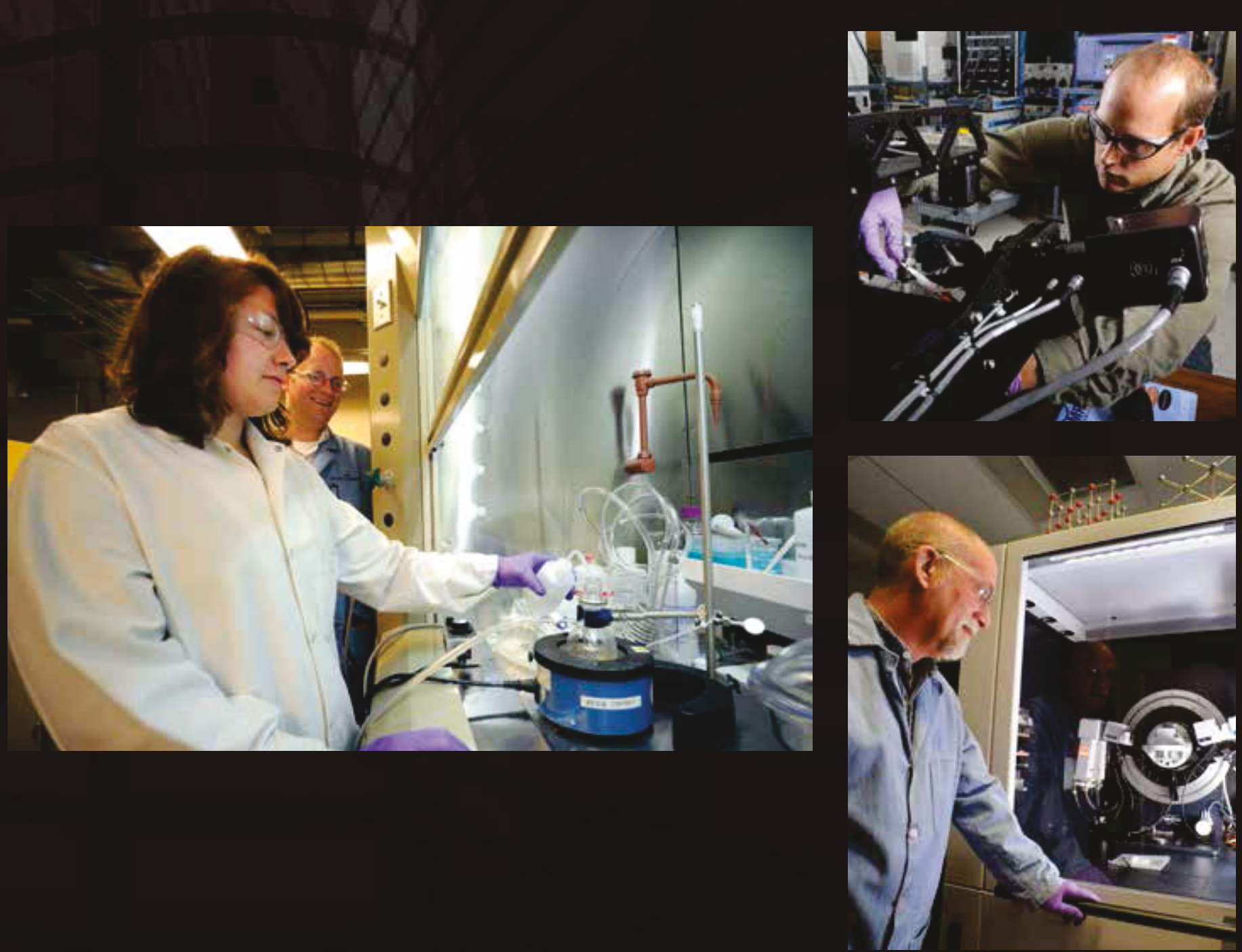

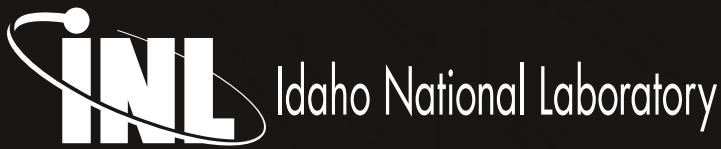




\title{
Acknowledgments
}

This annual report provides an overview of the Laboratory Directed Research and Development (LDRD) Program at Idaho National Laboratory (INL). Summarized in this report are 68 technical research projects conducted in FY 2014. The LDRD Program at INL is managed for the Laboratory by John J. Grossenbacher, Laboratory Director; Todd Allen, Deputy Laboratory Director for Science and Technology; and Anita Gianotto, LDRD Program Manager.

Many people contributed to this report; however, particular thanks go to the principal investigators who conduct the cutting-edge research so important to this laboratory.

\author{
Managing Editor: Dena Tomchak \\ Technical Writing and Heather Rohrbaugh \\ Editing: Riley Goodwin \\ Text Processing: Andrea Stringham \\ Cover Design: Kris Burnham
}

Prepared for the U.S. Department of Energy

Director, Nuclear Energy, Science \& Technology

Under DOE Idaho Operations Office

Contract Number: DE-AC07-05ID14517

\begin{abstract}
Disclaimer
This report was prepared as an account of work sponsored by an agency of the United States Government. Neither the United States Government nor any agency thereof, makes any warranty, express or implied, or assumes any legal liability or responsibility for the accuracy, completeness, or usefulness of any information, apparatus, product, or process disclosed, or represents that its use would not infringe privately owned rights. Reference herein to any specific commercial product, process, or service by trade name, trademark, manufacturer, or otherwise does not necessarily constitute or imply its endorsement, recommendation or favoring by the United States Government or any agency thereof. The views and opinions of authors expressed herein do not necessarily state or reflect those of the United States Government or any agency thereof.
\end{abstract}




\section{CONTENTS}

Section 1 Nuclear Science and Technology. .1

12-031 - 2-D and 3-D EBSD Technique Development and Microstructure Reconstruction for Phase Field

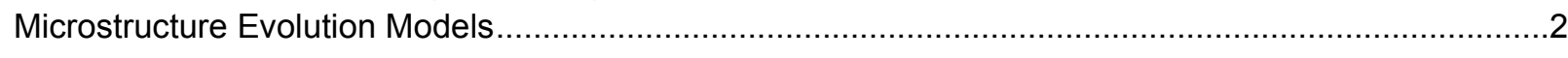

12-061 — Development of a High Burnup Zr-based Fuel Cladding for LWRs...............................................4

12-062 - Development of Diamond Based Sensors for User Facility Capabilities at INL Advanced Test Reactor .6

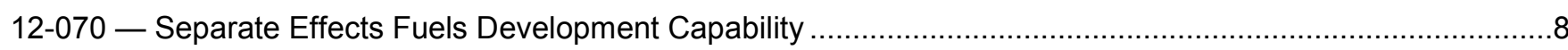

13-013 - Development of a Micromechanistic Phase-Field Modeling Approach for Life Estimation and Risk Assessment of Reactor Pressure Vessels ............................................................................... 10

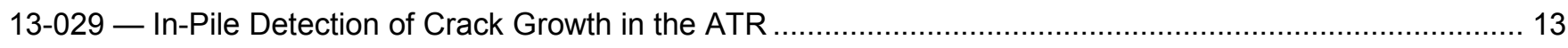

13-032 - Experimental and Computational Analysis of Hydride Microstructures in Zirconium in Dry Storage

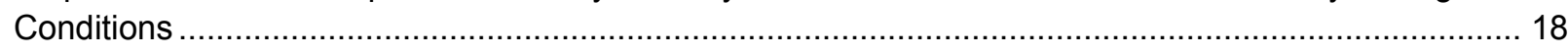

13-039 - Induction Based Fluidics Mass Spectrometry for Characterizing Radioactive Extraction Solvents ....... 20

13-040 - Epistemic Uncertainty Quantification in Dynamic Probabilistic Risk Assessment.............................. 22

13-050 - Concurrent Atomistic to Macroscale Modeling of Materials under Irradiation Using the Phase Field

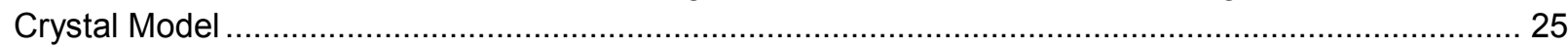

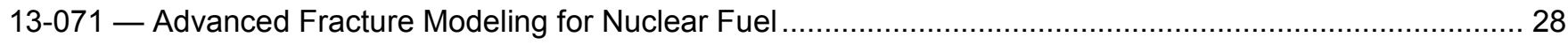

13-097 — MOOSE Capability Extension In Support of Full Core Modeling.................................................. 31

13-106 - Building Inorganic-Organic Hybrid Materials to Protect Metal Ion Sequestering Agents from

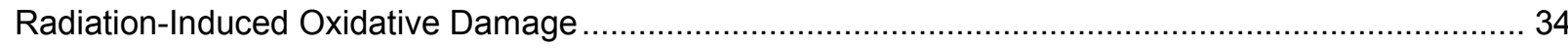

13-115 - Multi-scale Full Core Reactor Physics Simulation of the Advanced Test Reactor ............................ 36

13-121 - Advanced In-Situ Measurement Techniques in TREAT ............................................................. 39

14-009 - Development of an INL Capability for High Temperature Flow, Heat Transfer, and Thermal Energy Storage with Applications in Advanced Small Modular Reactors, High Temperature Heat Exchangers, Hybrid Energy Systems, and Dynamic Grid Energy Storage Concepts ........................................... 41

14-010 - Use of Linear Variable Differential Transformer (LVDT)-Based Methods to Detect Real-Time Geometry

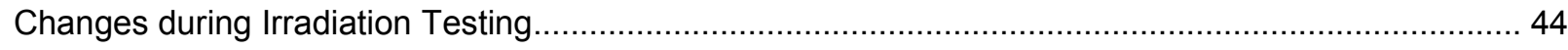

14-025 - Minor Actinide and Lanthanide Separations in Alternative Media .................................................. 47

14-026 — Multiscale Modeling on Delayed Hydride Cracking in Zirconium: Hydrogen Transport and Hydride

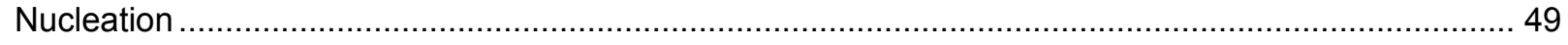

14-031 - Multidimensional Multiphysics Modeling of Fuel Behavior During Accident Conditions..................... 52

14-038 - Advanced Automated Validation of Modeling and Simulation Tools .............................................. 55

$14-041$ - Fabrication of $\mathrm{UN}-\mathrm{U}_{3} \mathrm{Si}_{2}$ Composites via Spark Plasma Sintering ................................................ 57

14-075 - Development of Tools and Methodologies for Uncertainty Quantification and Validation for Multiphysics

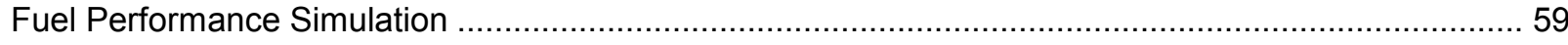


14-098 — Irradiation Effects in Uranium Dioxide ..... 64

14-102 - Advanced Seismic Probabilistic Risk Assessments ...... 67

14-104 - Development of a Multiphysics Algorithm for Analyzing the Integrity of Nuclear Reactor Containment Vessels Subjected to Extreme Thermal and Overpressure Loading Conditions

Section 2 National and Homeland Security .74

12-001 - Cyber Security for 4th Generation Cellular Communications. 75

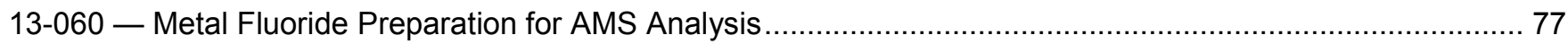

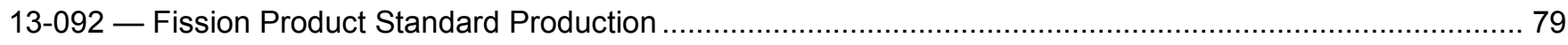

13-093 - Spectrum Allocation and Communications in Dynamic Spectrum Access Channels ........................ 82

13-118 - Geomagnetic Disturbance Field Coupling Measurements on INL Power Grid ................................ 85

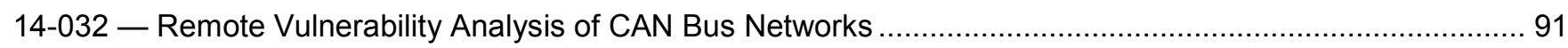

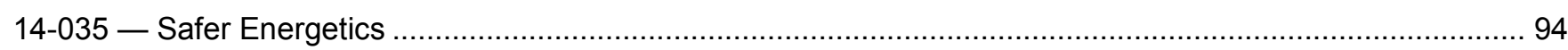

14-036 — Innovative Research for Fieldable Nuclear Measurements........................................................ 96

14-037 - Advanced Nuclear Material Characterization Technology for Security Applications........................... 98

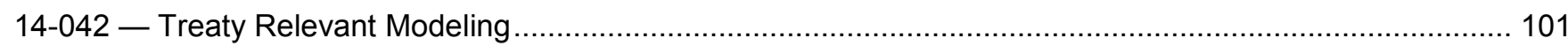

14-044 — Development of Halide and Oxy-Halides for Isotopic Separations.............................................. 105

14-045 — End-to-End Radiation Detector Enhancements for Improved Safety and Security in Safeguarded

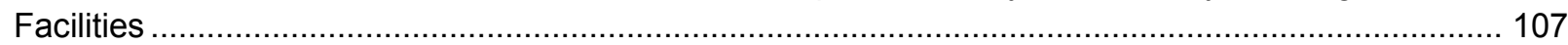

14-087 - Transparent Fiber Reinforcements for Transparent Protection Systems ..................................... 109

14-090 — SWANS: Secure Wireless Sensor Networks for Smart Grid Communications ............................... 111

14-093 - All Hazards Critical Infrastructure Knowledge Framework ........................................................ 113

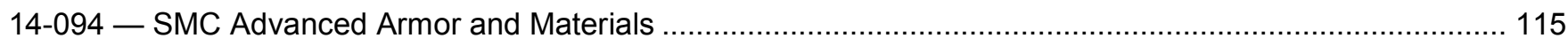

14-103 — Grid-Centric Demonstration of Resilience Technologies........................................................ 117

SECTION 3 ENERGY \& ENVIRONMENT SCIENCE AND TECHNOLOGY ..................................................................120

12-093 - Development of Unique Nanomaterials for Selective Replacement of Strategic and Critical Materials

12-113 - Coupled-Smoothed Particle Hydrodynamics and Discrete Element Model (SPH-DEM) Simulation of Hydraulic Fracturing and Shale Gas Production ..................................................................... 123

13-027 — Diagnostics of Advanced Energy Storage Materials ........................................................... 127

13-065 - Multi-Domain Modeling, Simulation, and Integration Tools - Hybrid Energy Systems ..................... 130

13-079 — Diverse Biological Factories for Sustainable Manufacturing ................................................... 133

13-114 - Multiphase Fluid Trapping in Propped Hydraulic Fractures .................................................... 135

14-078 — Extended Stability Gamma-Gamma Prime Containing Nickel-Base Alloys ................................. 138 
14-079 — Second Generation Switchable Polarity Solvent Draw Solutes for Forward Osmosis ...................... 140

14-080 — Battery Material Characterization Technologies.................................................................. 143

14-086 — Development of a Microgrid/Smartgrid Testbed for ESL and Super Lab Initiative with Load Variability Characterization and Control for Renewable Energy Integration ................................................ 149

14-091 - Battery Health Estimation Based on Self-Discharge Fast Measurement and Soft Short-Circuit Detection

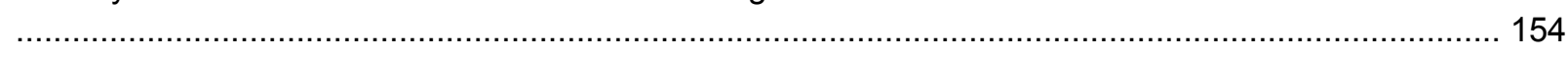

14-095 — In Situ Measurement of Electrolyte Chemistry in Battery Cells During Operation ........................... 157

14-106 — Understanding the Growth of Ultra-Long Carbon Nanotubes .................................................. 159

SECTION 4 INSTITUTE FOR NUCLEAR SCIENCE AND TECHNOLOGY ..............................................................162

12-026 - Protectiveness and Stability of the Zirconium Oxide in Early-Phase Corrosion of Zirconium Alloys Predictive Relations to Surface Structure and Composition ......................................................... 163

12-056 - Recovery of Precious Metals from Used Nuclear Fuel and Electronic Waste Using Sulfur-Impregnated

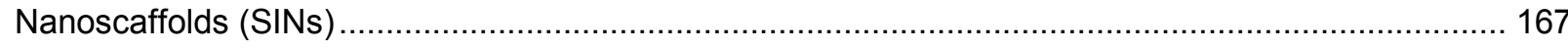

$12-059$ - Chemistry and Flowsheet Development for Lanthanide Separations …..................................... 170

12-077 - Observation of Zirconium Oxidation at Atomic Level Using Non-Linear Optical Spectroscopy .......... 172

13-095 - Development and Validation of a Societal-Risk Goal for Nuclear Power Plant Safety ...................... 176

13-105 - Micro/Nano Scale AFM-based Thermal Conductivity Measurement and Atomistic Modeling for Oxide Fuel: The Effects of Grain Boundary, Fission Gas and Radiation Damage .................................. 178

13-110 — Nuclear-Renewables-Oil Shale Hybrid Energy System .......................................................... 181

SeCtion 5 Center for AdVANCEd ENergy StUdies ..........................................................................184

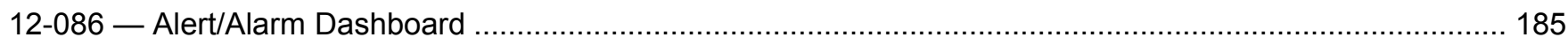

13-011 — Integrated Approach to Algal Biofuel, Bio-power, and Agricultural Waste Management ................... 189

13-033 - Magnetic Separation Nanotechnology for Spent Nuclear Fuel Recycle ....................................... 193

13-035 - Development of New Molten Salt Sensor Technology for Application to Safeguarding Pyroprocessing

13-068 - Cooling in Fractured Geothermal Reservoirs: Analyses of Long-Term Cooling in Typical Geothermal Reservoirs and Application to Geothermal Resource Potential Assessments ................................. 200

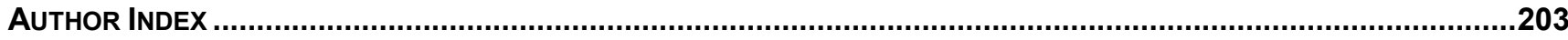




\section{NUCLEAR SCIEN Gañ̄ TECHNOLoor}




\title{
12-031 - 2-D and 3-D EBSD Technique Development and Microstructure Reconstruction for Phase Field Microstructure Evolution Models
}

\author{
Melissa C. Teague, Michael Tonks, Stephen Sintay, ${ }^{1}$ and Brian Gorman ${ }^{2}$
}

Electron back-scatter diffraction (EBSD) scans provide a detailed description of a microstructure that can be reconstructed to define initial microstructures for mesoscale models. While reconstruction methods exist for collecting EBSD scans from non-radiological samples, method development is needed to perform these types of experiments on radiological samples. This project aims to develop a method to reconstruct realistic microstructures from EBSD scans of un-irradiated and irradiated $\mathrm{UO}_{2}$ and Zircaloy cladding for use in INL's finite element-based phase field code MARMOT. Additionally, no current method exists for utilizing these reconstructed 2-D and 3-D microstructures in phase field models, a deficiency that will be addressed by this LDRD. The project will develop experimental techniques for collecting 2-D and 3-D EBSD data from radiological samples and the methodology to reconstruct and import this data directly into mesoscale phase field models.

\section{Summary}

The focused ion beam, along with its attached EBSD detector, was used to characterize the 3-D microstructure and chemistry of irradiated oxide fuel. Both the ability to serial section and perform EBSD on irradiated fuel are unique, first-of-their-kind research performed at INL, and several journal articles are expected from this work. Having the ability to study the crystal structure, chemistry, and microstructure of irradiated fuel in 3-D is critical to understanding the fundamentals of fuel performance.

The framework was developed to reconstruct 2-D structural data obtained from these experiments for use in INL's mesoscale code MARMOT. Preliminary models of fission gas evolution have been developed using the reconstructed microstructure.
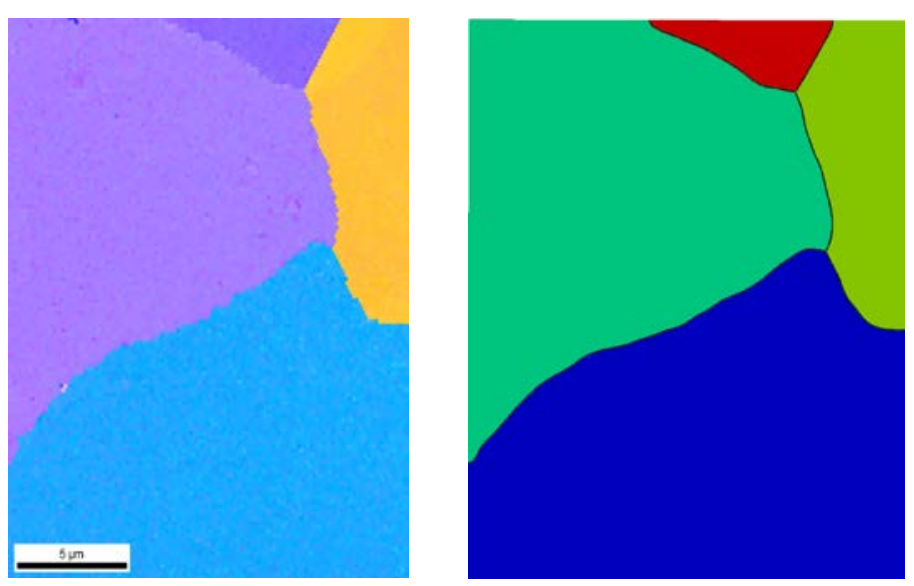

Figure 1. Microstructure reconstruction in MARMOT of a high burnup mixed oxide fuel where the EBSD scan is shown on the left and the reconstruction is shown on the right.

\footnotetext{
${ }_{2}^{1}$ Los Alamos National Laboratory

${ }^{2}$ Colorado School of Mines
} 
A Ph.D. student, Brad Fromm, is in the process of writing his Ph.D. dissertation of which this LDRD has supported the bulk of the research. Currently, three papers have been published related to this work, and another four are anticipated in FY 2015.

\section{Benefits to DOE}

DOE benefits from the project by the development of a unique, industry leading capability to analyze radiological materials utilizing 2- and 3-D EBSD as well as the ability to utilize this experimental information in phase field models. The capability to examine radiological samples with advanced characterization techniques such as EBSD is critical to understanding current fuel performance and behavior and spent nuclear fuel storage. The ability to reconstruct the experimental data and utilize it in phase field modeling efforts provides critical synergy between experimental efforts and lower length-scale modeling.

\section{Publications}

Teague, M., B. Gorman, B. Miller, and J. King, "EBSD and TEM Characterization of High Burn-Up Mixed Oxide Fuel," Journal of Nuclear Materials, Vol. 444, 475-480, 2014.

Teague, M., and B. Gorman, "Utilization of Dual-Column Focused lon Beam and Scanning Electron Microscope for Three Dimensional Characterization of High Burn-Up Mixed Oxide Fuel," Progress in Nuclear Energy, Vol. 72, 67-71, 2014.

Teague, M., B. Fromm, M. Tonks, and D. Field, "Using Coupled Mesoscale Experiments and Simulations to Investigate High Burn-Up Oxide Fuel Thermal Conductivity," JOM Journal of the Minerals Metals and Materials Society, Vol. 66, 2569-2577, 2014.

\section{Presentations}

Fromm, B., M. Tonks, M. Teague, and D. Field, "Microstructure Reconstruction Methods for Modeling Nuclear Materials," EBSD 2014, Pittsburgh, PA, June 19, 2014.

Teague, M., B. Gorman, and B. Miller, "Utilization of FIB/SEM for 3D Characterization of High Burn-Up Mixed Oxide Fuel," European Material Research Society Spring Meeting, Strasbourg, France, May 27-31, 2013.

Tonks, M., D. Gaston, P. Millett, R. Williamson, C. Stanek, and D. Andersson, "Multiscale Fuel Performance Modeling: Coupling Atomistic, Meso- and Continuum Scale Simulations," 2012 ANS Annual Meeting, Chicago, IL, June 25-28, 2012.

Teague M., B. Gorman, S. Hayes, and J. Carmack, "Microstructural and Chemical Characterization of High Burn-up Mixed Oxide Fuel," TMS 2012, Orlando, FL, March 11-15, 2012. 


\title{
12-061 - Development of a High Burnup Zr-based Fuel Cladding for LWRs
}

\author{
Robert Mariani and James I. Cole
}

A new oxide dispersion-strengthened (ODS) zirconium ( $\mathrm{Zr}$ )-based alloy was sought for improved safety, reliability, and burnup in light water reactor applications. However, ODS alloys have high prices that arise from traditional mechanical alloying that involves energy-intensive, time-consuming, and labor-intensive processes. To be cost-effective, an ODS Zr-based cladding alloy would therefore require new materials and methods that eliminate traditional powder metallurgical methods. Novel ODS agents were conceived that could be used in a way that eliminates traditional powder metallurgy processes, such as mechanical alloying, and permit large batch and/or continuous castings.

Proposed material additions to the $\mathrm{Zr}$ alloy were based on thermodynamics, experimental data, a simple transport model, and prior modeling experience with other ODS-type materials. Nanoscale agents were designed that were expected to meet the technical and fabrication goals. In particular, nano-particles of molybdenum (Mo) coated with $\mathrm{Nd}_{2} \mathrm{O}_{3}$ were considered a viable nanoscale agent to formulate an ODS-type alloy. A vendor was identified who might be able to produce such materials, and a subcontract was issued on a best effort basis, since no like material had been hitherto prepared, beginning late in FY 2012 (the first year of the LDRD).
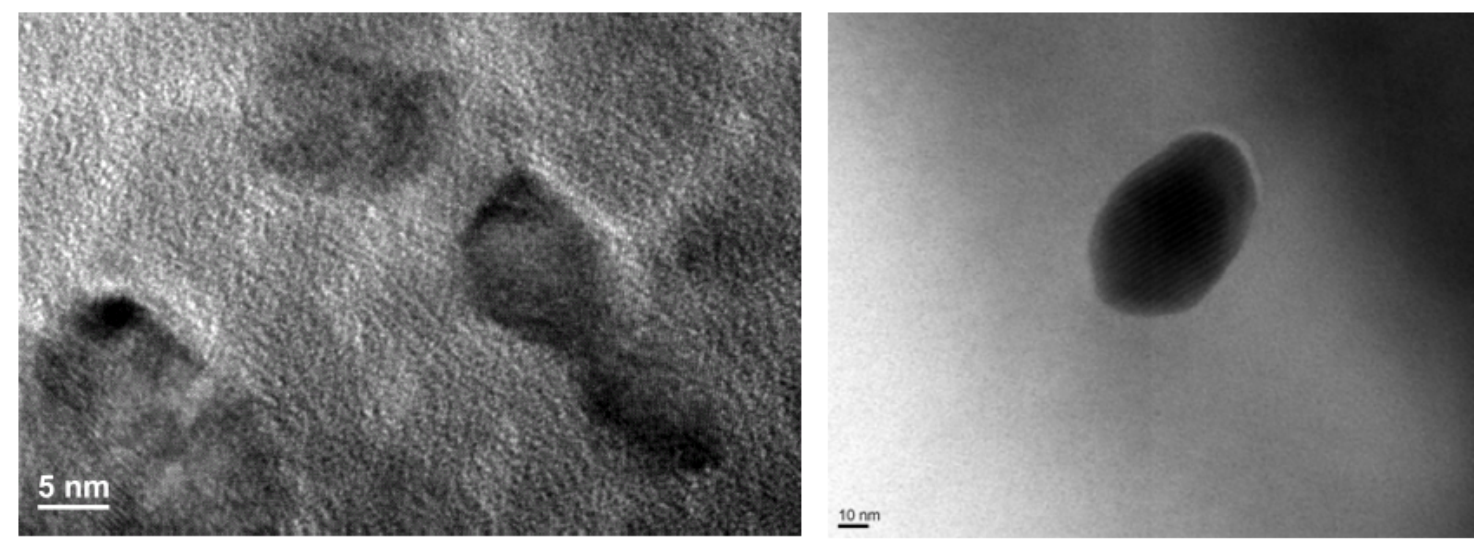

Figure 1. Transmission electron microscope images of Mo nanoparticles. Left: nanoparticles mounted in epoxy, aged in toluene approximately 6 weeks. Right: nanoparticles mounted in tin (Sn), aged approximately 3 months.

\section{Summary}

Three external publications resulted from this activity: a peer-reviewed conference publication, a patent that was issued for $\mathrm{Zr}$ alloys, and a separate patent application that was made for iron (Fe)-based alloys. Nanoscopic Mo was in fact produced, as reported in the FY 2013 annual report and illustrated in Figure 1. The overall goal to prepare new alloys, however, was not met, owing to the vendor's failure to supply quantities of coated Mo sufficient for alloy formulation. Late in the second year (FY 2013), LDRD funding was substantially withdrawn as the prospect for a follow-on subcontract with the vendor was diminished. In the third year (FY 2014), a small amount of continuing funding was used to put an existing high temperature furnace into an existing argon (Ar) glove box, thereby enabling the capability for handling high surface area, precursor, metallic materials without 
exposing them to air. Also during the latter part of the second year and all of the third year, the analysis made for developing ODS-type $\mathrm{Zr}$ alloys was applied to Fe-based alloy systems, which produced the patent application mentioned above. In particular, several Fe-based alloy compositions were explicitly identified whose formulation would prove the validity of the working model, which was developed to prepare ODS-type materials using routes more economical than those existing and to weld using conventional fusion methods.

\section{Benefits to DOE}

A novel working model for ODS-type materials was developed with application to Zr-based alloys. Owing to the lack of experimental progress stemming from novel nanoscopic production methods at a vendor, similar concepts were applied to Fe-based systems to identify distinct compositions that would validate the working model with simple, economical experiments. The scientific community is now poised to verify the applicability of those models for ODS-type steels and nanoferritic alloys, in particular. As a sidelight, a novel mechanism for the behavior of niobium ( $\mathrm{Nb}$ ) in $\mathrm{Zr}$-based cladding alloys was published. For commercial $\mathrm{Zr}$ cladding alloys, $\mathrm{Nb}$ is added to suppress surface corrosion (oxidation), but in addition, the extent of hydriding is reduced in $\mathrm{Nb}$-bearing alloys even when the extent of corrosion is comparable to that for $\mathrm{Nb}$-free alloys. In the article, the difference in the extent of hydriding was attributed to the difference in hydrogen $(\mathrm{H})$ permeability of the cladding when $\mathrm{Nb}$ is present.

\section{Publications:}

Mariani, R., "Zirconium-Based Alloys, Nuclear Fuel Rods and Nuclear Reactors Including Such Alloys, and Related Methods," U.S. Patent 8,831,166, September 9, 2014.

Mariani, R., and D. Porter, "Compositions of Particle Comprising Rare-earth Oxides in a Metal Alloy Matrix and Related Methods," U.S. Patent Application No. 14/225,306, filed March 25, 2014.

Mariani, R., J. Cole, and A. Aitkaliyeva, "A Novel Zr-1Nb Alloy and a New Look at Hydriding," Proceedings of the LWR Fuel Performance Meeting, TopFuel 2013, American Nuclear Society, Charlotte, NC, September 15-19, 2013 (peer-reviewed paper and presentation). 


\title{
12-062 - Development of Diamond Based Sensors for User Facility Capabilities at INL Advanced Test Reactor
}

\author{
David L. Chichester, James T. Johnson, Mara M. Grinder, and Scott J. Thompson
}

Chemical vapor deposited (CVD) diamond-film semiconductors can be used to simultaneously monitor intense thermal- and fast-neutron radiation fields. This project has evaluated several CVD-diamond detector sensors to assess their potential use as in-core neutron flux monitors to support the ATR NSUF research activities. These detectors operate like other semiconductor radiation detectors, but they have rather unique physical and electrical properties ideally suited for use in harsh environments. A schematic layout of a typical diamond semiconductor detector is shown in Figure 1. Ionizing radiation creates electron-hole pairs in the diamond as it traverses the material $(13 \mathrm{eV}$ per electron-hole pair); an applied high-voltage creates an

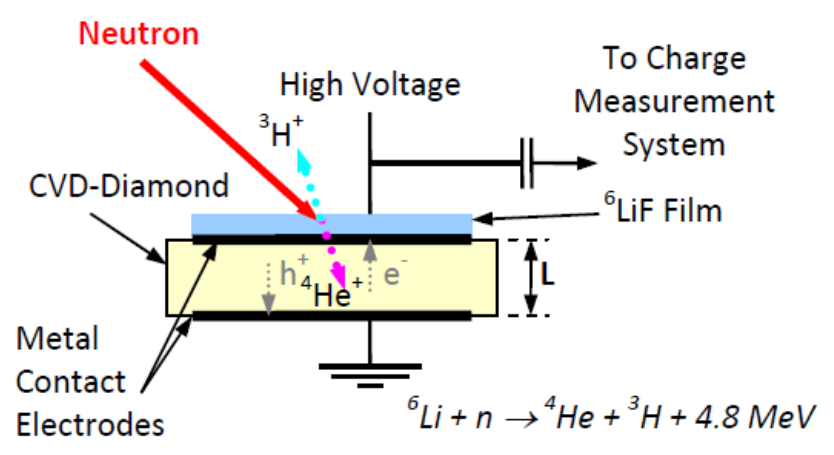

Figure 1. Schematic layout of a standard metal-insulator-metal diamond semiconductor with a ${ }^{6}$ LiF coating designed for thermal neutron detection. electric field within the crystal which sweeps the charge signals to the electrodes, resulting in a measurable current pulse. The thickness, $\mathrm{L}$, of the diamond layer can range from as thick as $0.5 \mathrm{~mm}$ to thinner than $0.05 \mathrm{~mm}$; even thinner layers are possible, although they are difficult to fabricate. Applied voltages of 500 to $1000 \mathrm{~V}$ are typical. Fast neutron detection can be made using these detectors directly as result of neutron capture in carbon (C). For thermal neutron detection, a conversion material must be used to generate an ionizing particle. Conversion film examples include lithium- 6 fluoride $\left({ }^{6} \mathrm{LiF}\right)$, polyethylene, and uranium (U)-235.

\section{Summary}

In the first 2 years of this project, four CVD-diamond detectors were evaluated. The first two units were purchased through a commercial vendor. The second two were obtained through a research collaboration with Dr. Maurizia Angelone of the Italian National Agency European Nuclear Energy Association and Rome Tor Vergata University. Fast neutron detector responses were measured using spontaneous fission isotopic sources and electronic neutron generators at INL facilities. The commercial detectors were taken from Massachusetts Institute of Technology's Nuclear Research Reactor in Cambridge, MA, to evaluate performance under high thermal neutron flux conditions. The detector response of each of these detectors was also monitored as a function of temperature.

The tests completed in the previous 2 years indicated that the CVD-diamond detectors would be able to provide multi-energy spectral information in high-flux and high-temperature environments. The primary goal of FY 2014 was to find a manufacturer capable of producing application-ready sensors and to characterize these detectors. Additionally, Monte Carlo modeling of CVD-diamond detectors under neutron irradiation was explored using MCNP6 and the Geant4 simulation toolkit. A CVD-diamond sensor was purchased from the Austrian company CIVIDEC Instrumentation and tested similarly to the previous evaluations. Figure 2 is a sample photograph from a fast neutron (14.1 MeV) irradiation of the CIVIDEC detector and the associated measured response. Efforts were also made in FY 2014 to advance the data collection methods for these types of detectors. 

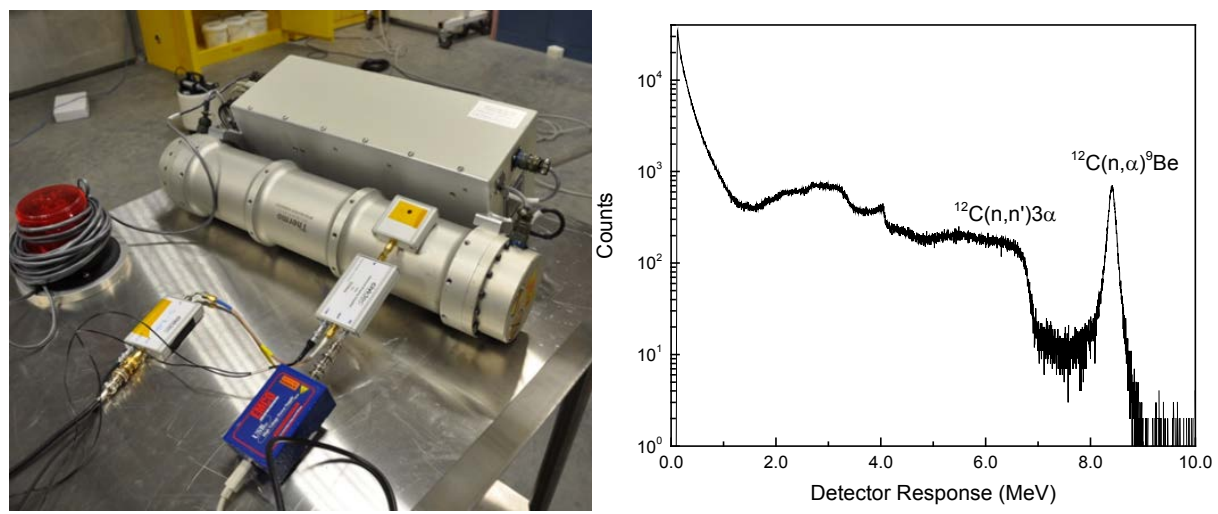

Figure 2. Photograph of the CIVIDEC CVD-diamond detector irradiated with an electronic neutron generator (left) and the recorded response from the same measurement (right).

CVD-diamond detectors have been shown to be a promising candidate for multi-energy flux monitoring inside the harsh conditions of the ATR core.

This project helped to support two graduate student interns during its lifecycle.

\section{Benefits to DOE}

Advanced thermal- and fast-neutron detection methods are directly relevant to the DOE national security mission in support of future nuclear energy systems, as described in this report. More generally, these detectors are ideally suited for use in extreme environments and, as such, may prove useful for stockpile stewardship, nuclear nonproliferation, nuclear forensics, and used fuel monitoring.

\section{Presentations}

Robinson, M., "Investigation of CVD-Diamond Detectors for Neutron Radiation Monitoring," DOE NNSA

Stewardship Science Graduate Fellowship (SSGF) Annual Program Review, Santa Fe, NM, June 25-27, 2013. 


\title{
12-070 — Separate Effects Fuels Development Capability
}

\author{
J. Keith Jewell, Tony Hill, and Eric Burgett ${ }^{1}$
}

The Separate Effects Fuels Development Capability LDRD project completed its final year of support in FY 2014. The project laid the foundation for a transformational separate effects testing program to perform in situ irradiation studies and characterization of the microscopic evolution of nuclear fuel materials under a wide variety of in-pile conditions. Separate effects testing, including fabrication, irradiation, and in situ detection of the material properties, encompasses the full science-based approach for fuels development from the nanoscale to the mesoscale behavior of the sample material. The goal was the delivery of a capability to provide an exponential increase in the available data on the time evolution of microstructural changes in fuel samples specifically engineered for isolated effects study. This data can be used in the iterative development of science-based modeling and simulation that is accurately validated and benchmarked against experimental data.

The thrust of the project was three-fold, with efforts made in all three necessary components of a complete separate effects testing capability: irradiation, detection, and fabrication of samples. Following the successful FY 2012 deliverable of an engineering design report that presented the modeling and simulation effort used to evaluate the feasibility and practicality of an accelerator driven, fuel-boosted neutron irradiation facility, focus in FY 2013 turned to the other two necessary components of a complete separate effects testing capability; advanced detection and sample fabrication.

A major focus of the FY 2013 effort was developing the advanced detection and imaging necessary for collecting the microstructure data in prototypic reactor environments. Precise, high-fidelity data at the microstructure scale is necessary for developing the phase-field modeling that describes fuel evolution during irradiation and heating cycles. However, collecting high-resolution data in extreme reactor environments presents difficulties for obvious reasons. In an effort to simulate the high neutron and radiation fields seen in a reactor, specially made samples of californium (Cf)-252 were produced on scanning electron microscope (SEM) sample stems. The deposition density of the Cf-252 was controlled such that the fission yield within the SEM field of view was comparable to typical reactor fission rates.

In FY 2014, effort was directed at fabrication of test samples specifically engineered for examining the microstructure evolution. Typical fuel sample fabrication relies on bulk processes, such as hot isostatic press and spark plasma sintering techniques designed to produce fuel pin sized quantities. The amorphous, heterogeneous composition of reactor-sized quantities makes it difficult to study the underlying microstructure that drives phenomena such as defect migration, fission gas mobility, crack propagation, swelling, creep, and fuel-clad interactions. Instead, the project developed a metalorganic chemical vapor deposition system to grow epitaxial crystalline materials in any arbitrary crystalline orientation with extreme control over the thickness and orientation of the layers. In addition, the metalorganic chemical vapor deposition system allows for fine control of minor components for doping studies. This allows for interfacial/surface layers or uniform, intra-layer distributions of isotopes of interest to be incorporated into the grown crystalline or amorphous layers. In addition to the metalorganic chemical vapor deposition growth capabilities, bulk single crystal growth capabilities were developed. A uranium $(U)$ handling glove box and a radio frequency driven crystal furnace were commissioned to grow large diameter single crystal $U$ oxide samples from bulk feedstock. These capabilities will allow for the

\footnotetext{
${ }^{1}$ Idaho State University
} 
creation of large single crystals, bi-crystals, tri-crystals and the basis for materials with fixed void fractions, porosity, or alloyed materials. The large crystals have been cut and polished for diffusion, thermal conductivity, and grain restructuring studies at INL.

\section{Summary}

The LDRD developed the design for a unique, in situ irradiation capability ideally suited for the study of microstructure evolution of nuclear fuel materials. This capability can deliver prototypic reactor fluxes to study beginning of life fuel reorganization that drives the eventual fuel performance. The LDRD also achieved significant progress in advancing SEM technology for imaging microstructure phenomena in an irradiation environment. Prior to this investigation, it was unknown what impact the fission rate of nominal fuel during irradiation would have on the imaging quality of the SEM electron detection. Although additional study will be required to determine the impact with high free electron concentrations, there does not seem to be significant impact on the SEM resolution due to the fission fragments. The LDRD developed the capability to produce large diameter, single crystals of $U$ oxide that have been cut, polished, and used for thermal transport and grain boundary morphology studies at INL.

The LDRD successfully developed all three phases (irradiation, detection, and fabrication) of a separate effects testing capability. Advances in imaging and fabrication in the last 2 years, combined with the irradiation capability from the FY 2012 engineering design report, present an optimal path forward for realizing significant advances in the study of microstructural behavior of fuel material under realistic reactor conditions. This capability would bring a science-based understanding of the microstructural, time-dependent phenomena that drives the macroscopic fuel performance and would be a tremendous complement to the well-established irradiation cycle and post-irradiation examination experimental paradigm. A wealth of new information that informs advanced modeling and simulation is, at this time, a feasible and realistic goal for exponential advances in fuels and materials development.

\section{Benefits to DOE}

The work funded by this LDRD is in line with the larger strategy by DOE-NE to shift the paradigm of advanced nuclear fuels development to ensure the nation's energy security for the future. The historical approach of relying on experiential models and extensive qualification testing makes development of new fuels and advanced reactors a very time consuming and expensive process. Separate effects and isolated effects testing will inform new, first principle models to develop a predictive performance capability as fuels research moves forward. This project focuses on the three components (irradiation, fabrication, and detection) necessary for game-changing advances in nuclear fuels development, with the promise of shaving decades off the development time for new fuels needed in the next generation of U.S. nuclear power.

\section{Publications}

A draft journal article is being prepared for Nuclear Instruments and Methods describing the Cf-252 SEM imaging and measurements. A second article is being drafted to describe the accelerator-driven irradiation design and performance expectations. 


\title{
13-013 - Development of a Micromechanistic Phase-Field Modeling Approach for Life Estimation and Risk Assessment of Reactor Pressure Vessels
}

\author{
S. Bulent Biner, Michael R. Tonks, and Yongfeng Zhang
}

The integrity of reactor pressure vessels is one of the primary safety factors that limits the service life of current light water reactors. This LDRD aims at the development of a micromechanically based phase-field modeling algorithm in which co-evolution of microstructure due to radiation effects (i.e., precipitation of late blooming phases and hardening) and that due to damage evolution (i.e., change in the fracture morphology and shift in ductile brittle transition temperature curves) can be simulated in a unified framework for aging reactor pressure vessel steels.

\section{Summary}

The following is a brief summary of technical progress achieved during FY 2014:

The collective behavior of defect loops (self-interstitial atom and vacancy loops) on the segregation and precipitation behavior of allying elements copper $(\mathrm{Cu})$, manganese $(\mathrm{Mn})$, and nickel $(\mathrm{Ni})$ were studied parametrically, as shown in Figure 1. As can be seen, vacancy loops act as much stronger nucleation sites than the self-interstitial atom loops and also show the local stress state, resulting from the distribution of the loops, which dictates the evolution kinetics.
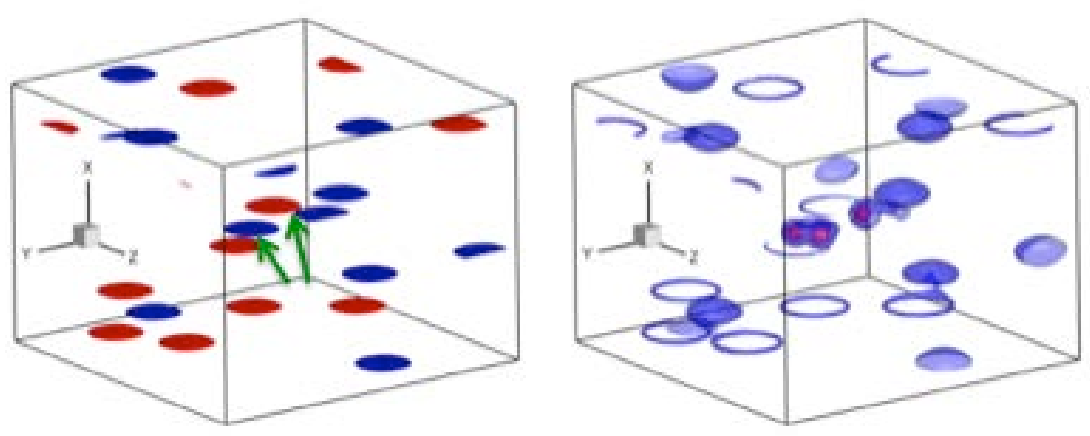

Figure 1. Concentration iso-surface for a randomly distributed equal number of vacancy and self-interstitial atom loops having loop sizes in the range of 5-10 Burger's vector (left figure) for $1 \mathrm{at} \% \mathrm{Cu}$, 1at\% Mn, and 1at\%Ni containing Fe-Cu-Ni-Mn alloy. After $t=8000$ simulation time, isosurfaces are for $5.0 a t \% C u$ (light blue) and 7.0at\% Cu (red) (right figure).

These results clearly demonstrate the role of underlying lattice defects and co-evolution. These findings will be partially presented at the 2014 Nuclear Materials Conference, and a full manuscript has been submitted to the journal of Acta Materialia.

Since dislocations are stronger sinks than the defect loops, our phase-field model for the large-scale evolution of dislocations has been modified to reach much higher dislocation densities then previously possible, as shown in Figure 2. 


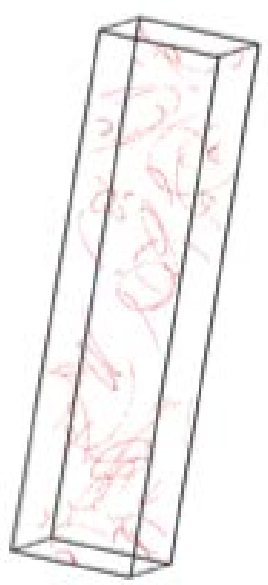

Strain $=0$

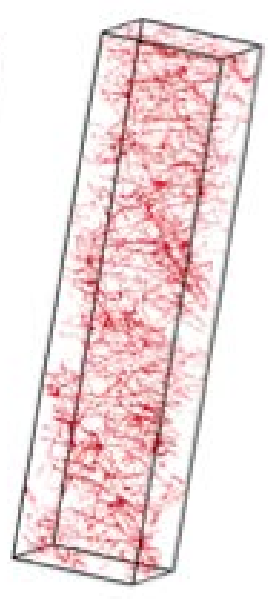

Strain $=5$

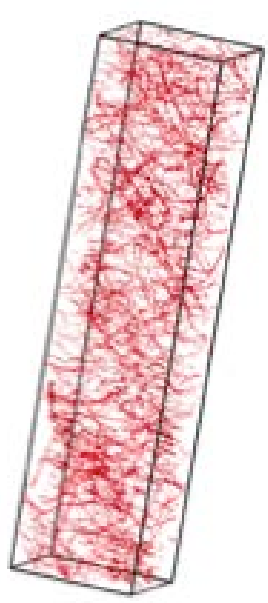

Strain $=15$

Figure 2. Evolution of dislocation density with $15 \%$ uniform strain in body-centered cubic iron (Fe) from initial dislocation density (right).

The precipitation simulations utilizing these dislocation substructures are currently being performed.

To represent the irradiation effects, the phase-field model has been modified to include the vacancy evolution as a function of the dpa (displacement per atom).

The phase-field code for fracture evolution has been parallelized for large-scale simulations.

The computational efficiency of the phase-field code to elucidate the interactions of dislocations with $\mathrm{Cu}$ precipitates, as shown in Figure 3, has improved, and the results obtained are in agreement with the molecular dynamics simulation; results are available in the literature.
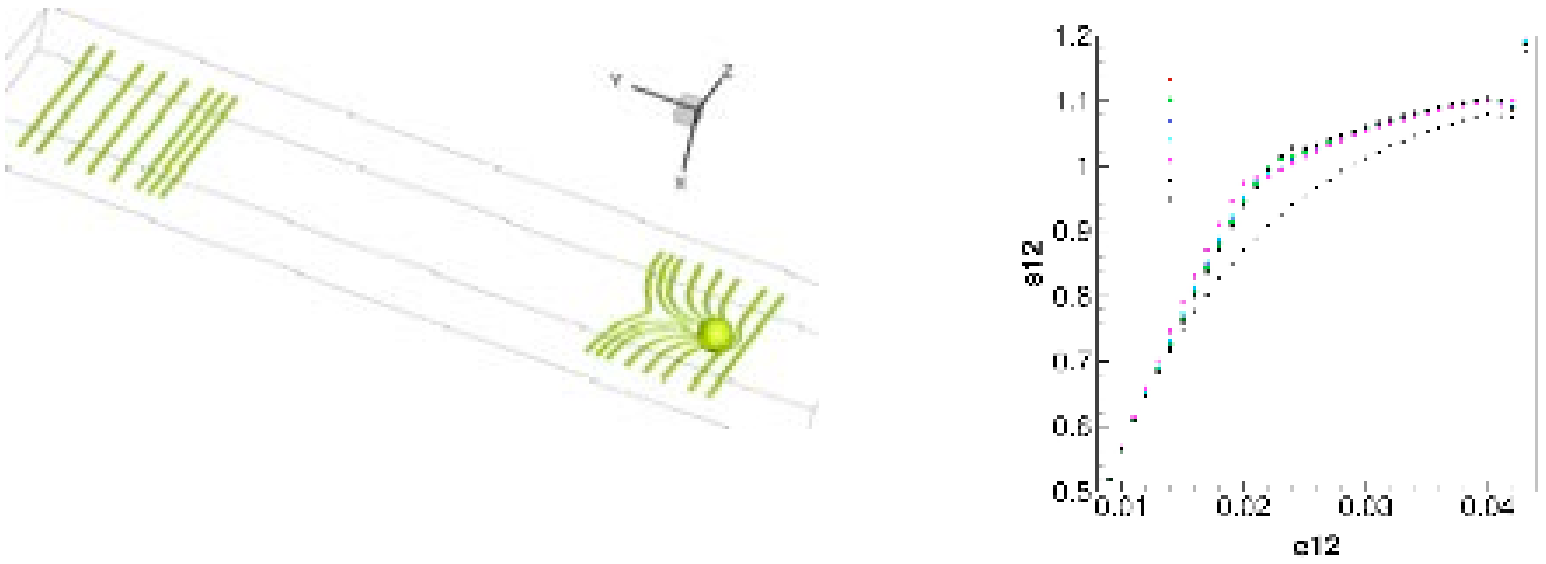

Figure 3. Phase-field simulation of interaction of an edge dislocation with a Cu precipitate in Fe and the resulting increase in the flow stress (hardening). 
As can be seen, we have achieved all planned FY 2014 project objectives. There are no changes in the scope or in the remaining yearly planned deliverables.

\section{Benefits to DOE}

For life assessment of current light water reactors to 60 years and beyond, it appears that the only viable alternative is to resort to the predictive modeling methodologies for the reactor pressure vessel steels embrittlement along with limited experiments. Our unified phase-field modeling approach aims at development of such a modeling algorithm.

\section{Publications}

Biner, S.B., W. Rao, and Y. Feng, "The Stability of Precipitates and the Role of Lattice Defects in Fe-1at\%Cu-1at\%Ni-1at\%Mn Alloy: A Phase-Field Model Study," Acta Mater., submitted, 2014.

\section{Presentations}

Rao, W., Y. Zhang, and S.B. Biner, "The Role of Lattice Defects on Segregation/Precipitation Behavior of a Dilute Fe-Cu-Mn-Ni Alloy," The Third International Workshop on Structural Materials for Innovative Nuclear Systems, Idaho Falls, ID, October 2013, submitted.

Biner, S.B., and Y. Zhang "A Phase-Field Model Study for Precipitation Behavior of a Dilute Fe-Cu-Mn-Ni Alloy," The Nuclear Materials Conference, Hilton Clearwater, FL, October 27-30, 2014. 


\title{
13-029 - In-Pile Detection of Crack Growth in the ATR
}

\author{
Joe Palmer, Sebastien Teysseyre, Joy Rempe, Gordon Kohse, and Steinar Solstad ${ }^{1}$
}

A key component in evaluating the ability of light water reactors to operate beyond 60 years is characterizing the degradation of materials exposed to radiation and various water chemistries. Of particular concern is the response of reactor materials to Irradiation Assisted Stress Corrosion Cracking. Some test reactors outside the United States, such as the Halden Boiling Water Reactor, have developed a technique to measure crack growth propagation during irradiation (see Figure 1). This technique incorporates a compact loading mechanism to stress the specimen during irradiation. A crack in the specimen is monitored using a reversing Direct Current Potential Drop (DCPD) method, which consists of introducing a precisely controlled current across a loaded specimen and measuring the drop in voltage across the crack mouth (which changes as a function of crack growth). This 3-year project applies the techniques used at the Halden Boiling Water Reactor to develop and evaluate a more robust test rig design that can survive the higher flux conditions at U.S. material testing reactors, such as INL's ATR and the Massachusetts Institute of Technology Research Reactor (MITR).

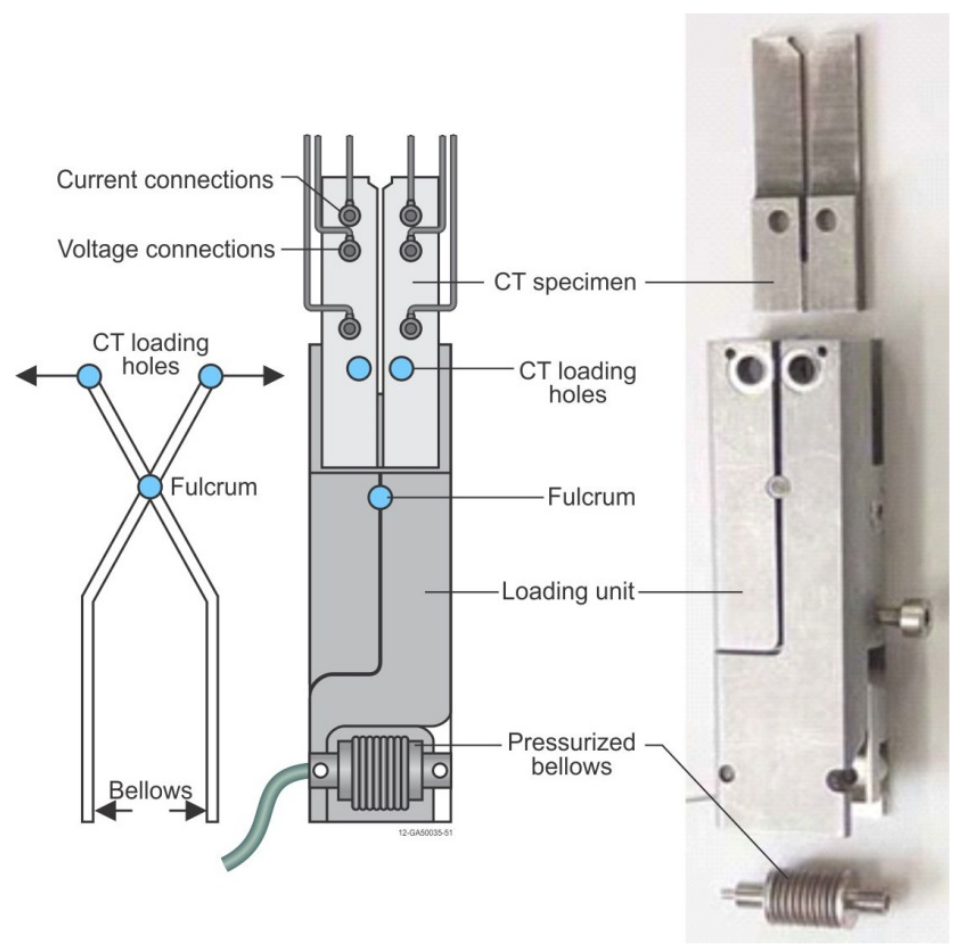

Figure 1. Schematic of a loading mechanism with DCPD connections.

\footnotetext{
${ }^{1}$ Halden Reactor Project
} 


\section{Summary}

The first year of this 3-year project was devoted to designing, analyzing, fabricating, and bench top testing a mechanism capable of applying a controlled stress to specimens while they are irradiated in a pressurized water loop (simulating pressurized water reactor conditions). Results from bench-top testing were very encouraging and showed that the load applied to the specimen could be predicted accurately for various pressures supplied to the bellows. During this past fiscal year (FY 2014), the second year of the project, the mechanism was tested in a high pressure, high temperature water environment outside of a reactor (i.e., in an autoclave). In addition, necessary documentation and safety reviews for testing in the MITR were completed. In the third year, the assembly will be tested in MITR and postirradiation examinations will be performed. After assessing results from this irradiation, ATR experiment engineers will also identify any design modifications required for adapting this technique for use in ATR Loop 2A.

Before the mechanism was tested in the autoclave, it was used to fatigue a specimen in air that had been instrumented with DCPD connections. The test proceeded very well for about 35 hours, producing a nice linear crack rate as shown in Figure 2. At the end of the 35-hour period, the specimen was removed from the loading mechanism and placed in a numerically controlled Instron machine. The control algorithm for the Instron machine was designed to match the calculated load applied by the loading mechanism (no direct measurement of load is possible with the loading mechanism). The crack rate obtained by the Instron machine (see Figure 3 ) was within $10 \%$ of that obtained using the loading mechanism, indicating that the loading mechanism was operating as predicted based on the magnitude of the fluctuating pressure applied to the bellows.

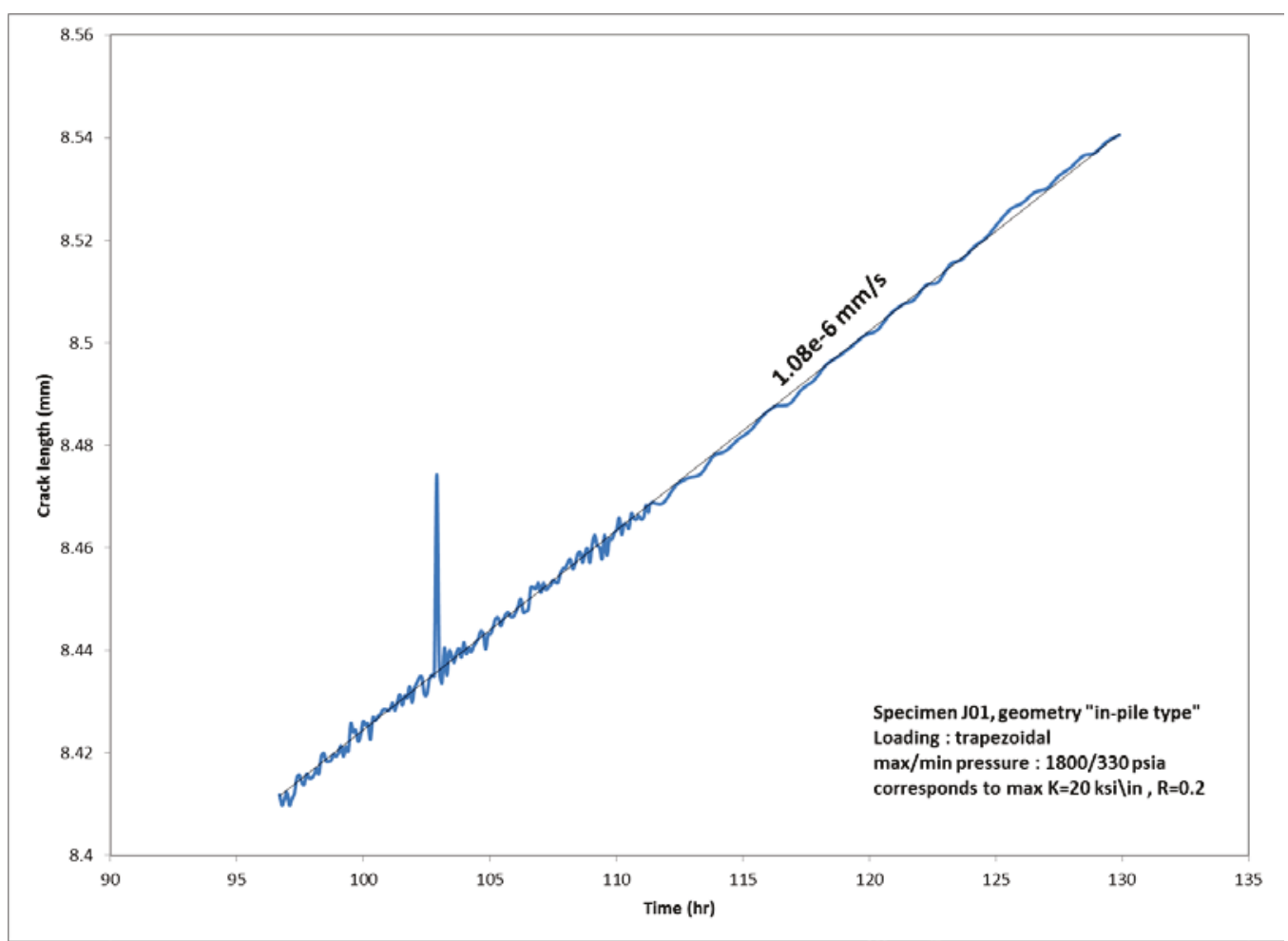

Figure 2. Crack length versus time obtained when fatiguing the specimen with a loading mechanism. 


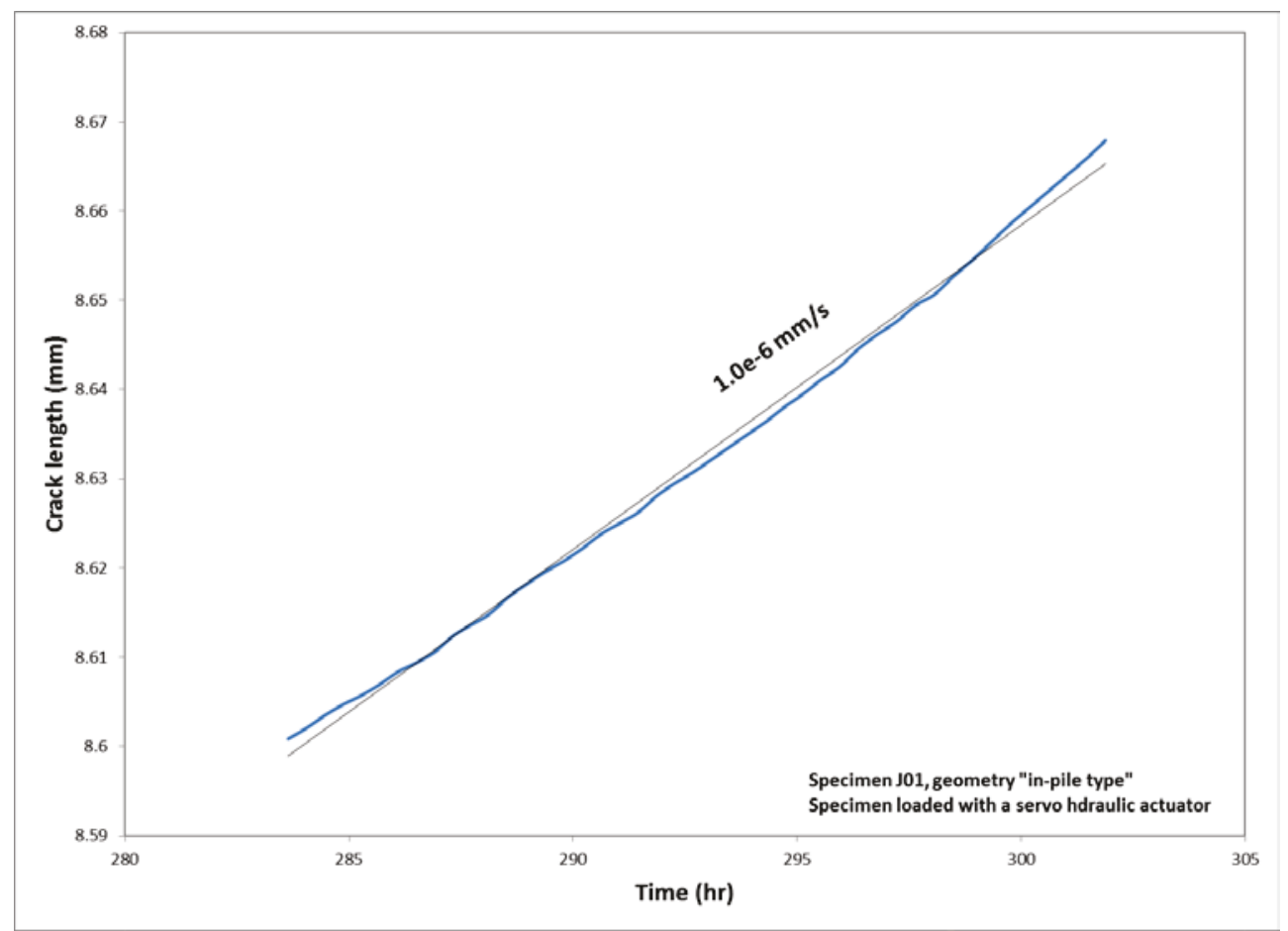

Figure 3. Crack rate obtained by Instron machine using loading profile calculated from a loading mechanism.

Figures 4 and 5 show the loader and grounding frame being assembled prior to being placed in the autoclave. The purpose of the alumina insulators is to keep the six leads from touching each other until they are grounded at the top of the grounding frame. This technique was copied from the work done at Halden and helps to ensure that stable signals can be passed out of the hot water environment to the DCPD data acquisition system.

The results of the autoclave testing were mixed. On the positive side, all the support equipment outside the autoclave worked well, including the pressure-regulating scheme, which cycled the pressure between high and low values every 25 seconds, and the DCPD signals were transmitted out of the autoclave and recorded properly by the digital acquisition system. This was a big plus because the major features of both of these systems will be used during the testing at MITR. On the negative side, the bellows attached to the loader developed a crack after about 10 hours of cycling. A different bellow has been procured and will be fatigue tested early in the third year of the project to verify this component will perform as required. 


\section{NUCLEAR SCIENCE \& TECHNOLOGY}

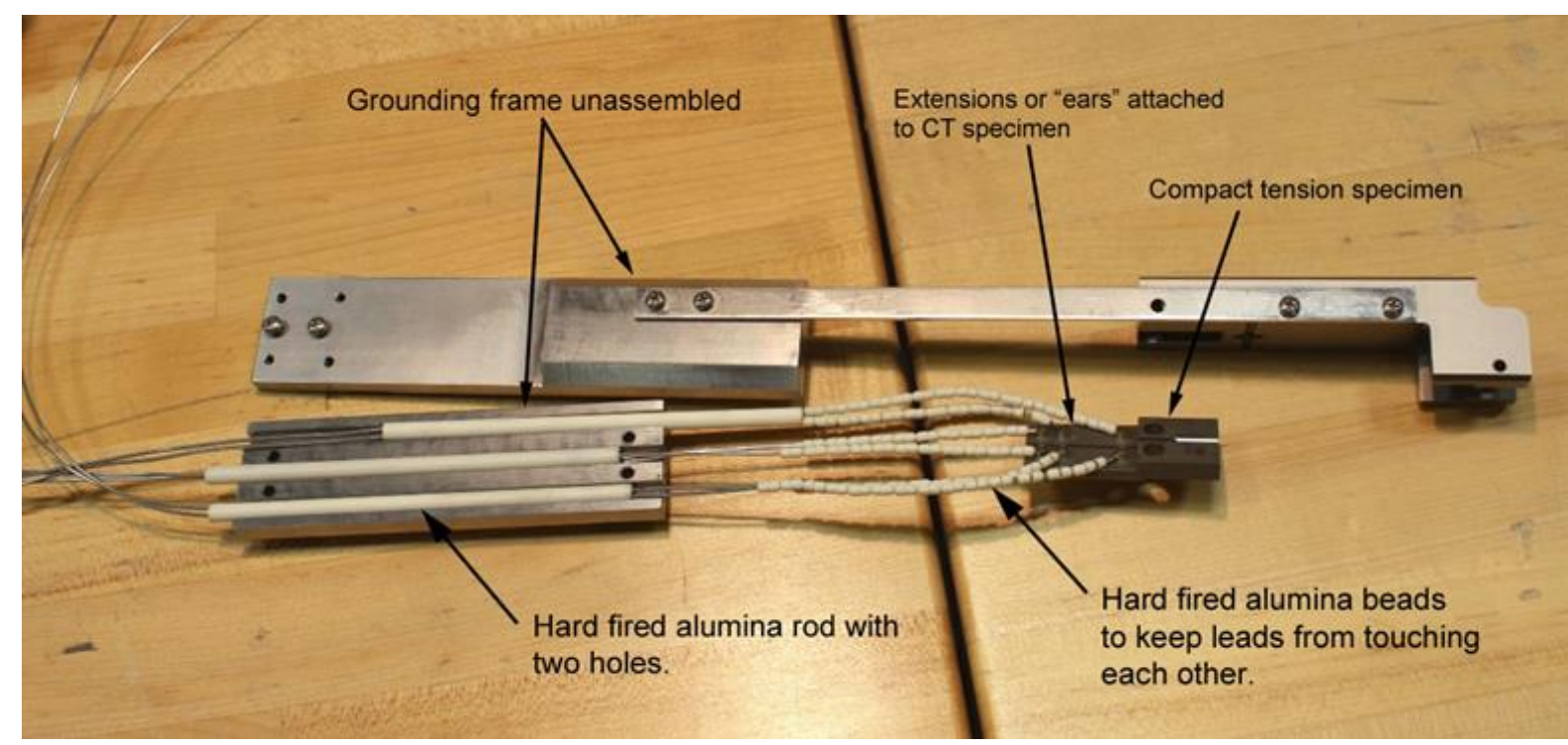

Figure 4. Specimen with leads attached prior to final assembly.

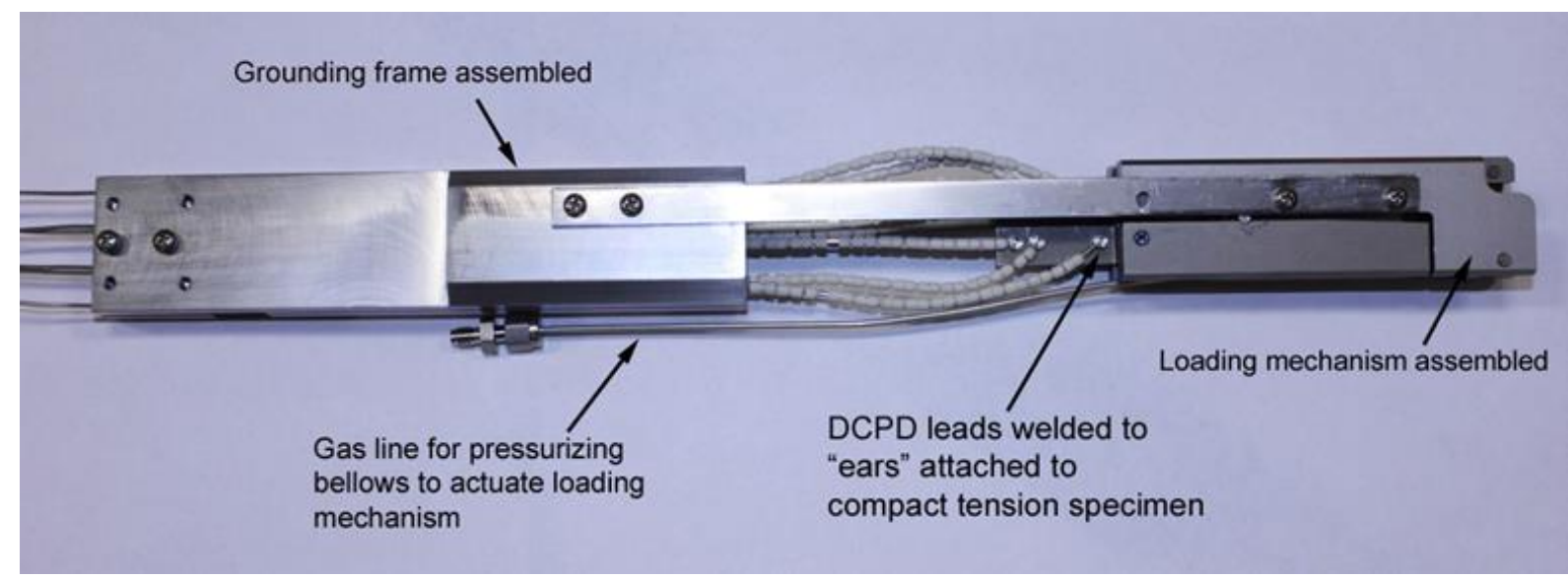

Figure 5. Completed assembly with loader strapped to grounding frame.

The FY 2014 work also included efforts to develop enhanced techniques for attaching leads to the specimens that will survive in a high pressure/high temperature water and radiation environment. Halden Reactor experience indicates that the electrical connections needed in the DCPD method must be extremely robust. Previously, INL designed and fabricated compact tension specimens with "ears" attached to the top (Figure 4). Electrical leads attached to the specimen ears consist of mineral insulated cable. The mineral insulated cable is laser welded to an intermediate boss, and the boss is resistance welded to the specimen ears (Figure 5). This process creates a very robust connection suitable for operation in the harsh in-core reactor environment.

While the loader was being refined and tested at INL, collaborators at the Massachusetts Institute of Technology were preparing hardware and safety analyses for the year three irradiation testing. The Massachusetts Institute of Technology group is on track to begin irradiation in the second quarter of 2015.

This research also helped fund an INL employee completing a University of Idaho graduate degree. 


\section{Benefits to DOE}

Development of advanced in-core instrumentation is critical to DOE's energy security mission to provide world-class facilities for advancing nuclear science and technology. Successful completion of this effort will result in a proven crack growth test rig that can be used by DOE-NE researchers and industry organizations to obtain real-time data for crack growth during irradiation testing in U.S. high flux material testing reactors.

\section{Publications}

Rempe, J., D. Knudson, J. Daw, T. Unruh, B. Chase, K. Davis, A. Palmer, and R. Schley, "Advanced In-pile Instrumentation for Materials Testing Reactors," IEEE T. Nucl. Sci., Vol. 61, 1984-1994, August 2014.

\section{Presentations}

Palmer, A., S. Teysseyre, K. Davis, J. Rempe, G. Kohse, Y. Ostrovsky, and D. Carpenter, "Adaptation of Crack Growth Detection Techniques to U.S. Material Test Reactors," International Congress on Advances in Nuclear Power Plants 2014 (ICAPP-14), Charlotte, NC, April 6-9, 2014.

Rempe, J., D. Knudson, J. Daw, T. Unruh, B. Chase, K. Davis, A. Palmer, and R. Schley, "Advanced In-pile Instrumentation for Materials Testing Reactors," 2013 Conference on Advancements in Nuclear Instrumentation, Measurements Methods (ANIMMA 2013), Marseilles, France, June 23-27, 2013. 


\title{
13-032 - Experimental and Computational Analysis of Hydride Microstructures in Zirconium in Dry Storage Conditions
}

\author{
Michael R. Tonks, Mark Carroll, Laura Carroll, Richard Williamson and Jason Hales
}

During reactor operation, oxidation of the Zircaloy cladding at the cladding/coolant interface results in hydrogen $(\mathrm{H})$ production. Some $\mathrm{H}$ is picked up by the cladding, and due to its low solubility in Zircaloy, it results in the formation of brittle hydrides. While these hydrides embrittle the cladding, they do not significantly impact the in-service performance of modern reactors. However, there is concern that hydrides may significantly impact the cladding integrity during long-term storage of used fuel.

One reason hydrides do not result in increased cladding failure during reactor operation is that the hydrides primarily form in a circumferential orientation around the cladding. After operation and removal from the reactor, the used fuel rods are stored in pools and then go through a drying process. During this process, the cladding heats up and some of the hydrides dissolve. As the temperature drops after drying, the hydrides can reform in a radial orientation due to higher pressure inside the rod. This radial orientation is much more problematic as it provides an easy crack path along the hydrides that can lead to through-wall cladding failure.

The purpose of this project is to develop a macroscale model that predicts the hydride orientation as a function of the stress and irradiation history. To accomplish this, we are using a coupled experimental and simulation approach in which a mesoscale model of hydride formation is developed and mesoscale experiments are conducted on unirradiated samples of hydrided zirconium $(\mathrm{Zr})$ and Zircaloy using INL facilities. This macroscale model will be used in INL's BISON fuel performance code to predict the impact of hydride reorientation on cladding integrity under used fuel dry storage conditions.

\section{Summary}

The project has been executed from the outset as two parallel efforts consisting of a modeling component and a supporting experimental component. In this summary, we will first explain the progress of the modeling and simulation development and then discuss the experimental efforts.

To facilitate accurate simulations, we have developed a model that predicts the formation and growth of the delta hydride phase in alpha $\mathrm{Zr}$, the most common phase found in used fuel cladding. To model the hydride phase nucleation and growth, we employ a phase field model using INL's mesoscale MARMOT code. As no models have yet been published for the delta hydride, we have developed a model specifically for that phase using thermodynamic free energies and experimentally measured lattice mismatches between the two phases. Preliminary results of this model are shown in Figure 1(a). In FY 2015 we will investigate the impact of applied stress on the hydride formation. 


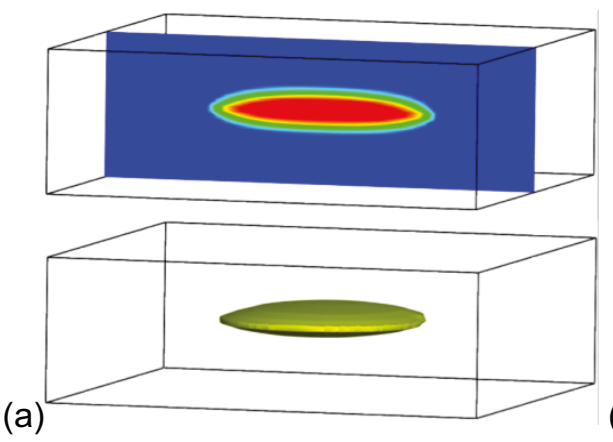

(b)

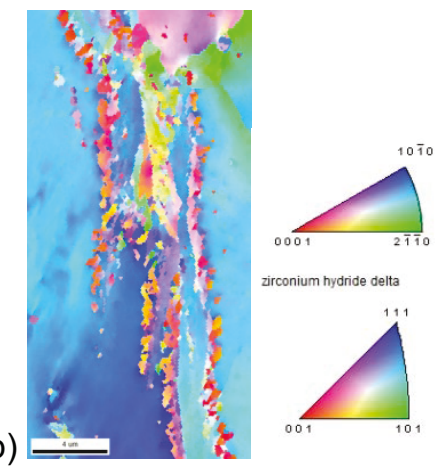

Figure 1. Examples of simulation and experimental work on hydride formation in Zr, where (a) is an example of a phase field simulation of 3-D hydride growth, and (b) shows an electron backscatter diffraction result from a hydrided sample.

To complement the modeling and simulation work, we have also performed laboratory-scale hydriding of $Z r-4$ and pure $\mathrm{Zr}$ samples and have utilized various approaches for characterizing the resultant microstructure. The first step of the experimental work was to develop a multi-step cold-rolling and annealing process to take beta-phase material obtained from a commercial alloy vendor and create alpha phase microstructures similar to those found in cladding. The second was to develop a process for hydriding the samples, with which we have obtained structures with various hydride concentrations.

The structure and orientations of the hydrides in the $\mathrm{Zr}$-based matrix are of particular interest, especially to facilitate comparison with the modeling and simulation results. Therefore, we have applied advanced microscopy techniques to hydrided $\mathrm{Zr}$ and $\mathrm{Zr}$-4 samples, including electron backscatter diffraction and transmission electron microscopy. These approaches are providing a detailed understanding of the hydride structure, the phase composition, and the impact of the hydride distribution on the surrounding material. See Figure $1(b)$ for an example of an electron backscatter diffraction image of a hydride. The refinement of these characterization techniques will be critical to the in and ex situ study of stress-based reorientation of Zr hydrides in FY 2015.

\section{Benefits to DOE}

This project will provide a material model necessary to predict the impact of hydride formation and reorientation on the cladding integrity under long-term dry storage conditions. This problem is currently of great interest to the DOE-NE Used Nuclear Fuel Disposition program, Electrical Power Research Institute, reactor vendors, and the major utilities.

\section{Publications}

Carroll, M., L. Carroll, B. Fromm, W. Swank, D. Haggard, and M. Tonks, "Nucleation and Growth of Hydride Precipitates Chains in Zr and Zr-4," Met. Trans. A, in preparation.

\section{Presentations}

Carroll, M., L. Carroll, B. Fromm, W. Swank, D. Haggard, and M. Tonks, "Characterization of Delta Hydride Precipitates in Pure Zr and Zr-4," TMS Annual Meeting, Orlando, FL, March 15-19, 2015. 


\title{
13-039 - Induction Based Fluidics Mass Spectrometry for Characterizing Radioactive Extraction Solvents
}

\author{
Gary S. Groenewold, Chris A. Zarzana, Dean Peterman, and Bruce J. Mincher
}

Over the past several years, electrospray ionization (ESI) mass spectrometry (MS) has been invaluable for characterizing the effects of radiation on solvent extraction systems used in fuel cycle separations research. ESI-MS functions by spraying droplets at a sampling aperture, transferring charged solutes into the gas phase where they can be analyzed by MS. In conventional ESI-MS, the majority of the sample solution is deposited on the outside of the aperture. Usually this is inconsequential, but if the sample contains significant levels of radioactive contamination, radiological control requirements preclude the use of ESI-MS.

Current research objectives are to minimize the volume of sample used and to improve the transfer efficiency into the mass spectrometer, without compromising analytical quality. In the first year of the project, new technology referred to as induction-based fluidics was used to deliver nanoliter-sized droplets directly into the ESI aperture. Mass spectra of carbamoylmethylphosphine oxide derivatives and associated lanthanide coordination complexes were acquired, which showed that for this type of sample, volumes as small as 20 nanoliters could produce a high quality mass spectrum. These results suggested that the technology could function within radiological control requirements at the Central Facilities Area; thus, efforts during FY 2014 emphasized modification of an instrument for a radiological environment.

\section{Summary}

A bench-top mass spectrometer was prepared for operation within radiological buffer areas at INL; the instrument was subjected to extensive maintenance and thoroughly benchmarked using non-radioactive samples. Since the instrument operates on a low-frequency duty cycle, droplet-to-droplet variations were eliminated by implementing low-flow electrospray. A capillary nanospray needle (Figure 1, left) operated at 50 nanoliters/min produced excellent mass spectra in a few seconds, indicating that a total sample volume on the order of a few nanoliters is all that is required.

Furthermore, it is likely that analysis of even lower volumes can be achieved using a syringe pump capable of lower flow rate regimes.

As part of the performance testing of the low flow, low volume ESI mass spectrometer, a series of lanthanide-nitrate
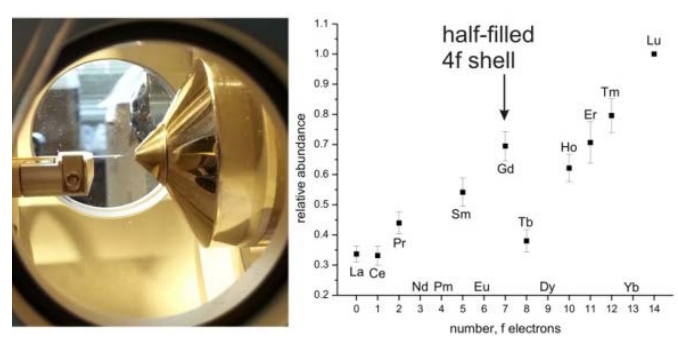

Figure 1. Left, photograph of the capillary nanospray needle and sampling cone. Right, plot of $\left[\mathrm{Ln}\left(\mathrm{NO}_{3}\right)_{4}\right]$ abundances relative to $\left[\mathrm{Lu}\left(\mathrm{NO}_{3}\right)_{4} J\right.$, versus number of $\mathrm{f}$-electrons in the valence shell, showing the discontinuity corresponding to the half-filled $4 f$ shell. Neodymium (Nd), promethium (Pm), europium (Eu), dysprosium (Dy) and ytterbium $(\mathrm{Yb})$ were not tested. competition experiments were performed in which solutions having equimolar concentrations of lanthanide elements ranging from lanthanum (La) to lutetium (Lu) were analyzed as nitrate solutions. As the atomic number of the lanthanides increases, their ionic radius decreases in a regular fashion, producing a progressively harder cation, with stronger ionic bonding to nitrate (Figure 1, right). However, a surprising discontinuity is seen at $\mathrm{Gd}^{3+}$, which has a $4 f^{7}$ occupancy that correlates with a half-filled 
$4 f$ shell. The half-filled shell is contracted relative to the next element, $\mathrm{Tb}^{3+}$, which has an electron configuration of $4 f^{8}$, disproportionately expanding the $4 f$ shell and resulting in a softer cation and a lower abundance nitrato complex. The result demonstrates the remarkable sensitivity to subtle variations in $f$-block chemistry.

Another objective is to replicate the chromatography using a low sample volume, low flow approach. Liquid chromatograph operations would be complicated in a radiological environment. An electrospray capillary needle packed with a chromatographic stationary phase can likely provide separation without any modification to the electrospray experiment. A combined capillary column-electrospray needle was specified and procured for separations experiments. Injection of $39 \times 10^{-12} \mathrm{~g}$ (39 picograms) of carbamoylmethylphosphine oxide showed a defined elution peak (Figure 2), which is expected to further resolve using the packed needle.

\section{Benefits to DOE}

There is an ongoing need for the capability to chemically measure

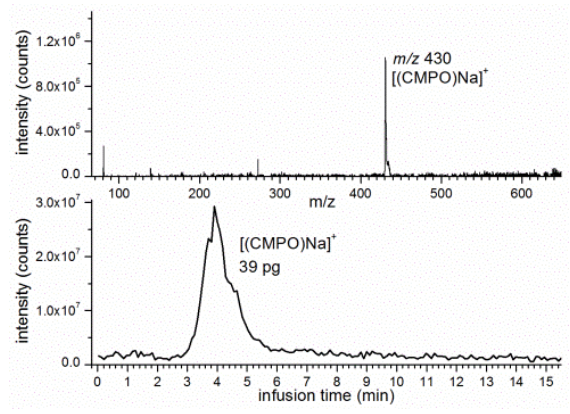

Figure 2. Top, mass spectrum, 39 picograms of carbamoylmethylphosphine oxide. Bottom, ion profile, $\mathrm{m} / \mathrm{z} 430$. vanishingly small sample volumes that exist in each of the three INL mission areas: nuclear energy, national security, and sustainable energy. The need is especially acute for scenarios in which the sample size is small or poses risks associated with toxicity or radioactivity. ESI-MS provides excellent information on the solution solutes, but is not compatible with radioactive samples because the majority of the sample is deposited on the ESI source where it constitutes loose contamination. The induction-based fluidics project produced an ESI-MS instrument that will provide detailed molecular information for fuel cycle separations without contaminating the exterior surfaces. The instrumentation will constitute a capability that is rare in the analytical chemistry and radiochemistry communities and will significantly enhance INL's competitive position in the fuel cycle separations area and in process control, since the proposed technology is capable of solution analysis using exceedingly small volumes.

\section{Publications}

Groenewold, G., A. Sauter Jr., and A. Sauter III, "Rapid Analysis of Single Droplets of Lanthanide-Ligand Solutions by Electrospray lonization Mass Spectrometry using an Induction Based Fluidics Source," Anal. Chem. Vol. 85, 6398-6404, 2013. 


\title{
13-040 - Epistemic Uncertainty Quantification in Dynamic Probabilistic Risk Assessment
}

\author{
Joseph Nielsen, Akira Tokuhiro, ${ }^{1}$ and Robert Hiromoto ${ }^{1}$
}

Dynamic Probabilistic Risk Assessment is a powerful tool that provides a more rigorous analysis of complex dynamic systems than classical risk assessment tools such as fault trees/event trees. Dynamic Probabilistic Risk Assessment methods allow for coupling to simulation tools such as RELAP5, which captures the actual timing of events rather than utilizing an expert knowledge based approach that results in grouping multiple transients together. Uncertainty quantification, both aleatory and epistemic, can be evaluated concurrently within the simulation. Aleatory uncertainty refers to random or stochastic uncertainty, which is inherent to the system under study. Epistemic uncertainty refers to the uncertainty of the state of limited knowledge about the system on the part of the analyst. Epistemic uncertainty can be narrowed or eliminated with additional knowledge.

Dynamic Probabilistic Risk Assessment methods have a disadvantage as the result of combinatorial or state explosion - as the number of potential system states grows, the size of the simulation grows exponentially. Solution methods utilize either a Monte Carlo based approach or Discrete Dynamic Event Trees (DDET). This research has focused on the use of DDET with the use of the branch-and-bound algorithm and dynamic programming methods to solve the DDET efficiently and limit the potential for state explosion. This allows us to focus on scenarios and timing of events that lead to failure and discard or prune branches of the DDET that yield a success (i.e., cold safe shutdown) or low probability of failure branches. In many cases, with regard to failure of equipment or systems, failure rates are extremely small (approximately $1 \times 10^{-6} / \mathrm{hr}$ ). The algorithm can direct the simulation to high probability failure scenarios and conditions for efficient uncertainty quantification for both epistemic and aleatory uncertainty. Further, epistemic uncertainty can be quantified and ranked where additional information regarding modeling fidelity and validation experiments can yield the greatest amount of risk reduction.

\section{Summary}

During the 2 years of this project, implementation of the branch-and-bound algorithm was demonstrated and incorporated into the RAVEN framework. The branch-and-bound algorithm is an optimization algorithm intended to find the maximum (or minimum) value of a function associated with combinatorial explosion problems. In collaboration with the University of Idaho, we have co-developed and integrated a set of tools in a consistent manner, in particular, a potentially practical coupling of thermal-hydraulic codes (e.g., RELAP5-3D), a "controller tool" to start and stop the time-dependent calculation, definition of sets per translation of expert/experiential knowledge of reactor transients, dynamic programming as means to establish linked conditions and constraints on event branches, and a branch-and-bound (programming) approach to "trim" branches yielding desirable outcomes (such as cold shutdown condition) or when the interest is the opposite, those events leading to potential core meltdown. Furthermore, we have adopted the LENDIT metrics to essentially reduce the system characterization in a consistent manner. LENDIT metrics, which represent length, energy, number, distribution, information, and time, can be used with State, System, Response, Resource (S2R2) sets. Linking of these elements is facilitated by utilization of expert/experiential knowledge, referred to as "knowledge base mining." This allowed us to establish constraints and bounding functional relationships to support the branch-and-bound algorithm. The use of LENDIT metrics for the reduction of system characterization has been evaluated to be consistent with the operator splitting techniques employed in the RAVEN framework.

\footnotetext{
${ }^{1}$ University of Idaho
} 
The evaluation of DDET was expanded to evaluate epistemic modeling uncertainty in the thermal-hydraulic models. Demonstration cases were developed for a Pressurized Water Reactor (PWR) Station Blackout (SBO) transient utilizing the Westinghouse 4-Loop PWR reference plant and a General Electric Boiling Water Reactor (BWR) SBO. Computational time associated with the PWR SBO was demonstrated to be reduced by $75 \%$, allowing for greater resources to be focused on sensitivity analysis associated with epistemic uncertainty in physical modeling. The BWR SBO incorporated a greater degree of complexity as it evaluated conditions such as safety relief valves failing to close, reactor coolant pump seal loss of coolant accidents, and the timing of events associated with the loss of alternating current power and battery backed power. The reduction in computing time associated with the BWR SBO transient was determined to be approximately $66 \%$. A computational efficiency of $60 \%$ for a DDET framework can be realized as implemented.

In collaboration with students from the University of Idaho, the sensitivity analysis was expanded to develop a Phenomenological Identification and Ranking Table (PIRT) for both the PWR and BWR transients. The PIRT process utilized a Latin Hypercube Sampling algorithm combined with the Spearman Rank Correlation matrix. The correlation matrix provides values that reflect the degree of correlation between sampled values and response functions. Applying this methodology to thermal-hydraulics codes allows for ranking parameters based on the highest degree of correlation. A summary of the PIRT correlation matrix for the PWR and BWR SBO cases for a select set of parameters defined from the LENDIT metrics described above are presented in Table 1 and Table 2. Table 1 provides a summary of the PWR SBO analysis with various combinations of emergency cooling from the centrifugal charging pumps (CCP) and the safety injection pumps (SI). A summary of the BWR SBO transient for a scenario involving firewater recovery is provided in Table 2. Modeling improvements and validation associated with those models can be defined.

\section{Table 1. PIRT summary for PWR SBO transient.}

\begin{tabular}{|c|c|c|c|c|c|c|c|c|}
\hline \multirow[b]{2}{*}{ Parameter } & \multicolumn{8}{|c|}{ Emergency Cooling Pump Availability } \\
\hline & $\begin{array}{c}2 \mathrm{CCP} \\
2 \mathrm{SI}\end{array}$ & $\begin{array}{c}2 \mathrm{CCP} \\
1 \mathrm{SI}\end{array}$ & $\begin{array}{c}2 \mathrm{CCP} \\
0 \mathrm{SI}\end{array}$ & $\begin{array}{c}1 \mathrm{CCP} \\
2 \mathrm{SI} \\
\end{array}$ & $\begin{array}{c}1 \mathrm{CCP} \\
1 \mathrm{SI}\end{array}$ & $\begin{array}{c}1 \mathrm{CCP} \\
0 \mathrm{SI}\end{array}$ & $\begin{array}{c}0 \mathrm{CCP} \\
2 \mathrm{SI} \\
\end{array}$ & $\begin{array}{c}0 \mathrm{CCP} \\
1 \mathrm{SI}\end{array}$ \\
\hline Initial Power & $\begin{array}{c}0.025 \\
\text { Low }\end{array}$ & $\begin{array}{c}0.079 \\
\text { Low }\end{array}$ & $\begin{array}{c}0.144 \\
\text { Low }\end{array}$ & $\begin{array}{c}0.291 \\
\text { Low }\end{array}$ & $\begin{array}{c}0.041 \\
\text { Low }\end{array}$ & $\begin{array}{c}0.024 \\
\text { Low }\end{array}$ & $\begin{array}{c}0.088 \\
\text { Low }\end{array}$ & $\begin{array}{c}0.149 \\
\text { Low }\end{array}$ \\
\hline Safety Rod Worth & $\begin{array}{c}0.035 \\
\text { Low } \\
\end{array}$ & $\begin{array}{c}0.108 \\
\text { Low } \\
\end{array}$ & $\begin{array}{c}0.054 \\
\text { Low }\end{array}$ & $\begin{array}{c}0.011 \\
\text { Low }\end{array}$ & $\begin{array}{c}0.159 \\
\text { Low }\end{array}$ & $\begin{array}{c}0.024 \\
\text { Low }\end{array}$ & $\begin{array}{c}0.064 \\
\text { Low } \\
\end{array}$ & $\begin{array}{c}0.038 \\
\text { Low }\end{array}$ \\
\hline PORV & $\begin{array}{c}0.161 \\
\text { Low }\end{array}$ & $\begin{array}{c}0.212 \\
\text { Low }\end{array}$ & $\begin{array}{c}0.018 \\
\text { Low }\end{array}$ & $\begin{array}{c}0.544 \\
\text { Med }\end{array}$ & $\begin{array}{c}0.203 \\
\text { Low }\end{array}$ & $\begin{array}{c}0.666 \\
\text { Med }\end{array}$ & $\begin{array}{c}0.625 \\
\text { Med }\end{array}$ & $\begin{array}{c}0.805 \\
\text { High }\end{array}$ \\
\hline $\mathrm{CCP}$ & $\begin{array}{l}0.328 \\
\text { Med }\end{array}$ & $\begin{array}{c}0.313 \\
\text { Med }\end{array}$ & $\begin{array}{c}0.548 \\
\text { Med }\end{array}$ & $\begin{array}{c}0.460 \\
\text { Low }\end{array}$ & $\begin{array}{c}0.498 \\
\text { Med }\end{array}$ & $\begin{array}{c}0.780 \\
\text { High }\end{array}$ & $\mathrm{N} / \mathrm{A}$ & $\mathrm{N} / \mathrm{A}$ \\
\hline SI & $\begin{array}{c}0.129 \\
\text { Low }\end{array}$ & $\begin{array}{c}0.178 \\
\text { Low }\end{array}$ & $\mathrm{N} / \mathrm{A}$ & $\begin{array}{c}0.244 \\
\text { Low }\end{array}$ & $\begin{array}{c}0.179 \\
\text { Low }\end{array}$ & N/A & $\begin{array}{c}0.190 \\
\text { Low }\end{array}$ & $\begin{array}{c}0.122 \\
\text { Low }\end{array}$ \\
\hline
\end{tabular}




\section{NUCLEAR SCIENCE \& TECHNOLOGY}

Table 2. PIRT for BWR SBO transient with firewater recovery.

\begin{tabular}{|c|c|c|}
\hline Parameter & $\begin{array}{c}\text { Correlation Coefficient } \\
\text { (PCT) }\end{array}$ & PIRT Ranking \\
\hline Safety Release Valves flow area & -0.194 & Low \\
\hline HPCl capacity & 0.051 & Low \\
\hline RCIC capacity & 0.203 & High \\
\hline Firewater capacity & -0.930 & High \\
\hline Power & 0.817 & Low \\
\hline Zr thermal conductivity & 0.063 & Low \\
\hline Zr heat capacity & -0.026 & \\
\hline
\end{tabular}

\section{Benefits to DOE}

The methods and algorithms developed during this research can have a significant benefit to DOE's nuclear energy mission. Improving the capability to quantify risk with regard to modeling and simulation can result in significant cost saving. Validation experiments can be efficiently designed to provide data involving risk reduction. These algorithms have been implemented using object-oriented methodology within the RAVEN framework, which is under development as part of the MOOSE project. The algorithms and methodology developed in this research can be extended beyond the core damage analysis for nuclear power plants. Economic risk evaluations associated with plant upgrades and modifications may be incorporated. These applications can be used to support risk analysis in chemical and aerospace industries as well.

\section{Publications}

Nielsen, J., A. Tokuhiro, R. Hiromoto, and L. Tu, "Branch-and-Bound Algorithm Applied to Uncertainty Quantification of a Boiling Water Reactor Station Blackout," Nuclear Engineering and Design, submitted.

Nielsen, J., A. Tokuhiro, R. Hiromoto, and J. Khatry, "Method to Branch-and-Bound Large SBO State Spaces Under Dynamic Probabilistic Risk Assessment via Use of LENDIT Scales and S2R2 Sets," Journal of Nuclear Science and Technology, Vol. 51, 1212-1230, 2014.

\section{Presentations}

Nielsen, J., A. Tokuhiro, and R. Hiromoto, "Method to Branch and Bound Large SBO State Spaces under Dynamic Probabilistic Risk Assessment via Use of LENDIT Scales and S2R2 Sets," Probabilistic Safety Assessment and Management Conference, Tokyo, Japan, April 15-18, 2013.

Nielsen, J., A. Tokuhiro, and R. Hiromoto, "Branch-and-Bound Algorithm Applied to Discrete Dynamic Event Trees," Transactions of the American Nuclear Society, 2013 Winter Meeting, Washington, DC, November 2013.

Nielsen, J., A. Tokuhiro, and R. Hiromoto, "Sensitivity and Uncertainty Quantification Analysis within a Dynamic Event Tree Framework," Transactions of the American Nuclear Society, 2014 Summer Meeting, Reno, NV, June 2014. Nielsen, J., Branch-and-Bound Algorithm Applied to Dynamic Event Trees and Uncertainty Quantification in Nuclear Reactors, Doctoral Dissertation, December 2014. 


\title{
13-050 - Concurrent Atomistic to Macroscale Modeling of Materials under Irradiation Using the Phase Field Crystal Model
}

\author{
Yongfeng Zhang, S. Bulent Biner, Richard Williamson, Derek Gaston, Michael Tonks, Katsuyo Thornton, ${ }^{1}$
} and Dmitry Karpeev ${ }^{2}$

Radiation induces change in microstructure through production, transport, and accumulation of lattice defects which alter the material properties. This phenomenon spans multiple time (from picoseconds to years) and length (from angstroms to meters) scales and raises challenges for predictive modeling. This project seeks to develop a multiscale model that bridges the atomistic to the macroscale for radiation damage in materials by capturing the critical mechanisms occurring during the early stages of irradiation. To accomplish this goal, the phase field crystal (PFC) model is being implemented into INL's MARMOT code, which is based on the MOOSE framework. The PFC model features atomic resolution and a diffusive time scale and represents the first use of MOOSE at the atomistic scale. By coupling with MOOSE-BISON-MARMOT, it is expected to be capable of modeling radiation damage in materials over multiple time and length scales.

The main scope of this project has come to include: (1) developing a new, PFC, free energy functional specific for body-centered cubic (bcc) metal capable of describing point defects such as vacancies, (2) implementing the new model into MARMOT, and (3) coupling with BISON by passing material properties such as elastic constants.

Accomplishing these goals will provide an atomistic to engineering scale capability within the MOOSE-BISON-MARMOT framework.

\section{Summary}

This project is currently at the end of its second fiscal year. The main tasks of FY 2014 were to (1) implement the rational-function-fit (RFF) free energy functional newly developed by our collaborators at University of Michigan into MARMOT, (2) start elastic constant calculation using MARMOT, and (3) develop a PFC model capable of describing vacancies.

Within the first 2 years, we have successfully implemented two PFC models into MARMOT and solved them using the MOOSE framework. The polynomial PFC model from "Thermodynamics of BCC Metals in Phase-Field-Crystal Models" by Jaatinen et al. was implemented in FY 2013, and the RFF PFC model from "Classical Density Functional Theory and the Phase-Field Crystal Method Using a Rational Function to Describe the Two-Body Direct Correlation Function" by

Pisutha-Arnond et al. in FY 2014. As shown in Figure 1,

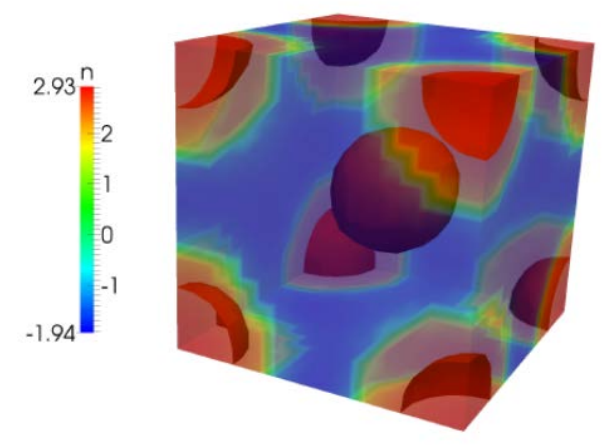

Figure 1. Stabilized bcc Fe lattice in a MARMOT simulation using the RFF model. The atoms can be seen by the density ( $n$ ) contour.

\footnotetext{
${ }^{1}$ University of Michigan

${ }^{2}$ Argonne National Laboratory
} 
the solidification process of a 3-D bcc iron (Fe) lattice has been simulated using MARMOT with the RFF model. To solve the RFF model in MOOSE, a multi-grid preconditioner has been developed with help from Dmitry Karpeev at Argonne National Laboratory to efficiently solve the Helmholtz problems using the finite-element method.

The calculation of elastic properties in PFC has been attempted in literature. However, the results obtained are in question due to the difficulty to conserve the total mass while applying deformation. Previously, our collaborator at University of Michigan has proposed a way to calculate the 11 and 12 type elastic moduli with conserved mass. In FY 2014, a new approach was proposed to evaluate the deformation energy based on the undeformed configuration and the deformation matrix so that the shear type (44) elastic moduli can be calculated as well. In Figure 2, this new approach was demonstrated using the one-mode approximation for bcc crystal. As shown in Figure 2(a), the deformation energy curve exhibits a parabolic shape with shear strain, and a dependence on the mesh size is noticed. The elastic moduli (i.e., the second derivative of deformation energy) can be accurately calculated with sufficient spatial resolution, as shown in Figure 2(b).

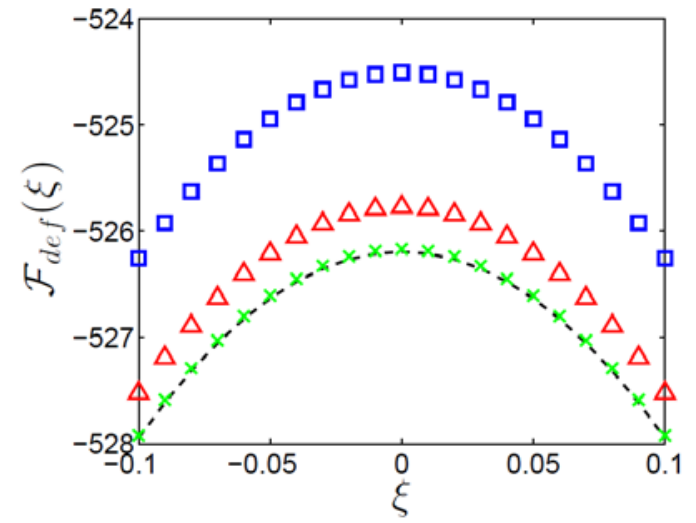

(a)

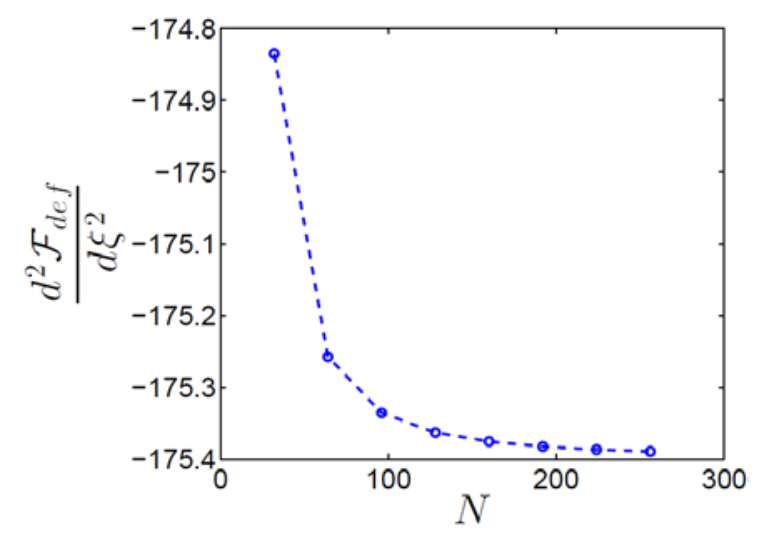

(b)

Figure 2. (a) Deformation energy versus shear strain $\xi$ with the mesh size being 0.278 (square; $N=32$ ), 0.139 (triangle; $N=64$ ), and 0.035 (cross; $N=256$ ) in unit of lattice parameter, and $(b)$ its second derivative versus $N$, total number of mesh grids.

In FY 2014, development of a PFC model for vacancies started at the University of Michigan. By adding a penalty term in the free energy when the local mass density goes negative, vacancies can be stabilized within a window specified by the under cooling parameter $(r)$ and the average mass density $\left(\phi_{0}\right)$. In addition, it was found that for each combination of $\left(r, \phi_{0}\right)$, there exists an equilibrium number of vacancies denoted by a minima in total free energy.

FY 2015 tasks will be focused on radiation damage and coupling with BISON. For this purpose, the PFC model for vacancies will be implemented into MARMOT. The elastic constants with and without vacancies will be calculated. The results will be passed into BISON for an engineering scale evaluation of radiation-induced change in elastic property. 


\section{Benefits to DOE}

This project will benefit the DOE missions of energy resources and science by developing a new multiscale capability for radiation damage in materials. Such a capability provides an opportunity for concurrent modeling of radiation damage at the microstructure level and the resulting change in material properties at the engineering level. The accomplishment will improve the predictive modeling on radiation damage and thus help the design of advanced fuels and nuclear materials.

\section{Publications}

Chan, V., N. Pisutha-Arnond, and K. Thornton, "Numerical Implementation of Deformation in the Phase-Field Crystal Model," in preparation.

Ghosh, S., and K. Thornton, "An Efficient Real-Space Method for Classical Density Functional Theory (cDFT) and Phase-Field Crystal Models in Two-Dimensions," in preparation.

Montiel, D., and K. Thornton, "Stability Analysis of Individual Vacancies Using the Vacancy Phase-Field Crystal Model," in preparation.

\section{Presentations}

Montiel, D., and K. Thornton, "Analysis of the Stability and Diffusion of Individual Vacancies Using the Vacancy Phase-Field Crystal Model," TMS 2015: 144th Annual Meeting and Exhibition, Orlando, FL, USA, March 2015, accepted.

Chan, V., N. Pisutha-Arnond, and K. Thornton, "Calculations of Isothermal Elastic Constants in a Classical DFT-Based Phase-Field Crystal Model," U.S. National Congress on Theoretical and Applied Mechanics 2014, Lansing, MI, June 15-20, 2014. (Invited)

Ghosh, S., V. Chan, and K. Thornton, "A Novel Approach to Obtain Approximate Analytical Direct Correlation Function for use in Classical Density Functional Theory," U.S. National Congress on Theoretical and Applied Mechanics 2014, Lansing, MI, June 15-20, 2014.

Butterfield, A., V. Chan, Y. Zhang, M. Tonks, K. Thorton, and D. Karpeev, "Obtaining Elastic Constants using Phase Field Crystal Modeling," ANS Annual Meeting, Reno, NV, June 15-19, 2014.

Chan, V., N. Pisutha-Arnond, K. Elder, and K. Thornton, "Phase-Field Crystal Model and Thermodynamics of Elastically Stressed Solids," Materials Science \& Technology 2013 Meeting, Montreal, Canada, October 29, 2013. 


\title{
13-071 — Advanced Fracture Modeling for Nuclear Fuel
}

\author{
Benjamin Spencer, Hai Huang, Jason Hales, Derek Gaston, and John Dolbow ${ }^{1}$
}

Fracture in ceramic nuclear fuel plays an important role in its thermal and mechanical behavior. Currently employed techniques for fracture modeling in fuel have well-known mesh dependencies. Improved fracture modeling for nuclear fuel is essential for predictive modeling of fuel behavior in conditions such as accidents, which are outside the range of applicability of current fuel behavior models. This project applies two fracture modeling techniques to fuel performance modeling: the extended finite element method (XFEM) and the discrete element method (DEM). Both of these techniques are well known to overcome the weaknesses of methods used in current practice but have had limited applications to multiphysics problems and, to our knowledge, have not been applied to nuclear fuel modeling. This project is making these methods available in the BISON nuclear fuel performance modeling code and demonstrating their applicability to nuclear fuel simulation. This will strengthen our ability to predict fuel behavior in normal and accident conditions.

\section{Summary}

This project, which just completed its second year, consists of concurrent efforts to apply the XFEM and DEM fracture modeling techniques to nuclear fuel. In XFEM, the standard finite element method is enhanced to permit it to model discontinuities in solution fields, such as those that occur across a crack. The standard continuous finite element basis functions are enriched with discontinuous functions that enable cracks or other material interfaces to be dynamically inserted at arbitrary locations in the mesh, which do not have to coincide with element boundaries.

XFEM has been implemented in the MOOSE framework, upon which BISON is based, using the phantom node technique. In the phantom node technique, elements containing discontinuities are split into two specially treated elements, and the mesh is connected in a way that properly represents discontinuities of the solution fields across the interface and continuity of fields on either side of the interface. A mesh-cutting algorithm was developed following the work of Richardson et al. (2011), with several key improvements that simplify it and facilitate modeling of crack branching. This has enabled a unique capability to solve arbitrary multiphysics problems with discontinuities represented with XFEM. This has been applied to a BISON simulation of fracture in ceramic nuclear fuel, represented using a 2-D cross-section model, during an initial ramp to full power. Discrete cracks are permitted to propagate in a mesh-independent manner based on physics-based criteria. Results from this simulation are shown in Figure 1.

\footnotetext{
${ }^{1}$ Duke University
} 


\section{NUCLEAR SCIENCE \& TECHNOLOGY}

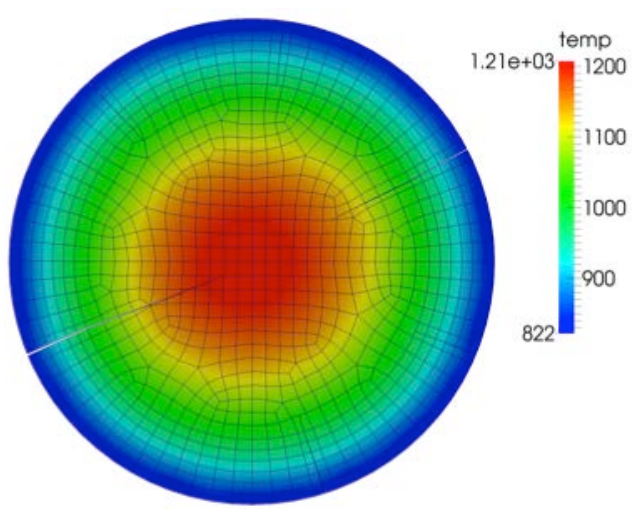

(a) Mesh with temperature (K) contours.

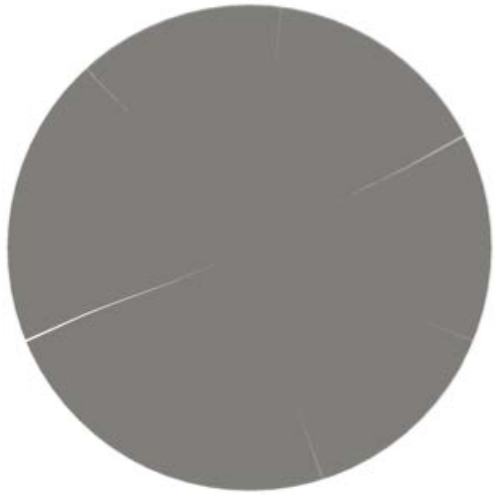

(b) Silouette of model showing radial fractures.

Figure 1. Results of a tightly coupled thermal/mechanical BISON simulation of radial crack formation in a fuel pellet after a ramp to a power of $25 \mathrm{~kW} / \mathrm{m}$.

DEM is a numerical modeling technique that represents a solid volume with a network of particles connected by mechanical bonds. A major strength of this method is that it is naturally able to represent arbitrary fracture by breaking those bonds once a failure criterion has been met. In this project, a series of proof-of-concept 2-D DEM simulations of fracture in nuclear fuel were performed using an existing research code. These simulations demonstrated the ability of a coupled thermal/mechanical DEM model to realistically capture the formation of radial cracks in nuclear fuel during an initial power-up. Figure 2 shows the crack patterns predicted by the DEM model at various power levels. The DEM model also predicted circumferential crack pattern formation during power-down. This work is documented in a recently published journal article. An initial, limited DEM capability has also been implemented in the BISON code. This will be expanded to permit DEM modeling of fuel to include the full set of fuel material and behavior models available in BISON.

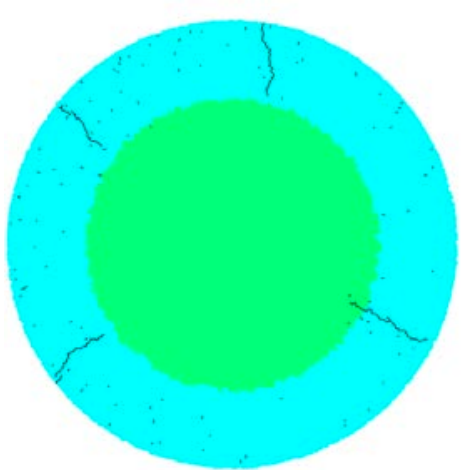

(a) Peak power=10 kW/m

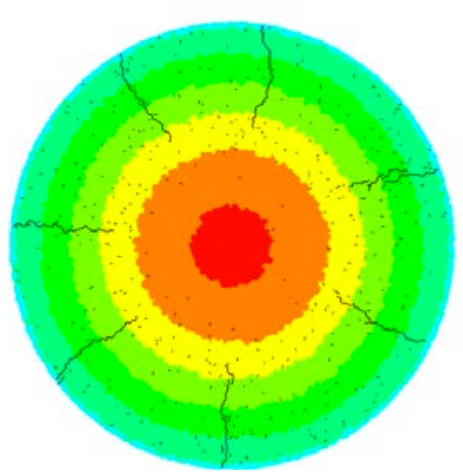

(b) Peak power $=25 \mathrm{~kW} / \mathrm{m}$

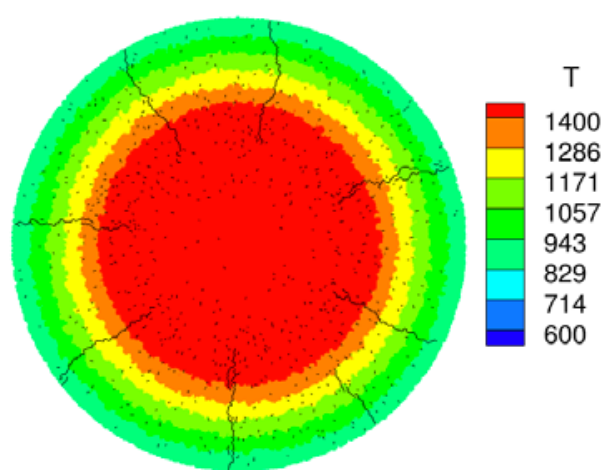

(c) Peak power $=40 \mathrm{~kW} / \mathrm{m}$

Figure 2. Results of 2-D DEM simulations of a fuel pellet cross section showing temperature $(K)$ and crack patterns (black lines) following initial power ramp. 


\section{Benefits to DOE}

This project has already demonstrated improved capabilities for fracture modeling in nuclear fuel. This will provide a stronger basis for predictive simulation of fuel designs and postulated accident scenarios that are outside the realm of applicability of methods currently in use. The fracture modeling techniques developed in this work are also directly applicable to modeling fracture in other nuclear power plant components, such as reactor pressure vessels and containment vessels. This will improve the safety and efficiency of nuclear power generation.

\section{Publications}

Huang, H., B. Spencer, and J. Hales, "Discrete Element Method for Simulation of Early-Life Thermal Fracturing Behavior in Ceramic Nuclear Fuel Pellets," Nucl. Eng. Des., Vol. 278, 515-528, October 2014.

\section{Presentations}

Spencer, B., H. Huang, J. Dolbow, and J. Hales, "Discrete Modeling of Early-Life Thermal Fracture in Ceramic Nuclear Fuel," In Proceedings of Water Reactor Fuel Performance Meeting 2014, Sendai, Japan, September 2014.

Spencer, B., "Advanced Fracture Modeling for Nuclear Fuel," Presentation of LDRD projects to DOE-NE program managers, Germantown, MD, August 25, 2014. 


\title{
13-097 - MOOSE Capability Extension In Support of Full Core Modeling
}

\author{
Derek Gaston, Cody Permann, David Andrs, John Peterson, and Andrew Slaughter
}

Nuclear reactor operation is inherently a multiscale, multiphysics phenomena. Large scale physics such as fluid flow and heat conduction are driven by atomistic effects of the fission process. Simulation of such a complex system is a daunting task. This project aims to add fundamental capabilities to MOOSE in support of multiscale nuclear reactor modeling. The main objectives center around three capabilities:

1. Link together applications

2. Deliver tools to the community

3. Provide a stable platform ensuring these complex capabilities can be easily developed and maintained.

\section{Summary}

The MOOSE Capability Extension In Support of Full Core Modeling LDRD has seen immense success in the first 2 years. During the first year of the project, advanced technical capability was added to the MOOSE multiphysics platform that enabled first-of-a-kind, full-core, multiscale nuclear reactor simulations by efficiently linking multiple MOOSE-based applications. That capability has been a showcase for the direction INL has taken for modeling and simulation.

The second year of the project was highlighted by large advances in software engineering practices to accelerate development of full-core simulation capabilities. In March of 2014, MOOSE was released as open source software, which has led to a rapid increase in both the number of users and the number of developers of MOOSE. MooseBuild, a new tool for coordinating development of multiple, interdependent MOOSE-based applications, was also created during the second year. A novel workflow for streamlining development of MOOSE-based applications was published in the "Working towards Sustainable Software for Science: Practice and Experiences" workshop. During the second year, the MOOSE project was also awarded an R\&D 100 award from R\&D Magazine, in large part because of the full-core simulation capability developed during this LDRD.

The third year will see all of the tools and capabilities of the previous 2 years fully utilized to deliver new insight through multiscale, full-core nuclear reactor modeling. Several of the systems used for these large-scale pilot runs in the first 2 years will be made more robust through enhancements and refactoring, further increasing usability..

\section{Benefits to DOE}

This project has benefited several DOE missions. This LDRD directly benefits energy resources as the capabilities produced are already being utilized to study advanced nuclear reactor and fuel concepts. These capabilities are also impacting environmental quality and science. The intrinsic capabilities being developed here are being applied to many disparate areas of environmental science and scientific endeavors (including fundamental material science studies). 


\section{Publications}

Gaston, D., C. Permann, J. Peterson, A. Slaughter, D. Andrs, Y. Wang, M. Short, D. Perez, M. Tonks, J. Ortensi, and R. Martineau, "Physics-Based Multiscale Coupling for Full Core Nuclear Reactor Simulation," Annals of Nuclear Energy, in press.

Slaughter, A., D. Gaston, J. Peterson, C. Permann, D. Andrs, and J. Miller, "Continuous Integration for Concurrent MOOSE Framework and Application Development on GitHub," Workshop on Sustainable Software for Science: Practice and Experiences 2 (WSSSPE2), New Orleans, LA, November 16, 2014.

Gaston, D., J. Peterson, C. Permann, D. Andrs, A. Slaughter, and J. Miller, "Continuous Integration for Concurrent Computational Framework and Application Development," Journal of Open Research Software, Vol. 2, 1-6, July 2014.

Short, M., D. Gaston, C. Stanek, and S. Yip, "A Perspective on Coupled Multiscale Simulation and Validation in Nuclear Materials," MRS Bulletin, Vol. 39, 71-77, 2014.

Gaston, D., C. Permann, D. Andrs, and J. Peterson, "Massive Hybrid Parallelism for Fully Implicit Multiphysics," In Proceedings of the International Conference on Mathematics and Computational Methods Applied to Nuclear Science and Engineering, Sun Valley, ID, May 5-9, 2013.

\section{Presentations}

Gaston, D., C. Permann, D. Andrs, J. Peterson, A. Slaughter, and R. Martineau, "MOOSE: Multiphysics Object Oriented Simulation Environment," EPRI Safety Technology Week, Charlotte, NC, June 2014.

Gaston, D., C. Permann, D. Andrs, J. Peterson, and A. Slaughter, "Physics Based Coupling for Multiscale Full Core Nuclear Reactor Simulation," SIAM Conference on Parallel Processing for Scientific Computing, SIAM PP14, Portland, OR, February 18-21, 2014.

Gaston, D., C. Permann, D. Andrs, J. Peterson, A. Slaughter, and R. Martineau, "MOOSE: Multiphysics Object Oriented Simulation Environment," NASA Langley Research Center, Newport News, VA, January 2014, invited presentation.

Gaston, D., C. Permann, D. Andrs, J. Peterson, and A. Slaughter, MOOSE Workshop, Texas A\&M University, College Station, TX, October 29-31, 2013.

Gaston, D., C. Permann, D. Andrs, J. Peterson, A. Slaughter, et al. "Full Core Reactor Simulation Using MOOSE," Closing Plenary, American Nuclear Society GLOBAL 2013, Salt Lake City, UT, Sept 29-October 3, 2013.

Gaston, D., C. Permann, D. Andrs, J. Peterson, and A. Slaughter, MOOSE Workshop, Commonwealth Scientific and Industrial Research Organisation (CSIRO), Perth, Australia, June 11-13, 2013. 
Gaston, D., C. Permann, D. Andrs, J. Peterson, A. Slaughter, et al. "Multiphysics, Multiscale Algorithms for Nuclear Power Applications," Plenary, International Conference on Mathematics and Computational Methods Applied to Nuclear Science and Engineering, Sun Valley, ID, May 5, 2013.

Gaston, D., C. Permann, D. Andrs, and J. Peterson, "Massive Hybrid Parallelism for Fully Implicit Multiphysics," SIAM Conference on Computational Science and Engineering, SIAM CS\&E13, Boston, MA, February 25-March 1, 2013. 


\title{
13-106 - Building Inorganic-Organic Hybrid Materials to Protect Metal Ion Sequestering Agents from Radiation-Induced Oxidative Damage
}

\author{
Peter Zalupski, Bruce Mincher, Michael Schultz, and Gary Buettner ${ }^{1}$
}

Radiolytic damage incurred to solvent extraction reagents during the process of hydrometallurgical recovery of actinides from used nuclear fuel has obstructed aqueous separations for decades. This LDRD project supports the back-end of nuclear fuel cycle through studies of the shielding properties of mesoporous carbon nanostructures, which will be utilized as encapsulating hosts for the metal ion complexing reagents, as illustrated in Figure 1. The inorganic (carbon)-organic (complexant) hybrid materials are tested for their ability to alleviate the damaging influence of free radicals produced when the energy of radioactive decay is deposited in matter. Shortly, the project seeks antioxidizing benefits of such hybrid capsules.
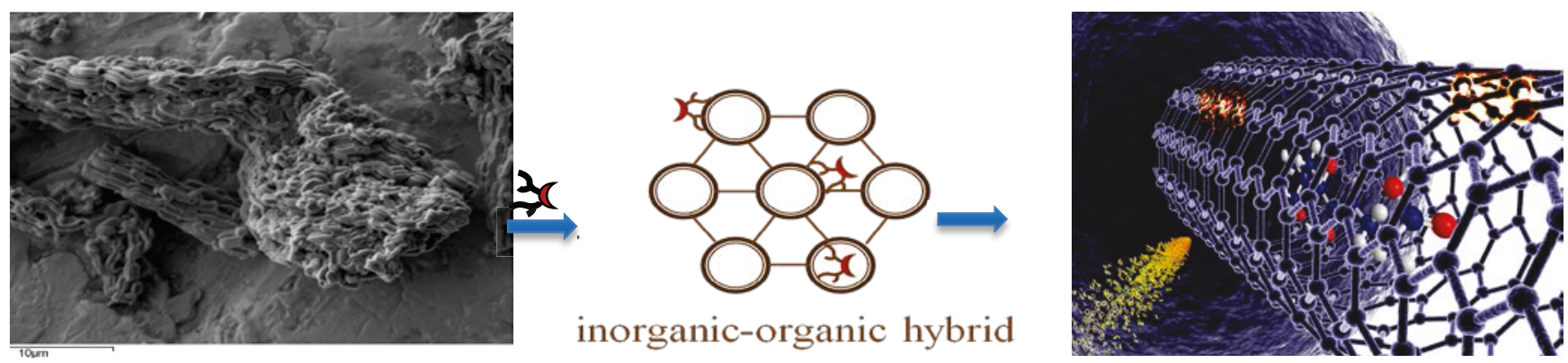

Figure 1. Pictorial representation of technical concept: the mesoporous carbon nanostructures, as shown by a scanning electron microscopy image on the left, are impregnated with a complexing agent represented by $\vec{x}$, entering the mesoporous channels. The constructed inorganic-organic hybrid offers shelter for an active reagent from free radical-induced oxidative damage, as illustrated on the image on the right.

Studies in FY 2013 proved the scientific concept proposed in this LDRD project. The metal ion coordination properties, when compared for the protected system (hybrid) and the unprotected one (reagent in solvent), revealed the antioxidizing benefit of the shielding mesoporous capsule.

\section{Summary}

Highlight accomplishments for FY 2014 are listed below:

A hybrid system where an organic reagent is a nitrogen heterocyclic structure (BTBP) has been successfully prepared. This compound, when employed in a liquid-liquid distribution system, exhibits very efficient coordination of trivalent actinides (e.g., americium $[\mathrm{Am}]^{3+}$ ) relative to analogous trivalent lanthanides (e.g., neodymium $[\mathrm{Nd}]^{3+}$ ). This differentiation between the two f-element groups is accomplished in a highly acidic environment, prompting this chemistry as one of the highly regarded options of hydrometallurgical recovery of minor actinides from mixtures of dissolved used nuclear fuel. Figure 2 summarizes the characterization studies performed on this

\footnotetext{
${ }^{1}$ University of lowa
} 
hybrid material. The expected trivalent actinide/trivalent lanthanide differentiation is maintained when the BTBP molecule is immobilized within the mesoporous carbon framework (Figure 2a). Significant kinetic effect in the coordination of $\mathrm{Am}^{3+}$ was noted, perhaps not surprisingly, judging from slow extraction kinetics exhibited by this chemistry in conventional liquid-liquid systems. Nonetheless, after the attainment of an equilibrium, the efficient actinide/lanthanide separation signals maintained a metal ion coordination activity. This is a necessary validation prior to any inquiry into the radioprotection concept.
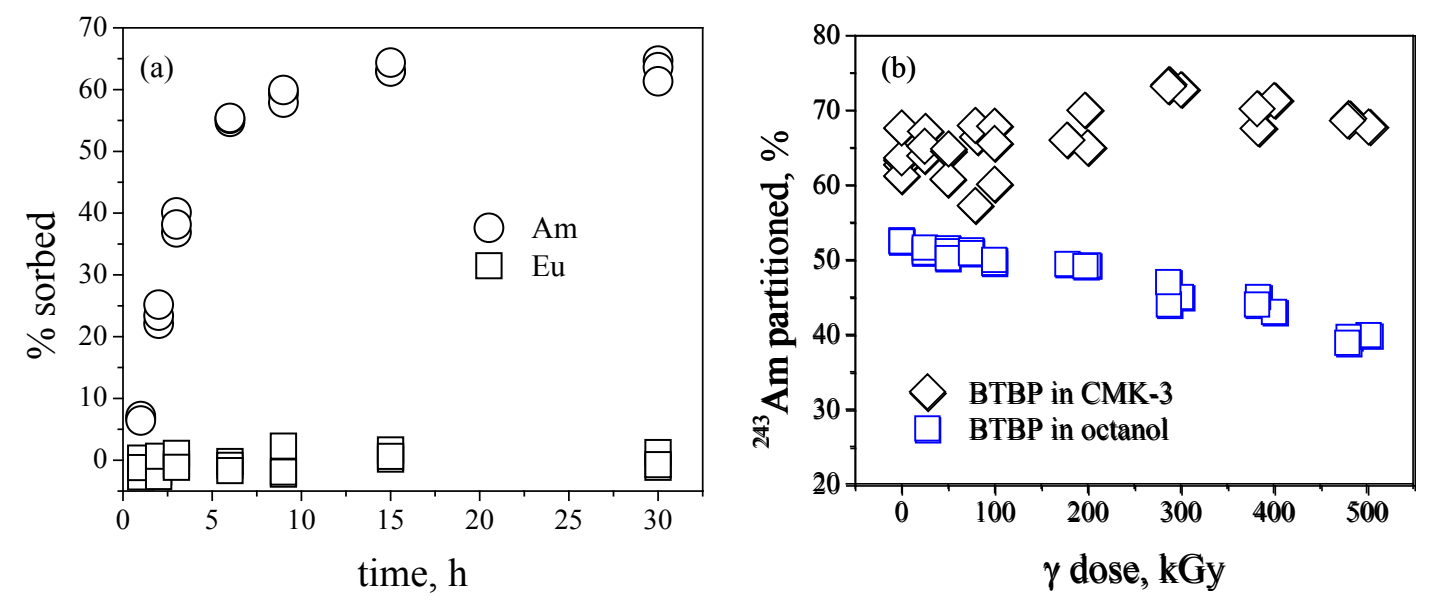

Figure 2. (a) Sorption of $\mathrm{Am}^{3+}$ and $\mathrm{Eu}^{3+}$ from $1 \mathrm{MHNO}_{3}$ by BTBP-containing hybrid material. (b) Effect of $\gamma$ radiation dose on the partitioning of $\mathrm{Am}^{3+}$ from $1 \mathrm{M} \mathrm{HNO}_{3}$ using two BTBP containing systems.

Figure $2 b$ summarizes the results of the irradiation study with the protected system (BTBP hybrid) and the unprotected one (BTBP in octanol). The results (although less convincing than those collected when studying a dialkylphosphoric acid reagent) confirm the benefits of reagent confinement in mesoporous carbon framework. Some gamma-induced degradation of the "unprotected" system is observed, whereas the efficiency of $\mathrm{Am}^{3+}$ coordination remains constant within the levels of uncertainty for the adsorption study.

With the successful demonstration of this concept for two inorganic-organic hybrid materials, the FY 2015 efforts will be geared towards the confinement of reagents of highest sensitivity to oxidation (i.e., dithiophosphinic acids).

\section{Benefits to DOE}

This project supports the feasibility studies on closing the nuclear fuel cycle where valuable materials locked inside used nuclear fuel are recovered and reused through further burning of fissionable isotopes.

Hydrometallurgical recovery of actinides from dissolved nuclear fuel is challenged by the radiolytic degradation of reagents used in aqueous separations systems. If protected, such reagents may function more effectively, improving the economics of such methodologies. 


\section{3-115 - Multi-scale Full Core Reactor Physics Simulation of the Advanced Test Reactor}

Mark DeHart, Yaqi Wang, Kord Smith, ${ }^{1}$ Jim Morel, ${ }^{2}$ Cristian Rabiti, Hongbin Zhang, Richard Martineau, and Barry Ganapol ${ }^{3}$

The INL ATR has a very complicated geometrical configuration that challenges most deterministic methods. However, accurate deterministic methods are necessary to be able to perform multiphysics simulations of the ATR. Coupled physics modeling will be used to improve quantification of safety margins for ATR and to support experiment design and analysis. Thus, the objective of this research is to develop a prototypic, multi-scale, multiphysics virtual reactor based on the ATR. Specifically, the research team is working to develop a neutron transport solver with RattleSNake, a neutron transport application built upon INL's MOOSE framework. A three-scale solution is proposed to provide an appropriately accurate solution based on the actual core geometry: fuel-resolved, plate-homogenized, and element-homogenized. This virtual reactor is to be used to support the mission of performance improvements together with continued safe and productive operation of the ATR.

\section{Summary}

Multiple scales require multiple levels of resolutions corresponding to different discretization schemes. At the initiation of this work, Rattle $S_{N}$ ake solved the radiation transport equation in the self-adjoint angular flux (SAAF) formulation discretized with a multigroup approximation in energy, a continuous finite element method (CFEM) in space, and a discrete ordinates $\left(S_{n}\right)$ approximation in angle. Over the first year of research, two new transport schemes were developed: the spherical harmonics expansion method $\left(P_{n}\right)$ and the least-squares $(L S)$ method with CFEM and $S_{n}$.

The $P_{n}$ method provides an alternative discretization scheme particularly for the plate-homogenized and element-homogenized scales. The $P_{n}$ implementation takes advantage of the components already present in

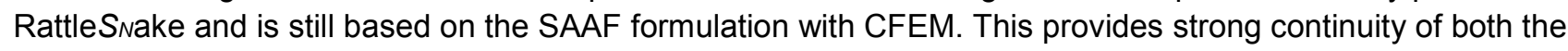
even and odd parity expansion moments with SAAF, which are different than the traditional parity-based $P_{n}$ methods. To our knowledge, this is the first multi-dimensional implementation of the $P_{n}$ method for the SAAF transport equation. The implementation of a $P_{n}$-based solver allows for novel comparisons, verification, and analysis between the $S_{n}$ and $P_{n}$ implementations as well as comparisons between the $P_{n}$ and analytical benchmarks. The implementation has been tested with benchmark problems, including the assembly-homogenized International Atomic Energy Agency 2-D pressurized water reactor benchmark. Convergence of the solution with increasing $P_{n}$ order has been observed, as expected.

Although the existing discretization scheme (SAAF-CFEM-SN) can treat void regions, it introduces an extra stabilization term that destroys the symmetry of the streaming and collision operator. A new form of a second-order, self-adjoint transport equation has been developed that has an advantage relative to SAAF-CFEM-SN in that the streaming and collision operator is symmetric positive definite. This equation is closely related to the standard LS or normal form of the transport equation. However, unlike the standard LS form

\footnotetext{
${ }^{1}$ Massachusetts Institute of Technology

${ }^{2}$ Texas A\&M University

${ }^{3}$ University of Arizona
} 
of the transport equation, this LS equation is compatible with source iteration. This derivation was implemented in

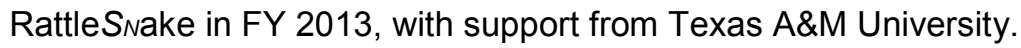

In FY 2014, the primary objective of this work was to develop and implement interface conditions for coupling scales on separate sub-domains on which different solution schemes may simultaneously apply, including different angular solutions using $S_{n}$ or $P_{n}$, a varying number of energy groups, and different levels of spatial resolution. Several objectives have been accomplished: (1) development of an unstructured mesh splitter; (2) development and implementation of Lagrange multipliers for the simple convection-collision equation, applied to the neutron transport equation; and (3) initial implementation of a mortar finite element method framework within RattleS Sake. Actualization of mortar finite element method-based methods within Rattle S Nake required deployment of a mortar finite element method architecture within the MOOSE framework itself. This modification to MOOSE was completed and initial work to apply this technology with RattleSNake solution algorithms is in progress.

Progress in FY 2014 also included improvements to individual discretization schemes in Rattle SNake. Significant progress has been made through development of two individual high-order transport schemes: the LS-SN-CFEM method and the SAAF-PN-CFEM method.

\section{Benefits to DOE}

The work completed to date has already been applied in initial modeling efforts for the INL Transient Test Reactor. Rattle $S_{N}$ ake has been coupled to BISON to provide temperature increases and distribution as a function of energy deposition required to simulate temperature feedback in transient operation. All three solution modes $\left(S_{n}, P_{n}\right.$, and diffusion) have been tested using a single Transient Test Reactor fuel element model in an infinite lattice. Efforts are ongoing to extend to a quarter-core representation. The multi-scale solution being implemented in RattleS $S_{N}$ ke will be imperative to be able to provide detailed transport and coupled multi-physics modeling with a Transient Test Reactor experiment vessel, driven by a lower order transport solution for the core itself.

\section{Publications}

Wang, Y., "A Remez Algorithm on Finding Polar Angle Quadrature for the Method of Characteristics," Proceedings of the 2015 ANS Annual Meeting - Nuclear Technology: An Essential Part of the Solution, San Antonio, TX, June 7-11, 2015, submitted.

Hansen, J., J. Peterson, J. Morel, J. Ragusa, and Y. Wang, "A Least-Squares Transport Equation Compatible with Voids," Journal of Computational and Theoretical Transport, DOI:10.1080/00411450.2014.927364, 2014.

Wang, Y., and F. Gleicher, "Revisit Boundary Conditions for the Self-Adjoint Angular Flux Formulation," PHYSOR 2014 - The Role of Reactor Physics toward a Sustainable Future, The Westin Miyako, Kyoto, Japan, September 28-October 3, 2014.

Sanchez, R., C. Rabiti, and Y. Wang, "Nonlinear Acceleration of a Continuous Finite Element Discretization of the Self-Adjoint Angular Flux Form of the Transport Equation," Nuclear Science and Engineering, Vol. 175, 213-226, November 2013. 
Wang, Y., "Nonlinear Diffusion Acceleration for the Multigroup Transport Equation Discretized with $\mathrm{S}_{\mathrm{n}}$ And Continuous FEM with RattleSNake," Proceedings of the 2013 International Conference on Mathematics and Computational Methods Applied to Nuclear Science \& Engineering (M\&C 2013), Sun Valley, ID, May 59, 2013.

\section{Presentations}

DeHart, M., F. Gleicher, J. Ortensi, and Y. Wang, "Methods and Validation to Improve the Operational Efficiency and Expand Testing Regimes of the Iransient Reactor Experiment And Iest (TREAT) Facility," NEAMS PI Meeting, Washington, D.C., December 2014.

DeHart, M., "MAMMOTH: A Next Generation Reactor Physics and Fuel Management System," INL/MIS-14-31761, DOE NE Advanced Modeling and Simulation (NEAMS) briefing at INL, August 21, 2014.

DeHart, M., "Multi-Scale Full Core Reactor Physics Simulation of the Advanced Test Reactor," INL/MIS-14-31761, Briefing on LDRD 13-115 for visitors from DOE: DOE-NE Senior Leadership and Program Managers, May 7, 2014. 


\title{
13-121 - Advanced In-Situ Measurement Techniques in TREAT
}

\author{
J. Keith Jewell, Tony Hill, and Eric Burgett ${ }^{1}$
}

This LDRD explores Transient Reactor Test Facility (TREAT) configurations that can facilitate high-resolution, in situ measurements on small nuclear material samples and the feasibility of mating current microstructure measurement technologies and techniques to the new experimental environment. The in situ microstructure measurements can be performed without impacting the TREAT restart or the nominal mission of the TREAT program and effectively extend the TREAT mission to include beginning of life microstructure separate effects measurements.

During the first year, the focus was on establishing a baseline configuration for utilizing TREAT for science-based microstructure studies in a way that would not impact the core mission of transient fuel testing for qualification. This effort provided a proof of concept design for delivering advanced science-based separate effects data on fuel performance and underlying phenomena.

A neutronics modeling and simulation analysis was performed to determine the feasibility and baseline requirements for in situ irradiations that could provide high-fidelity imaging and microstructure characterization of fuel samples within the modified TREAT configuration. The analysis produced a design of an irradiation capability utilizing the unused east thermal column of TREAT for performing separate effects and microstructure studies. In this way, both the macroscopic and the microstructure behavior of the fuel can be studied simultaneously under the same power profile, as well as taking full advantage of the significant "off-cycle" time between transients when TREAT is in a low power steady state. The unique pulse structure of TREAT allows high-resolution imaging in between moderate power pulses, such that the microstructure evolution of fuel materials can be studied as a step-cycle process.

The second year focused on the data handling and imaging algorithms needed to collect in situ data on large samples. Unique to this approach is the potential for simultaneous acquisition of microstructure evolution data from hundreds of features within a single fuel material sample. The challenge comes in automating the identification of salient features that directly impact and inform the modeling and simulation efforts. An additional challenge is the sheer amount of data from acquiring imaging at multiple, high resolutions that can be cross correlated with simulation results.

\section{Summary}

The TREAT reactor was modeled in an unmodified configuration to compare the impact of the proposed irradiation cavity on the base operations of the TREAT reactor. Four group cross-section neutron contour maps were produced for both the main reactor core as well as the science-based irradiation system integrated into the TREAT east thermal column.

Both low-enriched uranium $(U)$ and highly enriched $U$ irradiation cavities were investigated to optimize the utility of the microstructure characterization capability while minimizing the impact to nominal TREAT transient testing. It was determined that the previously unused thermal column on the east side of the reactor vessel offered sufficient neutron flux and access for sample movement and imaging equipment to develop a parasitic microstructure

\footnotetext{
${ }^{1}$ Idaho State University
} 
characterization component to TREAT operations. There is adequate space for a specially designed scanning electron microscope system in this location. With a low-enriched $U$ designed system, an integrated fast neutron flux of $\mathrm{E} 12 \mathrm{n} / \mathrm{cm}^{2} / \mathrm{s}$ is achievable while operating TREAT in the $100 \mathrm{~kW}$ steady state mode. This would allow for a collection of microstructure data continually whenever TREAT is not being used for transient testing.

A similar neutronics analysis was performed for a highly enriched $U$ boosted irradiation cavity in the thermal column in order to achieve an E13 $\mathrm{n} / \mathrm{cm}^{2} / \mathrm{s}$ integrated flux in the microstructure cavity while operating TREAT in the $100 \mathrm{~kW}$ steady state mode. Full TREAT core flux profiles were produced for a number of different microstructure characterization irradiation scenarios. In addition, a safety envelope was produced to ensure that normal transient testing would not be limited by the inclusion of the irradiation cavity in the east thermal column. It was determined that the irradiation cavity can operate indefinitely in the steady state mode, and cooling was designed to ensure that the hottest fuel element can survive a 0.5 second $16 \mathrm{GW}_{\text {th }}$ pulse in the transient operation. Using an adiabatic boundary with conduction to the coolant flow only, a computational fluid dynamics code that is part of the Fluent and Solidworks package was used to model the hottest fuel element, showing a $53^{\circ} \mathrm{C}$ temperature rise in the hottest spot.

An additional data acquisition scenario was investigated to take advantage of the unique pulse structure available at TREAT. The highly enriched $U$ cavity provides $1 \times 10^{15} \mathrm{n} / \mathrm{cm}^{2} / \mathrm{s}$ when TREAT is operated at less than $10 \mathrm{MW}$, which it can do for a maximum of approximately 200 seconds with a 60 minute cooling time in between pulses. The equivalent fast reactor dose can be delivered in less than 5 days with high-resolution images collected at the end of each pulse.

\section{Benefits to DOE}

Development of these capabilities will make INL a leader in the science-based characterization of the time evolution of microstructural fuel behavior during irradiation. The proposed work leverages the unique TREAT facility and moves the facility into the realm of a science-based understanding for fuels development. Successful completion of the project would position the TREAT facility as the premier test site for both beginning of life microstructure fuels testing as well as end of life transient testing.

The work funded by this LDRD is in line with the larger strategy developed by DOE-NE to shift the paradigm of advanced nuclear fuels development to ensure the nation's energy security for the future. The historical approach of relying on experiential models and extensive qualification testing makes development of new fuels and advanced reactors a very time consuming and expensive process. Separate effects and isolated effects testing will inform new first principle models to develop a predictive performance capability as fuels research moves forward. This project, in particular, leverages the necessary TREAT investments for fuel qualification and takes advantage of the enormous advances in computing methods and resources.

\section{Publications}

A journal article is being drafted to detail the TREAT neutronics analysis. A second article will be written to demonstrate the data handling and analysis capable with the TREAT pulse structure. 


\title{
14-009 - Development of an INL Capability for High
} Temperature Flow, Heat Transfer, and Thermal Energy Storage with Applications in Advanced Small Modular Reactors, High Temperature Heat Exchangers, Hybrid Energy Systems, and Dynamic Grid Energy Storage Concepts

\author{
James E. O'Brien, Piyush Sabharwall, and Xiaodong Sun ${ }^{1}$
}

Advanced reactor systems require R\&D, testing, and qualification for critical systems, structures, and components. To meet these requirements, a highly versatile high-temperature test facility is needed. This LDRD project was approved to initiate development of such a capability. Key activities include (1) qualification and testing of critical components in a prototypical high-temperature, high-pressure environment, (2) materials development and qualification, (3) manufacturer and supplier evaluation and development, and (4) fundamental thermal hydraulics research and associated verification and validation.

The initial phase of this project was focused on the development of a conceptual design for a high-temperature, multi-fluid, multi-loop test capability to support heat transfer, flow, materials, and thermal energy storage (TES) research for nuclear and nuclear-hybrid applications. In its initial configuration, this will include a high-temperature helium (He) loop, a liquid salt loop, and a hot water/steam loop. The three loops will be thermally coupled through an intermediate heat exchanger (IHX) and a secondary heat exchanger. Research topics to be addressed include the characterization and performance evaluation of candidate compact heat exchangers such as printed circuit heat exchangers at prototypical operating conditions, flow and heat transfer issues related to core thermal hydraulics in advanced He-cooled and salt-cooled reactors, and evaluation of corrosion behavior of new cladding materials and accident-tolerant fuels for light water reactors at prototypical conditions. Based on its relevance to advanced reactor systems, the new capability has been named the Advanced Reactor Technology Integral System Test (ARTIST) facility.

The He loop will be designed for high-temperature operation up to $750^{\circ} \mathrm{C}$ at a pressure of $7 \mathrm{MPa}$. The He loop will provide high-temperature process heat to the liquid salt intermediate heat transfer loop via an IHX. The baseline design for this heat exchanger will be a high-efficiency compact microchannel printed circuit heat exchanger with a nominal heat duty of $55 \mathrm{~kW}$. The He loop will include a He-He recuperator, also based on a printed circuit heat exchanger design. The He loop will have a high-temperature test section for heat transfer and materials studies. Research topics that may be studied in the high-temperature He test section include flow distribution, bypass flow, heat transfer in prototypical prismatic core configurations under forced and natural circulation conditions, parallel flow laminar instability during pressurized cooldown, and turbulent heat transfer deterioration. Oxidation effects associated with water or air ingress could also be examined.

The intermediate heat transfer loop will utilize a high-temperature liquid salt as a heat transfer fluid. Liquid salts have been identified as excellent heat transport fluids for intermediate loops, supporting several types of advanced high-temperature reactors. Liquid salts have also been proposed for use as a primary coolant for the Advanced High Temperature Reactor and the Fluoride Salt-Cooled High-Temperature Reactor. The high-temperature test section in the liquid salt loop can be used for examination of materials issues, thermal

\footnotetext{
${ }^{1}$ Ohio State University
} 


\section{NUCLEAR SCIENCE \& TECHNOLOGY}

stresses, and high-Prandtl-number heat transfer. The liquid salt loop will include a TES system for support of thermal integration studies. The TES system will be based on freezing and melting of the salt acting as a high-temperature phase change material.

The high-temperature test section in the steam/water loop will be used primarily for prototypical evaluation of new cladding materials and accident-tolerant fuels. It will be designed to characterize the thermal, chemical, and structural properties of candidate advanced fuel cladding materials and designs under various simulated flow and internal heating conditions to mimic operational reactor conditions prior to in-reactor testing. Careful control of water chemistry will be essential for these studies; a water chemistry control section is included in the design of the loop.

A 3-D computer-aided design model of the ARTIST experimental testing capability has been developed using Pro-Engineering software. The computer-aided design model includes geometrically accurate representations of all of the major components and piping. A rendering of the model is provided in Figure 1.

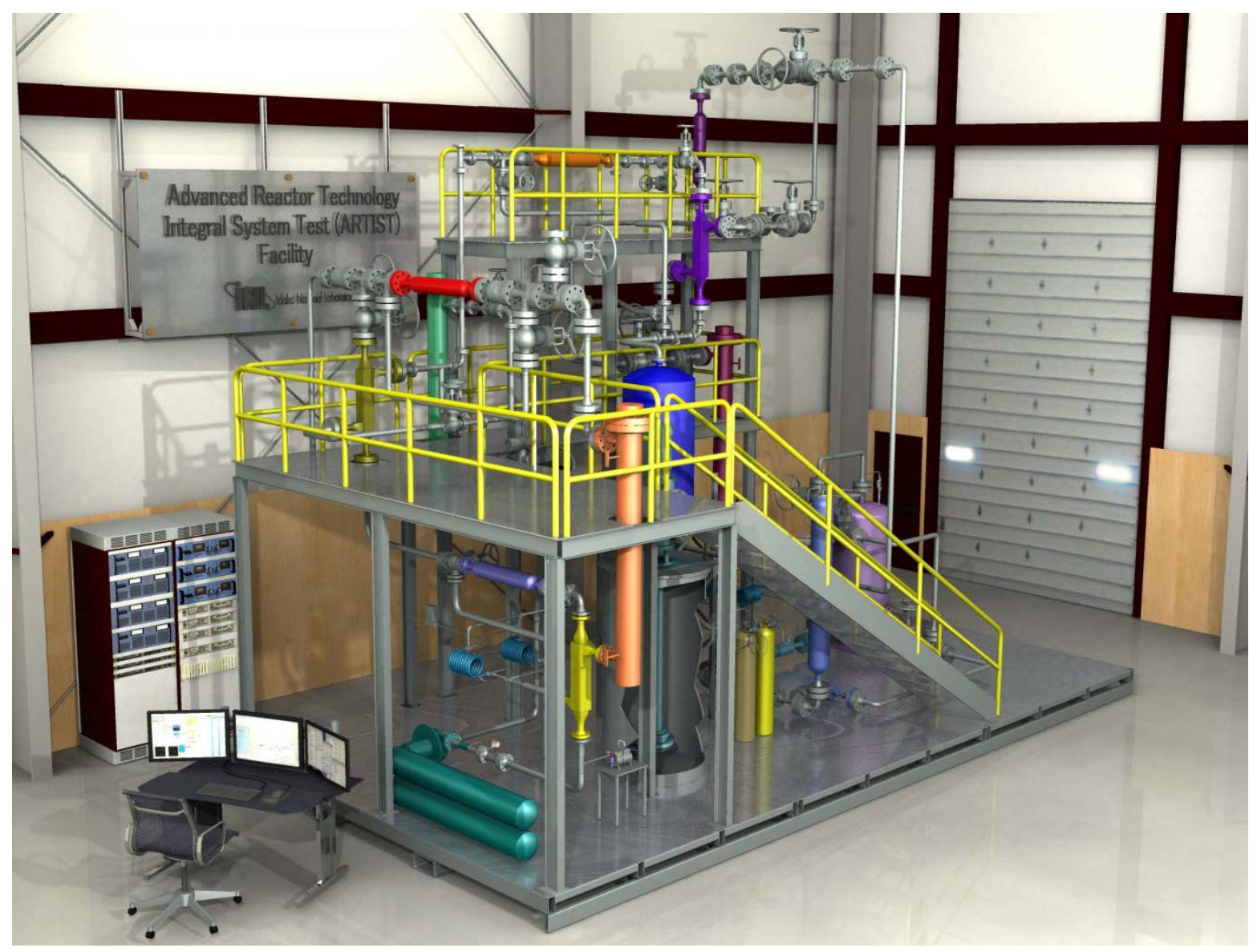

Figure 1. Rendering of a computer-aided design model showing the ARTIST capability in the INL Energy Systems Laboratory (ESL). 


\section{Summary}

A detailed conceptual design of a high-temperature, multi-fluid, multi-loop testing capability to support heat transfer, flow, materials, and TES research for nuclear and a nuclear-hybrid application has been completed. The capability includes three flow loops: a high-temperature He loop, a liquid salt loop, and a hot water/steam loop. The three loops will be thermally coupled through an IHX and a secondary heat exchanger. All major system components have been identified with required performance and materials specifications. Primary system engineering and research test objectives have been identified. A high-temperature phase-change TES system is included for support of thermal integration studies with co-located hybrid energy systems. For this purpose, process feed and process return lines have been included on the TES and on the hot water loop.

\section{Benefits to DOE}

This program supports research, development, and demonstration activities designed to promote safety, technical, economical, and environmental advancements of innovative Generation IV nuclear energy technologies. The ARTIST capability will provide a physical test platform for performance and integrity evaluation of Advanced Reactor Technology-relevant components at prototypical conditions. Using the ARTIST facility, the technology readiness level of important components such as the IHX can be significantly advanced. The facility will also support fundamental research in three high-temperature test sections for a variety of test and verification and validation objectives.

\section{Publications}

Sabharwall, P., J. O'Brien, and S. Yoon, "Experimental Facility for Development of High-Temperature Reactor Technology: Instrumentation Needs and Challenges," International Congress on Advances in Nuclear Power Plants, ICAPP, Nice, France, May 3-6, 2015, accepted.

O'Brien, J., P. Sabharwall, and S. Yoon, "Development of a Multi-Loop Flow and Heat Transfer Facility for Advanced Nuclear Reactor Thermal Hydraulic and Hybrid Energy System Studies," ASME International Congress and Exposition, Montreal, Quebec, November 14-20, 2014.

O'Brien, J., P. Sabharwall, and S. Yoon, "A Multi-Purpose Thermal Hydraulic Test Facility For Support of Advanced Reactor Technologies," ANS 2014 Winter Meeting and Nuclear Technology Expo, Anaheim, CA, November 9-13, 2014.

O’Brien, J., P. Sabharwall, S. Yoon, and G. Housley, "Strategic Need for a Multi-Pupose Thermal Hydraulic Loop for Support of Advanced Reactor Technologies," INL/EXT-14-33300, October 2014.

\section{Presentations}

"Nuclear Energy in the Context of Today's Energy Challenges," Nuclear Engineering Seminar Series, The Ohio State University and University of Cincinnati, Columbus, OH, March 26, 2014. 


\title{
14-010 - Use of Linear Variable Differential Transformer (LVDT)-Based Methods to Detect Real-Time Geometry Changes during Irradiation Testing
}

\author{
Darrell Knudson, Kurt Davis, Keith Condie, Joy Rempe, John Crepeau, ${ }^{1}$ and Steinar Solstad ${ }^{2}$
}

New materials are being considered for fuel, cladding, and structures in advanced and existing nuclear reactors. These materials can undergo significant dimensional and physical changes during irradiation. However, in the U.S., the only method for currently detecting such changes is by repeatedly irradiating a sample for a specified period of time and then removing it from the reactor for evaluation. The time and labor to remove, examine, and return irradiated samples for each measurement makes this approach very expensive. In addition, such techniques provide limited data, and handling may disturb the phenomena of interest. These problems are compounded by the fact that capabilities to conduct this type of measurement are not widely available.

For these reasons, in-pile detection of changes in geometry is greatly needed to understand real-time behavior during irradiation testing of fuels and materials in high flux U.S. Material and Test Reactors. This project will enable the ATR to deploy advanced Linear Variable Differential Transformer-based test rigs capable of detecting in-core, real-time changes in length and diameter of fuel rods or material samples. In particular, this new INL capability will enable detection of changes in diameter due to crud deposition or pellet-clad interactions during fuel irradiation testing in addition to changes in elongation due to creep. A new advanced test rig will be designed and evaluated that combines an enhanced Linear Variable Differential Transformer jointly developed by INL's High Temperature Test Laboratory (HTTL) and Institute for Energy at the Halden Reactor Project (IFE), a diameter gauge developed by IFE, and a controlled load elongation rig inspired by the Technical Research Center of Finland. Insights and results from test rig evaluations completed by INL and University of Idaho researchers using existing HTTL autoclaves will be used to develop a final test rig design for use in the ATR. This new capability will provide vital support for DOE programs, such as the ATR National Scientific User Facility, Fuel Cycle R\&D, Nuclear Energy Enabling Technology, and the Next Generation Nuclear Plant programs.

\section{Summary}

Two technical tasks were defined to accomplish the objectives of this 3 year project: Task 1 is focused on developing and evaluating (in a laboratory setting) a diameter gauge test rig for ATR irradiations; Task 2 is focused on developing and evaluating (in a laboratory environment) a test rig where the diameter gauge (from Task 1 ) is combined with controlled load testing capabilities. The first year of this project was devoted to designing, procuring, fabricating, and initiating bench top testing to evaluate the performance of a diameter gauge test rig. The design of the diameter gauge test rig was completed (see Figure 1). In addition, IFE completed final testing and calibration of the diameter gauge, associated electronics, and a guiding system and shipped these items to INL for testing (see Figure 2). During this year, INL also initiated laboratory testing and preparations for autoclave testing (Figure 3). These evaluations are essential for ensuring that the diameter gauge will perform with the required accuracy and reliability prior to deployment in an ATR pressurized water reactor loop.

\footnotetext{
${ }^{1}$ University of Idaho

${ }^{2}$ Halden Reactor Project
} 


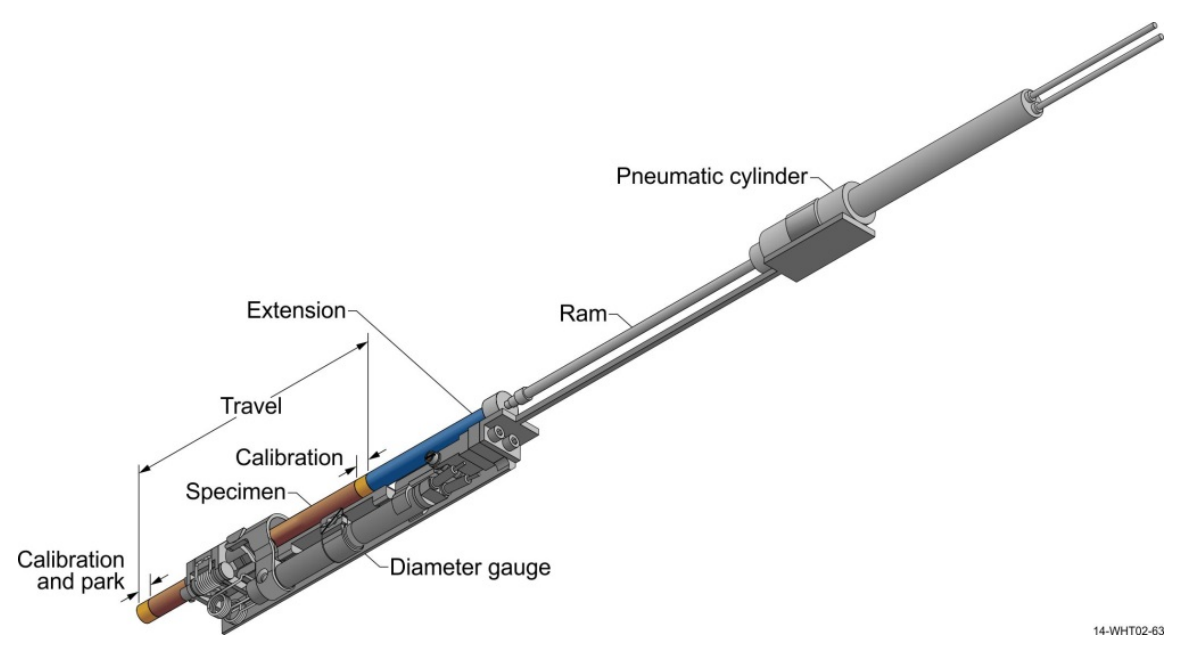

Figure 1. Conceptual design of HTTL-designed diameter gauge test rig proposed for ATR.

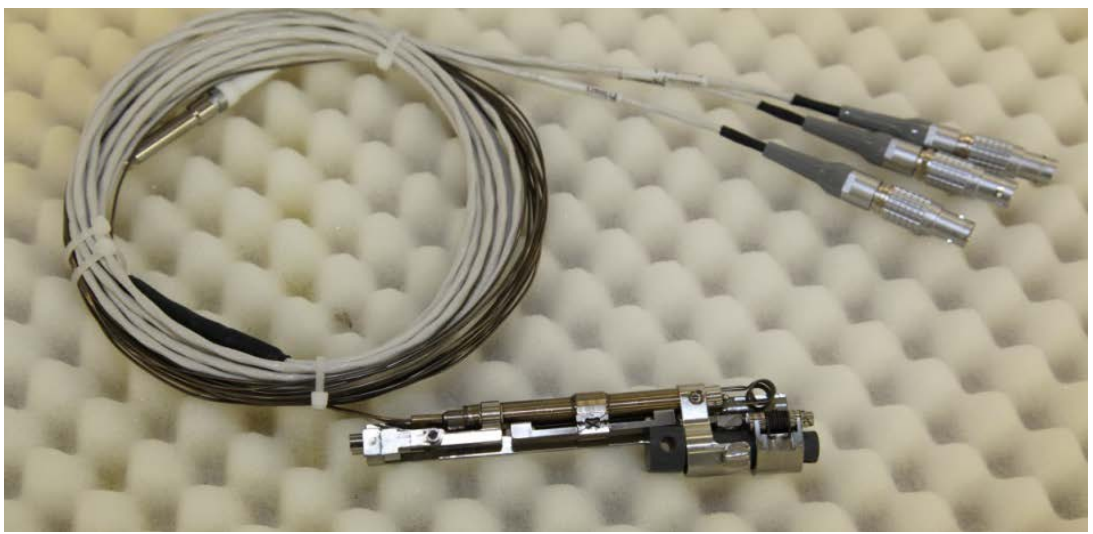

Figure 2. Diameter gauge with associated hardware.
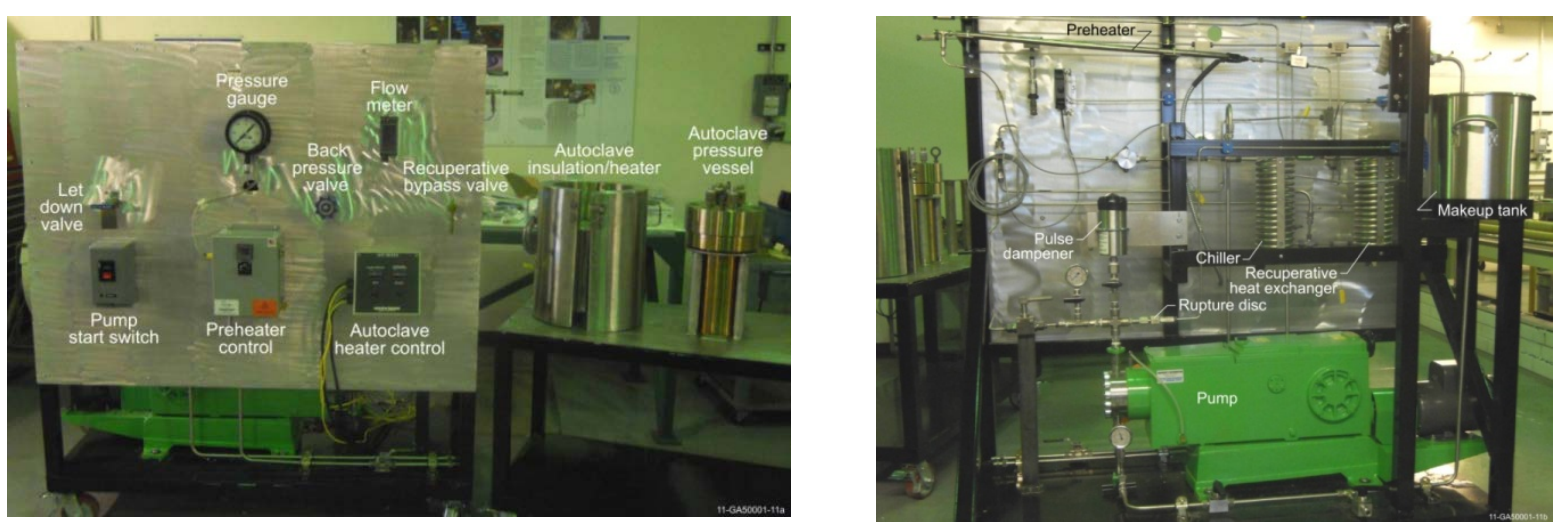

Figure 3. HTTL autoclave with controls for heating water and sampling water chemistry in which the diameter gauge test rig will be evaluated. 
In addition, a journal article was prepared and submitted to Nuclear Technology, and an abstract was prepared and submitted for presentation at the 9th International Conference on Nuclear Plant Instrumentation, Control \& Human-Machine Interface Technologies.

This research also funded an INL employee completing a University of Idaho graduate degree. In addition, this research helps grow the in-pile instrumentation capability established at INL's HTTL.

\section{Benefits to DOE}

Development of advanced in-core instrumentation is critical to DOE's energy security mission to provide world-class facilities for advancing nuclear science and technology. Successful completion of this effort will result in a proven test rig that can be used by DOE researchers and industry organizations to obtain real-time data for diameter changes in fuel and material specimens during irradiation testing in U.S. high flux Materials Testing Reactors, such as ATR and the Transient Test Reactor facility when it is restarted.

\section{Publications}

Rempe, J., D. Knudson, J. Daw, T. Unruh, B. Chase, K. Davis, A. Palmer, and R. Schley, "Advanced In-pile Instrumentation for Materials Testing Reactors," IEEE Transactions on Nuclear Science, Vol. 61, 1984-1994, August 2014.

Davis, K., D. Knudson, and J. Rempe, "LVDT-based Test Rigs for use in MTRs," Nuclear Technology, submitted June 26, 2014.

\section{Presentations}

Davis, K., D. Knudson, K. Condie, and J. Rempe, "Measurement of Diameter Changes during Irradiation Testing," 9th International Conference on Nuclear Plant Instrumentation, Control \& Human-Machine Interface Technologies (NPIC \& HMIT 2015), Charlotte, NC, February 21-26, 2015.

Carmack, J., and J. Rempe, "Overview of DOE Advanced In-Pile Instrumentation Research and Development," U.S.-Russia Civil Nuclear Energy Cooperation Fuels and Materials Working Group Meeting, Dimitrovgrad, Russia, October 23-25, 2013. 


\title{
14-025 - Minor Actinide and Lanthanide Separations in Alternative Media
}

\author{
Mitchell Greenhalgh and R. Scott Herbst
}

Since its inception, the nuclear industry, specifically the reprocessing of irradiated nuclear fuels, has realized that the separation of minor actinides (MA) (e.g., americium [Am] and curium [Cm]) from lanthanides (LN) and their subsequent recycle into new reactor fuel would greatly reduce the radiotoxicity of the waste generated by reprocessing. However, the separation of MA from LN represents one of the most daunting challenges faced by modern day separation scientists, rivaled in complexity only by isotopic separations. The LN/MA separation is difficult due to the remarkably similar chemical behavior between the $L N$ series (which includes the elements of lanthanum [La] through lutetium [Lu] and commonly yttrium $[\mathrm{Y}]$ ) and the MA. The ultimate goal of this project is to develop and test efficient solvent extraction (SX) flowsheets and equipment for the separation of MA from LN elements in alternative media (i.e., chloride, sulfate, phosphate, or thiocyanate). Traditional nuclear processing separations have focused on nitrate media $\left(\mathrm{HNO}_{3}\right)$, and the process equipment (notably constructed from stainless steel) is compatible only with the nitrate media. Industrially, the separation and purification of the LN species typically utilizes chloride media $(\mathrm{HCl})$ and different extractants and diluents than those indigenous to the nuclear industry. Ironically, most of these industrially proven systems have not been evaluated for MA/LN separation despite the inordinately similar chemistry of these elements in solution. Laboratory experiments will be performed to establish the fundamental separations behavior of MA and LN in alternative systems. Experimentally acquired data will be used to develop and test process flowsheets for the most promising separation schemes. Additionally, chemically compatible SX equipment suitable for use in a glove box or hot cell will be designed, procured, and tested for the new flowsheets with actual radionuclides of interest in laboratory hoods at tracer levels.

\section{Summary}

Laboratory experiments to determine the fundamental separation characteristics and behaviors of MA and LN utilizing alternative extractants and non-traditional aqueous media were performed during the first year of this project. A series of batch contact experiments were performed with three alternative extractants (i.e., Cyanex 923, lonquest 801, and Cyanex 572) in chloride, nitrate, and thiocyanate media to determine the LN/MA separation factors in these systems and compare those separations to each other. The highest separation factor for europium (Eu) and Am was found in a chloride-based media with the lonquest 801 extractant. Although separations of Eu from Am were also achieved in the other investigated systems, the chloride/lonquest system was by far the most promising separation factor obtained to date. These favorable results will narrow the focus of additional laboratory testing in the coming year to the chloride/lonquest system, and the data obtained from these evaluations will be utilized to develop a full flowsheet for demonstration in chemically compatible SX equipment.

In addition to the laboratory separations testing, a supplier of small, laboratory-scale SX was identified. This supplier manufactures small mixer-settler equipment that is constructed of a Kynar $\circledast$ polymer, which is chemically compatible with all of the proposed alternative medias in this project and is also the most radiation-resistant plastic available. During the year, 32 stages of these mixer-settlers were procured and received from Metallextraktion $A B$ of Sweden. The mixer-settlers have a maximum throughput of $166 \mathrm{~mL} / \mathrm{min}$ and a total solution hold up of only $600 \mathrm{~mL}$ per stage. The equipment can be operated in a variety of process configurations, 
which will ultimately be defined by the concurrent laboratory evaluations. The entire 32-stage system only requires approximately 12 linear feet of bench space, making it ideal for testing in a space-limited radiological environment. The required pumps and connective tubing for the system were also acquired during the year. Initial hydraulic evaluations of the mixer-settler equipment were performed to define the operating parameters that would achieve the desired separations (i.e., maximum flowrates, mixing speeds, and mass transfer efficiencies). The mixer-settlers will initially be set up in a non-radiological laboratory where additional testing and system configurations can be performed prior to radiological evaluations.

\section{Benefits to DOE}

The chemistry and engineering expertise developed and separations processes being examined are directly relevant to INL's nuclear fuel cycle mission-in particular, the development of separation capabilities that can be applied to processes different from the norm. The equipment and engineering developments related to process flowsheets are also directly relevant to future nuclear fuel cycle separations. Furthermore, the unique separations capabilities and equipment obtained in this project represent the only location in the United States where such work can be performed. The technical products (journal articles and patents) generated during this project will benefit the advanced separations reputation of INL. 


\title{
14-026 - Multiscale Modeling on Delayed Hydride Cracking in Zirconium: Hydrogen Transport and Hydride Nucleation
}

\author{
Yongfeng Zhang and Xianming Bai
}

Zirconium ( $\mathrm{Zr}$ ) based alloys are the main cladding materials in current light water reactors due to their low neutron absorption cross-section, superior mechanical properties, and good corrosion resistance. However, they suffer from hydrogen $(\mathrm{H})$ embrittlement, in particular delayed hydride cracking $(\mathrm{DHC})$, due to hydride formation and reorientation under stress. Delayed hydride cracking is one of the major safety concerns during used fuel storage. Fundamental understanding on how a hydride nucleates, which requires knowledge of $\mathrm{H}$ transport and segregation, is a prerequisite for predictive modeling of hydride formation. Such information is currently missing.

The objective of this project is to improve the current understanding on how $\mathrm{H}$ transports and how hydride nucleates in a hexagonal close packing (hcp) $\alpha$-Zr matrix. For these purposes, the two main tasks include (1) investigating $\mathrm{H}$ diffusion and segregation (interaction with lattice defects) in $\alpha-\mathrm{Zr}$ using molecular dynamics (MD) simulations and (2) developing an atomic kinetic Monte-Carlo model for hydride growth. The results will advance our knowledge on $\mathrm{H}$ transport and the early stage nucleation and growth of hydrides. The data collected from the atomistic scale will be useful for upper scale modeling on hydride formation and reorientation.

\section{Summary}

This LDRD is in its first fiscal year. In FY 2014, we focused on thermodynamical properties of hydrides and kinetic properties of $\mathrm{H}$ transport in $\alpha-\mathrm{Zr}$ using MD simulations. H segregation at lattice defects and interfacial properties between hydride and $\alpha-Z r$ have also been studied.

Using the charge-optimized many-body (COMB) potential, newly developed by our collaborators at the University of Florida, the formation energy of hydrides with various $\mathrm{H} / \mathrm{Zr}$ ratios is calculated. The results are in good agreement with previous density functional theory (DFT) calculations and experiments, as shown in Figure 1(a). We found that charge transfer between $\mathrm{H}$ and $\mathrm{Zr}$ may further stabilize hydrides. Turning on charge transfer in the COMB potential lowers the formation energy and thus increases the stability (Figure 1[a]) of hydrides. In the hydride phases, $\mathrm{H}$ has a charge state of about -0.21 , and the charge state of $\mathrm{Zr}$ atoms varies with their local coordination.

Using the same COMB potential, the structure of coherent and non-coherent $\{111\} \mathrm{fcc} /\{0001\} \mathrm{hcp}$ interfaces between $\varepsilon$-hydride $\left(\mathrm{ZrH}_{2}\right)$ and $\alpha-\mathrm{Zr}$ are characterized. For the coherent interface, the system is highly strained, and the interfacial energy is not distinguishable from the strain energy. The normalized formation energy of hydride increases with hydride thickness, suggesting a lower strain energy at the nucleation stage than that in bulk phase. For the non-coherent interface, mismatch dislocations are present after relaxation. Current efforts are focused on extracting an interfacial energy that can be used in upper scale simulations.

The COMB potential predicts preferential $\mathrm{H}$ segregation at stacking faults with the segregation energy of about $0.5 \mathrm{eV}$ lower than that in bulk $\alpha-\mathrm{Zr}$, as shown in Figure 1(b). This result is in agreement with previous DFT calculations in literature. Moreover, some preliminary results show preferential $\mathrm{H}$ segregation may also occur at radiation-induced $<c>$ loops. In hcp metals, radiation-produced self-interstitial atoms may form prismatic loops with the $<c>$ component in the Burgers vector. These loops may take the extrinsic or intrinsic stacking fault structure inside the loop interior. MD simulations with the COMB potential show that the segregation energy of 
$\mathrm{H}$ can be significantly lowered in the loop interior due to the stacking fault configuration and the stress field of loops. As the stacking fault or $<c>$ loops take a face-centered cubic local structure, preferential segregation of $\mathrm{H}$ may facilitate nucleation of hydrides. This effect will be further studied in FY 2015.

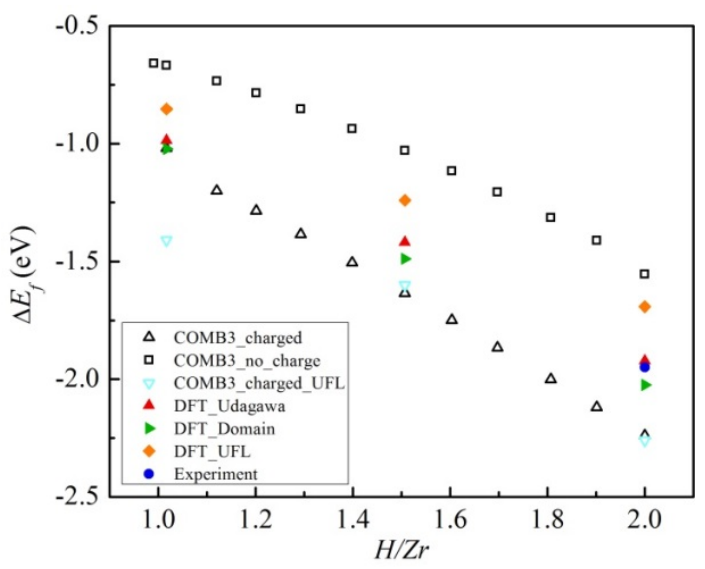

(a)

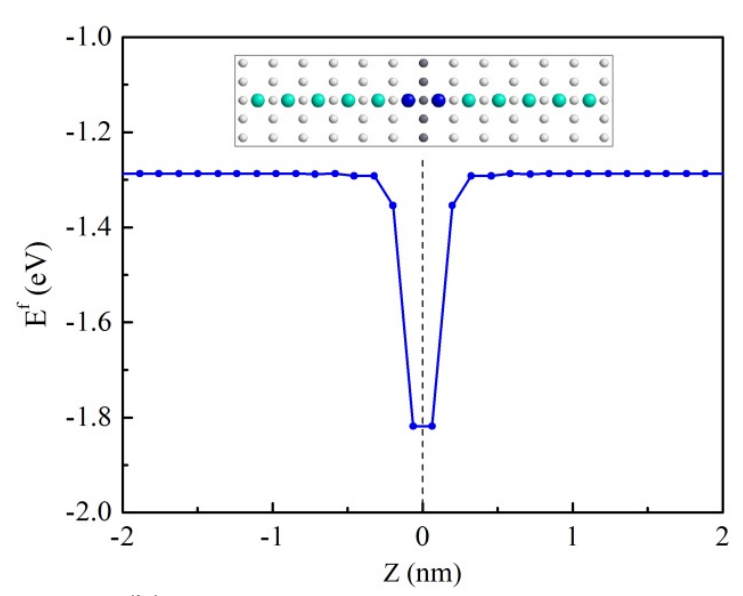

(b)

Figure 1. (a) Hydride formation energy as a function of H/Zr ratio, and (b) H segregation energy as a function of distance from the stacking fault. Larger atoms in the inset represent $\mathrm{H}$ colored by the segregation energy, and small ones represent $\mathrm{Zr}$, with the stacking fault denoted by dark dots.

The COMB potential gives good results on thermodynamical properties; however, it does not well capture the kinetics of $\mathrm{H}$ diffusion. Instead, we calculate the $\mathrm{H}$ diffusivity in $\alpha-\mathrm{Zr}$ using the reactive force field potential. In the calculation, one $\mathrm{H}$ atom is put into an octahedral interstitial site in $\alpha-Z r$. MD simulations are performed at a series of high temperatures between $1100 \mathrm{~K}$ and $1600 \mathrm{~K}$ and the mean-square displacements (MSDs) of the $\mathrm{H}$ are

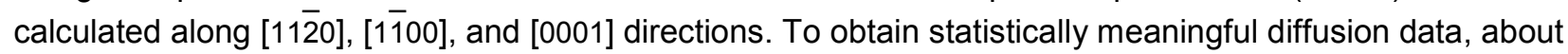
30 independent simulations are conducted at each temperature. In Figure 2a, the averaged MSD at $1500 \mathrm{~K}$ is shown. The linear fit of the MSD at each orientation gives the diffusivity at the corresponding orientation and temperature. By constructing the Arrhenius plot, which is the logarithmic diffusivity versus inverse temperature shown in Figure $2 \mathrm{~b}$, the prefactor and the activation barrier for $\mathrm{H}$ diffusivity are extracted. Our MD results give:

$D x=1.79 \times 10-6 \exp (-0.47 \mathrm{eV} / \mathrm{kBT}) \mathrm{m} 2 / \mathrm{s}$,

$D y=1.38 \times 10-6 \exp (-0.48 \mathrm{eV} / \mathrm{kBT}) \mathrm{m} 2 / \mathrm{s}$,

$D z=1.04 \times 10-6 \exp (-0.38 \mathrm{eV} / \mathrm{kBT}) \mathrm{m} 2 / \mathrm{s}$,

in excellent agreement with the averaged diffusivity measured from delayed hydride cracking experiments:

$D \exp =7.0 \times 10-7 \exp (-0.47 \mathrm{eV} / \mathrm{kBT}) \mathrm{m} 2 / \mathrm{s}$.

In addition, the activation barriers obtained from our MD simulations are in good agreement with DFT calculations. We also found that the diffusivity is slightly anisotropic with higher diffusivity along the $z$ ([0001]) direction. The orientation-dependent $\mathrm{H}$ diffusivity will be provided in upscale modeling in the future. More simulations are being conducted to improve the statistics of our calculations. 


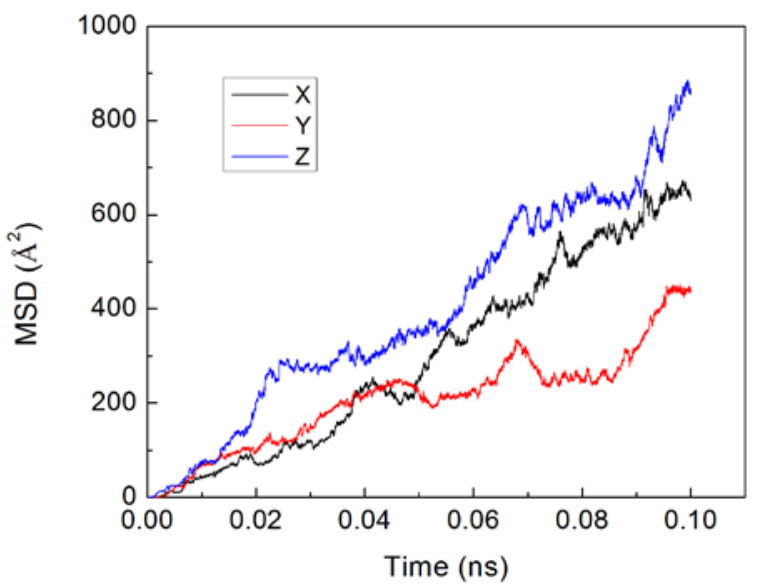

(a)

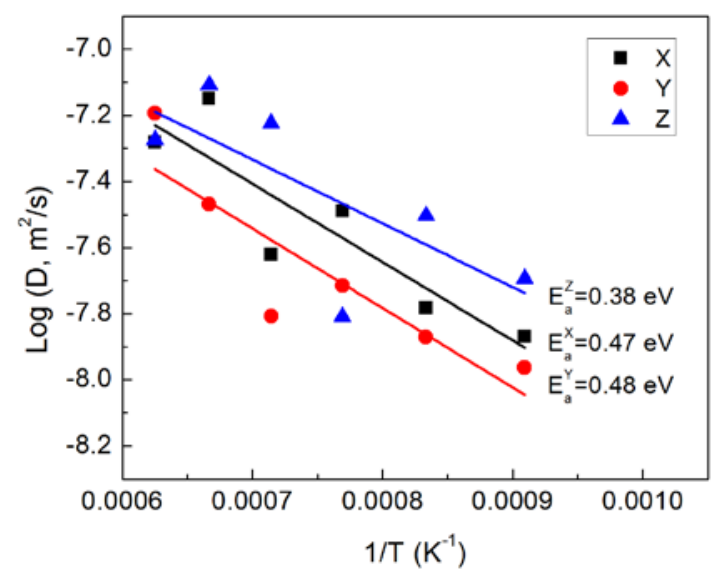

(b)

Figure 2. (a) Averaged MSD of an $\mathrm{H}$ atom at $1500 \mathrm{~K}$ and (b) the corresponding Arrhenius plots along X ([11 $\overline{2} 0])$, $Y([1 \overline{100]})$, and Z ([0001]) directions. The activation barriers agree well with both experiments and DFT calculations. The diffusivity is slightly anisotropic with higher diffusivity along Z direction.

\section{Benefits to DOE}

This project will improve the current understanding of hydride formation, in particular how the microstructure and stress state affect the $\mathrm{H}$ transport and the subsequent hydride nucleation in $\mathrm{Zr}$-based cladding. The success of the current work will help understand the effects of used fuel storage period extension on the environment. In turn it will help understand the risk in nuclear waste management in relevance to the mission of nuclear safety and energy security.

\section{Presentations}

Zhang, Y., X. Bai, M. Noordhoek, and S. Phillpot, "Molecular Dynamics Simulations of Zirconium/Zirconium-hydride Interface with the Charge Optimized Many Body (COMB) Potential," TMS 2015 Meeting, Orlando, FL, March 15-19, 2015.

Zhang, Y., W. Xu, P. Millett, and Y. Zhu, "Inter-facet Vacancy Diffusion and Void Nucleation in Hcp Metal," MRS 2014 Fall Meeting, Boston, MA, November 30-December 5, 2014. 


\title{
14-031 - Multidimensional Multiphysics Modeling of Fuel Behavior During Accident Conditions
}

\author{
Richard Williamson, Jason Hales, Giovanni Pastore, and Steve Novascone
}

Developing state-of-the-art computational tools for reliably predicting the behavior of nuclear reactor components during abnormal events is essential from both safety and economic standpoints. In particular, accurately assessing the thermo-mechanical behavior and integrity of the nuclear fuel rods (which comprise the core of current light water reactors) during accidents is of high importance for reactor safety analysis, as well as for design purposes. To this end, safety authorities and fuel designers rely heavily on dedicated fuel performance codes since they require minimal costs in comparison with the cost of experiments or an unexpected fuel rod failure. In addition, fuel performance codes able to cope with accident analysis are needed for the development of accident-tolerant fuel concepts, which has come to priority status at the DOE since the Fukushima Daiichi nuclear power plant accident in Japan in March 2011.

However, fuel performance code developments for accident analysis require a significant modeling and simulation effort. In particular, describing the global behavior of the fuel rods during accidents calls for the development of novel, advanced models for the numerous intricate and mutually dependent phenomena pertaining to both the fuel pellets and the surrounding metallic (Zircaloy) cladding tube. In fact, accident conditions bring on additional physics and increased complexity relative to normal reactor operation. For example, during a Loss-of-Coolant Accident (LOCA), as occurred at Three Mile Island in 1979 and at Fukushima, the Zircaloy fuel cladding experiences high temperatures while exposed to a low-pressure steam environment. The resulting, complex multiphysics response of the cladding includes rapid steam oxidation, solid-solid phase transition, and hydrogen $(\mathrm{H})$ uptake, which affect mechanical behavior; large plastic deformation, potentially leading to ballooning and bowing; thermal shock during a quench phase; and possible failure via bursting or melting. The fuel pellets also undergo fast temperature transients that lead to substantial, rapid release of fission gas to the pellet-cladding gap, contributing to cladding inner pressure increase and possible cladding failure via bursting. Current fuel performance codes attempt to approximate the inherently 3-D fuel rod behavior based on a 1-D radial approximation. Generally, separate codes are employed for normal operation and accident analysis, leading to difficulties with both code coupling and code management. Empirical models often do not exist for the high temperatures experienced during accidents, and uncoupled simulations are unrealistic during all operating conditions. It is important to replace these approaches with more predictive codes based on multidimensional, fully coupled multiphysics methods. INL's fuel performance code BISON meets these basic characteristics, offering a high potential for the improved computational analysis of fuel rod behavior during accidents.

Novel multiphysics models to describe nuclear fuel rod behavior during accidents were developed, implemented, and validated within the BISON code during this fiscal year. In particular, the research deals with advanced models for all main physical phenomena involved (i.e., transient fission gas behavior, rapid steam-cladding oxidation, Zircaloy solid-solid phase transition, $\mathrm{H}$ generation and transport through the cladding, and Zircaloy high-temperature non-linear mechanical behavior and failure).

The research is novel in that a fully coupled multidimensional multiphysics model of fuel and cladding behavior under high-temperature accident conditions has never been attempted. Such capability is not attainable with traditional fuel performance codes and will provide significantly improved accuracy and potentially improved numerical convergence. The proposed project represents a strong addition to the current nuclear fuel modeling 
efforts at INL and further demonstrates INL's developing excellence in modeling and simulation science. The work accomplished on this project in FY 2014 has raised the interest of other international institutes. Fruitful collaborations have been established and are planned to continue in the coming years. Specifically, collaborations on accident fuel behavior modeling are ongoing with the European Commission's Institute for Transuranium Elements (Germany) and the Politecnico di Milano (Italy). Such collaborations are enhancing the BISON development and validation processes for accident analysis through researchers' mobility between the involved institutes and through interaction with international experts. A joint INL-Institute for Transuranium Elements-Politecnico di Milano Ph.D. project has already been established, and the student has worked at INL for 6 months as an intern. This work has led to the successful incorporation and validation in BISON of a new fission gas release model applicable to accident conditions. The model and BISON results have been presented to an important international audience and received a broad consensus at the 2014 Enlarged Halden Programme Group Meeting in Røros, Norway. Also, as INL has enjoyed a long association with the Organization for Economic Cooperation and Development Halden Reactor Project, the present work will be closely coupled to experimental studies of accident behavior (such as LOCA testing) at the Halden Research Reactor. Furthermore, the BISON team will participate in the International Atomic Energy Agency sponsored Coordinated Research Project on Fuel Modeling under Accident Conditions. Participation in Fuel Modeling under Accident Conditions will involve a coordinated effort to test and advance accident analysis capability by comparison to experimental data and other fuel performance codes, also providing INL access to high-quality experimental data.

\section{Summary}

Key FY 2014 accomplishments include:

- Development and validation of a new fission gas behavior model. This model permits to reproduce rapid gas release during accident transients based on a description of the underlying physical processes and has been shown to reliably capture experimental behavior.

- Implementation and testing of models for steam-cladding oxidation during accident conditions, also accounting for the related effects on the fuel rod thermal performance.

- Development and validation of interactive models for high-temperature creep and solid-solid phase transition of Zircaloy, important for predicting cladding mechanical behavior including failure.

- Development and preliminary validation of a model to predict $\mathrm{H}$ diffusion and precipitation in Zircaloy cladding, also important for predicting cladding mechanical behavior and potential failure.

- Demonstration of accident capabilities for a fuel rod undergoing a LOCA accident, including rapid fuel rod heating leading to extensive cladding ballooning. An example of BISON results is illustrated in Figure 1, showing the calculated temperature distribution in the fuel rod during a LOCA. Cladding ballooning as suitably reproduced by BISON is also evident. 


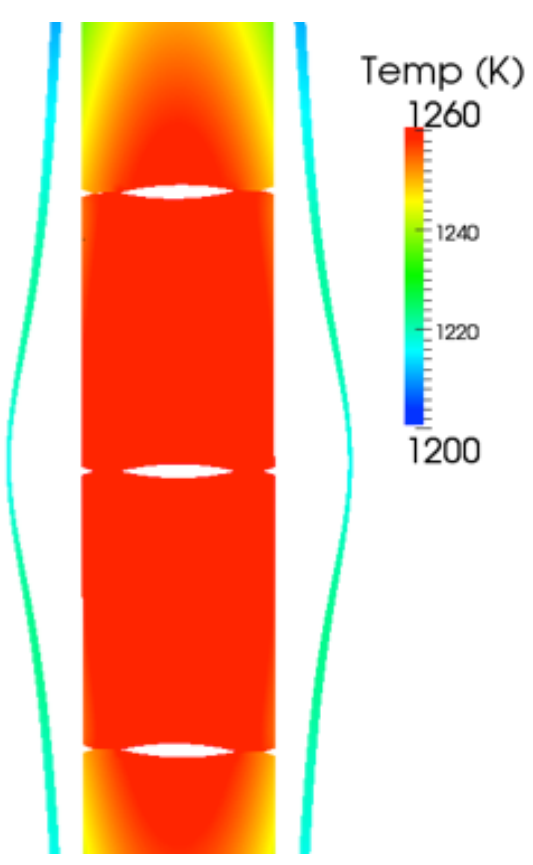

Figure 1. Example of BISON results for a fuel rod behavior simulation during a LOCA accident.

\section{Benefits to DOE}

This project will directly benefit the nuclear energy mission of the DOE by addressing one of today's most critical nuclear energy concerns, design basis accidents. Given the experimental evidence from programs such as the Halden Reactor Project, understanding LOCAs and reducing their likelihood are key concerns. This project will leverage the unique capabilities of BISON and result in the ability to explore accident behavior in a novel way with fully coupled solutions to the 3-D problem.

\section{Publications}

Pastore, G., D. Pizzocri, J. Hales, S. Novascone, D. Perez, B. Spencer, R. Williamson, P. Van Uffelen, and L. Luzzi, "Modelling of Transient Fission Gas Behaviour in Oxide Fuel and Application to the BISON Code," Proceedings of the Enlarged Halden Programme Group Meeting, Røros, Norway, September 7-12, 2014.

Chakraborty, P., M. Tonks, and G. Pastore, "Modeling the Influence of Bubble Pressure on Grain Boundary Separation and Fission Gas Release," J. Nucl. Mater., Vol. 452, 95-101, 2014.

\section{Presentations}

Williamson, R., G. Pastore, S. Novascone, J. Hales, B. Spencer, and D. Perez, "Modeling LOCA Behavior with the BISON Fuel Performance Code," 20th QUENCH Workshop, Karlsruhe, Germany, November 11-13, 2014.

Pastore, G., D. Pizzocri, J. Hales, S. Novascone, D. Perez, B. Spencer, R. Williamson, P. Van Uffelen, and L. Luzzi, "Modelling of Transient Fission Gas Behaviour in Oxide Fuel and Application to the BISON Code," Enlarged Halden Programme Group Meeting, Røros, Norway, September 7-12, 2014. 


\title{
14-038 - Advanced Automated Validation of Modeling and Simulation Tools
}

\author{
Curtis Smith, Robert Youngblood, Cristian Rabiti, Doug Burns, and Jacopo Buongiorno
}

Large-scale or complex codes for simulation of facility safety may contain parameters or models that are not known precisely - this limits confidence in the simulation results. To be able to use the results of these simulation codes with confidence, it is important to learn as much as we can about the behavior of these codes. Information from tests (i.e., experiments, both old and new) or operating experience has typically been incorporated into safety codes by a process known as "calibration," which reduces uncertainty in the output of the safety code and thereby improves its support for decision-making.

Modern analysis capabilities allow for significant improvements on classical ways of doing both calibration and validation. Recently, key innovations have come from the development of code surrogate approaches (emulators) and Bayesian approaches.

The objective of this LDRD is to develop a validation and quantification approach that will achieve the following:

1. Compare, via a probabilistic method, experimental data with simulation model results using a computerized and automated approach

2. Approach validation from a probabilistic perspective for comparison (as compared to traditional qualitative comparisons)

3. Apply a quantitative method that focuses on those parts of simulation model outputs that are more important (for example, steady state is less important than transient behavior)

4. Use emulators to assist in the model assessment

5. Provide storage of experimental data to automate retrieval for quantification

Clone Fulkushima

Information Portal for use

as a Validation

Information Portal (VIP)
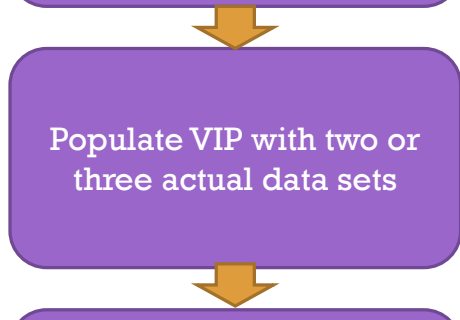

Extend VIP to allow for

extraction of validation

data (via a programmable interface)

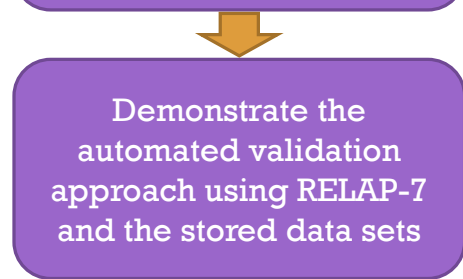

Gaussian Process Models (GPM) based upon MIT work

Bayesian Code Validation

6. Be automated to determine acceptance of the validation results for a specific simulation model.

These steps are illustrated in the figure above. 


\section{Summary}

The R\&D progress this year has been centered on three integrated activities:

First, we have transitioned a cloned version of the INL-developed Fukushima Information Portal as a starting point for the validation database infrastructure. This data structure provides the backbone for both the data storage (e.g., experimental data sources) and the validation of numerical approaches.

Second, we have investigated several sources of experimental data that would be applicable for validation approaches against thermal-hydraulic codes (e.g., RELAP) we are considering. An example of these data sources includes the boiling water reactor Full Integral Simulation Test reports (and associated experimental results) that are available through the Electric Power Research Institute.

Third, with Massachusetts Institute of Technology, we have looked into how to extend assessment to non-trivial numerical approaches of validation. We have worked with Gaussian Process Models that make use of Monte Carlo Markov Chain methods. This approach builds upon the recent Massachusetts Institute of Technology Ph.D. work of Dr. Joseph Yurko, who was supported on this project (successful defense of his doctoral thesis in FY 2014). One of the outcomes of this work was the development of an emulator construction approach representing complex phenomena (such as plant thermal-hydraulics) that are very fast running to the point that computational time is negligible in comparison to creating the original computer simulator training set. Demonstrated enhancements in speed-up computational time were on the order of 300-700 times. For example, a calculation that takes 500 seconds (almost $81 / 2$ minutes) using a code like RELAP would take about a second using the emulator.

To date, data management capabilities have been added to the RAVEN code. In particular, the focus has been on the creation of a reader capable of converting comma-separated value files to the internal RAVEN data storage system. This will allow using the statistical capabilities of the RAVEN code to perform comparison between experimental data and simulation data. Further, we have completed a requirements document, focusing on the needed statistical support from RAVEN as part of the automated validation that has been proposed.

\section{Benefits to DOE}

DOE-NE'S mission relies essentially on using large-scale simulations to support decision-making for both safety and operational purposes. For example, deployment of new reactor technologies (such as small modular reactors) will need a new simulation capability. This project will lead to a significant state-of-the-art improvement in calibrating these simulations and validating codes so that they better predict reality. The techniques will benefit any technology making use of system-scale simulations in its decision-making, such as the National Aeronautics and Space Administration.

\section{Presentations}

Youngblood, R., "Application of Gaussian Process Modeling to Analysis of Functional Unreliability," Proceedings of PSAM-12 (Probabilistic Safety Assessment and Management), June 22-27, 2014. 


\section{4-041 - Fabrication of $\mathrm{UN}-\mathrm{U}_{3} \mathrm{Si}_{2}$ Composites via Spark Plasma Sintering}

Paul A. Lessing, Rob O’Brien, and Jason Harp

The goal for the work performed during FY 2014 was to examine the feasibility of fabricating uranium mononitride (UN)-uranium disilicide $\left(\mathrm{U}_{3} \mathrm{Si}_{2}\right)$ composites using the Spark Plasma Sintering (SPS) process. The specifically desired microstructure was to create microencapsulation of UN particles by amorphous $U_{3} \mathrm{Si}_{2}$. A volume fraction of $60 \% \mathrm{UN}$ in a $40 \% \mathrm{U}_{3} \mathrm{Si}_{2}$ matrix was examined to provide a sufficient uranium $(\mathrm{U})$ atom density in the composite such that these compacts may be used as a replacement for $\mathrm{UO}_{2}$ fuel pellets in existing and future power reactors.

\section{Summary}

The SPS process utilizes rapid pulses of direct current at voltages of up to $10 \mathrm{~V}$ and typical current densities of order $103 \mathrm{~A} / \mathrm{cm}^{2}$ to $104 \mathrm{~A} / \mathrm{cm}^{2}$ to sinter powdered materials held within a conductive die. The sintering mechanisms that take place during SPS sintering are highly dependent upon the electrical properties of the materials being processed. For electrically conductive materials, the process promotes initial rapid joule heating at high resistance points across the microstructure. For non-conductive materials, the rapid joule heating of the surrounding die results in rapid heating of the powdered materials enclosed via thermal conduction, which in turn promotes grain boundary diffusion and surface diffusion.

The preliminary results have successfully demonstrated the feasibility of producing sintered composites of $\mathrm{UN}-\mathrm{U}_{3} \mathrm{Si}_{2}$ to high density via the SPS process. The work has identified a peak sintering temperature of $1,300^{\circ} \mathrm{C}$ and has determined minor challenges that can be solved through the control of cooling rates in future work. Microstructure evidence conclusively shows that the $U N-U_{3} \mathrm{Si}_{2}$ composite was densified by liquid phase sintering where the $U_{3} \mathrm{Si}_{2}$ phase surrounds every grain of $U N$. This is illustrated in Figures 1 and 2 . The more corrosion-resistant phase $\left(\mathrm{U}_{3} \mathrm{Si}_{2}\right)$ has melted and completely surrounded each of the $\mathrm{UN}$ grains. Liquid phase sintering enhancement requires a molten second phase that wets and spreads on powder surfaces within a compact. Figures 1 and 2 show clear evidence that all four stages of liquid phase sintering (i.e., wetting, rearrangement, dissolution-re-precipitation, and agglomeration) have occurred.

\section{Benefits to DOE}

Fuel pellets composed of a composite matrix of $\mathrm{U}_{3} \mathrm{Si}_{2}$ and $\mathrm{UN}$ are of interest as an accident tolerant high-density $\mathrm{U}$ dispersion fuel-form for light water reactors. Specifically, these pellets might be a direct substitution for metallic fuels or $\mathrm{UO}_{2}$ fuels in the existing light water reactor fleet. Also, the improved thermal conductivity of the $\mathrm{U}_{3} \mathrm{Si}_{2}-\mathrm{UN}$ fuel increases the safety margin of the reactor. 


\section{NUCLEAR SCIENCE \& TECHNOLOGY}

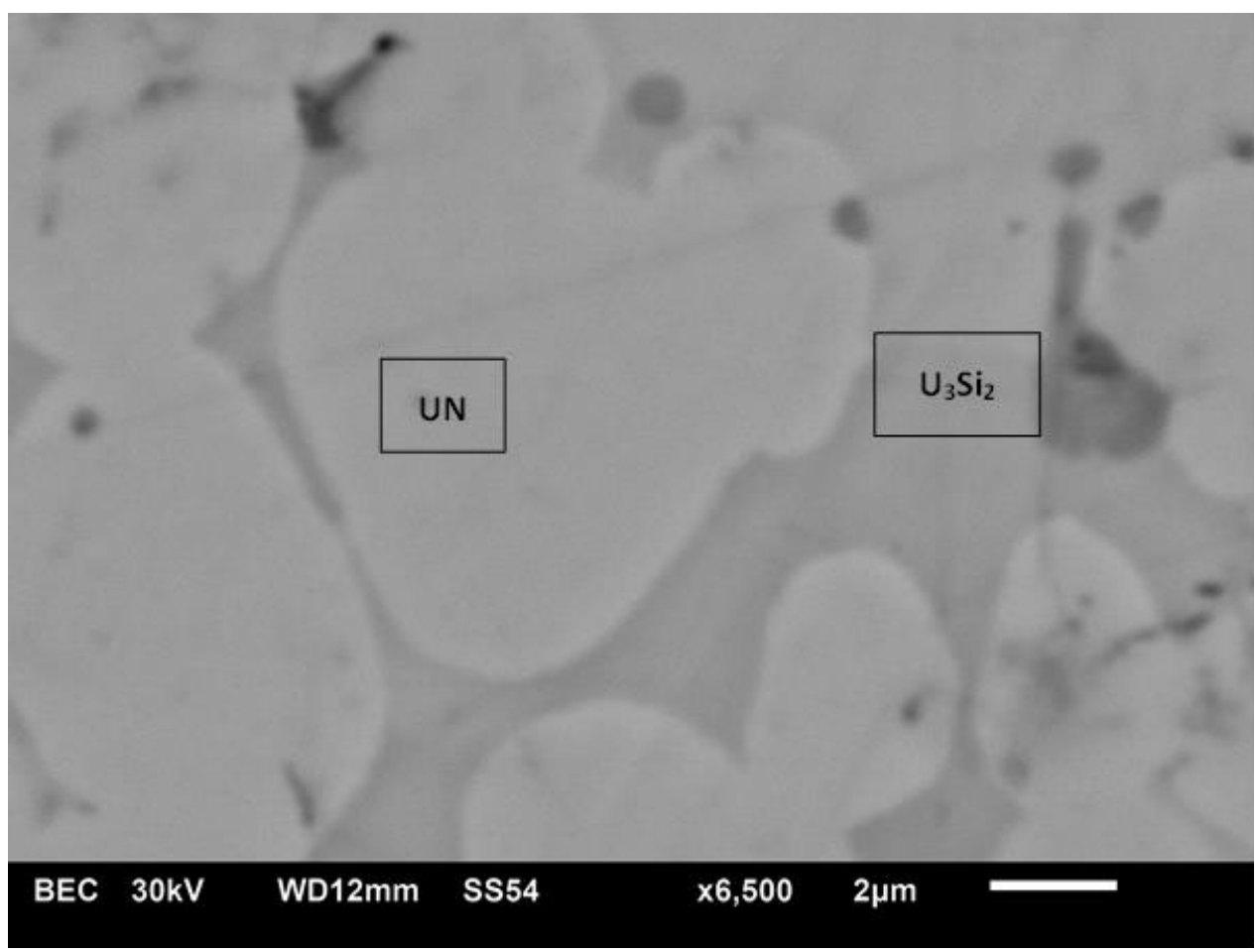

Figure 1. Scanning Electron Microscope Backscatter Electron image of a UN- $U_{3} \mathrm{Si}_{2}$ composite sample using SPS.

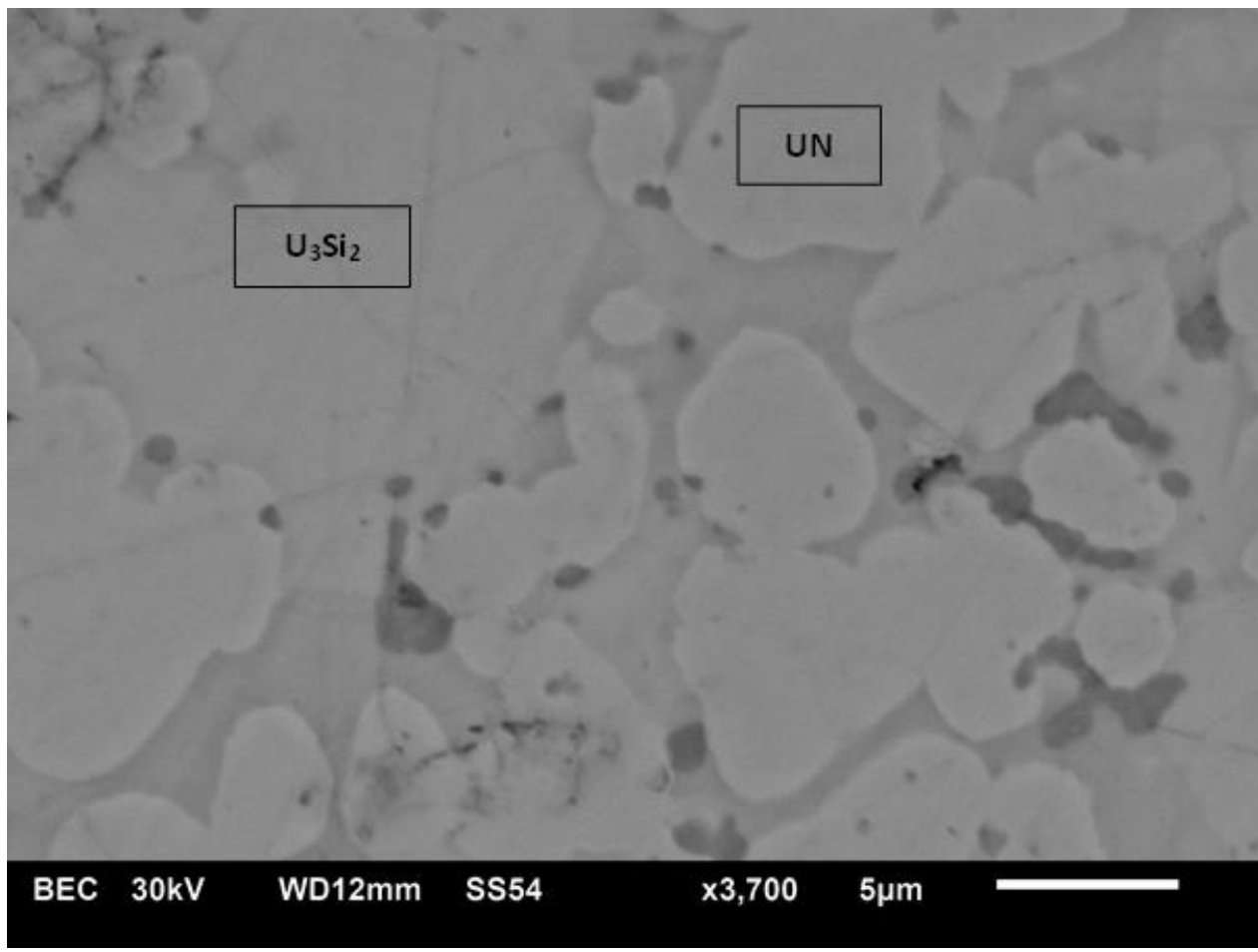

Figure 2. Scanning Electron Microscope Backscatter Electron image of a UN- $U_{3} \mathrm{Si}_{2}$ composite sample produced using SPS. 


\title{
14-075 - Development of Tools and Methodologies for Uncertainty Quantification and Validation for Multiphysics Fuel Performance Simulation
}

\author{
Cristian Rabiti, Richard Williamson, and Anil Prinja ${ }^{1}$
}

Fuel performance analysis is characterized by a long computational time further complicated by generally highly non-linear phenomena. Many of the parameters used in the fuel behavior models are characterized by large uncertainties. Multi-physics simulations bring in additional uncertainties from other models not used in typical fuel modeling. In particular, coupling neutronics calculations with fuel performance calculations exacerbates this behavior by introducing additional non-linearity and increasing the dimensionality of the input space. Given these complexities introduced by sophisticated modeling, the estimation of uncertainties characterizing fuel performance simulation is a difficult challenge and the goal of this LDRD project.

Fortunately there are only a few figures of merit that are of interest to engineers. Therefore, the effective dimensionality of the input space is smaller than the total problem dimensionality. In other words, the fact that engineering decisions are made relying on a few figures of merit implies the existence of linear combinations of variations of the input parameters with respect to which the figures of merit are insensitive.

These considerations suggest that reduced order models could be positively used to replace real simulations and to propagate uncertainties. When the effective dimensionality is low, it is possible to construct mathematical models (reduced order models) that are of a fast evaluation and replace the original expensive models. These reduced order models could be used to analyze the propagation of uncertainty with a very low computational time.

Part of the work covered by the LDRD is assessing which reduced order models are most suitable for this task, how to make such determinations, and evaluating when a reduced order model has received enough training to replace the real model in the uncertainty quantification.

Another direction of the current work sponsored by this LDRD is the application of sparse grid sampling for the evaluation of the uncertainty affecting the nuclear cross section. Sparse grid samplings are effective methodologies to reduce the sampling of the input space necessary to construct a truthful representation of the response of the system to variation of the input parameters (i.e., cross sections). These methodologies are even more effective when combined with techniques that selectively implement a heterogeneous interpolating order, like analysis of variance.

\section{Summary}

The coupling between RAVEN and BISON has been completed. The characterization of the dimensionality and non-linearity of a prototypical fuel performance analysis has been started. Part of the BISON reference analysis is still ongoing. The complete analysis will be found in the paper submitted to the International Topical Meeting on Probabilistic Safety Assessment and Analysis 2015 entitled "Fuel Reliability Analysis Using BISON and RAVEN."

\footnotetext{
${ }^{1}$ University of New Mexico
} 
The test chosen for the BISON runs is described in "Multidimensional Multiphysics Simulation of Nuclear Fuel behavior" by R. Williamson, J. Hales, S. Novascone, M. Tonks, D. Gaston, C. Permann, D. Andrs, and R. Martineau. The parametric dependence analysis is performed with respect to these parameters:

- Power scaling factor

- Thermal expansion coefficient

- Grain radius scaling factor.

Each BISON run takes a few hours, and several different sampling strategies have been tested and are currently running. The number of sampling points (runs of the BISON code corresponding to a specific point in the input space) for each test performed is on the order of 8000 to ensure a good statistical convergence. Figures 1-3 show a 4-D plot where the chosen figure of merit (maximum over time and average over space, hoop stress in the clad) is plotted as a function of the other input variables. The plots reveal limited non-linear effects that could be inferred both from the general curvature and by the variation in the thickness of the surfaces. The magnitude of non-linearity is an indicator of the complexity that a surrogate model is approximating and will have to be addressed, making this BISON replacement more challenging.

Several supervised algorithms (also known as surrogate models) have been implemented and tested to construct a surrogate of the BISON simulation. A deeper analysis of the quality of those surrogates will be performed next year.
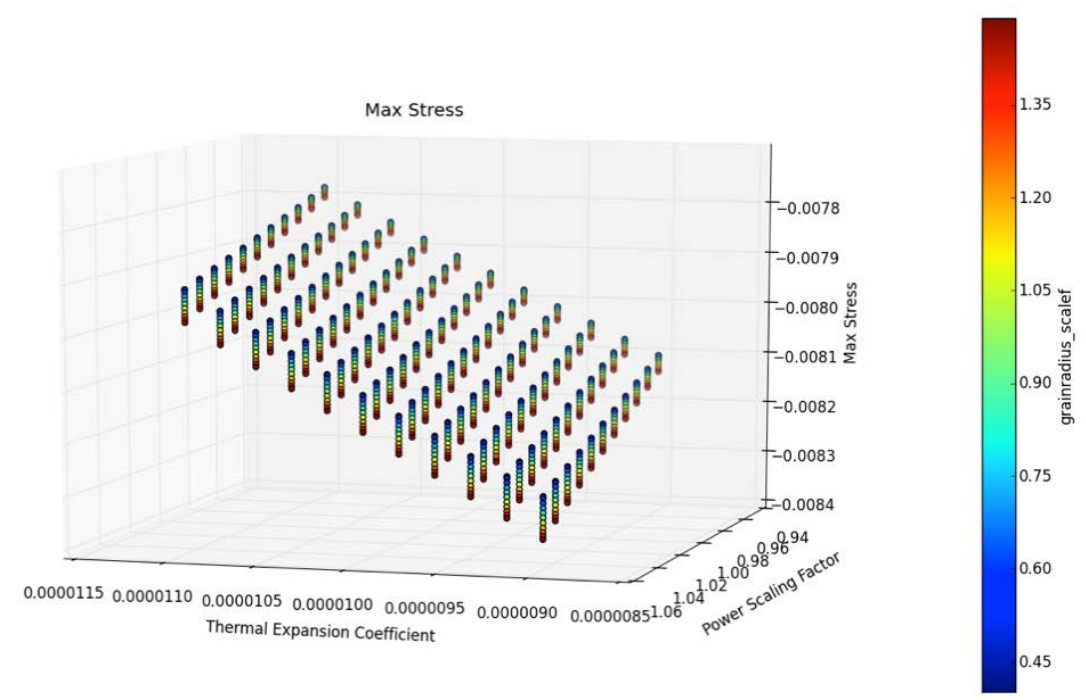

Figure 1. Maximum (over time) average hoop stress in the clad. 


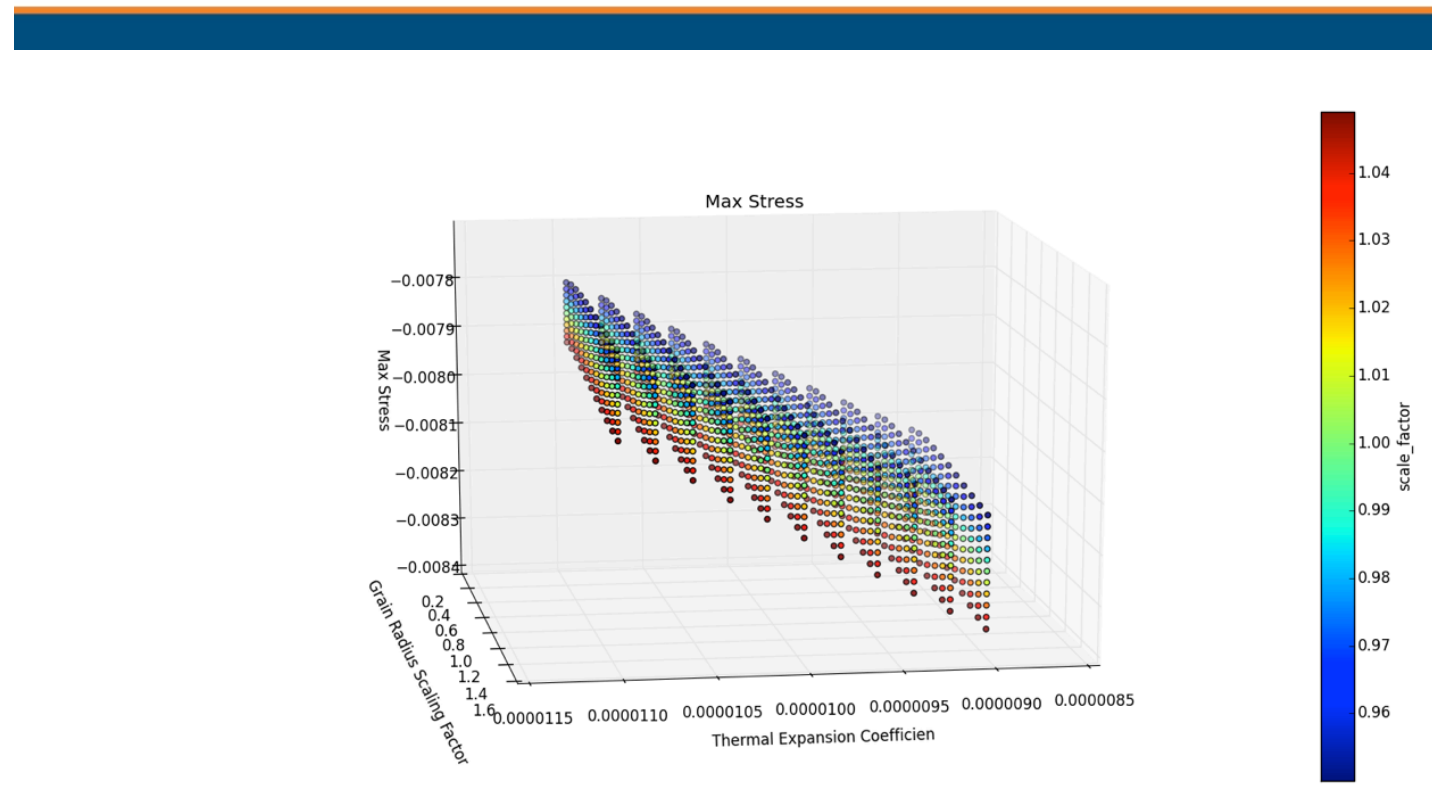

Figure 2. Maximum (over time) average hoop stress in the clad.
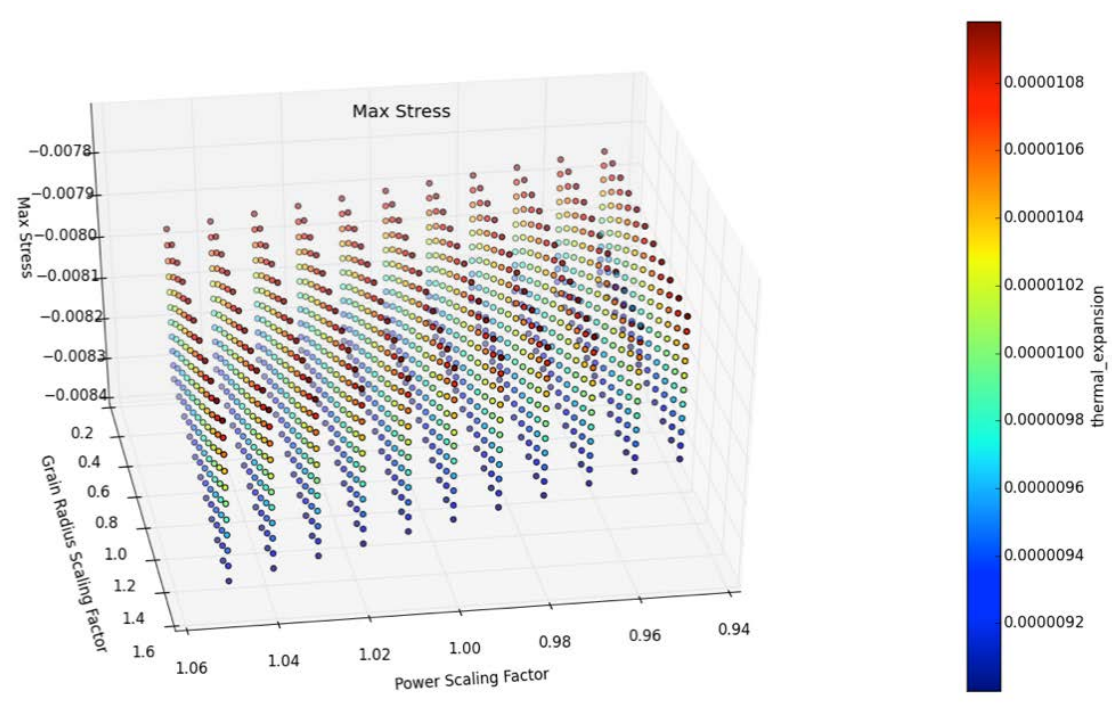

Figure 3. Maximum (over time) average hoop stress in the clad.

Figures 4 and 5 show the results of different Monte Carlo sampling schemes applied directly to the supervised learning algorithm that approximates the BISON code. These figures are an illustration of the possible usage of surrogate models. The surrogate model is sampled using two different probabilistic distributions for the grain-scaling factor, allowing the analysis of the propagation of uncertainty under different assumptions of the initial uncertainty affecting this parameter. This could be done without running BISON for the two different cases by using the surrogate model instead, resulting in a large computational time saving. 
The effect of the two different distributions used to model the grain radius scaling factor can be observed by the different clustering of the points with respect to this variable. For example, Figure 4 reflects a lower value of the sigma, leading to a more clustered set of points.

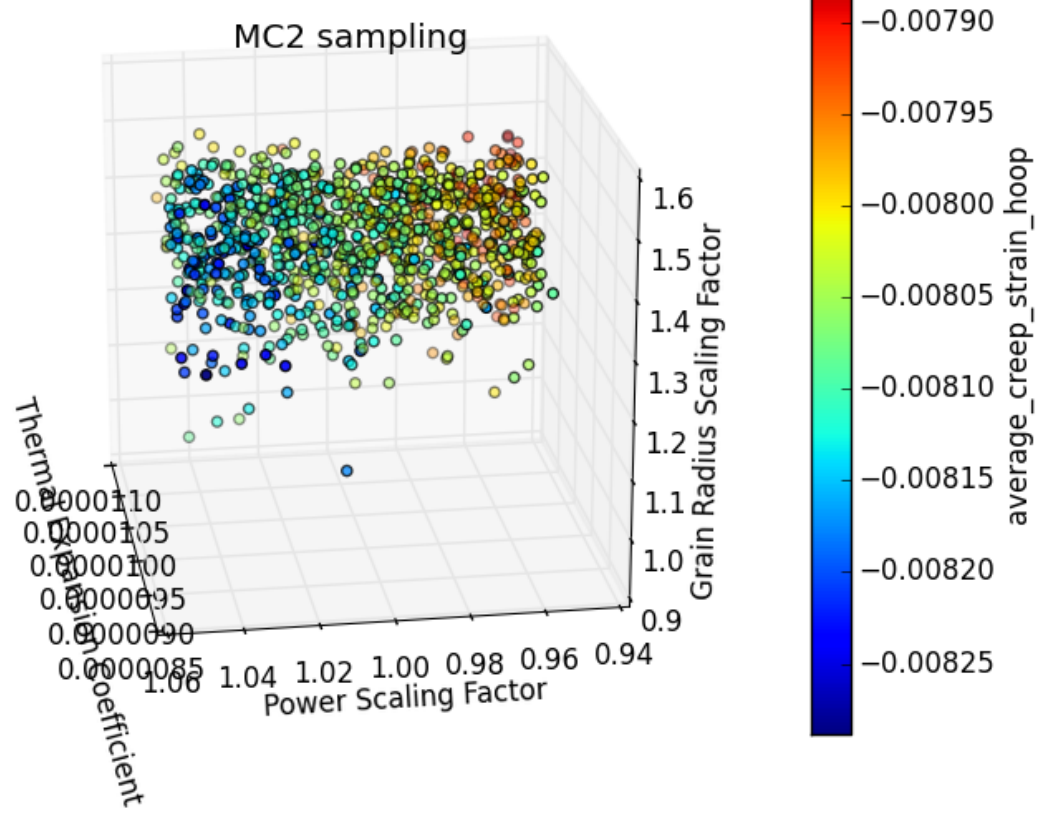

Figure 4. Sampling with truncated distribution on the grain radius scaling factor at 1.5.

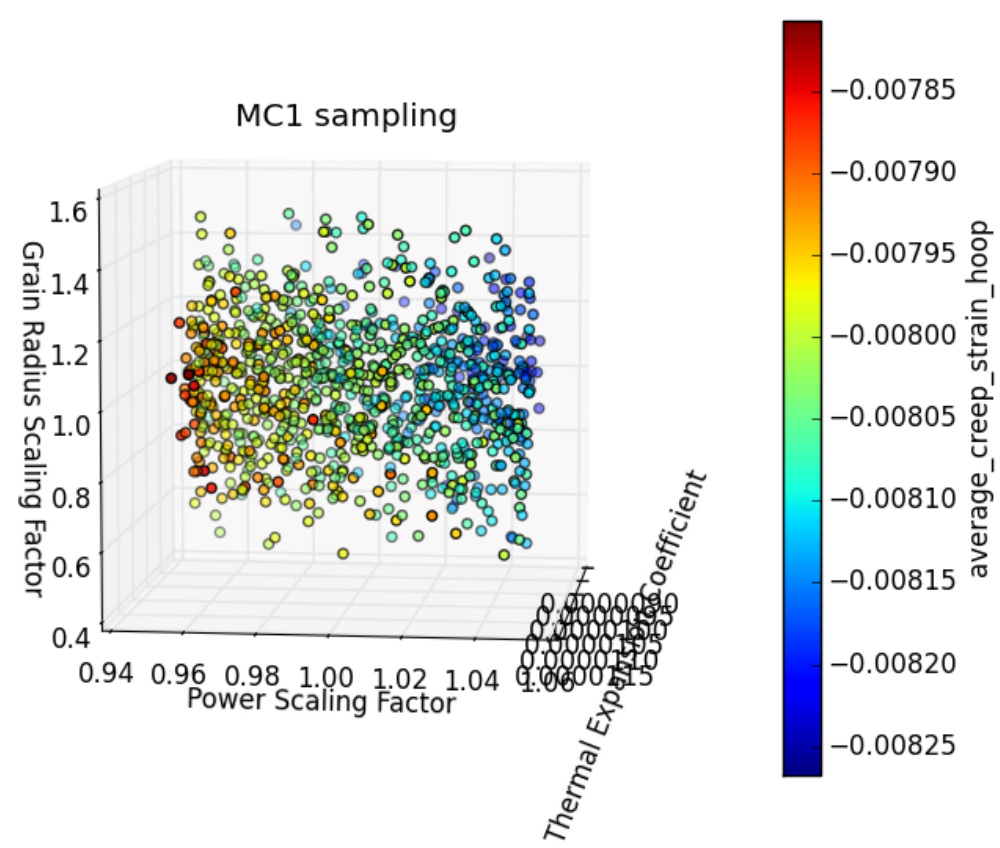

Figure 5. Sampling without truncated distribution on the grain radius scaling factor. 
Comparison between uncertainty propagation results obtained using the BISON code and its surrogates will be performed soon to confirm the accuracy of the surrogate models for uncertainty analysis.

Next year, the possibility of developing algorithms capable of automatically assessing the quality of the surrogate model generated (active learning) will be explored.

Table 1 reports the classical parameters used in uncertainty quantification to assess the quality of the simulation with respect to the figures of merit. In this case, the uncertainty on the chosen figure of merit (maximum over time and average over space, hoop stress in the clad) are computed from the 8000 Monte Carlo runs using the original BISON model, and in the future they will be compared with the results obtained by the usage of a surrogate model.

Table 1. Statistical parameters characterizing the maximum (time) average (space) hoop stress for the unbounded Monte Carlo sampling.

\begin{tabular}{|l|l|l|l|}
\hline \multicolumn{1}{|c|}{ Quantity: } & \multicolumn{1}{|c|}{ Value } & \multicolumn{1}{c|}{ Quantity: } & \multicolumn{1}{c|}{ Value } \\
\hline Median & $-8.03733000 \mathrm{E}-03$ & kurtois & $-2.97078524 \mathrm{E}-01$ \\
\hline Mean & $-8.04134073 \mathrm{E}-03$ & Percentile 95\% & $-7.92044493 \mathrm{E}-03$ \\
\hline Variance & $7.68609489 \mathrm{E}-05$ & skewness & $-1.00483713 \mathrm{E}-01$ \\
\hline
\end{tabular}

A Ph.D. student at the University of New Mexico has started testing sparse grid methodologies on a homegrown, basic neutron diffusion code. The results are promising and reported in a paper entitled "Sparse-Grid Stochastic Collocation Uncertainty Quantification Convergence for Multigroup Diffusion," which has been accepted at the next American Nuclear Society winter meeting. It is foreseeable that the student will spend part of the next FY at INL implementing the methodology in RAVEN.

\section{Benefits to DOE}

Benefits from the introduction of high fidelity simulation tools have been experienced in many other fields, and in the last 10 years DOE has invested in the creation of such tools for nuclear energy application. The nuclear sector is highly regulated, and as a consequence it exhibits a strong inertia toward adoption of new technologies. It is clear that without a strong quantification of a new tool's uncertainties, it will not be allowed to be licensed in the nuclear industry. This is exactly the point addressed by this proposal, and success will allow the industrial deployment of several million dollars of already invested money, generating a true benefit to the nuclear industry.

\section{Publications}

Rabiti, C., J. Cogliati, G. Pastore, and A. Alfonsi, "Fuel Reliability Analysis Using BISON and RAVEN," International Topical Meeting on Probabilistic Safety Assessment and Analysis, Sun Valley, ID, April 26-April 30, 2015, accepted.

Talbot, P., and A. Prinja, "Sparse Grid Stochastic Collocation Uncertainty Quantification Convergence for Multigroup Diffusion," Proceedings of the 2014 ANS Winter Meeting, Anaheim, CA, November 9-13, 2014. 


\section{4-098 — Irradiation Effects in Uranium Dioxide}

Jian Gan, Kumar Sridharan ${ }^{1}$, Beata Tyburska-Püschel, ${ }^{1}$ and Jeff Terry $^{2}$

The evolution of microstructure and properties of ceramic nuclear fuel $\mathrm{UO}_{2}$ during neutron irradiation is crucial to the current and future nuclear power plants operating globally. Despite large existing empirical databases for $\mathrm{UO}_{2}$, there is a lack of scientific understanding of the defect progression in this material under irradiation. The point defect growth and clustering in neutron and ion irradiated depleted $\mathrm{UO}_{2}$ is being studied by advanced synchrotron-based micro Extended X-ray Absorption Fine Structure ( $\mu$-EXAFS) methods and transmission electron microscopy. EXAFS has already been shown to be a powerful tool for characterizing local order in off-stoichiometric $\mathrm{UO}_{2}$, but thus far it lacked spatial resolution. In this research, the novel spatially resolved $\mu$-EXAFS has been used for characterizing irradiation damage in $\mathrm{UO}_{2}$. Small sample size (focused ion beam [FIB] lamella) allows examination of highly activated materials, and the small $x$-ray spot size allows damage profiling as a function of depth. Combining the $\mu$-EXAFS with ion irradiation allows multiple damage levels to be examined within the same sample and a direct comparison with transmission electron microscopy analysis. Simulation, modeling, and data fitting are a significant part of understanding the measured EXAFS spectra.

\section{Summary}

The EXAFS analysis has shown that ion-irradiation disrupts local structure by giving rise to multisite oxygen (O) distribution at approximately $1.9 \AA$ from the absorbing atom $\left(0.2 \AA\right.$ longer than in documented $\left.\cup O_{2+x}\right)$, similar to $\mathrm{O}$ interstitials created in $\mathrm{UO}_{2+x}$; however, there is no known oxidation of the material. The multisite distribution results from uranyl type bonds that are $\sim 1.8 \AA$ in length and highly stable due to their oblate geometry. Therefore, they distort the original local structure. The EXAFS data of the irradiated $\mathrm{d}-\mathrm{UO}_{2}$ show that an increasing irradiation dose results in a consistent decrease in amplitude of the crystallographic shells, longer bond distances between near neighbors, and the appearance and increase of a shoulder on the low $R$ (at $1.9 \AA$ ) side of the first crystallographic U-O shell. Random disorder is not evident in this set of samples according to the processed EXAFS characteristic. Due to the consistent amplitude reduction as shown in Figure 1, it can be theorized that the defects are not random but instead are distributed such that the overall lattice retains its $\mathrm{UO}_{2}$ structure even at higher irradiation doses. In other words, the defects do not modify the cubic $\mathrm{UO}_{2}$ structure. This is consistent with several studies that indicate that fluorite ceramics in a reactor maintain their lattice structure even at high irradiation doses.

\footnotetext{
${ }^{1}$ University of Wisconsin

${ }^{2}$ Illinois Institute of Technology
} 

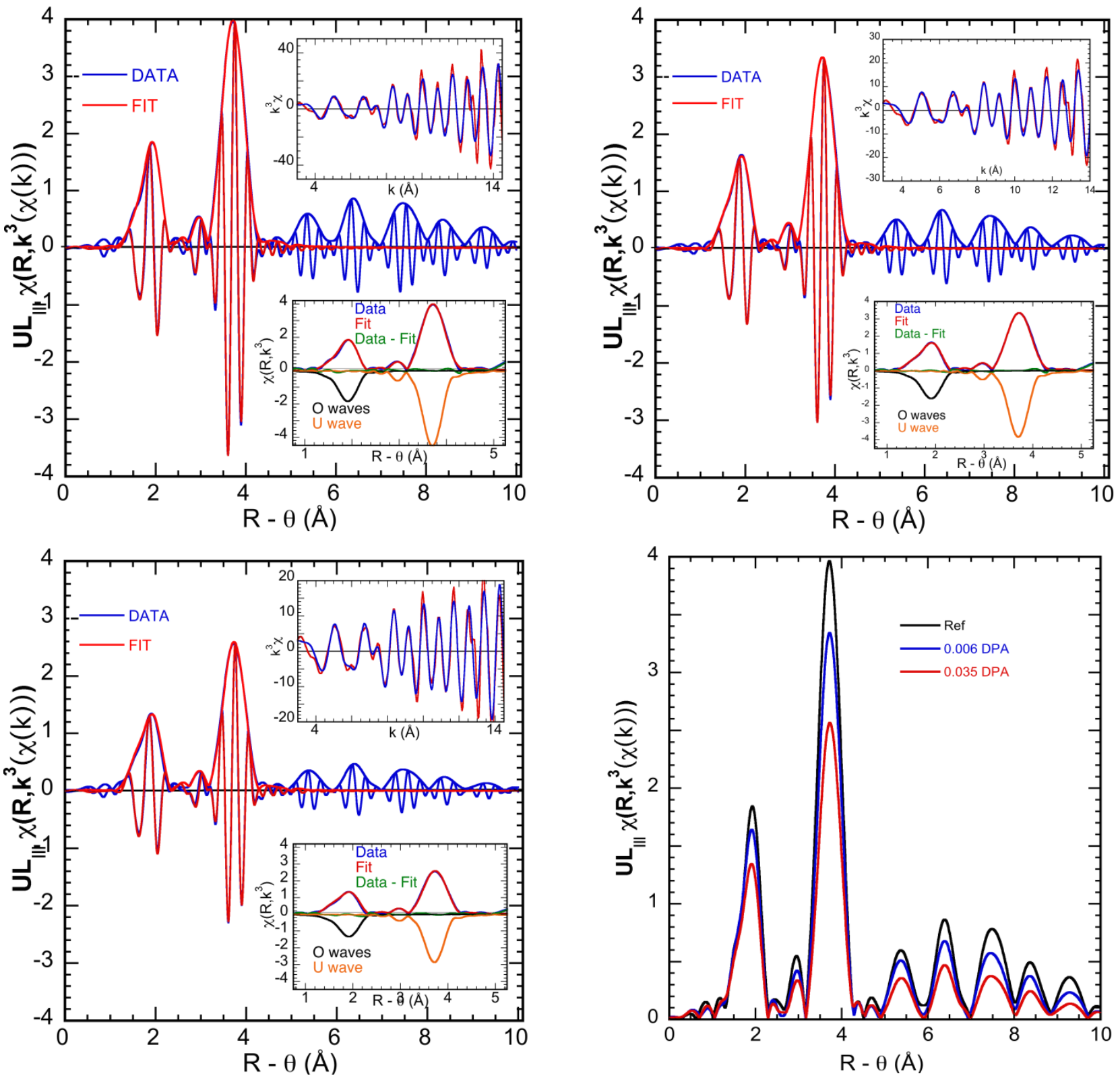

Figure 1. The $k^{3} \chi(R)$ EXAFS of reference $\mathrm{UO}_{2}\left(\mathrm{UO}_{2}\right.$ irradiated with $\mathrm{He}^{2+}$ ions to 0.006 and $\left.0.035 \mathrm{dpa}\right)$. The modulus of the real part of the transform of both data and fit are shown in the plots 1-3. The insets show the $k^{3}$ $\chi$ spectra overlaid with curve-fit (top) and the moduli (bottom) of the data, fit, difference between data and fit, and the individual contributions to the fit (inverted for clarity). Plot 4 shows the modulus of the Fourier transforms of the $k^{3}$ - weighted EXAFS spectra of indicated samples. Transforms were performed over range $2.70-14.75 \AA^{-1}$. 
All LDRD milestones have been met with successful development of technique to perform $\mu$-EXAFS measurements at the Stanford Synchrotron Radiation Lab. A FIB instrument was used to extract FIB lamella consisting of the damage layer created by helium $(\mathrm{He})$ ions implanted into bulk $\mathrm{UO}_{2}$ samples. Five such lamellae, consisting of two each from the two irradiated samples and one from the pristine sample, were mounted onto a FIB grid, which was then inserted into a $\mu$-EXAFS holder specially designed for the measurements at the Stanford Synchrotron Radiation Lab. These samples were measured at a $45^{\circ}$ angle from the incident beam in fluorescence geometry. Further, bulk EXAFS analysis was performed on krypton $(\mathrm{Kr})$ implanted samples at a $10^{\circ}$ angle from the incident beam to interrogate the irradiated region of depth $1.1 \mu \mathrm{m}$. He implanted samples (0.006-0.035 dpa) and proton irradiated samples $(0.01-0.5 \mathrm{dpa}$ ) were also successfully studied using EXAFS at the Stanford Synchrotron Radiation Lab.

\section{Benefits to DOE}

The investigation of an irradiated $\mathrm{UO}_{2}$ microstructure using both transmission electron microscopy and EXAFS/ $\mu$-EXAFS provides a unique opportunity to connect the lattice disorder and defect clustering to the molecular dynamics modeling simulation. INL has benefited from this work by further enhancing its R\&D strength on fuel material microstructure characterization and modeling. Our work has contributed to DOE's leading role on basic science research of materials behavior and performance in extreme environments. Being able to access a different facility in a different institution is critical to the success of this project. Further, with the progress of the extended fuel storage option for used nuclear fuel, this research directly facilitates the understanding of the stability of $\mathrm{UO}_{2}$ fuels following irradiation.

\section{Publications}

Gupta, M., S. Conradson, J. Pakarinen, and T. Allen, "Local Structure Analysis of He2+ irradiated UO2 using Extended X-ray Absorption Fine Structure," J. Appl. Phys., November 2014, planned submission.

Gupta, M., S. Conradson, L. He, J. Pakarinen, and T. Allen, "Lattice Structure Evolution in Krypton Irradiated UO2: A TEM and XAFS Study," J. Appl. Phys. or J. Nucl. Mater., December 2014, planned submission.

\section{Presentations}

Gupta, M., "Defects in Ion Irradiated $\mathrm{UO}_{2}$ on Multiple Length Scales: A TEM and XAFS Study," Presentation given to Dr. Ernest Moniz (U.S. Secretary of Energy), August 19, 2014.

Gupta, M., "Damage Structure Evolution in Ion Irradiated $\mathrm{UO}_{2}$," Presentation Given to Dr. Patricia Dehmer (Deputy Director, U.S. DOE Office of Science), August 12, 2014.

Gupta, M., "Damage Structure Evolution in Ion Irradiated $\mathrm{UO}_{2}$," TMS Conference 2014, San Diego, CA, February 18, 2014. 


\section{4-102 - Advanced Seismic Probabilistic Risk Assessments}

\section{Justin Coleman}

This LDRD project was funded in FY 2014 and continued for the first 6 months of FY 2014. At that time, the LDRD was converted to a direct funded project. This report covers the objectives and purpose of performing advanced seismic probabilistic risk assessments (SPRAs) in general and also the accomplishments achieved during the 6 month LDRD.

Risk calculations should focus on providing best estimate results and associated insights for evaluation and decision-making. Specifically, SPRAs are intended to provide best estimates of the various combinations of structural and equipment failures that can lead to a seismic induced core damage event. However, in some instances the current SPRA approach has large uncertainties and potentially masks other important events (for instance, it was not the seismic motions that caused the Fukushima core melt events but the tsunami ingress into the facility).

SPRAs are performed by convolving the seismic hazard (the frequency of certain magnitude events) with the seismic fragility (the conditional probability of failure of a structure, system, or component [SSC] given the occurrence of earthquake ground motion). In this calculation, there are three main pieces to seismic risk quantification: seismic hazard and nuclear power plants (NPPs) response to the hazard, the fragility or capacity of SSC, and systems analysis. Figure 1 provides a high level overview of the risk quantification process.

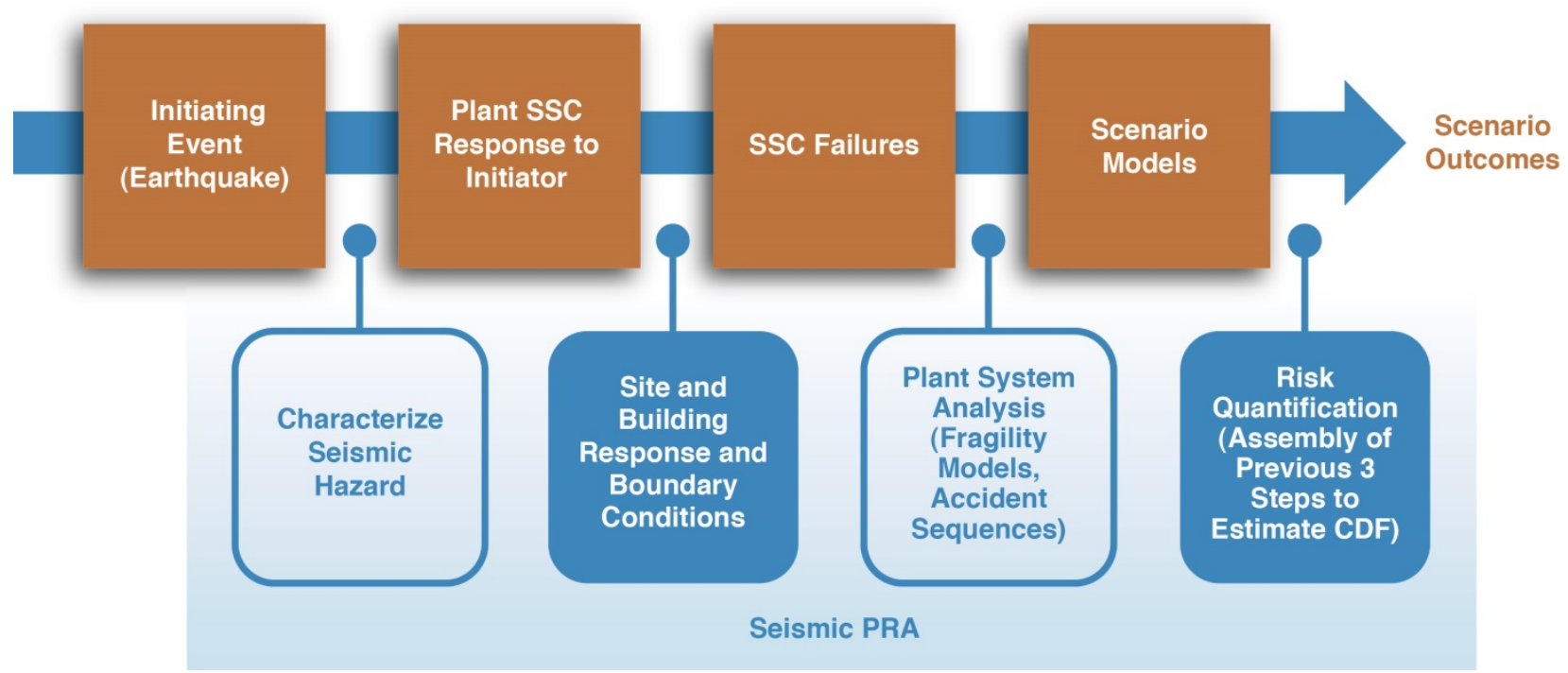

Figure 1. Seismic risk quantification process. 


\section{Goal: Provide a best estimate understanding of seismic risk at nuclear power plants by using high fidelity tools to model nuclear facility response to large seismic events.}

The focus of this research is on realistic modeling and simulation that emphasizes removing uncertainties (when possible) in the quantification of seismic risk at NPPs.

Risk analysts performing traditional SPRA calculations for NPPs make assumptions that create uncertainties. This research will provide methods, tools, and data that can be used to remove or minimize these uncertainties. Some assumptions that simplify the problem and create uncertainty in the calculation are:

- NPP response scales linearly with ground motion. This assumption affects the "Plant SSC Response to Initiator" box in Figure 1 where we see that as the earthquake intensity increases, the NPP response increases in a non-linear fashion.

- R\&D solution: Determine the degree of reasonableness of this assumption by evaluating and tracking the response of a generic NPP to increasing levels of ground motion.

- R\&D solution: Calculate the seismic core damage frequency using a traditional SPRA approach that uses linear seismic analysis combined with an advanced SPRA approach that uses Non-Linear Seismic Soil Structure Interaction.

- The Uniform Hazard Spectrum is used to define ground motions for determining NPP in-structure response. The Uniform Hazard Spectrum is conservative since it is an envelope of all ground motions. (This is the "Characterize Seismic Hazard" box in Figure 1.)

- R\&D Solution: Evaluate the change in seismic core damage frequency when using ground motions that are produced from a conditional mean spectrum, which is more representative of actual earthquakes (not an envelope of all earthquakes for a given site).

- Parameters that are weakly correlated to the damage of that SSC are used to define SSC fragility curves. For instance, peak ground acceleration is typically used to define failure of an SSC when this parameter is weakly correlated to damage of SSCs. (This is the "SSC Failures" box in Figure 1.)

- R\&D Solution: Study the impact on the system and component probability of failure when using the current practice with fragility curves that base probability of failure on peak ground acceleration versus fragility curves that have a strong correlation to failure of that system or component, such as differential displacement between components in the facility. 


\section{Summary}

This LDRD was converted to direct funding approximately 6 months into the FY at which time the LDRD was discontinued. Activities accomplished under the 6 month LDRD include:

- Implemented the Newmark-Beta Time Integration Method in MOOSE.

- Added structural dynamics capability into MOOSE with inclusion of a Newmark-Beta time integrator. This provides the capability to solve structural dynamics problems using MOOSE and solves the second order differential structural dynamics equation:

$$
M a_{n+1}+C v_{n+1}+K d_{n+1}=F_{n+1}
$$

- Identified a system for the NPP demonstration project.

The selected representative NPP structure is a pressurized water reactor building example. It consists of a pre-stressed concrete containment structure and reinforced concrete internal structure. The structure and its stick model representation are shown in Figure 2.

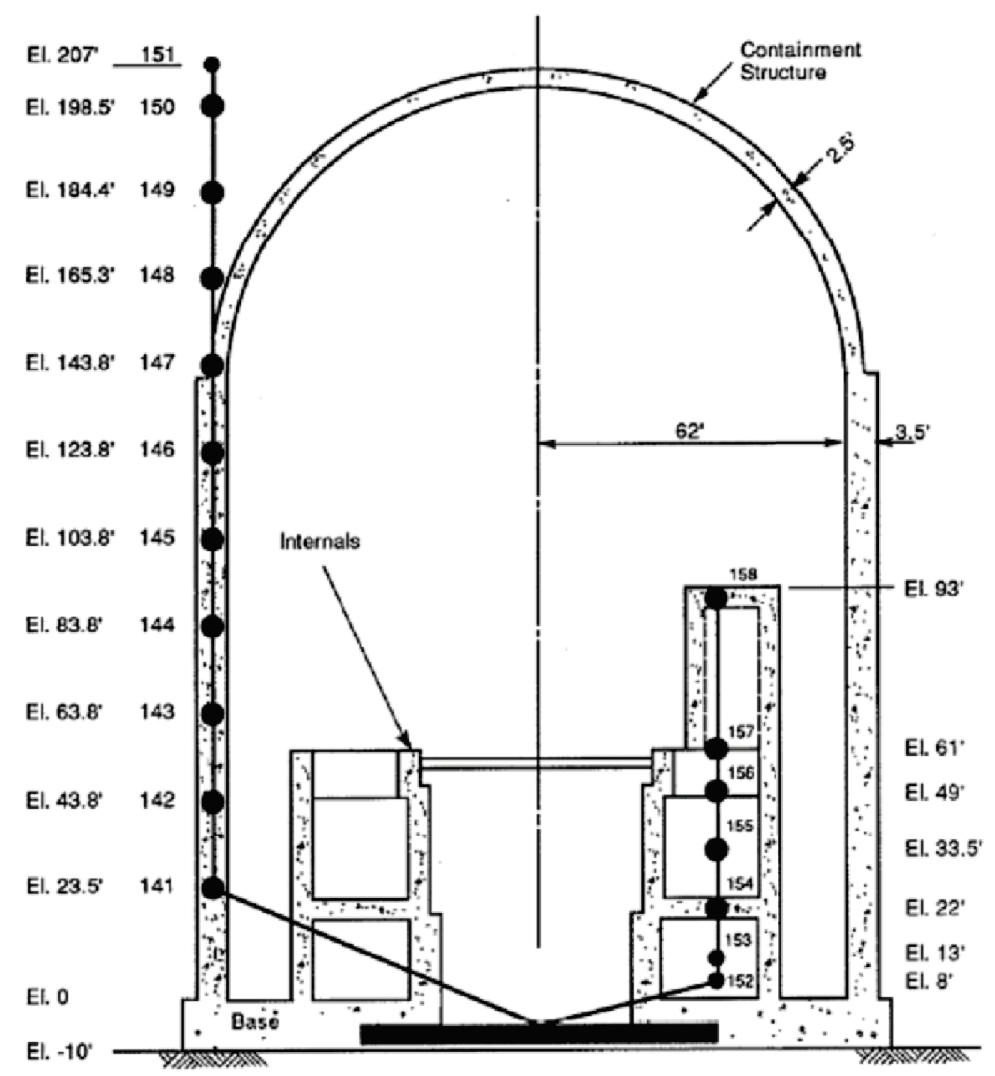

Figure 2. Stick model of the representative NPP structure.

- Performed a scoping test to demonstrate propagation of motion through a stochastic material property (this could represent the soil) using RAVEN. 


\section{Benefits to DOE}

SPRAs are intended to provide best estimates of the various combinations of structural and equipment failures that can lead to a seismic-induced core damage event and the integration of these results to quantify the risk. The advanced SPRA tools and methods outlined in this report propose to increase the fidelity of the SPRA methodology by using high fidelity modeling and simulation tools to provide more realistic seismic facility calculations for given earthquake events. This approach will provide more realistic seismic accelerations at locations in the nuclear facility where critical components are located.

NPP owners who are resolving Near Term Task Force Recommendation 2.1 may find that the traditional approach to SPRA produces core damage frequency numbers that are above the Nuclear Regulatory Commission allowable limit. The proposed advanced SPRA methodology and tools necessary to implement this methodology would provide the industry with more realistic SPRAs and may allow NPP owners to meet Near Term Task Force Recommendation 2.1. Additionally, these advanced tools and methods would provide for longer-term industry needs as they "confirm" their seismic hazards every 10 years.

\section{Publications}

Coleman, J., M. Talaat, P. Hashimoto, and C. Smith, "Advanced Seismic Probabilistic Risk Assessment Using Nonlinear Soil-Structure Interaction Analysis," PSA 2015, Sun Valley, ID, April 26-30, 2015.

\section{Presentations}

Coleman, J., "Advanced Seismic Probabilistic Risk Assessment for Nuclear Facilities," Presentation to DOE-Headquarters, August 2, 2014.

Coleman, J., "Advanced Seismic Probabilistic Risk Assessment for Nuclear Facilities," Presentation to DOE Assistant Secretary of NE Pete Lyons, May 5, 2014. 


\title{
14-104 - Development of a Multiphysics Algorithm for Analyzing the Integrity of Nuclear Reactor Containment Vessels Subjected to Extreme Thermal and Overpressure Loading Conditions
}

\author{
Richard C. Martineau, Ben Spencer, Ray Berry, David Andrs, Hongbin Zhang, and Curtis Smith
}

One of the physical needs to be considered for analyzing the integrity of nuclear reactor containment vessels subjected to extreme thermal and overpressure loading conditions is radiative heat transfer. Radiative heat transfer has wider application and has been studied extensively in the past, for example, by Michael F. Modest in "Radiative Heat Transfer." Radiative heat transfer through matter can be modeled by the radiative transfer equation (RTE) coupled with the material temperature or energy transfer equation through the black body emission and the temperature dependent material data. The energy transfer equation can be fairly complicated by itself if fluid dynamics is involved. We currently only consider the thermal conduction along with the radiation heating. It is noted that situations exist where radiation is the dominant mode of heat transfer so that the conduction and convection are both negligible. Efficiently solving the frequency-dependent transport equation is a long-standing problem. We have to approximate the equation to achieve simulation results with reasonable computing efforts, including the multigroup approximation. With multigroup approximation, the entire frequency range of interest can be split into multiple non-overlapping bands or groups. Then integration of the RTE over these groups can be performed, creating a set of equations without the frequency variable. The material properties, including the opacity, emissivity, and scattering cross sections in these equations, can be obtained with their continuous values weighted by the black-body emission spectrum or the approximated radiation spectrum. Boundary conditions for RTE can be applied with the boundary emissivities, reflectivities, and absorptivities.

\section{Summary}

Rattle $S_{N a k e}$ is a MOOSE-based application initially designed as a neutron transport solver. Various discretization schemes have been implemented for solving the multigroup neutron transport equation. The neutron transport equation and the RTE share some similarities but also differ from each other. They both have the streaming direction as the independent variable. RTE has a similar time derivative kernel, streaming kernel, collision kernel, and scattering kernel. (The name "kernel" is borrowed from the MOOSE framework, in which a kernel stands for a piece in the weak form for a particular term in the equation.) The new kernels introduced in RTE include the black-body emission kernel and the spatial varying refractive index kernel. We frequently solve the linear eigenvalue problem for neutron transport. On the other hand, RTE is typically coupled with the energy transfer equation in a strong nonlinear fashion. Nonlinear transient solvers are required for solving RTE. Outputs for modeling radiative heat transfer can be maximized by using RattleSNake/MOOSE with minimum effort. Rattle $S_{N}$ ake was extended for radiative heat transfer with all discretization schemes, solvers, and so on to be available to solve the multigroup RTE.

The need to solve large coupled systems of equations also required optimizations to some of the internal interfaces within MOOSE itself. In particular, optimal preconditioning of the RTE system requires building many small sub-matrices of the Jacobian matrix. These individual "blocks" of the Jacobian matrix can then be used either individually or collectively to precondition the system. The existing MOOSE interfaces only allow building one sub-matrix at a time, requiring a full "sweep" (evaluation of finite element shape functions, variables, and 
material properties on each element in the mesh) for each sub-matrix to be built. In a situation with $\mathrm{N}$ variables and $\mathrm{M}$ blocks to build, this would require $\mathrm{N} \times \mathrm{M}$ sweeps. The strong coupling between variables in RTE requires building many "off-diagonal" sub-matrices so that $\mathrm{M}$ converges to $\mathrm{N}$, giving an $\mathrm{O}\left(\mathrm{N}^{2}\right)$ algorithm to build the matrices for preconditioning. To alleviate this, a new interface was added to MOOSE that allows building multiple sub-matrices in one pass through the mesh, effectively reducing the computational complexity to $\mathrm{O}(\mathrm{N})$.

The implementation of RTE in RattleS $S_{N a k e}$ has been tested with few benchmark problems, including the non-equilibrium Marshak wave problem, a one-group coupled problem obtained from G. C. Pomraning with our self-adjoint angular flux with discrete ordinates method and continuous finite element method transport scheme. Here we will only show part of the results for the non-equilibrium Marshak wave problem. Others, including results for the other benchmark problem, can be found in our detailed report "Radiative Heat Transfer with RattleS $S_{\text {ake." }}$ The problem was proposed by Pomraning, and its solution is later extended by Su and Olson. Details about this problem can be found in "Benchmark Results for the Non-Equilibrium Marshak Diffusion Problem" by Su Bingjing and Gordon L. Olson and "Radiative Heat Transfer in Inhomogenous, Nongray, and Anisotropically Scattering Media" by Zhixiong Guo and Shigenao Maruyama. In our simulations, we made the medium finite size and applied the vacuum boundary condition on the new boundary. We properly set the parameters so that the

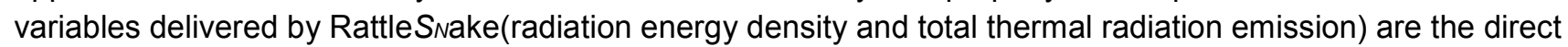
variables in the problems. The initial time step size was set to $10^{-18}$ seconds. At the first time step, the mesh was adapted so that no oscillations in the solution would be observed, which resulted in 233 elements mostly refined at the boundary with the non-zero incoming flux. Both spatial adaptivity and time adaptivity were turned on with the Transient executioner in MOOSE. The time stepper in use was SolutionTimeAdaptiveDT and the error indicator was GradientJumpIndicator with the refinement fraction being 0.01 and coarsening fraction being 0.5 . The simulation is completed in 827 steps at time equal to 0.001 seconds. Because the calculation was done with the finite domain, we cannot reproduce the reference solution exactly. However, the boundary effect to the solutions on the left boundary is small, so we can still make meaningful comparison to the reference solution there. The results are showed in Table 1. While the solutions are indeed close, a consistent lower solution in our simulation can be observed due to the finite domain size.

Table 1. Solutions of the Marshak wave problem at the left boundary with $e=0.1$.

\begin{tabular}{|c|c|c|c|c|}
\hline \multirow{2}{*}{$t$} & \multicolumn{2}{|c|}{$u(x=0, t)$} & \multicolumn{2}{c|}{$v(x=0, t)$} \\
\cline { 2 - 5 } & $\begin{array}{c}Z_{\max }=20, \\
\text { RattleSNake }\end{array}$ & $\begin{array}{c}z_{\max }=¥, \\
\text { Reference }\end{array}$ & $\begin{array}{c}z_{\max }=20, \\
\text { RattleSNake }\end{array}$ & $\begin{array}{c}Z_{\max }=¥, \\
\text { Reference }\end{array}$ \\
\hline 0.001 & 0.08988 & 0.09039 & 0.00006 & 0.00006 \\
\hline 0.003 & 0.14686 & 0.14768 & 0.00031 & 0.00030 \\
\hline 0.01 & 0.23931 & 0.23997 & 0.00171 & 0.00170 \\
\hline 0.03 & 0.34235 & 0.34328 & 0.00766 & 0.00762 \\
\hline 0.1 & 0.43768 & 0.43876 & 0.03452 & 0.03446 \\
\hline 0.3 & 0.48526 & 0.48556 & 0.11298 & 0.11322 \\
\hline 1 & 0.55141 & 0.55182 & 0.31848 & 0.32030 \\
\hline 3 & 0.66212 & 0.66334 & 0.58561 & 0.58906 \\
\hline 10 & 0.79215 & 0.79420 & 0.78045 & 0.78318 \\
\hline 30 & 0.87612 & 0.87731 & 0.87395 & 0.87523 \\
\hline 100 & 0.93169 & 0.93202 & 0.93135 & 0.93167 \\
\hline
\end{tabular}


The focus for the remainder of the project will be on the following tasks:

1. Add more tests and benchmark problems to verify the implementation

2. Add material data or the cross sections with existing high-resolution spectroscopic databases

3. Create a more realistic benchmark for analyzing the integrity of the nuclear reactor containment vessels subjected to extreme thermal and over pressure loading conditions.

\section{Benefits to DOE}

This work is directed to create a new algorithm for thermal-mechanical fluid-structure interaction to address structural integrity in the presence of thermal radiation, natural and forced convection, and strong hydrodynamic forces. While this effort is directed with nuclear safety for nuclear power plants in mind, applications for nuclear security are numerous. 


\section{NATIONAL AND HOMELANSEURITr}




\section{2-001 - Cyber Security for 4th Generation Cellular Communications}

\section{Kurt Derr and Sam Ramirez}

In the next few years, Long Term Evolution (LTE) technology will become pervasive in industry, government, and intelligently controlled critical infrastructure, as it offers a high-speed, high-bandwidth telecommunication option that enables new human-to-machine and machine-to-machine applications. As development of LTE compatible mobile devices moves forward, more software applications will need enhanced security to reduce the impact of a cybersecurity event targeting asset owners and end users. INL is performing this research to secure wireless communications systems from cyber-attacks against devices using LTE technology. The objectives of this LDRD are to (1) investigate, analyze, and test new fourth generation (4G) LTE devices to determine the degree to which cybersecurity vulnerabilities of $3 G$ systems are resolved by new $4 G$ protocols, as well as the degree to which vulnerabilities exist in the design and implementation of $4 G$ protocols; and (2) mitigate any vulnerabilities discovered, to the extent possible, in LTE protocol implementations.

\section{Summary}

A lab-scale LTE R\&D system (Figure 1) was designed and constructed to conduct research experiments on cellular security and cellular communications issues. This R\&D system is capable of interfacing to WiMAX, WiFi, 2G, 3G, and LTE systems. The research performed this year utilized the LTE and WiFi network interfaces to demonstrate applications for voice, video, text, and file transfer; Android to Android smart phone communications using video and voice; and Android smart phones as user equipment (UE) to demonstrate handovers from WiFi to LTE and LTE to WiFi.

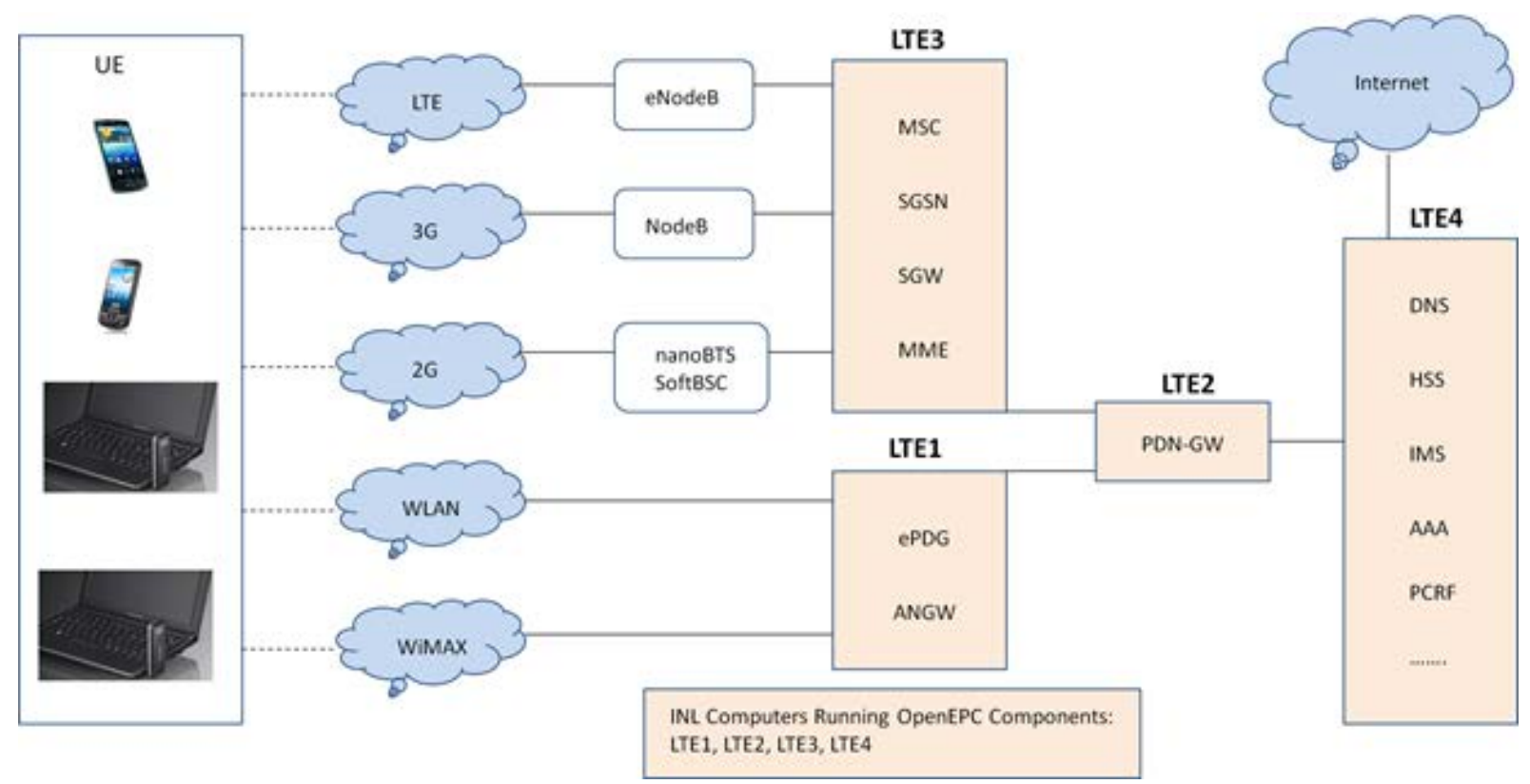

Figure 1. The LTE R\&D system was designed and constructed to conduct experimentation with LTE1, LTE2, LTE3, and LTE4 computers and legacy wireless communication technologies. 
The experimental methodology included identifying UE applications/use cases that exercise the communications functionality of the LTE system, running UE applications while monitoring communications in the LTE system, and utilizing protocol fuzzing or other techniques to discover potential vulnerabilities while running UE applications. Other experiments involved code modifications to evaluate Denial of Service threats and signal interference to determine the potential for both Denial of Service and performance degradation. Work was initiated that explored the feasibility of alternative approaches to conduct Denial of Service attacks on LTE systems.

The consequences associated with potential vulnerabilities were characterized using the Microsoft DREAD method (ㅁamage potential, Reproducibility, Exxploitability, Affected users, and Discoverability). Experimental results of the effectiveness of different cyber-attack methods were evaluated and ranked related to the effort to exploit the system and discover that an attack was underway. The project research results indicate that LTE systems can benefit from further cybersecurity research experimentation to assure operations for government and public safety communications. Concepts for enhancing the security of LTE systems are proposed for evaluation during follow-on research.

\section{Benefits to DOE}

This research initiative contributes directly to DOE's goal of securing critical infrastructure from cyber attacks. The LTE experimentation results and R\&D system will provide a foundation for wireless communications-cybersecurity research to enhance the security of commercial off-the-shelf wireless communication systems. 


\title{
13-060 - Metal Fluoride Preparation for AMS Analysis
}

\author{
James E. Delmore, Gary S. Groenewold, and Chris A. Zarzana
}

INL researchers are developing new chemical approaches that will support measurements of isotope ratios, which are used by the U.S. and international organizations to ensure that nuclear energy facilities are operating in compliance with nonproliferation treaty commitments. The project strategy is to develop new means for converting elemental isotopes in environmental samples into negatively charged, fluorine $(F)$-containing molecules called "fluoroanions" that are compatible with existing and emerging isotope ratio mass spectrometry instrumentation. If successful, the project will provide the scientific chemical basis for improved ultratrace isotope ratio measurement having better accuracy, lower detection limits, and reduced costs.

\section{Summary}

Experimental results from this research have demonstrated that unique fluoroanions of uranium (U), zirconium ( $\mathrm{Zr}$ ), iron ( $\mathrm{Fe}$ ), and silicon ( $\mathrm{Si}$ ) can be produced in high abundance with very low chemical background. These fluoroanions were produced by reacting oxides and salts of the metals with an ionic liquid, 1-ethyl-3-methylimidazolium fluorohydrogenate (EMF). After dilution with acetonitrile to make fluoroanion solutions, these compounds were analyzed using either electrospray ionization- or desorption chemical ionization-mass spectrometry. The EMF sample was provided by the research team of Tetsuya Tsuda (Osaka University) and Rika Hagiwara (Kyoto University).

Preliminary experiments showed that both $\mathrm{Zr}$ and $\mathrm{Fe}$ anions could be produced merely by mixing salts with the EMF ionic liquid and then diluting the resulting solution for electrospray ionization mass spectrometry analyses. The zirconyl cation $\mathrm{ZrO}^{2+}$ reacted to form $\mathrm{ZrF}_{5}^{-}$in an efficient manner, demonstrating that the fluorohydrogenate anion had the potential of being a good candidate for removing strongly bound oxo-functional groups, which can complicate isotope ratio measurements of actinide elements. In the case of $\mathrm{Fe}$, analyses of both $\mathrm{Fe}^{3+}$ and $\mathrm{Fe}^{2+}$ salts resulted in production of $\mathrm{FeF}_{4}{ }^{-}$, which further confirmed the potential application for actinide measurements.

Further experimentation to understand the future applications for $U$ measurements determined that $U$ oxides can be easily dissolved in the ionic liquid without using an oxidizing mineral acid. The resulting solution generated an exceptionally clean mass spectrum consisting principally of $\mathrm{UF}_{6}{ }^{-}$at $\mathrm{m} / \mathrm{z} 352$, with smaller ions at $\mathrm{m} / \mathrm{z} 333$ and 103 that correspond to $\mathrm{UF}_{5}{ }^{-}$and $\mathrm{AlF}_{4}{ }^{-}$(Figure 1). The analyses, conducted using an electrospray-ionization time-of-flight mass spectrometer, demonstrated sufficient dynamic range to enable measurement of the $\mathrm{U}-235$ isotopic ion and the need for more experimentation to measure the low intensity ions at neighboring $\mathrm{m} / \mathrm{z}$ values. 

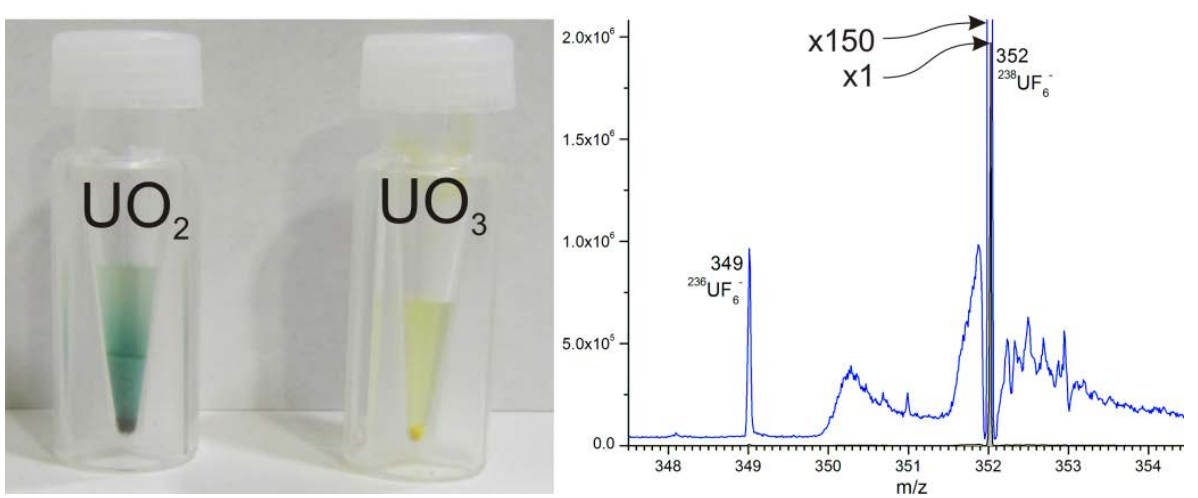

Figure 1. Left, small volumes of $\mathrm{UO}_{2}$ and $\mathrm{UO}_{3}$ dissolved in [EMIm][FHF]. The intense blue color is indicative of $U_{6}$. Right, negative ion mass spectrum; the black trace is plotted on scale, the blue trace shows same data, and the $y$ axis is expanded by 150x.

\section{Benefits to DOE}

Transitioning the fluoroanion formation chemistry into an accurate analytical isotope-ratio measurement method will enable the U.S. to make more informed nonproliferation policy decisions regarding the nonproliferation status of international nuclear energy programs at a substantially reduced cost compared with current methods. Improved measurement of isotope ratios at the ultratrace level will advance the DOE nonproliferation mission and elevate the global recognition of DOE's leadership position in nuclear and radiological science and measurement technologies.

\section{Publications}

Zarzana, C., G. Groenewold, M. Benson, J. Delmore, T. Tsuda, and R. Hagiwara, "Iron Fluoroanions and Their Clusters by Electrospray lonization of a Fluorinating lonic Liquid," J. Am. Soc. Mass Spectr., 2014, submitted.

Groenewold, G., J. Delmore, M. Benson, T. Tsuda, and R. Hagiwara, "Generation of Gas Phase Zirconium Fluoroanions by Electrospray of an Ionic Liquid," Rapid Commun. Mass Sp., Vol. 26, 1233-1242, 2014.

Groenewold, G., J. Delmore, M. Benson, T. Tsuda, and R. Hagiwara, "Fluorohydrogenate Cluster lons in the Gas Phase: Electrospray lonization Mass Spectrometry of the [1-ethyl-3-methylimidazolium $\left.{ }^{+}\right]\left[\mathrm{F}(\mathrm{HF})_{2.3}{ }^{-}\right]$lonic Liquid," J. Phys. Chem. A, Vol. 117, 14191-14199, 2013. 


\title{
13-092 - Fission Product Standard Production
}

\author{
Jana Pfeiffer, Kevin Carney, Leigh Martin, and Christopher McGrath ${ }^{1}$
}

The global national security community has a need for fission product standards to support the detection capabilities of nonproliferation, counterproliferation, and nuclear forensics programs. Development of fast spectrum fission product standards presents interesting research challenges in methods for isotope production, separation, and verification measurements. This project is pursuing a novel methodology of utilizing the spontaneous fission decay of californium (Cf)-252 as an alternative source of fission products to irradiation in a reactor. Successful collection of $\mathrm{Cf}-252$ particulate fission products, followed by chromatographic separation to isolate desired isotopes, could provide a viable solution to production of fast-fission standards in an efficient, cost-effective, and safe manner.

\section{Summary}

The design for a system capable of collecting both gaseous and particulate fission products resulting from the spontaneous fission of Cf-252 was adapted from an apparatus used by R. A. Anderl, V. J. Novick, and R. C. Greenwood for introduction of collected fission products into a mass separator that was located at INL ATR. This system was installed in the Analytical Laboratory at MFC and has been used to demonstrate the collection and purification of xenon (Xe)-135 by C. A. McGrath, T. P. Houghton, J. K. Pfeiffer, and R. K. Hague, in "Xe-135 Production from Cf-252." Research in the collection and analysis efficiencies of other Cf-252 fission products will be conducted after successful development of a method for separation of rare earth isotopes.

To develop this separation methodology, FY 2013 research focused on drip column separations of yttrium (Y), neodymium ( $\mathrm{Nd}$ ), samarium $(\mathrm{Sm})$, europium $(\mathrm{Eu})$, and terbium $(\mathrm{Tb})$ (Figure 1). Limitations in the effectiveness of drip-column separations for the tracer level amount of fission products produced by the Cf-252 source being used for this development work led to FY 2014 focus on characterization of a high-purity liquid chromatography separation scheme, utilized by R.G. Fernandez and J.I. Garcia Alonso in the Journal of Chromatography A, to enable the successful demonstration of the collection and separation of lanthanide fission products, as well as the determination of the decontamination factors for $\mathrm{Cf}-252$ and curium $(\mathrm{Cm})-248$. Three replicate surrogate solutions of lanthanide elements $\mathrm{Y}$, lanthanum (La), cerium (Ce), praseodymium ( $\mathrm{Pr}$ ), Nd, Sm, Eu, gadolinium (Gd), Tb, and dysprosium (Dy) were separated using this scheme, and inductively coupled plasma mass spectrometry analysis indicated that this separation can be performed with good repeatability (Table 1).

\footnotetext{
${ }^{1}$ Idaho State University
} 


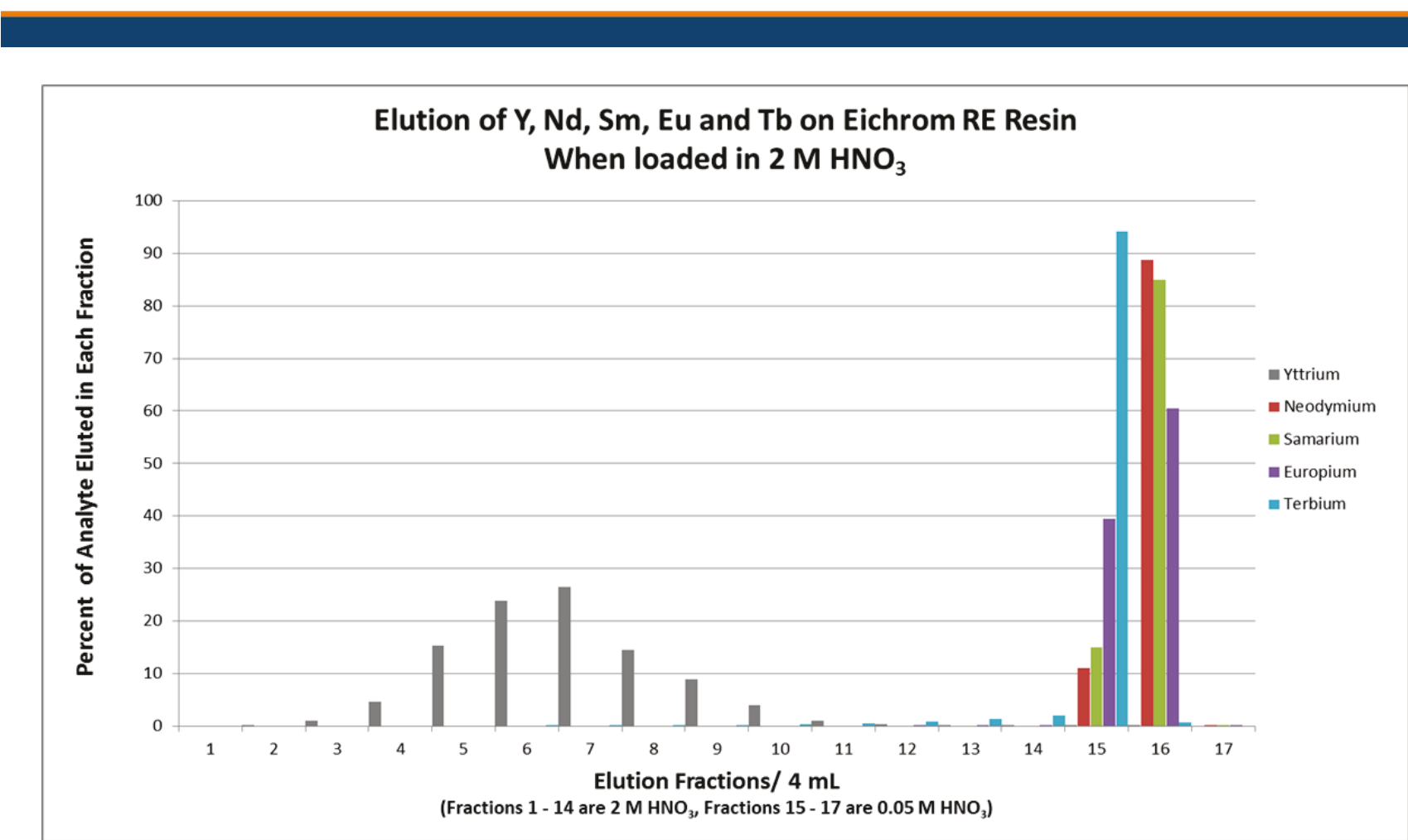

Figure 1. A solution containing stable $\mathrm{Y}, \mathrm{Nd}, \mathrm{Sm}$, $\mathrm{Eu}$, and Tb in $2 \mathrm{M} \mathrm{HNO}_{3}$ was loaded on a drip column containing Eichrom's RE resin (1M CMPO in TBP on an inert support). Yttrium was retained and eluted in $2 \mathrm{M} \mathrm{HNO}_{3}$, then the remaining lanthanides were eluted as a group in $0.05 \mathrm{M} \mathrm{HNO}_{3}$.

Table 1. Elemental purity and yield of selected elution fractions.

\begin{tabular}{|c|c|c|c|}
\hline Element & Fraction & Purity & Yield \\
\hline & & $(\%)$ & $(\mathbf{\%})$ \\
\hline Yttrium & 5 & $100 \pm 0$ & $99 \pm 0.28$ \\
\hline Lanthanum & 7 & $54 \pm 1.4$ & $92 \pm 2.9$ \\
\hline Cerium & 11 & $98 \pm 0.85$ & $90 \pm 1.3$ \\
\hline Praseodymium & 14 & $90 \pm 0.51$ & $74 \pm 1.2$ \\
\hline Neodymium & 17 & $99 \pm 0.10$ & $50 \pm 0.27$ \\
\hline Samarium & 24 & $97 \pm 0.20$ & $66 \pm 7.6$ \\
\hline Europium & 22 & $100 \pm 0.24$ & $58 \pm 8.3$ \\
\hline Gadolinium & 18 & $93 \pm 1.5$ & $94 \pm 0.49$ \\
\hline Terbium & 12 & $96 \pm 0.11$ & $22 \pm 10$ \\
\hline Dysprosium & 8 & $77 \pm 6.0$ & \\
\hline
\end{tabular}


By employing fraction picking (collecting only high purity eluent fractions in the separation and neglecting the overlapping fractions at the expense of yield), most of the elements tested can be isolated with $93 \%$ or greater purity with yields of at least $90 \%$ (Figure 2). Changes in elution order and retention time effected by increases in the $\mathrm{pH}$ of the eluent could be used to optimize the purity and yield for elements whose elutions directly overlapped when the separation was performed at $\mathrm{pH} 6.5$ (Figure 3). Further experiments where concentration of the analytes was reduced to more closely represent the expected yield of fission products from the Cf-252 source being used indicated that stable element carriers might be necessary to predictably purify the radioactive isotopes collected.

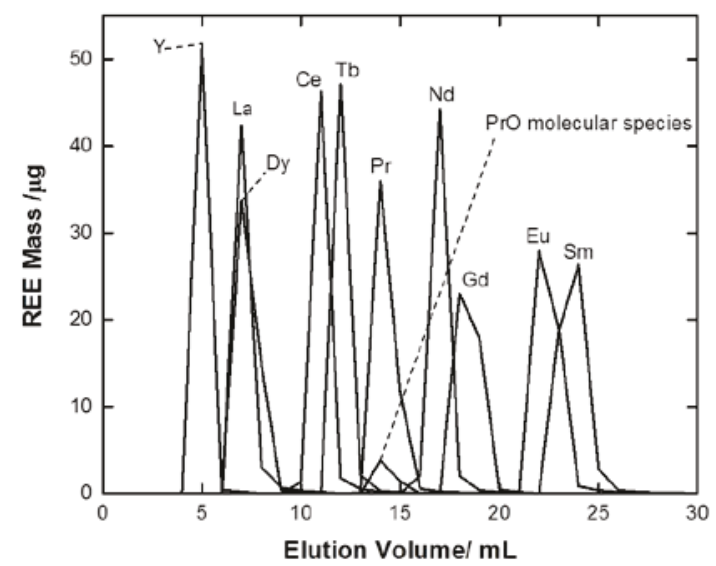

Figure 2. Surrogate solution of $50 \mu \mathrm{g}$ of each lanthanide separated using Dionex AS9-HC column, $5 \mathrm{mMol}$ EDTA eluent at $\mathrm{pH} 6.5$.

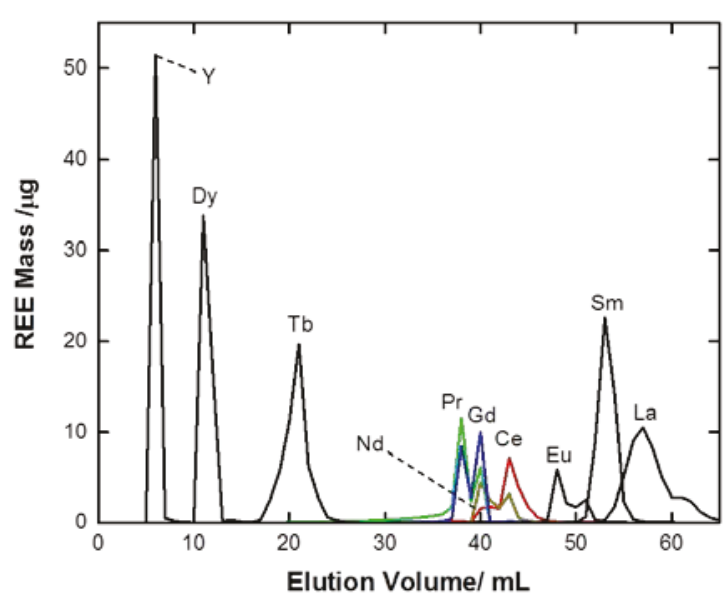

Figure 3. Surrogate solution of $50 \mu \mathrm{g}$ of each lanthanide separated using Dionex AS9-HC column, $5 \mathrm{mMol}$ EDTA eluent at $\mathrm{pH} 9$.

\section{Benefits to DOE}

Successful collection and separation of fission products from the spontaneous fission of Cf-252 supports DOE objectives to expand scientific knowledge and laboratory capabilities to conduct research and develop technology solutions for arms control and treaty verification. Capabilities developed may expand the suite of pure isotopes that can be provided for the Comprehensive Test Ban Treaty and National Nuclear Technical Forensics communities. This work will also support the Federal Bureau of Investigation in the collection of material samples and other forensics data in support of domestic post-detonation ground debris collection capabilities. 


\title{
13-093 - Spectrum Allocation and Communications in Dynamic Spectrum Access Channels
}

\author{
Hussein Moradi, David Couch, Brandon Lo, Daryl Wasden, and Behrouz Farhang-Boroujeny ${ }^{1}$
}

The exponential growth of wireless devices and mobile data usage in our nation demands additional spectrum or flexible use spectrum for high-speed wireless broadband access. It is predicted in the Ericsson Mobility Report on the Pulse of the Networked Society that there will be a seven-fold mobile data traffic increase in North America between 2013 and 2019. To meet such a strong demand, the congressional directive in the Spectrum Act of 2012, namely the Middle Class Tax Relief and Job Creation Act of 2012, allows more spectrum bands available for flexible uses, which enables spectrum sharing among federal and commercial users. Moreover, the President's Memorandum of 2013 states that spectrum sharing should be used to enhance efficiency among all users subject to interference protection for federal users, especially national security, law enforcement, and public safety. Nevertheless, the spectrum sharing arrangements with federal incumbents pose a new challenge of achieving the efficiency of spectrum utilization while protecting the operations of federal users from harmful interference.

The objective of this LDRD is to address this challenging spectrum sharing problem by a novel dynamic spectrum access (DSA) solution to assure seamless and secure communications for federal users. According to Qing Zhao and B.M. Sadler in "A Survey of Dynamic Spectrum Access," DSA has long been recognized as a promising solution for interference mitigation and the enhancement of spectrum utilization. However, many open challenges, such as efficient wideband spectrum sensing, effective spectrum allocation, and secure opportunistic access, remain in the realization of DSA. Therefore, this LDRD effort is focused on developing a new filter bank (FB) spectrum sensing technique for spectrum hole identification in a wideband spectrum, cognitive channel prediction algorithm for spectrum allocation, and FB multicarrier spread spectrum (FBMCSS) technology for secure and robust communications that demonstrate the feasibility of DSA as the solution to the new spectrum sharing challenges in a flexible use spectrum.

\section{Summary}

The proposed DSA solution provides secure communications, interference mitigation, and spectral efficiency to federal users in a flexible use spectrum. The DSA communications based on FBMCSS technology appear as noise to other federal or commercial users sharing the spectrum, which does not cause harmful interference to coexisting wireless systems. Moreover, the communications are robust to interference or malicious jamming owing to frequency spreading over wideband spectrum. As a result, this DSA solution achieves an unparalleled level of secure communications and coexistence. To identify the spectrum holes for opportunistic access, the FBMCSS receiver is also used for FB spectrum sensing, which does not incur additional implementation cost and complexity. More importantly, the FB structure is capable of sensing the wideband spectrum simultaneously in the order of milliseconds, thus considerably reducing the time required to sense and improve the efficiency of wideband spectrum sensing. Hence, compared to existing wideband spectrum sensing techniques developed by Hongjian Sun, A. Nallanathan, Cheng-Xiang Wang, and Yunfei Chen in "Wideband Spectrum Sensing for Cognitive Radio Networks: A Survey," the proposed DSA solution senses wideband spectrum more efficiently without incurring extra hardware cost and complexity.

\footnotetext{
${ }^{1}$ University of Utah
} 


\section{NATIONAL \& HOMELAND SECURITY}

With the information of wideband spectrum availability from FB spectrum sensing, the proposed DSA solution also provides cognitive channel prediction for effective spectrum allocation to minimize the possibility of interference and maximize the system capacity. The predictive algorithm at the heart of the proposed DSA solution is capable of predicting the futuristic traffic patterns and channel availability based on the previous observations and the current channel states. Thus, the spectrum that will be available with the highest probability for a long period in the future can be allocated effectively and safely for transmission to mitigate the possibility of unexpected harmful interference. Compared to many complex prediction methods, the proposed recursive algorithm is effective, yet easy to implement in hardware. Furthermore, the system parameters for prediction can adapt to the traffic pattern and transmission activity changes due to the dynamic nature of spectrum usage. This capability enhances the accuracy of channel prediction, thus greatly avoiding the potential collisions of transmission and improving spectrum utilization to the maximum degree. Therefore, the proposed DSA solution achieves high degree of interference mitigation and spectral efficiency enhancement and is the cost-effective and secure solution for federal users to share spectrum with all types of users in the flexible use spectrum.

Dr. Brandon Lo was an intern from Georgia Tech in 2010, he is now a full-time employee of INL and a major contributor for developing predictive algorithm in this research.

Dr. Daryl L. Wasden was initially a contributor to this LDRD as a Ph.D. Student from University of Utah; he is now a full-time employee of INL continuing the research in the DSA area.

\section{Benefits to DOE}

This research contributes to DOE's mission by enhancing the security of energy infrastructure and Public Safety communications by developing solutions to implement the U.S. Office of Science and Technology Policy "Presidential Memorandum-Unleashing Wireless Broadband Revolution." Additionally, this project will benefit federal agencies with operations in the 1755-1850 MHz bands, including the Departments of Energy, Defense, Homeland Security, and Justice as well as the Federal Aviation Administration and National Aeronautics and Space Administration.

\section{Publications}

Wasden, D., H. Moradi, and B. Farhang-Boroujeny, "Comparison of Direct Sequence Spread Spectrum Rake Receiver with a Maximum Ratio Combining Multicarrier Spread Spectrum Receiver," Proceedings of IEEE ICC 2014, June 2014.

Deaton, J., L. Brighton, R. Subramanian, H. Moradi, and J. Loera, "Accelerating Spectrum Sharing Technologies," IEEE Communications Magazine, Vol. 51, 118-122, September 2013.

Wasden, D., H. Moradi, and B. Farhang-Boroujeny, "Design and Implementation of an Underlay Control Channel for Cognitive Radios," IEEE Journal on Selected Areas in Communications, Vol. 30, 1875-1889, November 2012.

Wasden, D., J. Loera, H. Moradi, and B. Farhang-Boroujeny, "Design and Implementation of a Multicarrier Spread Spectrum Communication System," Proceedings of Military Communications Conference (MILCOM) 2012, 1-7, Orlando, FL, October 29-November 1, 2012. 
Wasden, D., J. Loera, A. Majid, H. Moradi, and B. Farhang-Boroujeny, "Design and Implementation of an Underlay Control Channel for Cognitive Radios," Proceedings of IEEE International Symposium on Dynamic Spectrum Access Networks (DYSPAN) 2012, 280-281, Bellevue, WA, October 16-19, 2012.

\section{Presentations}

Wasden, D., H. Moradi, and B. Farhang-Boroujeny, "Comparison of Direct Sequence Spread Spectrum Rake Receiver with a Maximum Ratio Combining Multicarrier Spread Spectrum Receiver," IEEE ICC SPC-09: OFDM and Multicarrier Systems, Sydney, Australia, June 13, 2014.

Moradi, H., "DEA Spectrum Communications Research: Video Surveillance," Drug Enforcement Administration (DEA) Presentation, July 29, 2013.

Moradi, H., "Anti-Jamming LPI-LPD-LPE Wireless Communications System," USSOCOM Presentation, June 24, 2013.

Moradi, H., "National Security/Emergency Preparedness: Technical Execution Discussion," Joint Program Office (JPO) Presentation, June 26, 2013.

Wasden, D., J. Loera, H. Moradi, and B. Farhang-Boroujeny, "Design and Implementation of a Multicarrier Spread Spectrum Communication System," Military Communications Conference (MILCOM), October 29-November 1, 2012.

Wasden, D., J. Loera, A. Majid, H. Moradi, and B. Farhang-Boroujeny, "Design and Implementation of an Underlay Control Channel for Cognitive Radios," IEEE International Symposium on Dynamic Spectrum Access Networks (DYSPAN), Bellevue, WA, October 16-19, 2012. 


\title{
13-118 - Geomagnetic Disturbance Field Coupling Measurements on INL Power Grid
}

\author{
Shawn West, Mack Grady, ${ }^{1}$ Scott McBride, Tom Baldwin, Carol Reid, Dave Fromme ${ }^{2}$
}

INL has tested the effects of geomagnetic disturbances (GMDs) caused by solar storms on critical power system components using the laboratory's full-scale power grid. This project measures and correlates geomagnetic storms with the resultant phenomena of Ground Induced Currents (GIC) onto INL's 138-kV transmission grid. Because electric utilities are unable to perform these tests on their operating systems, the INL power grid provides a unique opportunity for research and validation. Until INL's LDRD, utility companies have relied upon models and theories to help mitigate the possible effects of solar storms.

The project is obtaining GIC measurements from three-phase, alternating-current, high-voltage, and medium-voltage INL transformers, transmission lines, and power infrastructures simultaneously with collocated geomagnetic effect sensors (mass flow sensors). Current-flow sensors were placed on available transformer phases and ground circuits on the INL transmission line in time to observe the peak of the solar storm cycle that occurred from December 2013 through December 2014. Through the array measurements of electric and magnetic fields, detailed waveforms of the phase voltages, line, and neutral currents will be obtained. Characteristics of the waveforms will be used to develop methods for use by the electric utility industry to detect and protect against grid transformer damage caused by GIC resulting from GMD.

\section{Summary}

To support the establishment of INL as a leader in understanding and predicting the coupling effects of GIC into a utility-scale grid, this project's researchers developed and installed a GMD/GIC measurement recording system on the INL power grid (see Figures 1-3 below). The system measures and correlates GMD effects imposed by solar storms on the INL grid system, allowing INL engineers to understand the phenomena as it affects INL to determine what (if any) damaging effects are possible and to develop and test mitigations as necessary. The ability to understand, predict, and mitigate GMD/GIC problems on INL's grid will enable assisting the larger-scale utilities in understanding, predicting, and mitigating effects in the national grid system.

\footnotetext{
${ }^{1}$ Baylor University

${ }^{2}$ Scientific Applications \& Research Associates
} 


\section{NATIONAL \& HOMELAND SECURITY}

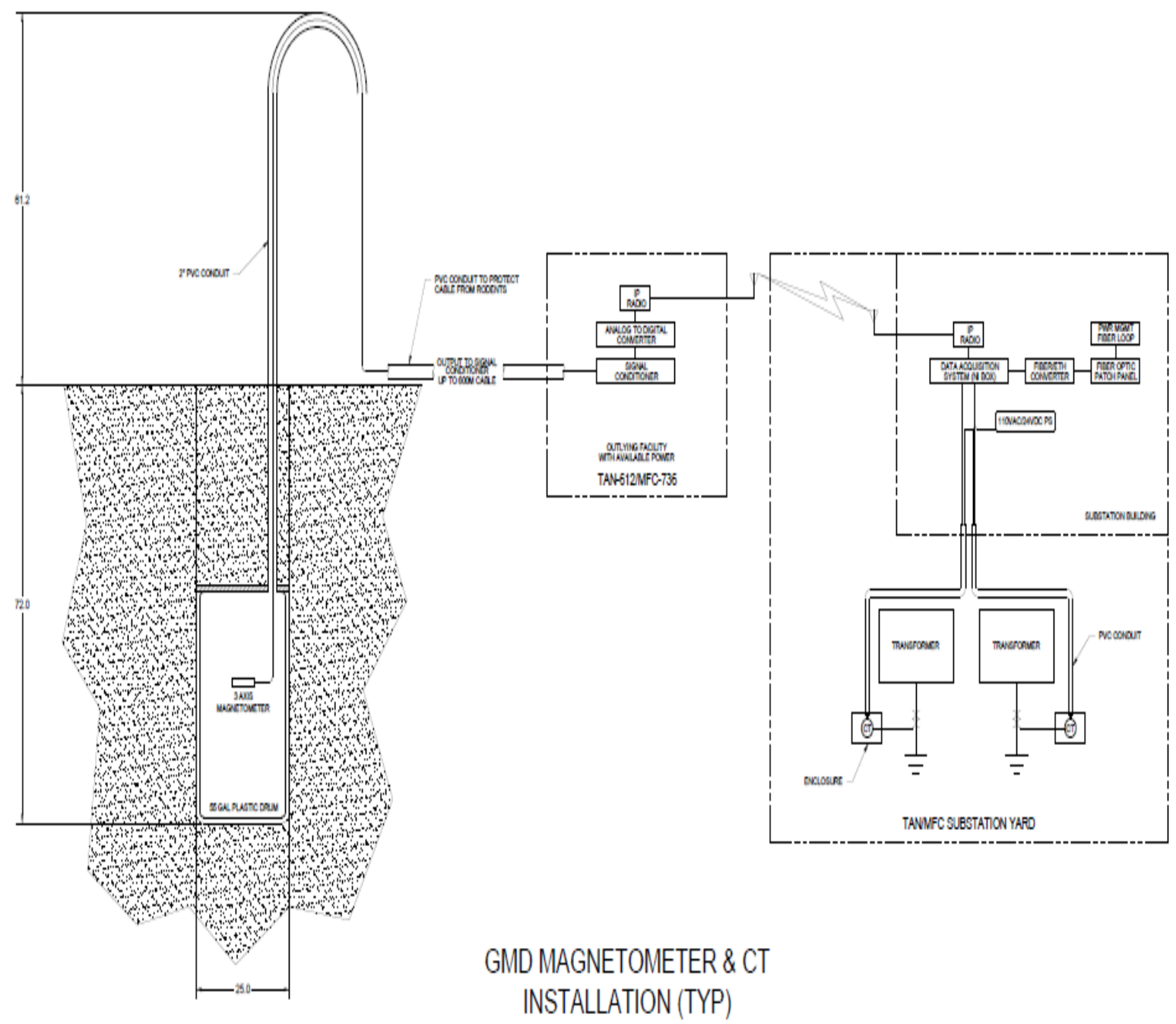

Figure 1. Block diagram of INL typcial GMD system layout. 


\section{NATIONAL \& HOMELAND SECURITY}

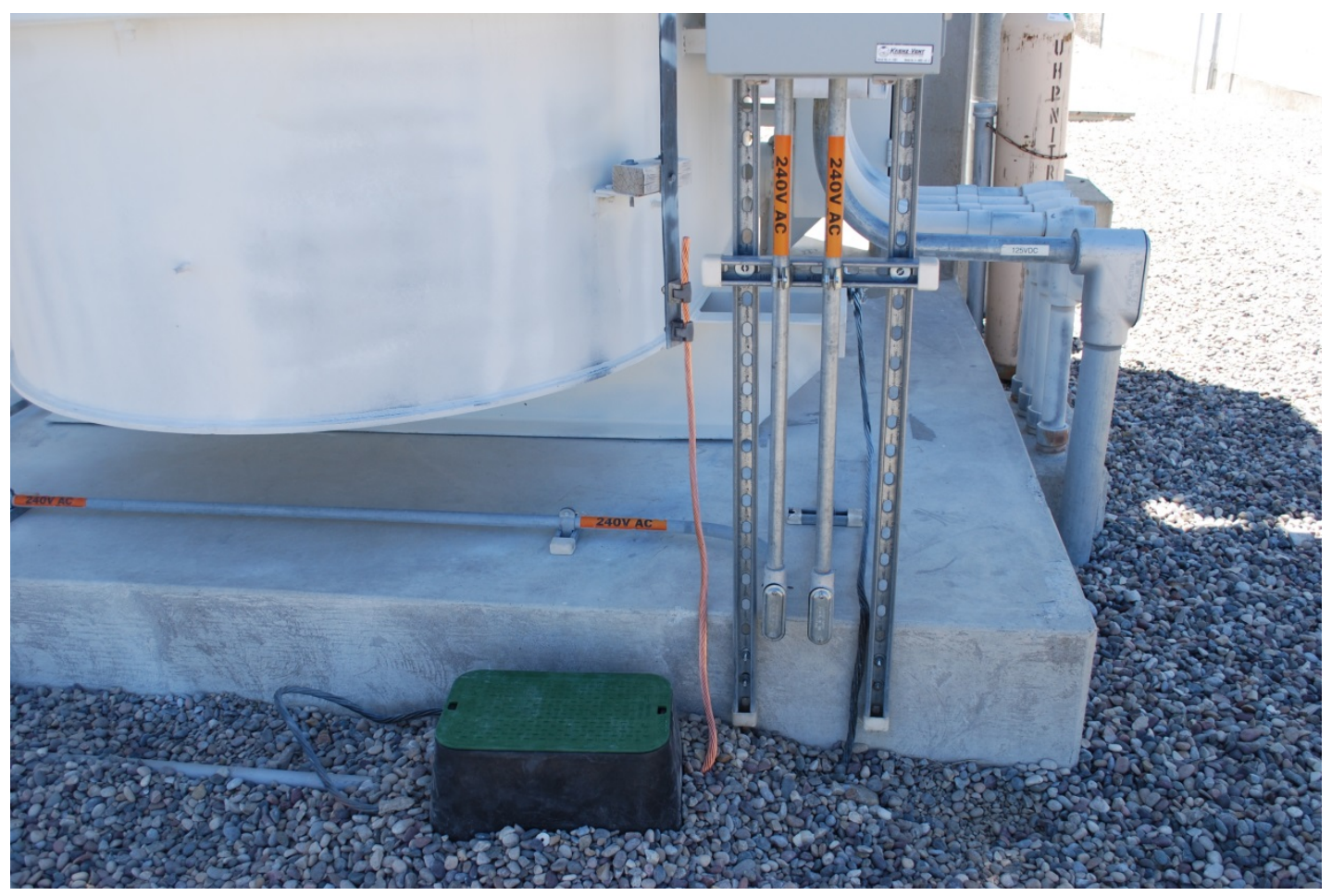

Figure 2. Typical INL transformer-GMD ground current measurement.

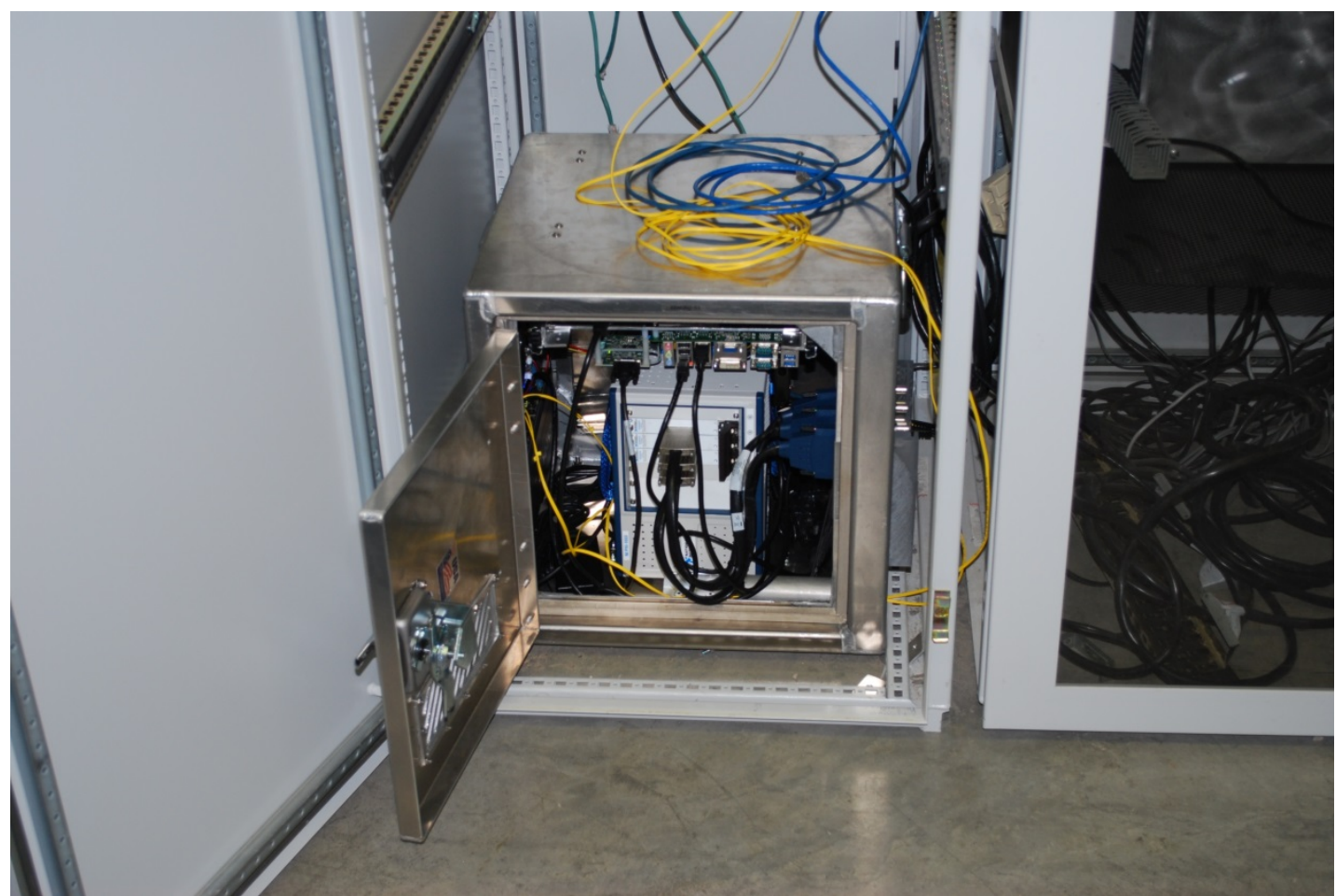

Figure 3. INL typical substation GMD data acquisition system. 
Specific GMD measurement system equipment procured and installed includes the following:

1. Substation data acquisition and recording system units were installed in several INL substations. These systems include Global Positioning System-based time synching across the network of devices for accurate time-stamping of the data.

2. Data from the substations is collected and stored locally on each distributed unit and periodically aggregated to a centralized redundant array of independent disks database server.

3. A below ground, 3-Axis Geomagnetic Field Disturbance sensor system is used to measure and record the magnitude and direction of changes in the earth's B-Field.

4. 0-30A and 0-300A GIC sensors were installed on each of the INL substation transformers neutral/ground current connections that are subject to GIC impact.

5. Data analysis software and algorithms were developed to process and analyze the large volumes of data collected over time for 16 separate measurements on power transformers (approximately 1 terabyte of data for 2 weeks of measurements). Excel Visual Basic was used to create smaller data sets by summarizing data from very small time increments ( 0.05 seconds) and to view the waveforms and graphs that are critical to understanding the measurements. The spreadsheet includes metrics such as average, minimum/maximum, root mean square, Fourier series coefficients, goodness of Fourier curve fit, etc. that can then be used to postulate "trigger metrics" to detect abnormalities in the data. The data was then decimated to $25 \%$ of its original size using the SANDAQ SANView tool, which is MATLAB-based. This reduction maintained sufficient sample size to yield meaningful analysis results while reducing the overall analysis time.

6. Metrics developed for detailed analysis serve to establish baselines with which triggers can be set to detect waveform abnormalities, events, and changes in direct current of harmonic content due to GIC. 
Figure 5 shows $100 \mathrm{~A}$ and $300 \mathrm{~A}$ sensor measurements for a transformer at the INL Test Area North substation.

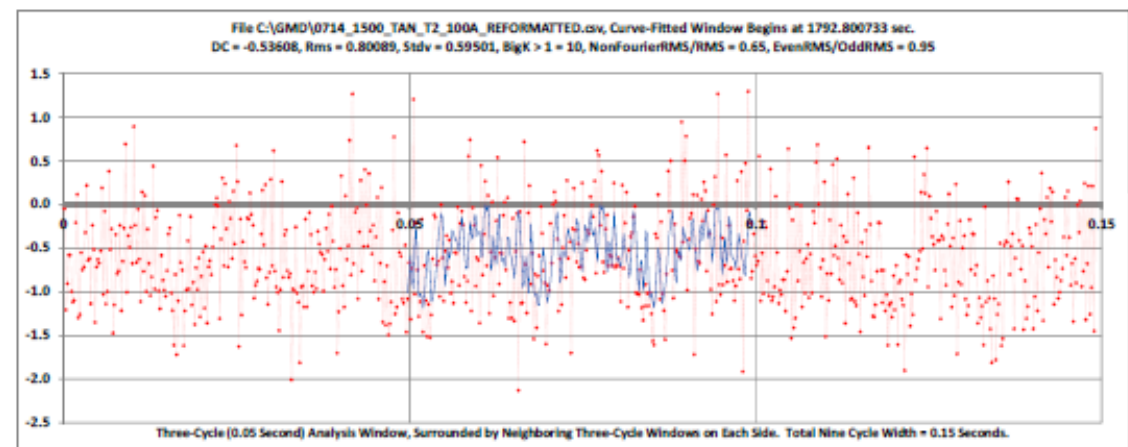
\begin{tabular}{|l|} 
July $14,15: 00$ \\
TAN, T2, 100A \\
DC $=-0.54 \mathrm{~A}$ \\
BigK $=10^{\text {th }}$ \\
NonF $/ \mathrm{F}=0.65$ \\
Even $/$ Odd $=0.95$ \\
\hline
\end{tabular}

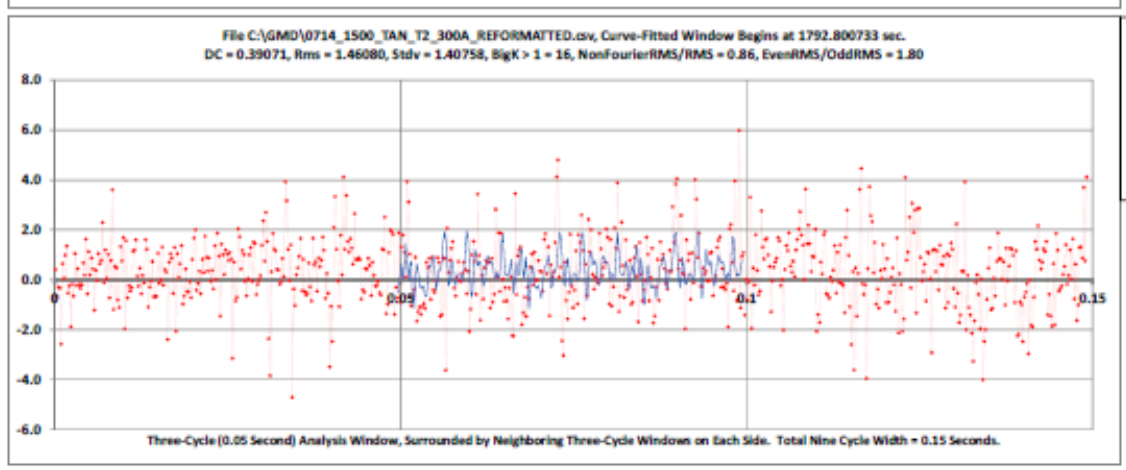

Figure 5. 100A and 300A sensor measurements.

Figure 6 shows average direct current values for 72,000 three-cycle windows in 1 hour as small red dots and a 1-minute moving average shown as the black line.

One can imagine a visual display of time plots of the "black lines" for all key indicators developed from three-cycle windows (e.g., Average, RMS, even/odd harmonic ratio, Fourier content) and so on.

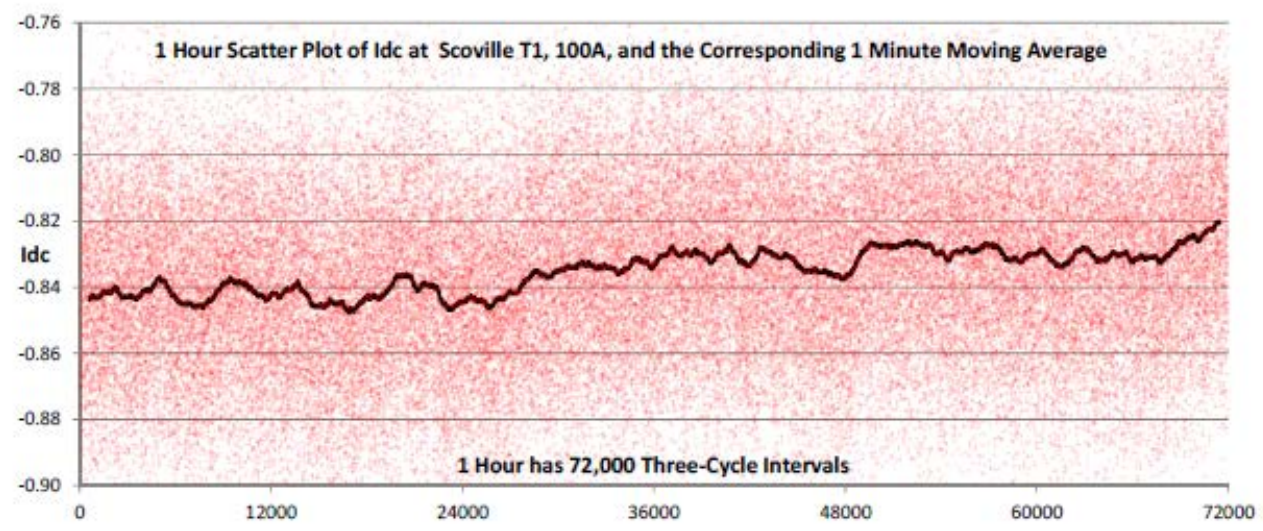

Figure 6. Direct current values. 
Figure 7 further refines the data results to show fast and slow trends.

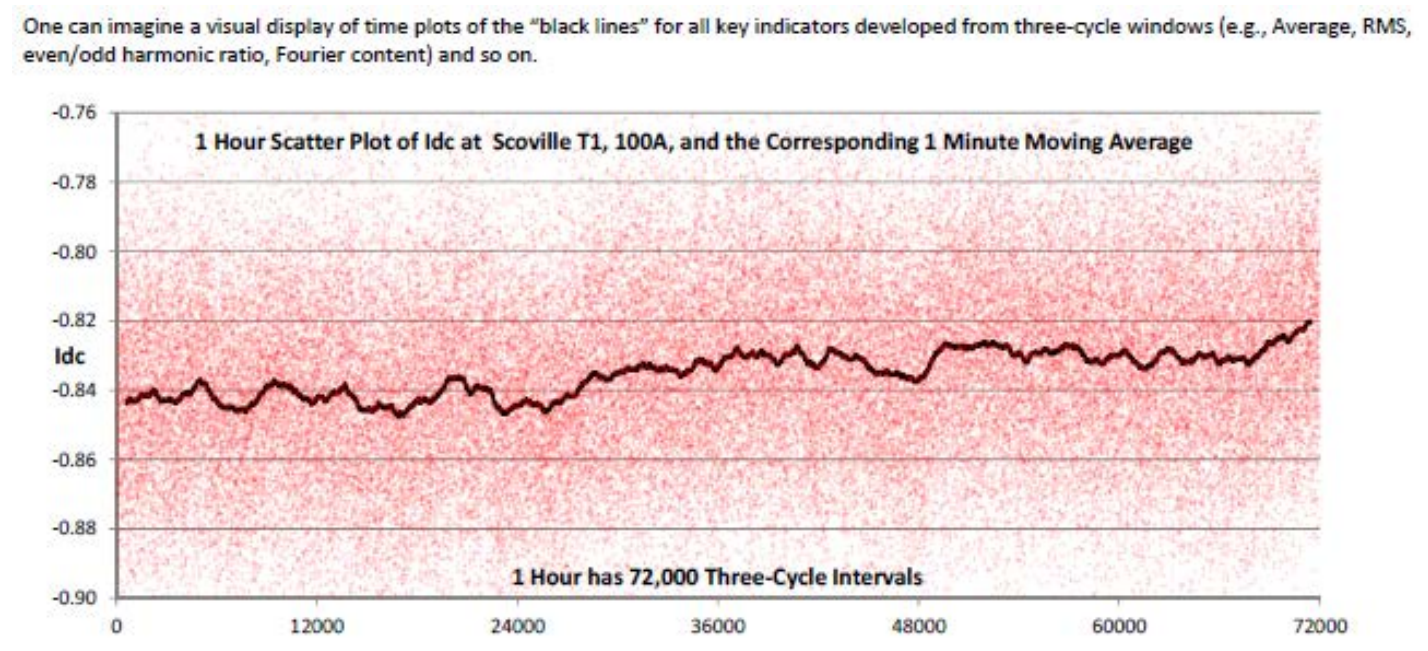

Figure 7. Refined data showing trends.

\section{Benefits to DOE}

The resulting technologies of this project will benefit national security by enhancing the reliability and resiliency of energy supplies for our nation's domestic and foreign military installations. This LDRD is developing analysis products that will be deployable to power utilities to assess solar storm impacts to utility systems for critical infrastructure protection. The resulting technology will significantly advance the ability to monitor the electric power grid with benefit to both Microgrid and Smart-Grid research and implementation activities that rely on sensor-based feedback to increase system reliability, efficiency, and resiliency.

\section{Publications}

West, S., S. McBride, and A. Walker, "INL GMD Measurement System," Report to Industry, in press.

\section{Presentations}

West, S., and A. Walker, "GMD GIC INL DTRA SANDAQ," GMD Conference, Idaho Falls, ID, August 2013. 


\title{
14-032 - Remote Vulnerability Analysis of CAN Bus Networks
}

\author{
Jonathan Chugg and Kenneth Rohde
}

The Controller Area Network Bus (CAN Bus) protocol—developed during the early 1980s, first released in 1987 , and used in a small number of passenger cars-controls the CAN networks in a modern automobile that may contain 50 or more electronic control units (ECU). These electronic control units support a number of different subsystems, including control modules for the engine, powertrain, transmission, antilock braking, airbags, power steering, cruise control, door locks, windows, audio system, battery, and charging system. The CAN protocol also supports many different protocols that are used in a wide variety of areas including automotive, road transportation, rail transport, industrial automation, power generation, maritime, military vehicles, aviation, and medical devices. This research is being performed to discover new technologies capable of enhancing the cybersecurity of the CAN Bus networks that are used within a typical passenger vehicle, with applications that can also address transportation systems related to aerospace, rail, and maritime.

\section{Summary}

This year's research focused on accomplishing two goals. The first was to discover if there is an external cyber vulnerability exposure created by exploiting the wireless communications link used in the Tire Pressure Monitoring System (TPMS) to the core CAN Bus network of a modern vehicle. INL research is emphasizing the discovery and subsequent solutions to complex attack vectors that have yet to be publicly disclosed. The second goal was to identify the possibility of circumventing CAN gateways (modules that shift information between different CAN networks) to migrate from one CAN network to another. Using an attack initiated on a sub-CAN network, research is intended to examine whether an attacker can gain access through CAN gateways to more critical systems. The first year of this LDRD developed laboratory capabilities that included a vehicle test prototype (Figure 1), which required:

- Development of software to decode Tire Pressure Monitoring System signals using a Software Defined Radio

- Completion of initial proof-of-concept CAN message spoofing

- Procurement of a modern test vehicle CAN Bus system

- Development of a prototype hardware/software device to monitor and "fingerprint" CAN networks

- Procurement of test vehicle reprogramming and diagnostics tools.

This vehicle test prototype consists of the electronics of a salvaged vehicle fastened to a display board along with a CAN Bus prototype device that the research team is continuing to develop. This module will be used as a basis for the development of a CAN Bus experimental environment to monitor up to three networks and provide alerts on abnormalities. Initial experiments to migrate from one CAN network to another have determined that CAN gateways only allow a limited set of messages to transition across other CAN networks. 


\section{NATIONAL \& HOMELAND SECURITY}

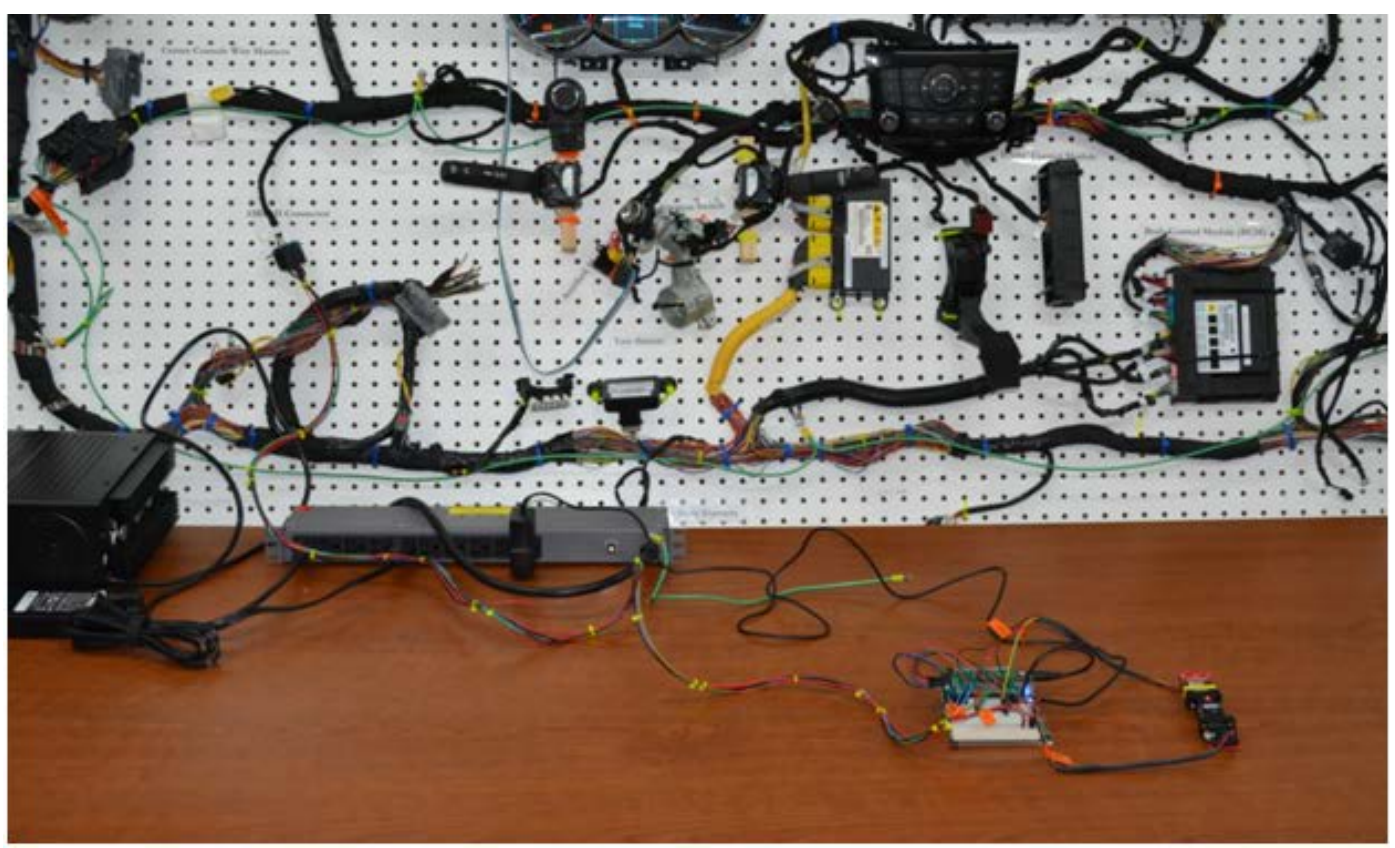

Figure 1. CAN Bus prototype environment.

During subsequent years of this LDRD, the INL research team will continue to conduct proof-of-concept experimentation to explore new emerging technologies employed in CAN devices across the many transportation sectors. Continuation research also will include experimentation with new remote wireless capabilities that will soon be employed in the transportation sector (Figure 2).

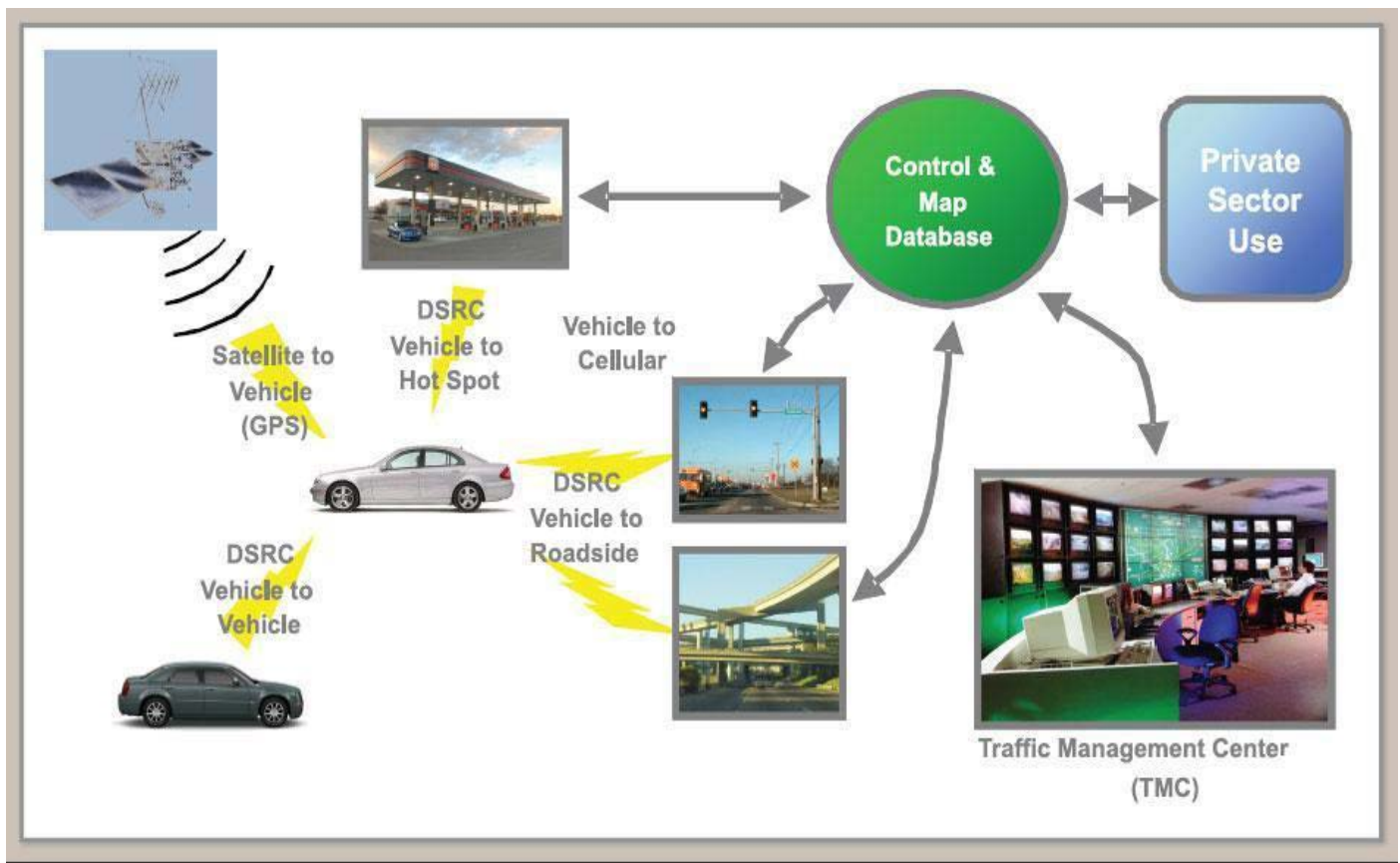

Figure 2. Overview of future vehicle to vehicle and vehicle to infrastructure concepts. 


\section{Benefits to DOE}

The research being performed will enhance the cyber security of all sectors of the nation's critical infrastructure that utilize CAN Bus networks. This research, initially focused on a typical passenger vehicle, is intended to result in deployed cybersecurity innovations that are used in all CAN Bus networks.

\section{Publications and Presentations}

Twenty-two presentations and demonstrations were given during the first year, but no publications were developed. The FY 2015 deliverables include one publication at a relevant conference or journal. Below is a summary of notable FY 2014 presentations:

Chugg, J., and K. Rohde, Strategic Advisory Committee Poster Session, EIL building at INL, Idaho Falls, ID, June 12, 2014.

Chugg, J., Batelle Energy Alliance (BEA) S\&T Committee, INL, Idaho Falls, ID, July 24, 2014.

Rohde, K., "Current Cyber Security Efforts," USCAR: Grid Interaction Tech Team (GITT), ESL Building at INL, Idaho Falls, ID, August 2014.

Chugg, J., Vulnerability Analysis of CAN Bus Networks, Secretary of Energy (Moniz), INL, Idaho Falls, ID, August 19, 2014.

Chugg, J., Vulnerability Analysis of CAN Bus Networks, National Association of Regulatory Commissioners, INL, Idaho Falls, ID, August 21, 2014.

Chugg, J., Vulnerability Analysis of CAN Bus Networks, Department of Energy-Nuclear Energy (DOE-NE), Germantown, DC, August 25, 2014.

Chugg, J., Vulnerability Analysis of CAN Bus Networks, Wisconsin Energy Institute, INL, Idaho Falls, ID, September 8, 2014. 


\title{
14-035 - Safer Energetics
}

\author{
Michael Daniels, Michelle Pantoya, ${ }^{1}$ and Ron Heaps
}

Our objective is to include an additive (or modify the oxidizer) in composite energetic materials that only allows energetic materials to ignite when they are heated at a specific (high) heating rate. These enhancements to energetic materials will reduce the hazards related to environmental conditions that could cause the energetic materials to ignite from electrostatic discharge (ESD) or when the fuel reaches its melting point during storage or transport. These innovative additives are intended to enable the energetic mixtures to only respond to specific on-command ignition stimuli while preventing initiation of the energetic when exposed to any other stray or unintentional stimuli. Advancements in materials chemistry enable this concept to become more feasible and also create opportunities for innovations in the tune-ability of the effectiveness of composite energetic materials.

\section{Summary}

Our approach was to start with a patent-pending mixture developed for its increased safety to ESD stimuli. The mixture is composed of micron scale aluminum (Al) particles combined with copper oxide (CuO) particles and ESD inhibiting materials. This mixture was shown to be desensitized to ESD ignition and also have negligible effect on the mixture's overall combustion behavior. To further develop a safer material, we experimented with a series of additives to seek energetic material mixtures that would be inert when subjected to slow heating processes (i.e., simulating an accidental fire). Through experimentation with various compositions of materials (Figures 1 and 2), we have made significant progress developing a new methodology to tailor a thermite reaction for safer use during multiple applications. Specifically, this research identified an electrical conductivity regime that promoted ESD insensitivity (i.e., $\sim 0.01 \mathrm{~S} / \mathrm{cm}$ ) and explored several additives to desensitize compositions to prevent high energy evolution during the slow heating conditions simulating an accidental fire. We identified the mechanism for neutralizing the formulation through deactivating thermal decomposition and rendering the mixture too fuel rich to achieve sustained propagation. These material discoveries will be further examined under other heating rate (i.e., bake) conditions to better simulate accidental fires representative of a typical hydrocarbon flame, as demonstrated by Babrauska and Peacock in Fire Safety Journal.

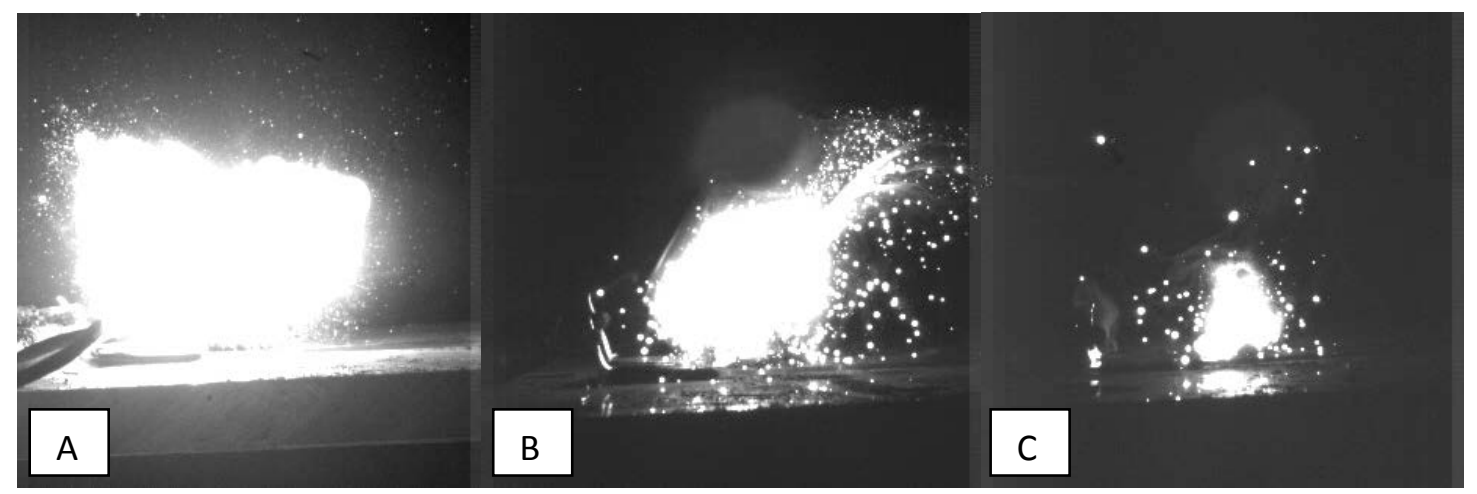

Figure 1. Representative still-frame images of reactions of multiple mixtures of $\mathrm{Al}, \mathrm{CuO}$, and experimental additives.

\footnotetext{
${ }^{1}$ Texas Tech University
} 


\section{NATIONAL \& HOMELAND SECURITY}

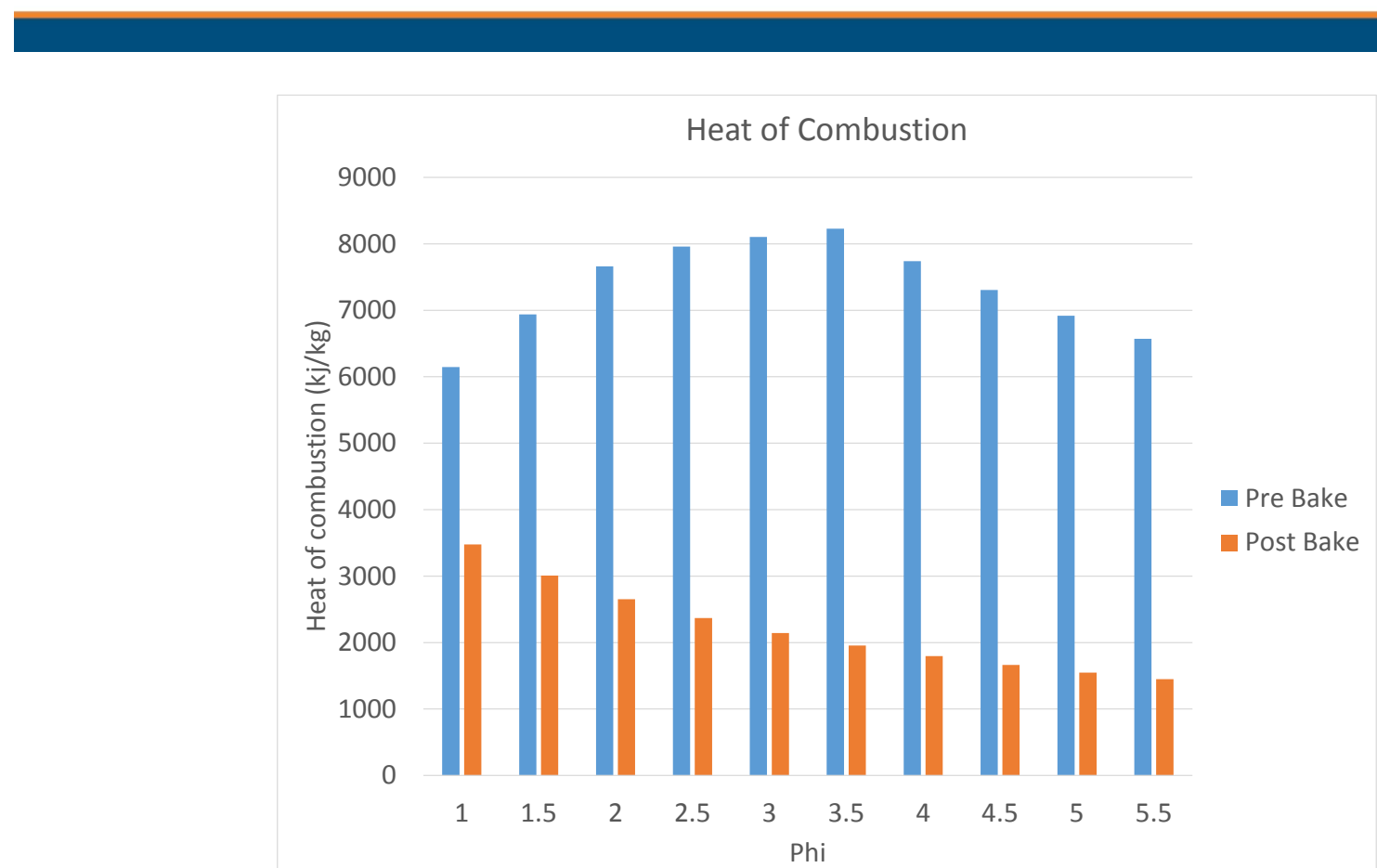

Figure 2. REAL code simulations for the reaction shown with varying material compositions for heat of combustion.

\section{Benefits to DOE}

This project has the potential to greatly improve the safety of personnel in DOE, the Department of Defense, and the Department of Homeland Security who routinely use energetic materials for physical security, emergency response, and material disposition. These novel materials also present the potential for improving the safe use, shipping, and storage of energetic materials in applications associated with infrastructure construction, mining, and first responder emergencies.

\section{Publications}

Steelman, R., M. Daniels, and M. Pantoya, "The Influence of Particle Size on the Electrostatic Discharge Desensitization to Ignition," Journal of Applied Physics, to be submitted, 2015.

Bellow, M., K. Poper, M. Daniels, and M. Pantoya, "Controlling Accidental Fire Ignition Safety of a Thermite with Ammonium Nitrate Additive," ACS Sustainable Chemistry and Engineering, to be submitted, 2015.

Collins, E., B. Skelton, M. Pantoya, F. Irin, M. Green, and M. Daniels, "Ignition Sensitivity and Electrical Conductivity of a Composite Energetic Material with Conductive Nanofillers," Combustion and Flame, May 2014, in review.

Poper, K., E. Collins, M. Pantoya, and M. Daniels, "Controlling the Electrostatic Discharge Ignition Sensitivity of Composite Energetic Materials Using Carbon Nanotube Additives," Journal of Electrostatics, Vol. 72, 428-432, 2014. 


\section{4-036 - Innovative Research for Fieldable Nuclear Measurements}

David L. Chichester, James T. Johnson, Mathew T. Kinlaw, Scott J. Thompson, and Scott M. Watson

This project seeks to evaluate the potential of integrating recent developments in the field of radiation measurement instrumentation with advances in the development of low-power, high-performance miniature computers to implement transformational advances for in-the-field nuclear measurements for emergency first responders and safeguards inspectors. These advances include new concepts for portable instrumentation for use in monitoring radiation fields, such as would be needed for securing a facility as part of a force-protection envelope, and new approaches for the monitoring needed to search and respond to nuclear and radiological emergencies. Also of great interest in this area are emerging concepts and enabling technology related to wearable computing.

\section{Summary}

During the first year of this project, we have focused our research activities in four areas. The goal of our work this year was to explore and characterize key technology enablers that will be exploited in the second year of the project when we will develop prototype fieldable nuclear measurement systems. Our exploration areas included:

(1) exploring methods for using scintillating fiber optic sensors under field conditions; (2) examining the use of next generation, internet-protocol based electronic systems for powering and using gas-filled radiation detectors; (3) examining technology solutions suitable for deploying very light weight, energy-efficient radiation detectors; and (4) expanding capabilities for programming hand-held computer devices, such as smart phones and tablets, to interface with detector instrumentation while simultaneously logging measurement results with phone/tablet metadata, such as global positing system coordinates.

For our exploration into scintillating fiber optic sensors, we built and tested the sub-components that will be needed to deploy a radiation sensor for testing during a field trial for a nuclear security application. This novel prototype sensor system, planned for testing during FY 2015, is comprised of a detector, a data acquisition system, and the supporting electronics for control and real-time data analysis.

In evaluating gas-filled detectors, research done in collaboration with Quaesta Instruments (QI) is demonstrating the utility of the QI Neutron Pulse Module (NPM) for operating a helium (He)-3-based proportional tube for thermal neutron detection. A photograph of the QI NPM used in this study, attached to a polyethylene-moderated He-3 proportional tube, is shown in Figure 1. Also shown in this figure is a standard, analogue electronics unit that is frequently used when operating this type of detector; this analogue system was used to establish a baseline against which to compare the QI NPM. The QI NPM proved to be an exceptionally useful tool for operating gas-filled proportional tubes, with the unit capable of being controlled via an Ethernet link. This capability allows the user to not only verify discrete pulse-shape waveforms (a useful tool for troubleshooting) but also allows the user to dynamically vary the detector's gain and discrimination setpoints. 


\section{NATIONAL \& HOMELAND SECURITY}
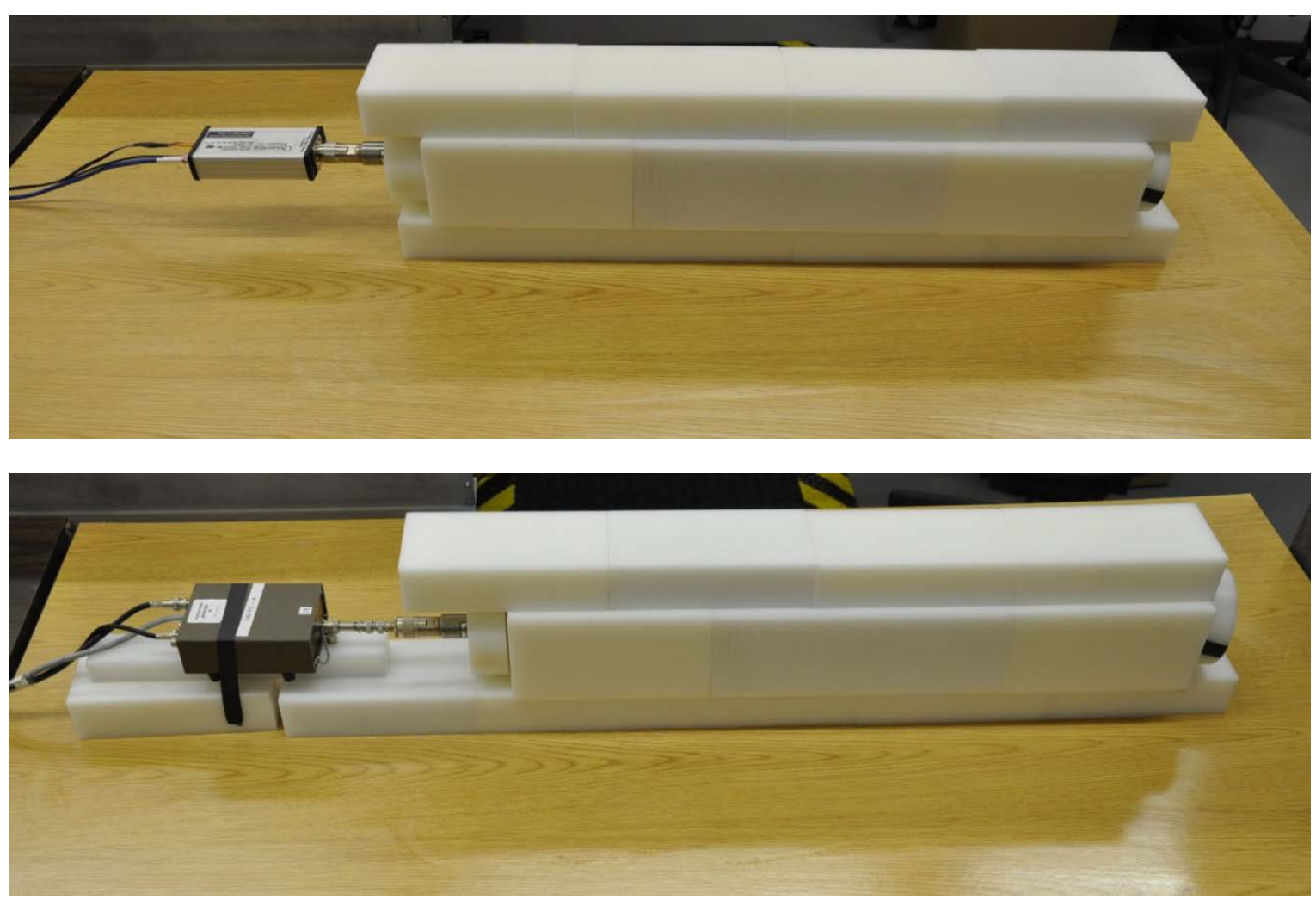

Figure 1. The top figure shows the test configuration used for evaluating the QI NPM when attached to a He-3 proportional tube. The lower figure shows the reference analogue electronic system attached to the same He-3 tube for benchmarking the QI NPM performance.

We assembled and tested a lightweight bench top sensor system that incorporates a scintillator-based gamma-ray detector, a miniaturized data acquisition system, a miniature computer, Bluetooth connectivity, and a power supply. This prototype was demonstrated at a lab-scale to have the capabilities to record and transmit data in a list-mode format that is well suited for field applications. The system weighs less than $1 \mathrm{~kg}$ and serves as an ideal starting point for follow-on research planned for deploying a lightweight, low-power consumption detector system.

In pursuit of this project's fourth topic of research, we have developed an Android-based smart phone/tablet application that allows for the simultaneous logging of radiation detector data and global positioning system data. In addition, we have developed a new data analysis algorithm for interpreting these data to map local radiation fields and locate radioactive sources.

\section{Benefits to DOE}

This project provides benefit to DOE in support of furthering DOE's leadership role in the science and engineering of advanced nuclear detection technology that supports the next-generation of nuclear power through the development of new diagnostic tools and capabilities for use in onsite protection; monitoring radiation levels in and around domestic and international nuclear facilities, nuclear safeguards, radiological safety, and dosimetry; and environmental radiation monitoring. The technology explored in this project may also find use for future used fuel transportation and storage and facilitate new concepts for safe and secure interim spent fuel storage. 


\title{
14-037 - Advanced Nuclear Material Characterization Technology for Security Applications
}

\author{
David L. Chichester, James T. Johnson, Mathew T. Kinlaw, Scott J. Thompson, and Scott M. Watson \\ Emerging nuclear nonproliferation, counterproliferation, and forensic challenges facing the United States require \\ new technological developments in the area of radiation measurement. Often, research programs addressing \\ these areas focus on one type of sensor and one particular observable. It is our hypothesis that the integrated use \\ of orthogonal radiation measurement concepts for detecting and analyzing observables will improve our ability to \\ characterize these materials versus separate uses of these technologies. This project is exploring new sensor \\ combinations that can be used in support of national objectives for detecting nuclear and radiological materials.
}

\section{Summary}

Our primary activity for this project in FY 2014 was to conduct experiments within INL's zero-power physics reactor facility to explore the potential for integrating traditional active neutron interrogation (ANI) signatures from special nuclear material (SNM) with time-correlated neutron analysis methods. Traditional ANI signatures (such as prompt-neutron die-away analysis, delayed neutron analysis, and delayed gamma-ray analysis) have each been shown useful as diagnostic methods for detecting shielded SNM or for verifying the absence of SNM within inspected items. However, comparatively less work has taken place to explore the use of these measurement techniques for characterizing assemblies of shielded SNM to determine attributes such as SNM mass, the composition of the SNM, or the configuration of the SNM. In contrast, time-correlated neutron analysis methods such as thermal-neutron coincidence counting and thermal-neutron multiplicity counting are proven measurement techniques for characterizing SNM.

Experiments were performed at the zero-power physics reactor using an array of neutron detector modules specially designed at INL for use in ANI along with a newly-acquired list-mode data acquisition system. This new data acquisition system is capable of individually recording the relative time of arrival of neutron detection events in the array in relation to an arbitrary reference time (for passive measurements and for interrogation using steady-state neutron sources) or in relation to synchronization time stamps from a pulsed electronic neutron generator. Measurements were performed using varying configurations of INL's MARVEL test assembly of metallic, highly enriched uranium (HEU). The maximum HEU mass used in these trials was $14.35 \mathrm{~kg}$. The material was used bare, reflected with $5-\mathrm{cm}$ of polyethylene, and reflected with $5-\mathrm{cm}$ of tungsten (W). Neutron multiplication levels ranged from $1.8(5.49 \mathrm{~kg}$ of HEU, bare) to 8.8 (14.35 kg of HEU, reflected with $5-\mathrm{cm}$ of polyethylene). A photograph of the configuration for the lowest multiplication level is shown in Figure 1. Work is now underway to analyze and interpret the data generated during this experiment campaign. 


\section{NATIONAL \& HOMELAND SECURITY}

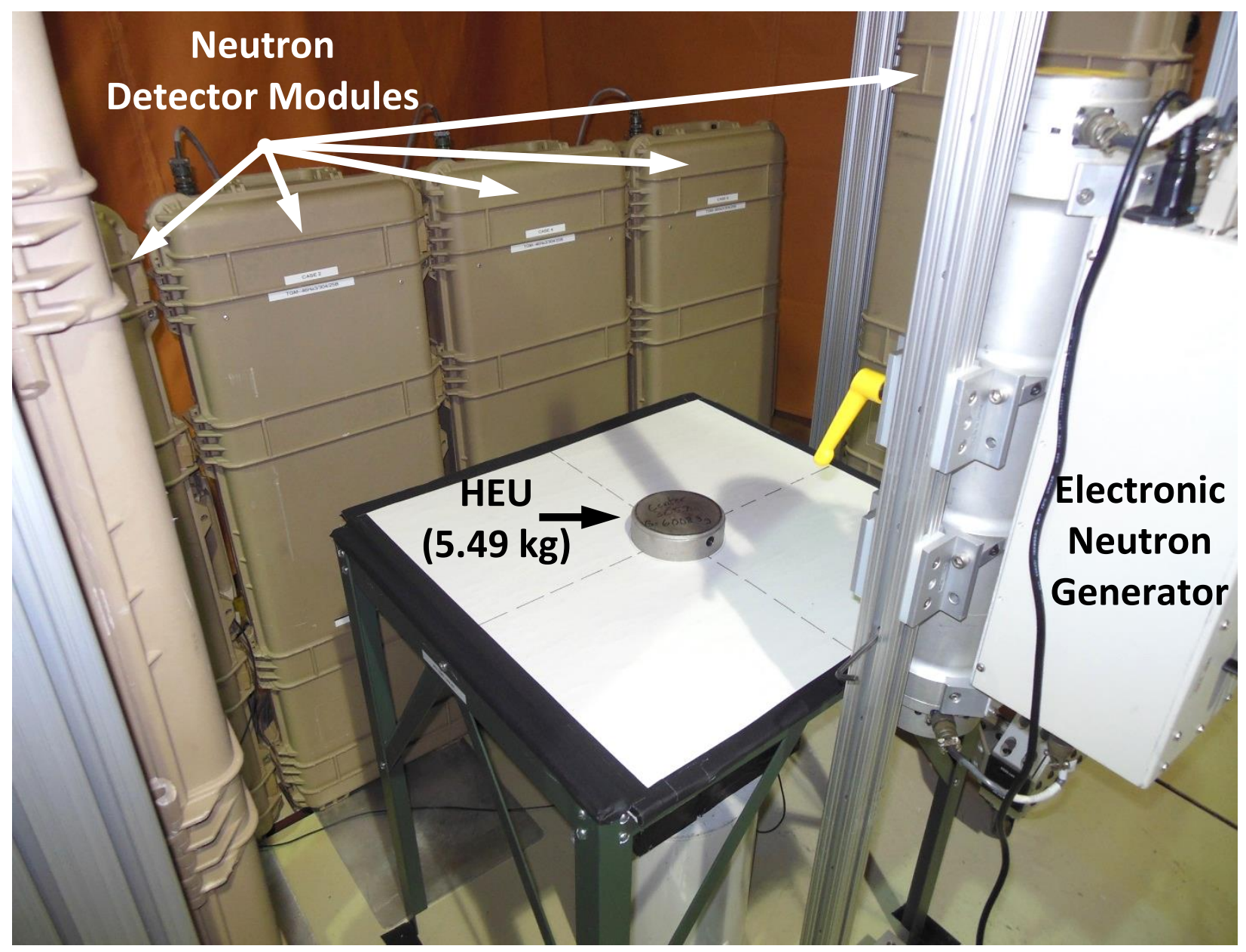

Figure 1. Photograph of an experiment configuration studied at INL's zero-power physics reactor facility to examine the integration of traditional ANI signatures, such as prompt neutron die-away analysis and delayed-neutron analysis, with time-correlated neutron multiplicity analysis.

\section{Benefits to DOE}

This project provides benefit to DOE by advancing the science and engineering principles for the development of advanced diagnostic instruments and methods capable of characterizing shielded assemblies of SNM, in particular HEU. New characterization capabilities that are being developed in this project have relevance across a number of DOE focus areas, including criticality safety, used-fuel storage, nuclear facility operations, nuclear nonproliferation, safeguards, arms control, and treaty verification.

\section{Publications}

Chichester, D., S. Thompson, M. Kinlaw, J. Johnson, J. Dolan, M. Flaska, and S. Pozzi, "Statistical Estimation of the Performance of a Fast-Neutron Multiplicity System for Nuclear Material Accountancy," Nucl. Inst. Meth. Phys. Rev. A, available online October 6, 2014, in press. 


\section{NATIONAL \& HOMELAND SECURITY}

\section{Presentations}

Chichester, D., M. Kinlaw, S. Watson, J. Kalter, E. Miller, and W. Noonan, "Experiments and Simulations of the Use of Time-Correlated Thermal Neutron Counting to Determine the Multiplication of an Assembly of Highly Enriched Uranium," Institute of Electrical and Electronics Engineers Nuclear Science Symposium, Seattle, WA, November 2014.

Chichester, D., S. Thompson, M. Kinlaw, J.Johnson, J. Dolan, M. Flaska, and S. Pozzi, "Statistical Estimation of the Performance of a Fast-Neutron Multiplicity System for Nuclear Material Accountancy," 2014 Symposium on Radiation Measurements and Applications (SORMA XV), Ann Arbor, MI, June 9-12, 2014. 


\section{4-042 - Treaty Relevant Modeling}

\section{Mathew T. Kinlaw}

The Treaty Relevant Modeling project is intended to advance modeling and simulation capabilities supporting nonproliferation/counterproliferation research, development, and testing within INL's Nuclear and Radiological Activity Center (NRAC). The overall enhancements to the current capabilities are expected to have direct impact on the detection and characterization of special nuclear materials and the identification and characterization of proliferation signatures and observables. Further, the research provides a predictive capability to better inform potential and/or planned experimental activities and measurements as well as provide a validation and verification tool for measured data sets.

The primary deliverable of the LDRD was a set of precise, high-fidelity MCNP models representing a series of INL Inspection Objects (IOs), which are briefly described in Table 1. These IOs were designed and constructed to serve as standards against which candidate technologies could be tested to assess their efficacy in performing treaty verification and dismantlement-type measurements. Each IO constitutes a unique combination of materials, containing one or more of the following: fissile material, depleted uranium (DU), polyethylene, lithium hydride, and/or tungsten. These IOs are maintained at INL and are included as part of the NRAC resources made available for experiments, training classes, and response exercises [1]. A general description, with additional details regarding the original design and composition of six IOs, can be found in Ref. [2]. In the process of developing the models, every reasonable effort was made to ensure they represented the as-built items. We recognize that for many applications, approximations or generalizations regarding the modeled geometries and compositions may suffice. Nevertheless, the MCNP models were constructed on a piece-by-piece basis, allowing for greater flexibility with potential future applications. Each IO model also includes a unique set of surface, cell, and transformation numbers, while a consistent material definition list was maintained between all the models. Hence, any combination of the 10 models can be readily integrated into a single input file.

\section{Table 1. General description of the IOs.}

\begin{tabular}{|c|c|}
\hline Item & General Description \\
\hline IO5 & Composite Shielding of HEU: $4.7 \mathrm{~kg}$ of HEU surrounded by $6.4 \mathrm{~kg}$ of DU and $11.0 \mathrm{~kg}$ of $\mathrm{LiH}$ \\
\hline IO6 & Composite Shielding of DU: $5.1 \mathrm{~kg}$ of DU surrounded by $5.7 \mathrm{~kg}$ of W and $11.0 \mathrm{~kg}$ of LiH \\
\hline 107 & DU Shielding of HEU: $5.1 \mathrm{~kg}$ of HEU surrounded by $17.9 \mathrm{~kg}$ of DU \\
\hline 108 & DU Shielding of Plutonium: $1.3 \mathrm{~kg}$ of Pu surrounded by $5.6 \mathrm{~kg}$ of DU \\
\hline 109 & HEU Shielding of Plutonium: $1.6 \mathrm{~kg}$ of Pu surrounded by $4.8 \mathrm{~kg}$ of HEU \\
\hline IO10 & Composite Shielding of Plutonium: $1.3 \mathrm{~kg}$ of Pu surrounded by $5.6 \mathrm{~kg}$ of DU and $5.4 \mathrm{~kg}$ of polyethylene \\
\hline
\end{tabular}


During model development, some attributes of the actual IOs were identified, such as shifting of fuel plates or omission of selected pieces due to complexities experienced during the construction of the actual items. These attributes have been addressed in the models for cases where potential inconsistencies between simulated and measured data are anticipated. As an illustrative example, Figure 1 presents a photograph of interior components of IO9 (left) along with a visual representation of the model geometry (right). In this case, some misalignments and voids between plates exist. These subtle geometry characteristics may be of little consequence for applications based on techniques such as gross counting or standard spectroscopy, but could have greater relevance for alternative applications (radiography, multiplicity analysis, etc.). Figure 2 illustrates the geometry models for each 10 .

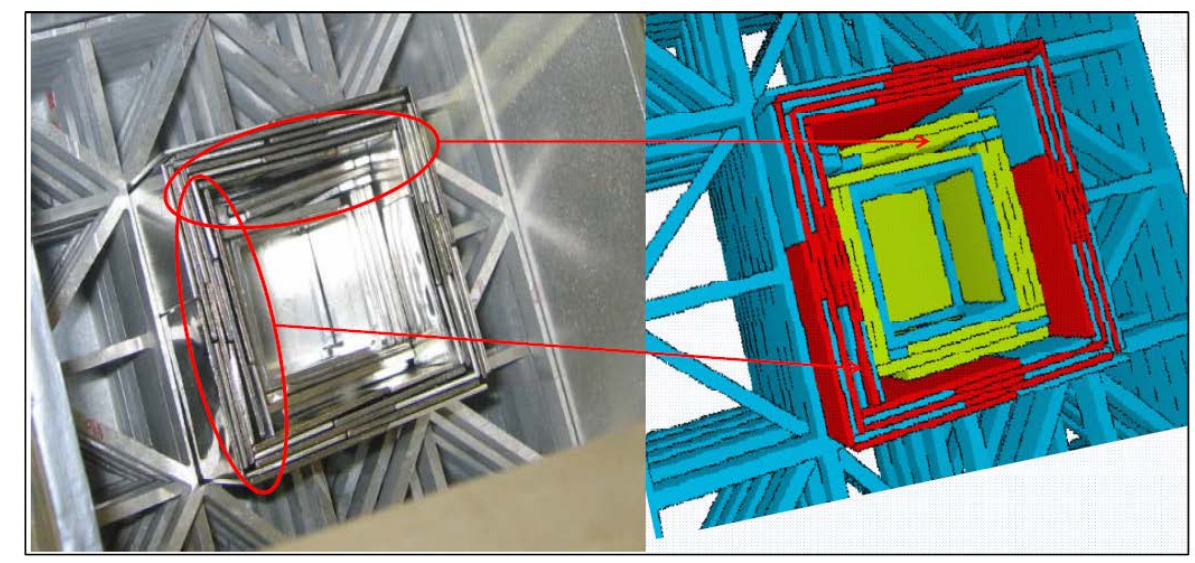

Figure 1. Photograph of interior components of 109 (left), along with a visual representation of the model geometry (right). The circled areas indicate misalignments or shifting of components in the as-built item. When possible, these attributes were included in the model geometries. 


\section{NATIONAL \& HOMELAND SECURITY}

105: Composite shielding of HEU
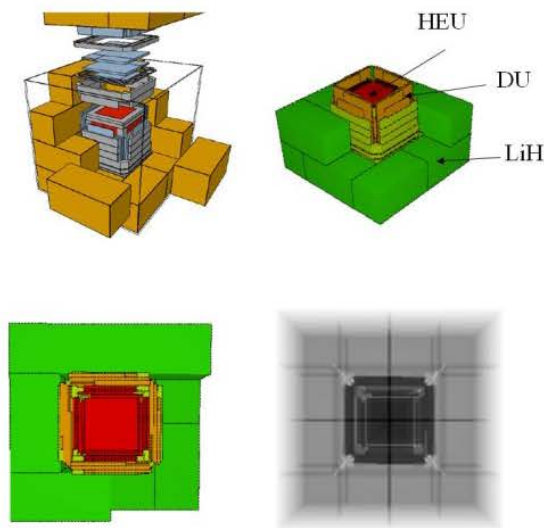

IO7: DU shielding of HEU
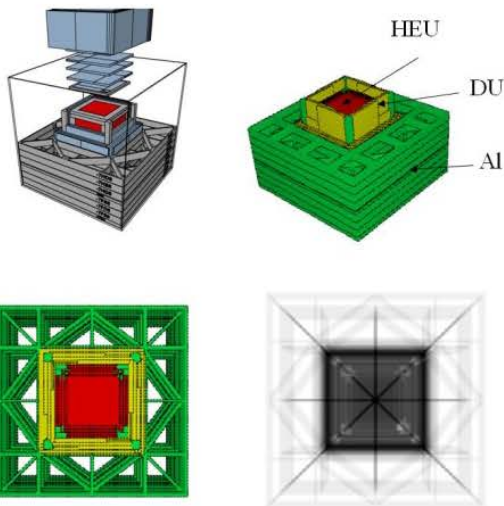

109: HEU shielding of Plutonium
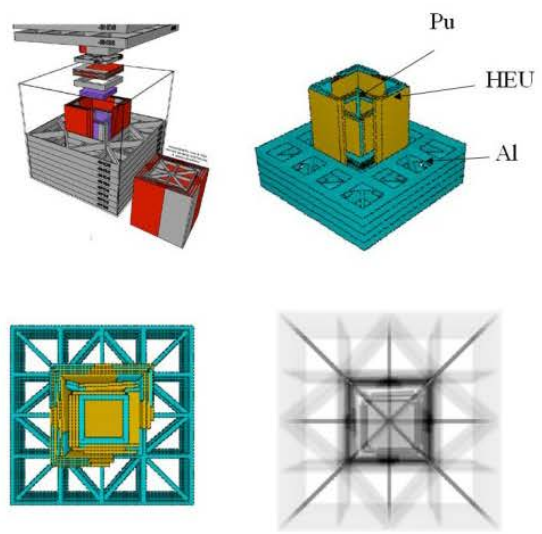

106: Composite shielding of DU
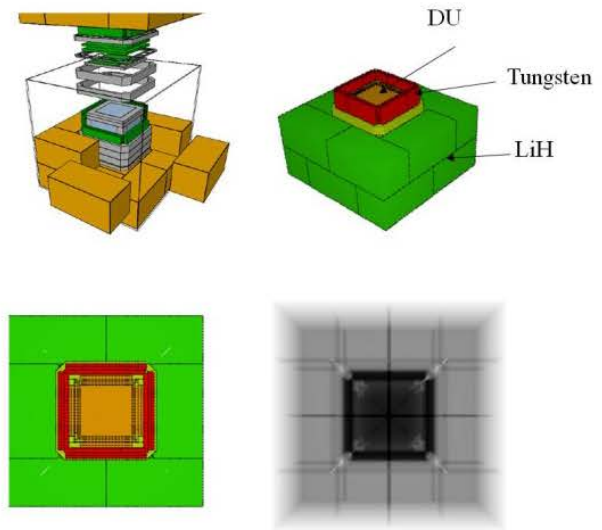

108: DU shielding of Plutonium
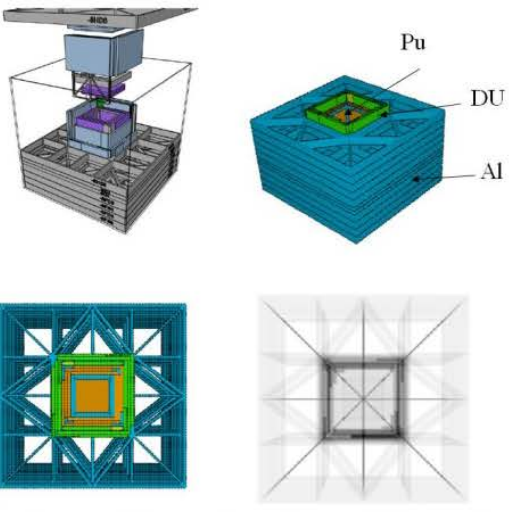

IO10: Composite shielding of Plutonium
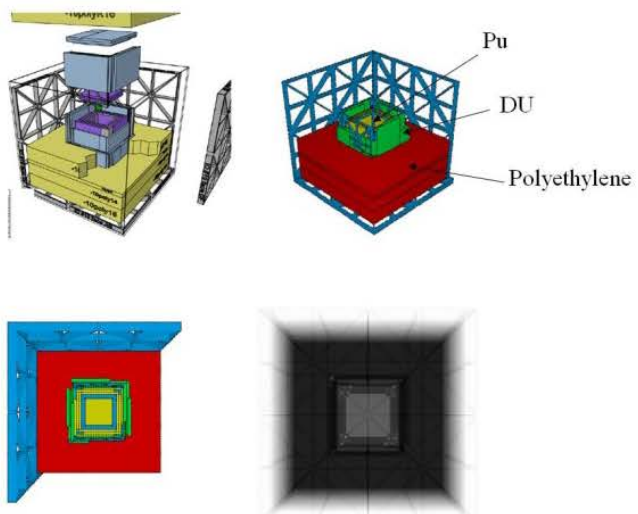

Figure 2. For each IO, the upper left image is an expanded view of the original CAD design taken from Ref. [2]. The upper right shows visual representations of the model geometries at selected steps in the construction processes. The lower left images are top-down views of the model geometries, corresponding to the steps shown in the upper right. The lower right images are simulated radiographs, looking down at the respective IOs. 


\section{Benefits to DOE}

The validation and predictive capabilities provided by this research will have direct application to DOE's national security mission objectives by promoting international nuclear safety and advancing nuclear non-proliferation. The more imperative contribution to current and future research is not merely the possession of models representing the IOs, but rather, the benefit is in the development of high-fidelity models, which have been validated against measurements and will be made readily available for inclusion in initial development and/or testing activities of conceptual, new, or even existing technologies for nonproliferation applications. Many potential shortfalls or challenges can be identified and resolved prior to the execution of the activities and/or physical measurements. This not only provides a benefit in a scientific and economic sense, but also in terms of safety, particularly when developing increasingly challenging detection scenarios for nonproliferation, safeguards, and/or emergency response.

\section{Presentations}

Kinlaw, M., "Treaty Relevant Modeling," presented to the Strategic Advisory Committee, Idaho National Laboratory, Idaho Falls, ID, June 2014.

\section{References}

[1] Chichester, D., et al., "The INL Nuclear and Radiological Activity Center," INL/CON-13-28220, June 2013.

[2] Neibert, R., et al., Passive and Active Radiation Measurements Capability at the INL Zero Power Physics Reactor (ZPPR) Facility, INL/EXT-11-20876, December 2011. 


\title{
14-044 - Development of Halide and Oxy-Halides for Isotopic Separations
}

\author{
Leigh R. Martin, Aaron Johnson, Jana Pfeiffer, and Martha Finck
}

The goal of this project was to synthesize a volatile form of neptunium $(\mathrm{Np})$ to improve mass spectrometry measurements of nuclear materials and fission products for nuclear fuel development, safeguards accountability, and nonproliferation treaty verification. Typically, volatile actinides are in the form of halides (e.g., UF 6 ); however, fluorine $(F)$ is highly corrosive to the sensitive internal components of a mass spectrometer. Since iodide is both mono-isotopic and volatile, an iodine (I)-based Np species, if it can be synthesized, presents a suitable alternative for introduction into the mass separator. To accomplish this goal, the technical work in the project sought to establish a novel synthetic route for the conversion $\mathrm{NpO}_{2}{ }^{+}$(dissolved in nitric acid) to $\mathrm{Npl}_{3}$ and $\mathrm{Npl}_{4}$.

\section{Summary}

To attain a volatile $\mathrm{Np}$ iodide species, the initial experimental objectives of this project were to understand how to successfully control the oxidation of $\mathrm{Np}$ in solution to create pure +3 or +4 oxidation states. Even though chemical methods have often been employed to adjust the oxidation state of $\mathrm{Np}$, the desirability of a high-purity material led researchers to evaluate electrochemical methods to control the oxidation state of Np. Electrolytic methods do not require the addition of any special reagents and their selectivity for the specific oxidation state can be precisely controlled through adjustment of the electrolysis potential.

In addition to oxidation state control, through application of Faraday's Law, the total amount of Np in solution can be determined through the electrolysis process (Figure 1).

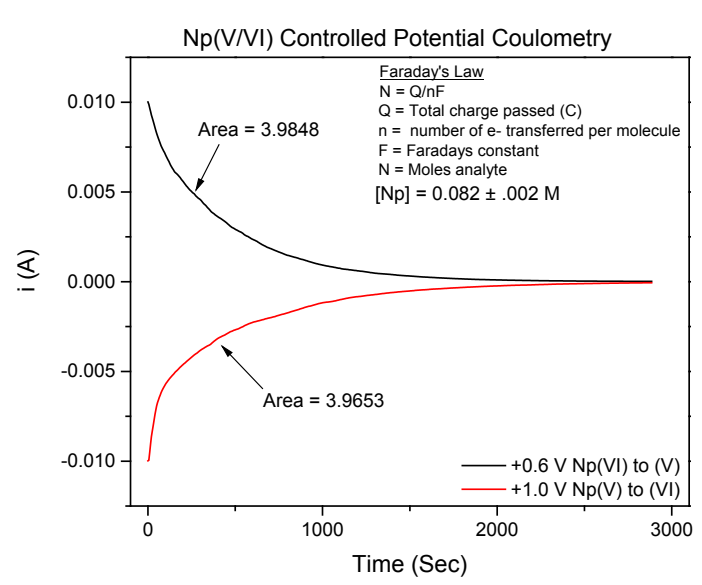

Figure 1. Controlled potential coulometric determination of total [Np]. Np oxidation state controlled in the +5 or +6 oxidation state by applying +0.6 or $+1.0 \mathrm{~V}$, respectively.
Methods to prepare pure amounts of $\mathrm{Np}^{4+}$ and $\mathrm{Np}^{3+}$ via electrolysis were subsequently investigated. The species present in solution post-electrolysis were examined via absorbance spectroscopy and demonstrated that in post-electrolysis, a mixture of $\mathrm{Np}^{3+}$ and $\mathrm{Np}^{4+}$ could be produced with no remaining $\mathrm{NpO}_{2}{ }^{+}$or $\mathrm{NpO}_{2}{ }^{2+}$.

Methods for complete conversion of this $\mathrm{Np}^{3+} / \mathrm{Np}^{4+}$ mixture into their pure components were then examined. After further electrolysis, the $\mathrm{Np}^{3+}$ was quantitatively oxidized to $\mathrm{Np}^{4+}$. These conversion strategies were standardized and verified for synthesis of purified quantities of $\mathrm{Np}^{4+}, \mathrm{NpO}_{2}{ }^{+}$, and $\mathrm{NpO}_{2}{ }^{2+}$ and then confirmed via absorbance spectroscopy (Figure 2). Further experiments determined that pure $\mathrm{Np}^{3+}$ could also be produced from the mixture. However, under these conditions, this reaction only proceeds with the evolution of hydrogen gas, and the resulting $\mathrm{Np}^{3+}$ was unstable in air. Further experiments only proceeded with $\mathrm{Np}^{4+}$ until an inert atmosphere glove box was available. 


\section{NATIONAL \& HOMELAND SECURITY}

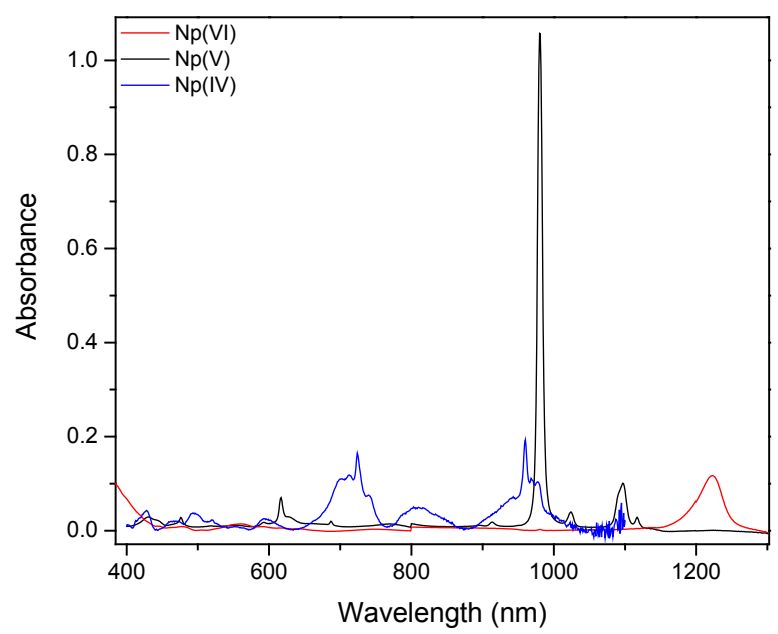

Figure 2. Absorbance spectra displaying purified $\mathrm{Np}^{4+}, \mathrm{NpO}_{2}^{+}$, and $\mathrm{NpO}_{2}^{2+}$.

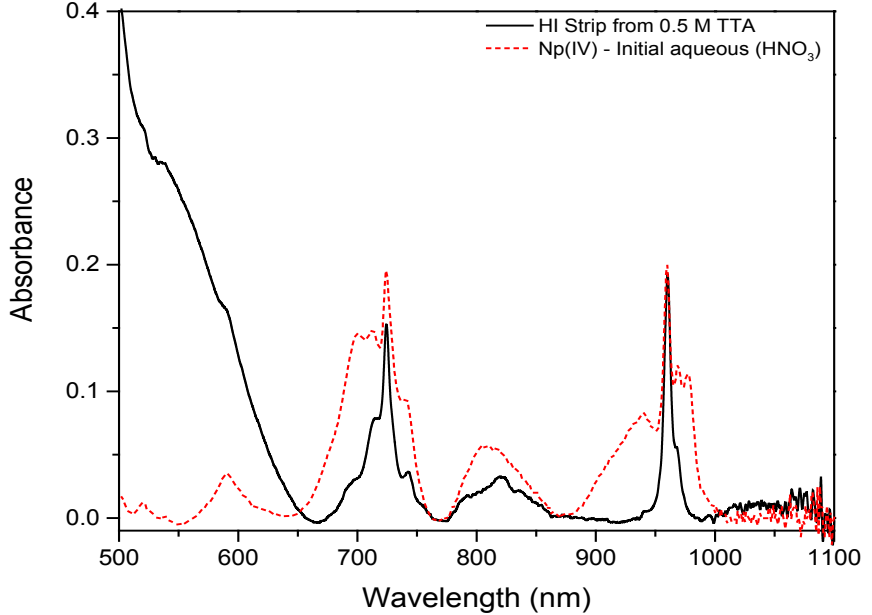

Figure 3. Absorbance spectra of $\mathrm{Np}^{4+}$ in nitric acid before extraction with thenoyltrifluoroacetone and following the strip into purified HI.

Once the electrochemical techniques were understood for the production of $\mathrm{Np}^{4+}$, conversion strategies using solvent extraction methods to create $\mathrm{Npl}_{4}$ were investigated. A method using $\mathrm{Np}^{4+}$ in nitric acid with thenoyltrifluoroacetone in toluene was proven to extract and maintain $\mathrm{Np}$ as $\mathrm{Np}^{4+}$ in the organic phase and then further extracted as $\mathrm{Npl}_{4}$ using hydroiodic acid. The successful formation of a stable iodide compound of $\mathrm{Np}$ was confirmed by absorbance spectroscopy (Figure 3.) This initial confirmation of the successful synthesis of $\mathrm{Npl}_{4}$ has positioned the research to advance to the next experimental phase, forming volatile $\mathrm{Np}$ species suitable for mass spectrometer measurements.

\section{Benefits to DOE}

Developing a reliable synthetic route for the production of volatile $\mathrm{Npl}_{4}$ species will provide a form of $\mathrm{Np}$ suitable for introduction into mass spectrometers for better accountability measurements of nuclear and radiological materials. These improved measurements will increase the nation's confidence in the nonproliferation status of international nuclear energy programs.

\section{Presentations}

Pfeiffer, J., M. Finck, and L. Martin, "Electrochemical Reduction and Subsequent Extraction of Neptunium for $\mathrm{Npl}_{4}$ Production," 38 $8^{\text {th }}$ Actinide Separations Conference, Albuquerque, NM, May 10-14, 2014. 


\title{
14-045 - End-to-End Radiation Detector Enhancements for Improved Safety and Security in Safeguarded Facilities
}

\author{
Scott J. Thompson, David L. Chichester, Daniel Shy, and Mara M. Grinder
}

This project aims to demonstrate that radiation detection systems, such as portal monitors, located at safeguarded facilities can be significantly enhanced through an end-to-end improvement of the scintillation-based detection equipment used within these systems. The use of advanced simulation and modeling techniques in unison with benchmark laboratory testing will allow us to optimize the physical layout and light collection properties associated with the detectors' scintillating crystals for heightened sensitivity to radioactive material. System security can also be enhanced through the application of INL developed data protection methods to transmitted data, reducing the risk of monitor spoofing, data corruption, or eavesdropping. These improvements will not only strengthen efforts to safeguard nuclear materials within facilities, but they also have the potential to enhance the radiation safety measures that protect a site's workforce.

\section{Summary}

The first year of this project focused on the study of how geometrical proprieties affect the sensitivity of organic scintillators. The Geant4 Simulation Toolkit was used to model several different detector form factors. Figure 1 shows examples of the simulated geometries, in which the green lines demonstrate the advanced simulation's capability to track the behavior of light within the detector's scintillation emission regime. During the simulations, each tested geometry was bombarded with cesium (Cs)-137 gamma emissions (662 keV), calculating a detector response for incremental steps in the angle of incidence. The responses were calculated by monitoring the scintillation incident upon the photomultiplier tube window as a function of time and then convolving this distribution with the known characteristics of the tube. The calculated detection pulses were then analyzed statistically to find the minimum pulse shape variance over the range of incident angles. Laboratory experimental measurements to validate the simulation results were made using an isotopic check source (Figure 2).

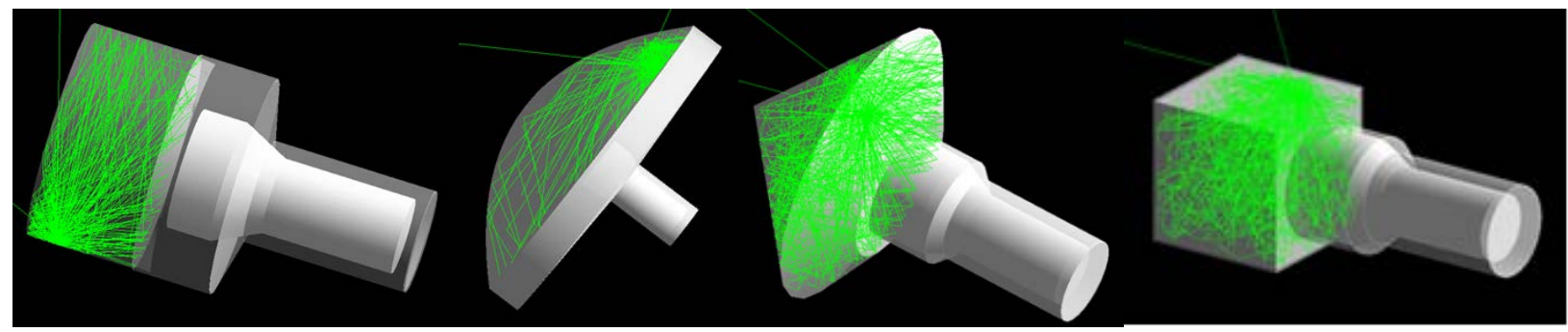

Figure 1. Examples of Geant4 modeled detector geometries; green lines represent the scattering of light within the scintillator. 


\section{NATIONAL \& HOMELAND SECURITY}
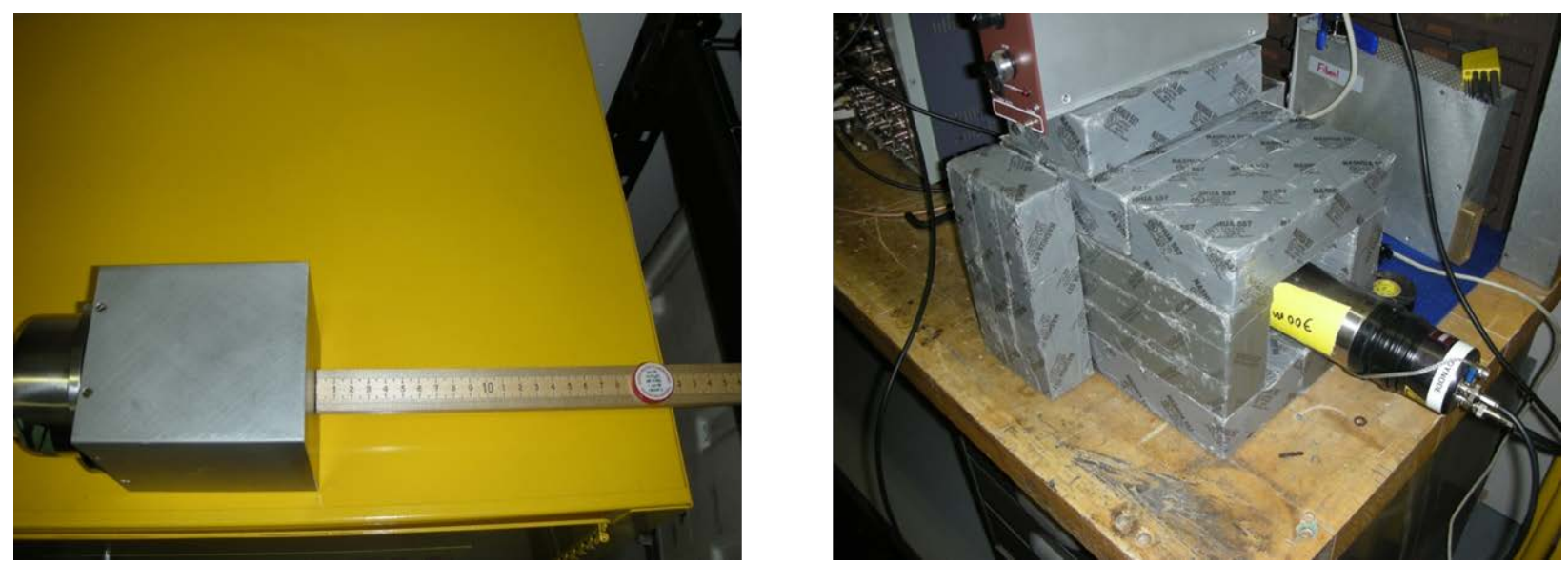

Figure 2. Photographs during model validation experiments of the plastic scintillation models.

\section{Benefits to DOE}

Less expensive high-fidelity sensors will allow for increased confidence in radiation detection for nuclear nonproliferation and counterproliferation measurements related to advanced safeguards, nuclear forensics, emergency response, and nuclear material detection.

\section{Presentations}

Shy, D., "A Parameter Study to Optimize Scintillator Characteristics for Increase Sensitivity in Nuclear Nonproliferation, Safeguards, and Security Based Applications," PNNL Summer Safeguards Course and Symposium, Richland, WA, July 07-August 1, 2014. 


\section{4-087 - Transparent Fiber Reinforcements for Transparent Protection Systems}

Thomas Lillo, Michael Bakas, and Henry Chu

Continuous fiber reinforced composites exhibit considerable increases in strength over non-reinforced materials, resulting in increased performance and an associated reduction in weight. Applications requiring transparent materials have not benefited from continuous fiber reinforcement due to optical transparency requirements. This project endeavors to address this deficiency by developing new transparent fibers of ceramic materials (i.e., aluminum oxynitride [AION] and magnesium aluminum spinel $\left[\mathrm{MgAl}_{2} \mathrm{O}_{4}\right]$ ) with increased strength, general corrosion resistance, and high temperature degradation resistance. These novel materials will allow the development of transparent protection systems with greatly improved performance for government and civilian facilities and vehicles. This project's objective is to evaluate chemical processes to convert non-transparent continuous fibers to transparent continuous fibers for use as reinforcement in transparent protection systems.

\section{Summary}

The experimental approach involved the evaluation of processes to convert a precursor, commercially-available, non-transparent fiber to the desired transparent fiber through high temperature conversion processes. Modeling and experiments evaluated the conversion of fibers to proven transparent armor materials $\mathrm{AlON}$ and $\mathrm{MgAl}_{2} \mathrm{O}_{4}$. Conversion processes were based on the carbothermal reduction of $\mathrm{Al}_{2} \mathrm{O}_{3}$ to produce AION fiber. The modeling results identified the composition of prospective furnace atmospheres that were evaluated experimentally.

Initial experiments with pure nitrogen $\left(\mathrm{N}_{2}\right)$ and $\mathrm{N}_{2}+\mathrm{H}_{2}$ atmospheres did not result in the formation of any AION. Two carbon $(\mathrm{C})$-containing furnace gas mixtures resulted in the uniform deposition of $\mathrm{C}$ onto the fibers with $\mathrm{x}$-ray diffraction results showing the formation of small amounts of AION and AIN. These results indicated AION could be formed by this method, but the conditions were such that AION formation was kinetically limited (not thermodynamically limited), and the formation of AIN was not being suppressed. Ultimately, a two-step conversion process was developed based on these results, each step requiring a different furnace atmosphere, for which a considerable amount of AION was formed (Figure 1.) The results to date are encouraging and immediately suggest a path forward that focuses on changes to the processing time and/or temperature along with relatively slight adjustments to the furnace atmosphere. If complete conversion can be achieved, the researchers will collaborate with commercial vendor(s) of the alumina fiber to assess the commercial interest in producing AION fibers on a commercial scale. 

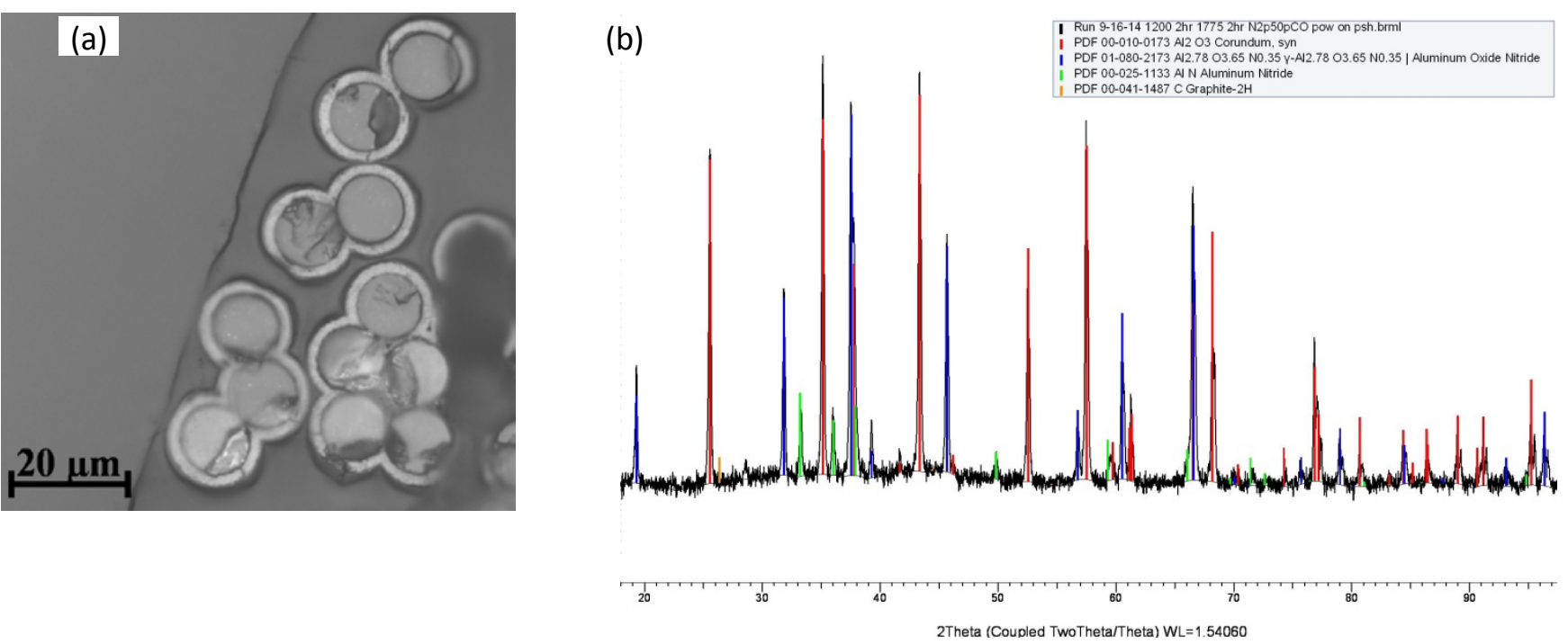

Figure 1. (a) The fibers after a 2-step conversion run. (b) A substantial amount of AION has been produced (indicated by blue lines) along with a small amount of AIN (green lines). The red line corresponds to the remaining $\mathrm{Al}_{2} \mathrm{O}_{3}$.

\section{Benefits to DOE}

To date, this project has made considerable progress toward developing a process to fabricate a new class of optically-transparent fibers that can be used in a variety of transparent protection applications relevant to the security of nuclear facilities as well as various other civilian and law enforcement structures. Furthermore, many military applications will benefit from a new class of transparent fibers. 


\title{
14-090 — SWANS: Secure Wireless Sensor Networks for Smart Grid Communications
}

\author{
Hussein Moradi and Brandon Lo
}

The advent of smart grid technology is manifest in the rapid deployments of smart meters in the nation. The Institute for Electric Efficiency reported in Utility-Scale Smart Meter Deployments: A Foundation for Expanded Grid Benefits that approximately 46 million smart meters were installed nationwide by July 2013 , including installations in about $40 \%$ of U.S. households. The Institute for Electric Efficiency has also predicted that 65 million smart meters will be deployed by the end of 2015 in their report Utility-Scale Smart Meter Deployments, Plans, \& Proposals. These smart meters, along with smart appliances in home area networks and data concentrators in neighborhood area networks, form wireless sensor networks that sense home and neighborhood areas and collect data from households to utilities. However, such rapid deployments pose huge security challenges due to limited protection and security features implemented in these devices. According to B. Krebs in "FBI: Smart Meter Hacks Likely to Spread," the FBI warned that smart meter hacks will likely spread throughout the country because of the ease of intrusion. As a promising technology for secure smart grid communications, wireless systems such as Long Term Evolution provide the benefits of low latency, high throughput, and Quality of Service guarantees to the utilities, as explained in Ericsson's report LTE for Utilities. Moreover, wireless systems' approach to cyber security and easy deployments, just to name a few, are major reasons the utilities, such as Ausgrid, choose wireless technologies for smart grid communications. Nevertheless, recent studies on wireless security, such as Security Attacks against the Availability of LTE Mobility Networks: Overview and Research Directions by R. P. Jover, show that wireless networks are still susceptible to a variety of security attacks.

First, smart grid devices are susceptible to jamming attacks due to the open access of wireless mediums. It is known that jamming can effectively result in Denial of Service. In addition to combating jamming, Secure Wireless interference-Aware sensor Networks for Smart grid communications (SWANS) is effective in mitigating overloading. The emerging machine-to-machine (M2M) communications allow different smart grid devices to autonomously connect through heterogeneous networks available in the smart grid, demonstrated by Z. Fan, R. Haines, and P. Kulkarni in "M2M Communications for E-health and Smart Grid: An Industry and Standard Perspective." However, the traffic and the signaling of a large volume of M2M devices can overload utilities in a smart grid. More importantly, overloading can potentially destabilize or even bring down the grid due to the significant increase of round-trip delay, much longer than the required transfer time of fast messages defined in International Electrochemical Commission 61850 for the reporting of the grid states and substation communications in smart grid. Lastly, since smart grid devices are prone to intrusion, insider attacks, also known as Byzantine attacks, can be launched through these devices to manipulate the grid data or state estimation in the grid when these devices are compromised. Thus, it is crucial to address these wireless security issues. The objective of this LDRD is to provide a novel and solid solution for secure smart grid communications.

\section{Summary}

In this study, we propose the SWANS system for smart grid devices such as smart meters and data concentrators to address wireless security challenges in home and neighborhood area networks. The preliminary SWANS system architecture is devised and completed. Moreover, a variety of wireless security issues such as jamming, overload, and insider attacks in home and neighborhood areas and in Long Term Evolution cellular systems are identified and investigated. 


\section{NATIONAL \& HOMELAND SECURITY}

The SWANS system architecture comprises three components: (1) feature detection and classification for jamming and overload detection; (2) multiband, multiclass, multiuser resource management for jamming and overload mitigation; and (3) a recommendation system for data falsification attack detection. The feature detection and classification solution in SWANS identifies the cyclic features embedded in the jamming signals and classifies those identified features for jamming mitigation. The jamming mitigation is achieved by redirecting the smart grid communications in different frequency bands through SWANS resource management. Thus, SWANS considerably mitigates the harmful interference perceived by smart meters and data concentrators in a smart grid. Furthermore, SWANS effectively detects abnormal traffic pattern changes and latency variations of packet delivery and mitigates overload attacks by rescheduling and rerouting the M2M traffic in a smart grid. Finally, the SWANS recommendation system detects data falsification or data integrity attacks by using a reputation recommender with collaborative filtering. The recommender of the SWANS system efficiently classifies the anomaly features in the collected grid data to first identify the compromised devices used for grid data manipulation. These devices can then be isolated and their reporting data can be excluded from grid data analytics and state estimation. Therefore, SWANS protects the integrity of grid data and the stability of the smart grid.

Dr. Brandon Lo was an intern from Georgia Tech in 2010 , now he is a full-time employee of INL and is a major contributor for developing predictive algorithm in this research.

\section{Benefits to DOE}

This project contributes to DOE's mission by enhancing the security of energy infrastructure and providing a critical component for delivering efficient, reliable, and secure energy generation, transmission, and distribution in a smart grid. Our research conforms to the Energy Independence and Security Act of 2007 (Title XIII) that envisions the smart grid as the modernization of the nation's electricity transmission and distribution systems that are vulnerable to natural disasters and security attacks. Moreover, the project significantly eases the concerns of the device developers and manufacturers for the operational reliability and security of Supervisory Control and Data Acquisition systems, Advanced Metering Infrastructure devices, wireless sensors, and wireless M2M devices in smart grid.

\section{Presentations}

Moradi, H., and B. Lo, "SWANS: Secure Wireless Sensor Networks for Smart Grid Communications," Presentation to Chris Irwin for DOE-OE Visit, Idaho Falls, ID, March 27, 2014.

Lo, B., "SWANS: Secure Wireless Sensor Networks for Smart Grid Communications," SAC Poster Session, Idaho Falls, ID, June 12, 2014.

Lo, B., , "LTE Security in Smart Grid Communications: Challenges and Directions," White Paper, July 2014. 


\section{4-093 - All Hazards Critical Infrastructure Knowledge Framework}

Ryan Hruska, Cherrie Black, Mary Klett, and Milos Manic ${ }^{1}$

The objective of this research project is to develop advanced knowledge discovery and decision support methodologies to improve the nation's capability to conduct infrastructure interdependency analysis to prepare for, protect against, respond to, and recover from all hazard threats to U.S. critical infrastructure. There were two main experimental pathways for this project: the development of a knowledge model framework for critical infrastructure and the development of adaptive hybrid data and expert driven algorithms for online learning of infrastructure characteristics.

\section{Summary}

Technical progress achieved in the development of a knowledge model framework included: (1) the completion of a dependency profile generation for a range of lifeline infrastructures, (2) completion of the evaluation and selection of graph database architecture that integrates capabilities for ingesting structured infrastructure information from numerous open source and government owned databases, and (3) completion of proof-of-concept dependency models and a case study for regional water infrastructure. These developments (Figure 1) have demonstrated the potential of utilizing structured knowledge models to effectively relate and link disparate information sources to produce a holistic representation of critical infrastructure.

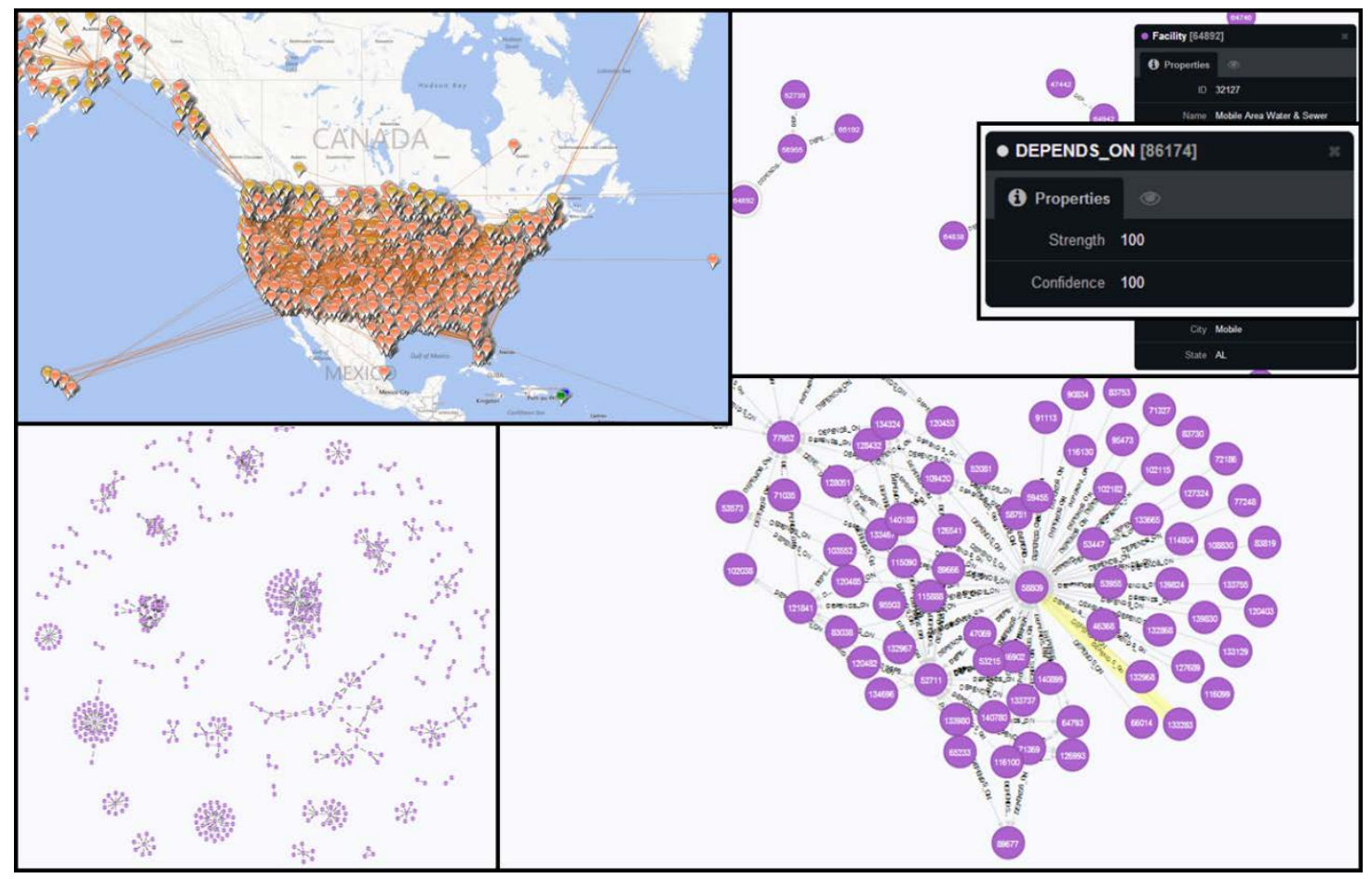

Figure 1. Graph and geospatial dependency models for critical infrastructure.

\footnotetext{
${ }^{1}$ University of Idaho
} 
Technical progress achieved in the development of adaptive data and expert algorithms included:

(1) development of natural language processing techniques for extracting infrastructure information from unstructured text with capabilities to perform name recognition for critical infrastructure facilities and to perform geolocation tagging for spatial information extraction, (2) development of a prototype automated document processing architecture for unstructured text, and (3) development of a prototype graph and geospatial visualization application. These developments demonstrate the capability to transform unstructured text resources into an intuitive spatial context for rapid incorporation into the decision making process.

\section{Benefits to DOE}

This LDRD project is benefiting DOE, Department of Homeland Security, and INL by developing a scientific understanding of advanced knowledge discovery and decision support methodologies to ensure productive, optimal, and secure use of the nation's energy and critical infrastructure resources. The capability developments will lead to enhanced understanding of the complex interdependent relationships within our infrastructure's integrated systems, as well as their vulnerabilities to threats and hazards.

\section{Publications}

Hruska, R., C. Tuchez, and M. Manic, "Extracting Infrastructure from web content using Natural Language Processing," IEEE SSCI 2014, Orlando, FL, December 9-12, 2014, submitted.

Amarasinghe, K., R. Hruska, and M. Manic, "Selection of Optimal Dimensionality for Text Document Content Classification Using Genetic Algorithms," IEEE SSCI 2014, Orlando, FL, December 9-12, 2014, submitted.

\section{Presentations}

Hruska, R. and M. Klett, "Knowledge Framework for Critical Infrastructure Analysis," IEEE Resilience Week, Denver, CO, August 2014. 


\title{
14-094 - SMC Advanced Armor and Materials
}

\author{
Michael Bakas and Henry Chu
}

To be effective as armor, $\mathrm{SiC}$ must be bonded to a support structure or encapsulated in a ductile metal structure that adds considerable weight and volume. Since adhesives are relatively weak compared to other potential joining methods, this project conceived and evaluated methods of joining $\mathrm{SiC}$ to steel by brazing, a process similar to welding. In brazing, a second metal is melted to form a bond between two materials. The technical hurdle is that $\mathrm{SiC}$ does not bond well to most metals, so it is necessary to develop a coating that can serve as an interface between the ceramic and the steel.

This research is focused on developing two different tools to advance ceramic material technologies that can improve performance and reduce weight of armor. To develop lighter weight and higher performing armor systems, it is necessary to develop new enabling material technologies. Hence, this research pursued a novel method to bond ceramic silicon carbide ( $\mathrm{SiC}$ ) to steel and methods for spark plasma sintering (SPS) of aluminum oxynitride (AION), a very hard transparent ceramic.

AION has great potential for improving windows and other transparent armors, but the cost of conversion of AION into a transparent material is expensive. The SPS process utilizes pulsed electrical currents to heat a material very rapidly, which could potentially produce transparent materials at a lower cost than more conventional methods.

\section{Summary}

The first step in developing the SiC-to-steel joining process was to develop a metallic composition that would spread evenly over the SiC but avoid reacting with it in such a way that the joint would be weak. Several different metallic mixtures were attempted with a focus on using iron (Fe)-based compositions, as it was hypothesized that such coatings would have a greater likelihood of being successfully brazed to steel. Once samples were coated, they were sectioned so the interface between the $\mathrm{SiC}$ and the coating could be examined. The initial examination revealed many of the coatings attempted had reacted with the $\mathrm{SiC}$ or did not spread well, but a select few appeared to be successful. To better study the coating/ceramic interface, a focused ion beam attached to a scanning electron microscope was used to section the coating (Figure 1). Using the focused ion beam, it was found that an $\mathrm{Fe}, \mathrm{Si}$, and molybdenum (Mo) mixture coated the SiC well; however, the high Si content of the coating resulted in oxidation on the surface. As this oxidation would interfere with brazing, it was necessary to use a vacuum environment with metal sponges inserted to react with trace oxygen to coat the samples. 
AION is formed by reacting aluminum oxide $\left(\mathrm{Al}_{2} \mathrm{O}_{3}\right)$ with nitrogen $(\mathrm{N})$, often in the form of aluminum nitride (AIN). Several samples were produced, some of which were mixtures of $\mathrm{Al}_{2} \mathrm{O}_{3}$ and $\mathrm{AIN}$ intended to react during the SPS process, others of which were a pre-reacted AION powder. These samples were sintered in the SPS furnace at various temperatures. The AION produced from both groups of samples was very dense but not transparent. Upon examination with a microscope, the samples had formed $\mathrm{N}$-rich phases rather than pure AION. These results indicate that the material was responding to the SPS process much differently than initially hypothesized. After further evaluation of the SPS processes and methods for chemical formation of AION, research will examine the impact of SPS process adjustments on the formation of AION.

\section{Benefits to DOE}

The technologies being developed under this project are all aimed at supporting the DOE security mission with the potential to assist the mission of the Department of

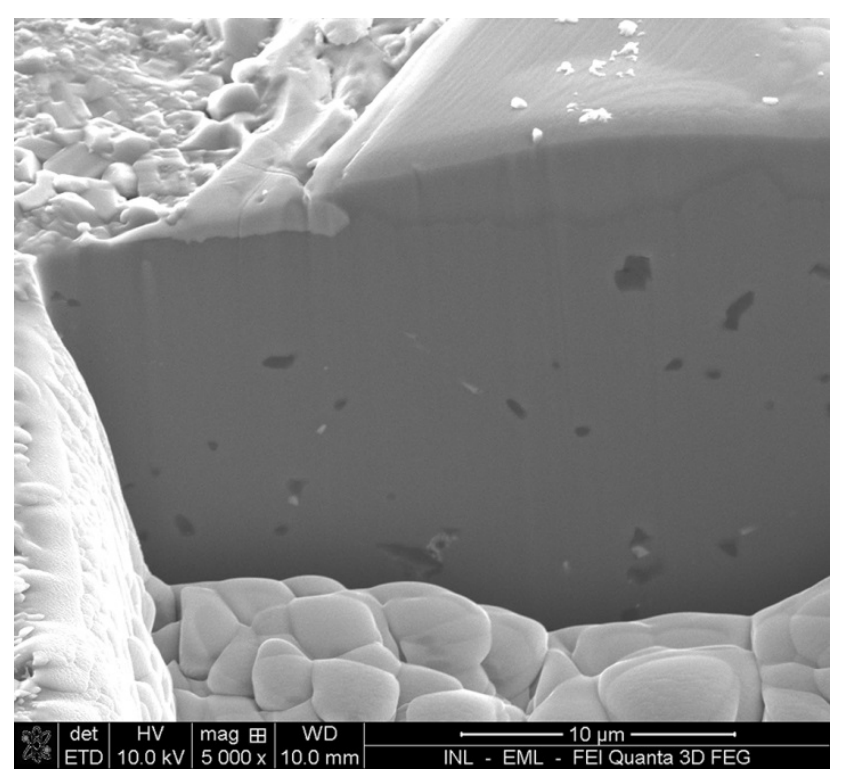

Figure 1. SiC sample coated with a metallic coating which has been sectioned using the focused ion beam, allowing the interface to be examined with minimal damage. Defense, as they can be potentially used in new armor solutions and designs. In addition, the SPS of AION has scientific value even if it does not produce transparent material due to the unusual response of the material to the SPS process. 


\title{
14-103 - Grid-Centric Demonstration of Resilience Technologies
}

\author{
Craig Rieger, Humberto Garcia, Tim McJunkin, and Miles McQueen
}

Presidential Policy Directive 21, "Critical Infrastructure Security and Resilience," recognized the need to advance R\&D for resilient critical infrastructure. At the core of critical infrastructure operation are control systems. This 2-year project was initiated to perform an integrated demonstration of several resilient control system technologies to protect the nation's power grid from natural, operational, or man-made disruptions. This demonstration focused on the R\&D activities needed to apply several innovative INL technologies developed under the Instrumentation, Controls, and Intelligence Systems (ICIS) Distinctive Signature to a common power grid platform.

\section{Summary}

The first year of the project pursued two objectives:

- Completing a demonstration of the experimental platform that included the equipment, connectivity, and software for hosting the ICIS intelligent technologies on a power grid platform

- Completing initial development and integration of the resilient control system technologies for integration with the experimental platform, including translation of algorithms and technology characteristics for the chosen platform environment.

In developing an experimental platform, a MicroGrid (developed under INL LDRD Project 14-086, shown in Figure 1) was chosen and adapted to enable implementation of Known Secure Sensor Measurements; Resilient Battery Monitoring (RBM); Resilient Condition Assessment Monitoring, Adaptation, and Control; and Data Fusion/Visualization technologies. Based on each of the resilience technologies to be demonstrated, the entire Microgrid (or a relevant portion of the Microgrid) was identified, and provisional scenarios were developed to be refined with input from the members of the ICIS external review committee. Known Secure Sensor Measurements is a grid-wide technology used to prevent unobservable attacks to the state estimator of an electric grid through the placement of a secure, encrypted sensor in the form of a phasor measurement unit. Using the topology of the Microgrid and the theoretical framework previously developed, the needed locations for phasor measurement units were determined for each unobservable attack. The grid was emulated in a simulated LabVIEW environment to demonstrate the effects of the integrity attack on the operator display. In cooperation with Idaho State University, phasor measurement units were identified and made available to the project. 


\section{NATIONAL \& HOMELAND SECURITY}

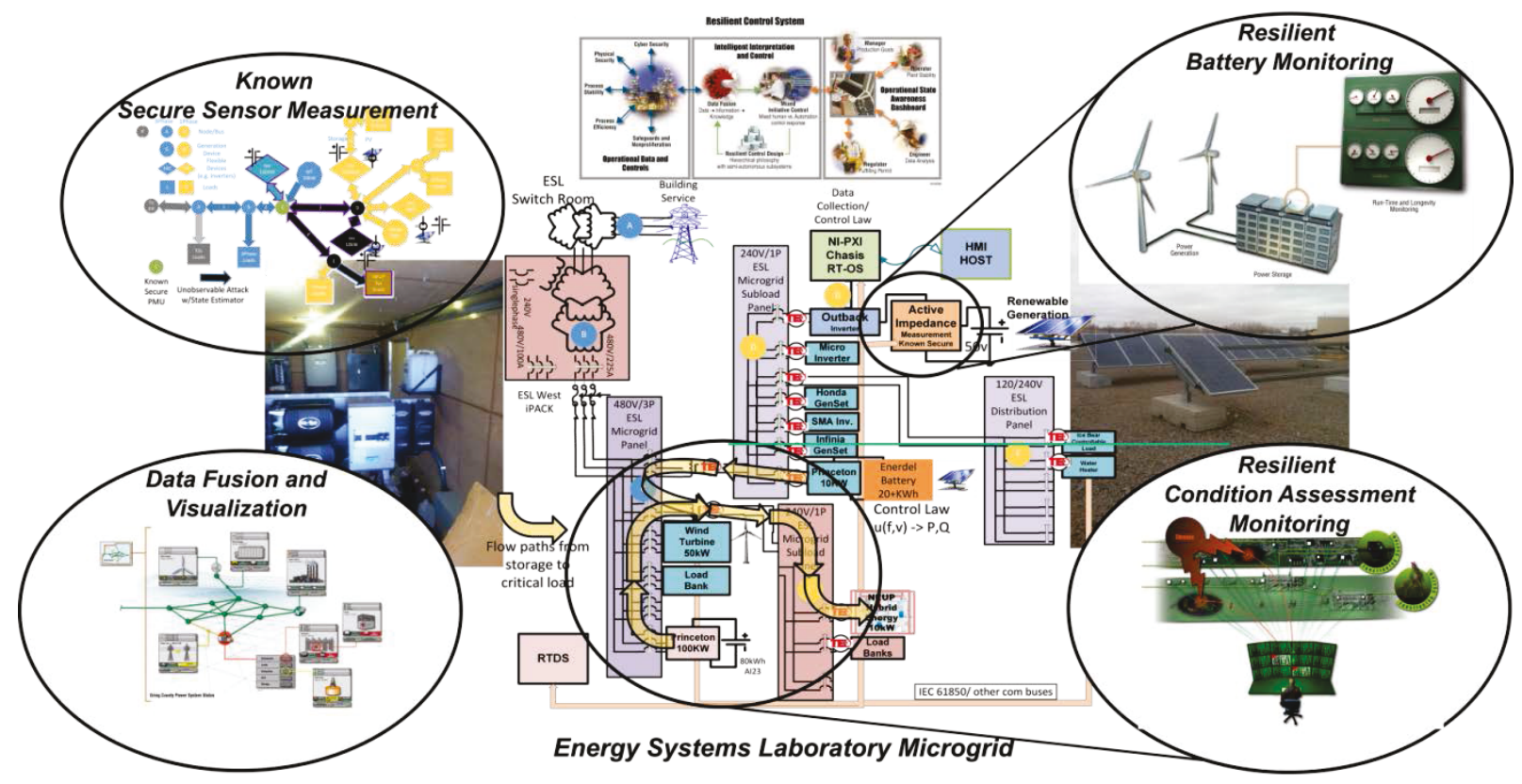

Figure 1. Diagram of the Microgrid and the mapping of resilient control technologies on the platform.

RBM was designed to make use of a previous R\&D 100 award winning technology for active impedance measurement (AIM) of batteries to add resilience to storage systems. The storage systems provide backup, real, and reactive power compensation to stabilize voltage and frequency through a power inverter, which is critical to an extremely low inertia Microgrid. AIM software was enhanced in two ways. First, Montana Tech modified the AIM dynamic linked library to enable integration to the Microgrid control system to take data at regular intervals and/or when understanding the health of the battery is critical. Second, software was developed to take the AIM data from the dynamic linked library and determine the key features of the impedance spectra needed for the RBM methods. Finally, preparations were made to install AIM on the lead (Pb)-acid batteries in the system.

Resilient Condition Assessment Monitoring, Adaptation, and Control provides a Bayesian algorithmic framework for identifying anomalous sensors based upon data quality and using that information for a health determination of the system. For the Microgrid platform, a Bayesian belief network design was completed that uses data quality metrics to ascertain the health of the photovoltaic solar and the batteries (provided from RBM) and perform a diagnostic analysis of physical sensor and cyber security sensors on the power distribution channels.

Data fusion and visualization were developed by the University of Idaho to enable situational awareness to the operator of each of the technologies. Supervisor control and data historian architecture deployment was initiated to provide the structure to implement the four technologies on the Microgrid.

The first year outcomes put the project in position to complete implementation and prepare and execute a demonstration in the second and final year of the project. 


\section{Benefits to DOE}

Resilience is a measureable attribute of a system's ability to successfully maintain defined minimum operational capacity during operational disruptions due to equipment malfunction, human error, and/or deliberate physical or cyber security attack. The development of technologies to improve the U.S. critical infrastructure's capability to perform well under conditions of natural disaster or enemy attack is important to the mission of DOE and the Department of Homeland Security, as well as directly applicable to the infrastructure utilities of the U.S. The ability to demonstrate these technologies provides a direct tie to the ICIS science basis and relevance to how a resilient control system might be formed as an enabler to a Smart Grid.

The project engaged university partners at University of Michigan, University of Idaho, Idaho State University, and Montana Tech. A University of Idaho intern supported this effort.

\section{Publications}

Garcia, H., J. Christophersen, and W. Lin, "On-Line State-of-Charge, State-of-Health, and Remaining-Useful-Life Assessments of Lithium Ion Cells Using Rapid Impedance Spectrum Measurements," in final preparation.

Lin, W., K. Villez, and H. Garcia, "Experimental Validation of a Resilient Monitoring and Control System," Journal of Process Control, Vol. 24, 621-639, May 2014.

Garcia, H., W. Lin, S. Meerkov, and M. Ravichandran, "Resilient Plant Monitoring System: Design, Analysis, and Performance Evaluation," 2013 IEEE 52nd Annual Conference on Decision and Control (CDC), Firenze, Italy, December 2013. 


\section{ENERGY AND ENVIROAOANGE END OTECHNOLOOY}




\title{
12-093 - Development of Unique Nanomaterials for Selective Replacement of Strategic and Critical Materials
}

\author{
Robert V. Fox and Joshua Pak ${ }^{1}$
}

The effort reported here specifically focuses on the synthesis of ternary, quaternary, and doped metal chalcogenide quantum dots, either as individual crystalline nanoparticles or as hybrid and core-shell structures, that are specifically designed to replace rare earth phosphors in solid state lighting (SSL) applications. Highly luminescent semiconductor nanomaterials, having high quantum-yield and stable thermal performance, are ideal for high power and/or high energy-density applications such as those found in light emitting diodes (LEDs), laser diodes, and energy-efficient SSL. The latest research in SSL phosphor technology, demonstrated by Q. Dai, C.E. Duty, and M.Z. Hu in "Semiconductor-Nanocrystals-Based White Light-Emitting Diodes," has shown that metal chalcogenides and doped metal chalcogenides are best suited as rare earth phosphor replacements in all three current white LED commercial designs.

\section{Summary}

During FY 2014, which was the final year of this project, Chalcopyrite, Kesterite, and related crystalline systems were prepared, characterized, and evaluated for their photochemical and photophysical behavior. Additionally, we constructed and tested a number of crude LEDs using synthesized nanoparticles. While quantum efficiency of synthesized crystalline nanoparticle systems could be further improved through thermal treatment and changes in stoichiometry, the peak quantum efficiency of our core-shell materials has not yet achieved the maximum values found in literature. Further investigation indicates that the technique used to form the shell over the core may be undergoing cation exchange between zinc $(\mathrm{Zn})$ in the shell layer and the core. Infusion of $\mathrm{Zn}$ into the core and changes in core stoichiometry or phase may affect quantum yields.

Another route to increasing core-shell quantum efficiency was developed by substituting silver (Ag) with copper $(\mathrm{Cu})$ in the crystalline matrix. Several Ag-containing Chalcopyrite nanoparticles were prepared using both single and multi-precursor methods. AgInZnS ${ }_{3}$ alloy nanoparticles demonstrated the highest quantum efficiency measured to date in this study. In addition, core-shell particles comprised of AgInZnS ${ }_{3}$ cores and $\mathrm{ZnS}$ shells were prepared and deposited into a crude colloidal quantum dot electroluminescence test device. The particles were found to luminesce upon application of voltage to the device. Along with optimizing device construction, new particles were synthesized, characterized, and tested to improve luminescence properties. New chemistry to apply a distinct shell phase was performed. Changing the passivating organics from alkanethiols to amines holds promise for the generation of a distinct core-shell.

A procedure for determining quantum efficiency of nanoparticle solutions using fluorescence was developed. It is similar to a procedure discussed in "Measurement of Fluorescence Quantum Yields" (Allen, Thermo Scientific Technical note 52019) and "Facile Synthesis of ZnS-CulnS2-Alloyed Nanocrystals for a Color-Tunable Fluorchrome and Photocatalyst" (Zhang, Inorg. Chem. 2011, 50, 4065-4072). Briefly, solutions of Rhodamine 590 dye and the nanoparticles are prepared. Solutions are diluted so the ultraviolet-visible absorbance at $450 \mathrm{~nm}$ is equal to approximately 0.1 . Fluorescence excitation curves are obtained for the properly diluted samples with the excitation wavelength set at $450 \mathrm{~nm}$. The excitation curve area is calculated for each

\footnotetext{
${ }^{1}$ Idaho State University
} 
sample. Rhodamine 590 is used as the standard and is assumed to be $96 \%$ efficient. Quantum efficiency can then be calculated using a single point calculation method. Results from these tests will not be shown here but are on file and have been forwarded to the nanoparticle builders.

A circuit to test for electroluminescence of nanoparticles was built. Models for luminescence test-cell construction were taken from the articles "Continuously Voltage-Tunable Electroluminescence from a Monolayer of $\mathrm{ZnS}$ Quantum Dots" (Xuan, Applied Physics Letters 98, 041907, 2011) and "Air-Stable Operation of Transparent, Colloidal Quantum Dot Based LEDs with a Unipolar Device Architecture" (Wood, Nano Lett. 2010, 10, 24-29). A number of methods were also attempted for spin coating the LEDs. CdSe/CdS core shell nanoparticles were purchased from Ocean NanoTech, LLC and used as a reference luminescent material.

Using the commercially prepared nanoparticles shows the cell construction method is working but could be improved. Spin coating parameters could be refined, deposition layer thicknesses could be optimized, and the substrate glass coated with tin ( $\mathrm{Sn}$ ) oxide could be replaced by indium $\mathrm{Sn}$ oxide conductive glass. Different metal oxide layers could also be used instead of $\mathrm{ZnO}$ and $\mathrm{ZnO} / \mathrm{Al}_{2} \mathrm{O}_{3}$.

\section{Dissemination of Results}

Two peer-review manuscripts are being prepared and await final transmission electron microscopy images. The first manuscript covers research progress to date for $\mathrm{CulnS}_{2} / \mathrm{ZnS}$ core-shell particles. The second manuscript covers the $\mathrm{AgInZnS}_{3}$ data and discoveries related to particle performance and luminescence properties.

\section{Benefits to DOE}

Production of semiconductor quantum dot nanomaterials useful for LED applications will allow for substitution of critical materials such as rare earth oxides. Substitutes for critical materials will remove potential materials supply barriers that are preventing commercial, energy-efficient SSL devices from going to market. 


\section{2-113 - Coupled-Smoothed Particle Hydrodynamics and Discrete Element Model (SPH-DEM) Simulation of Hydraulic Fracturing and Shale Gas Production}

Hai Huang and Milind Deo ${ }^{1}$

A better understanding of the coupling between fluid flow, deformation, and fracturing in shale formations could be used to increase the gas recovery factor and more adequately address environmental concerns associated with hydraulic fracturing. The overall objective of this project is to develop a physics-based, multi-dimensional, coupled flow-transport-geomechanics/fracturing computer model based on the combination of two particle-based methods (Smoothed Particle Hydrodynamics [SPH] and the Discrete Element Method [DEM]). The application of the SPH/DEM model can facilitate systematic studies on the nonlinear interactions between fluid flow, proppant transport and settling, and rock failure and deformation at multiple scales, ranging from the core and lab scale to the scale of field hydraulic fractures (hundreds of meters). These studies will fill important gaps in knowledge and fundamental understanding of hydraulic fracturing processes.

\section{Summary}

During the 3 years of the project, a couple of key technical milestones have been met, which include:

- Development of a parallel DEM code based on the Message Passing Interface standard and library, which has been tested and validated on multiple platforms.

- Successful coupling of the DEM code with SPH and the network flow model. The coupled model was applied to a variety of hydraulic fracturing scenarios and generated physics-based hydraulic fracturing patterns that have attracted a lot of attention from industry (see some examples in this report).

- Successfully conducting a laboratory hydraulic fracturing test using a cubic foot granite sample during FY 2013 through a collaboration with Colorado School of Mines The data collected was used to validate the coupled hydraulic fracturing model during FY 2014.

- Extension of the coupled fracturing model to study the dynamics of natural fractures in tight rock formations under hydraulic and thermal stimulations during FY 2014.

Figures 1-4 show the simulated dynamics of hydraulic fractures and natural existing fractures within heterogeneous tight rock formations under various scenarios.

\footnotetext{
${ }^{1}$ University of Utah
} 


\section{ENERGY \& ENVIRONMENT SCIENCE \& TECHNOLOGY}
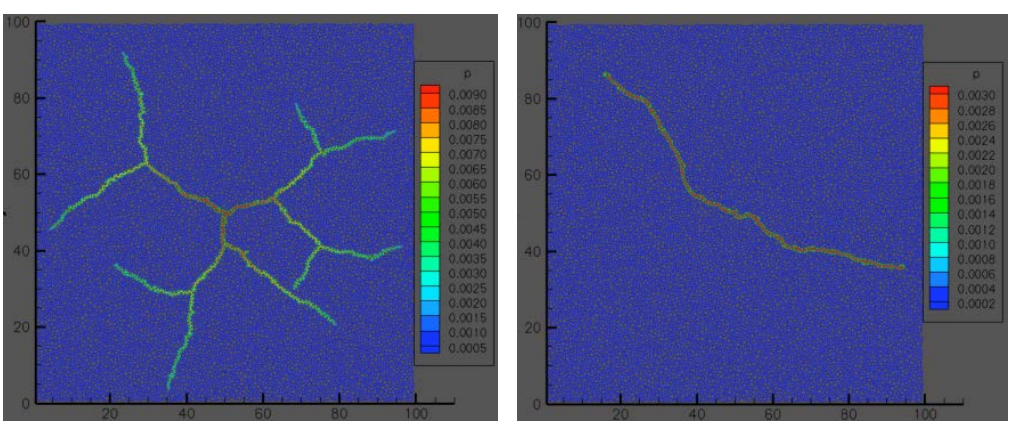

Figure 1. Effect of fluid viscosity on fracturing patterns for a vertical well: (left) high-viscosity and (right) low-viscosity. Color scales with fluid pressure.
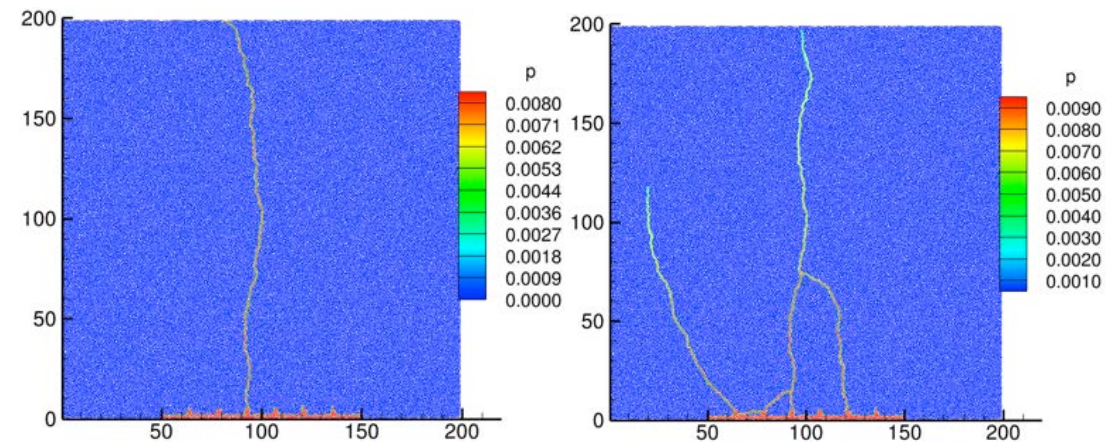

Figure 2. Simulated hydraulic fractures from a multistage horizontal well bore with multiple perforations: (left) low-viscosity fracturing fluid and (right) high-viscosity fracturing fluid. Color scales with fluid pressure.

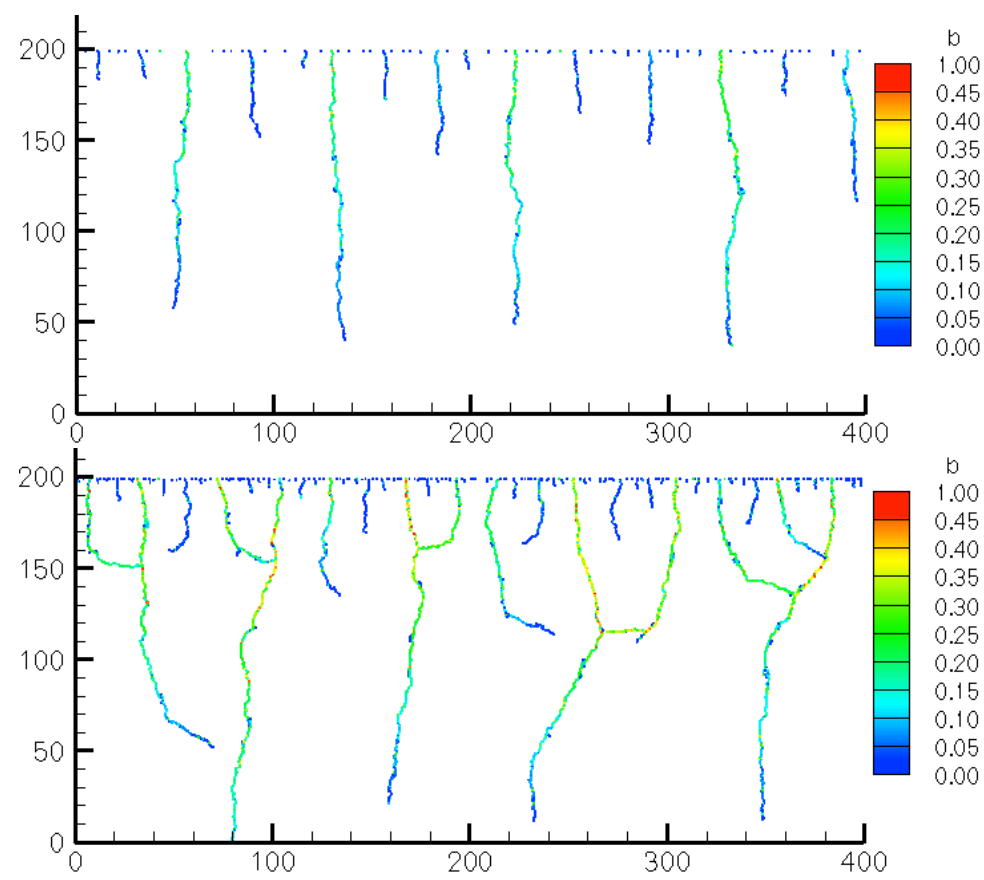

Figure 3. Simulated initiation and growth patterns of hot and dry brittle rocks under thermal cooling: (top) small to mild thermal load and (bottom) large thermal load. Color scales with crack aperture. 


\section{ENERGY \& ENVIRONMENT SCIENCE \& TECHNOLOGY}
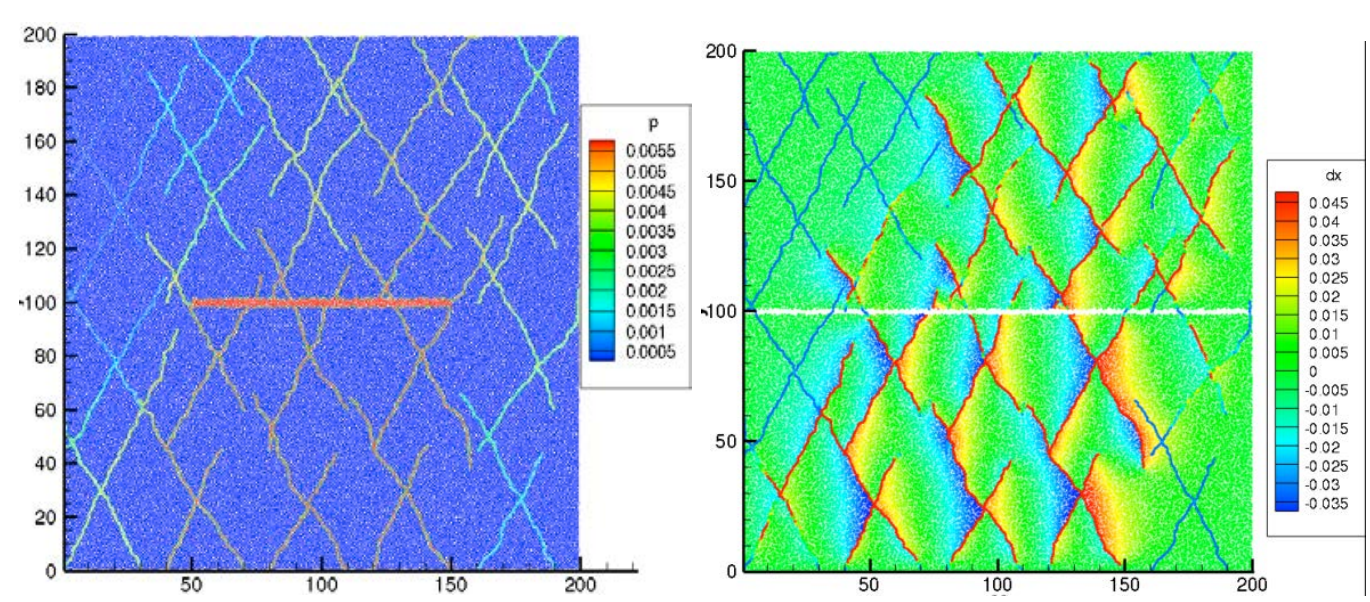

Figure 4. Dynamics of natural fractures in a tight rock formation under hydraulic stimulations: (left) fluid pressure field and (right) horizontal displacement field with fractures colored by fracture permeability.

\section{Benefits to DOE}

The development of the coupled hydraulic fracturing model is of critical importance for the economic and environmentally sustainable production of hydrocarbon fuels from tight rocks (shales), making it directly relevant to DOE's energy security mission. The modeling approach and codes developed in this project will also be readily applicable to a wide range of important subsurface energy/environmental applications at various scales such as enhanced oil and gas recovery from tight source rocks besides shales, thermomechanical fracturing in underground compressed air energy storage, enhanced geothermal system reservoirs, and the geological storage of $\mathrm{CO}_{2}$, chemical waste, and radioactive waste.

\section{Publications}

Huang, H., P. Meakin, and A. Malthe-Sorenssen, "Physics-Based Simulation of Multiple Interacting Crack Growth in Brittle Rocks Driven by Thermal Cooling," International Journal for Numerical and Analytical Methods in Geomechanics, in review.

Huang, H., E. Mattson, and R. Podgorney, "Modeling of Propagations of Interacting Cracks Under Hydraulic Pressure Gradient," World Geothermal Congress, Melbourne, Australia, April 19-24, 2015.

Huang, H., E. Mattson, and R. Podgorney, "Physics-Based Modeling of Hydraulic Fracture Propagation and Permeability Evolution of Fracture Network in Shale Gas Formation," $1^{\text {st }}$ International Discrete Fracture Network Engineering Conference, Vancouver, Canada, October 19-22, 2014.

Huang, H., B. Spencer, and J. Hales, "Discrete Element Method for Simulation of Early-Life Thermal Fracturing Behavior in Ceramic Nuclear Fuel Pellets," Nuclear Engineering and Design, Vol. 278, 515-528, October 15, 2014. 
Huang, H., E. Mattson, and R. Podgorney, "Physics-Based Modeling of Hydraulic Fracture Propagation and Permeability Evolution of Fracture Network in Shale Gas Formation," 48th U.S. Rock Mechanics / Geomechanics Symposium (ARMA 2014), Minneapolis, MN, June 1-4, 2014.

Meakin, P., H. Huang, A. Malthe-Sorenssen, and K. Thogersen, "Shale Gas: Opportunities and Challenges," Environmental Geosciences, Vol. 20, 151-164, 2013.

\section{Presentations}

Huang, H., "Physics-Based Modeling of Hydraulic Fracture Propagations and Permeability Evolutions During and After Stimulations," AAPG Geosciences Technology Workshop: Geomechanics and Reservoir Characterization of Shales and Carbonates, Baltimore, MD, July 16-17, 2013.

Huang, H., "Simulations of In Situ Production Processes in Reservoir Modeling," University of Utah Unconventional Fuels Conference, Salt Lake City, UT, May 2013.

Huang, H., "Multiphysics Modeling and Simulations of Coupled Thermal-Hydro-Mechanical-Chemical Processes in the Subsurface," University of Utah Unconventional Fuels Conference, Salt Lake City, UT, May 2013.

Huang, H., M. Plummer, and R. Podgorney, "Simulated Evolution of Fractures and Fracture Networks Subject to Thermal Cooling: A Coupled Discrete Element and Heat Conduction Model," in Proceedings of 38th Workshop on Geothermal Reservoir Engineering, Stanford University, Stanford, CA, February 11-13, 2013. 


\title{
13-027 - Diagnostics of Advanced Energy Storage Materials
}

\author{
Eric J. Dufek, Michael V. Glazoff, Lucia M. Petkovic, and Doug Akers
}

The emergence of new materials for electrochemical energy storage necessitates the development of means to effectively evaluate how different materials interact with each other and how processing techniques impact overall performance. This project is focused on the implementation of multiple optical, spectroscopic, and electrochemical techniques to more fully understand how performance of battery electrodes and systems is impacted as a function

a

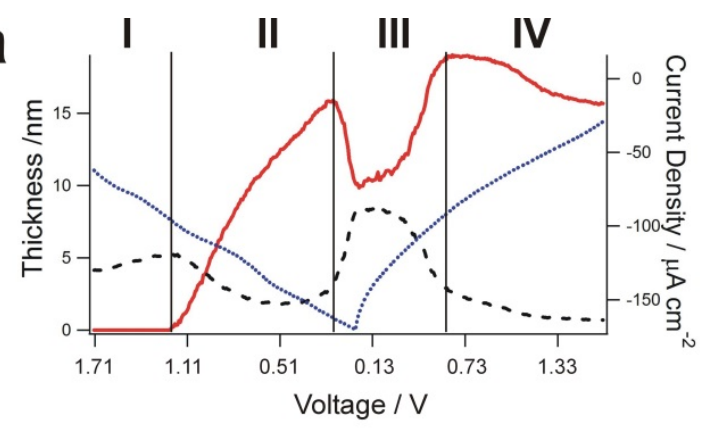

b

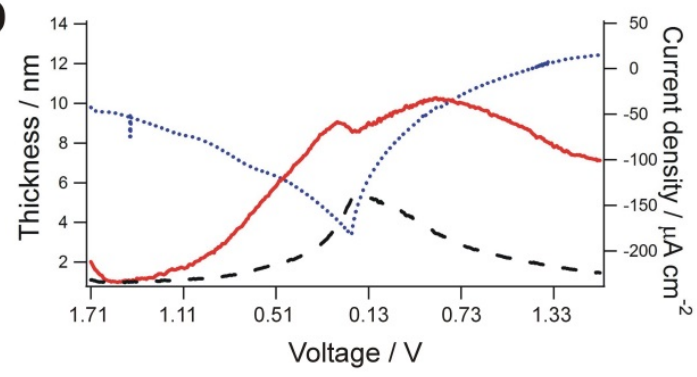

C

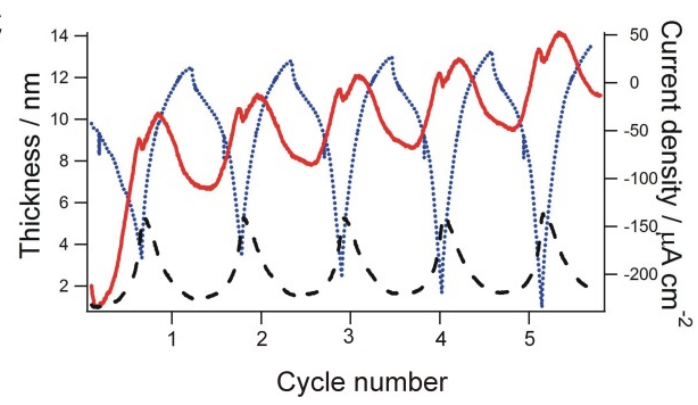

Figure 1. Spectroscopic ellipsometry and electrochemical data of an electrode cycled in 1:1 EC:EMC (a) or 1:4 EC:EMC $(b, c)$. The red lines correspond to the thickness of the inner layer, dashed black with the outer layer thickness of the SEl. Current density is denoted in blue. of processing and chemistry. Of particular interest to the project is the use of spectroscopic ellipsometry, Fourier transform infrared spectroscopy, imaging techniques such as scanning electron microscopy, other physical characterization methods, and electrochemical performance. The prime materials and interactions of interest to the project are those that occur within the electrolyte, the binder, and with the active materials in the electrodes.

Lithium (Li)-ion battery performance is impacted by a multitude of factors, including active materials, electrode configuration, composition, electrode thickness, porosity, and environmental variables. Formulated composite electrodes for Li-ion batteries are typically composed of an active material, a binder, and a conductive additive such as carbon. During processing, cast electrodes can be calendared to different thicknesses and porosities to influence performance. During cycling, changes in materials, such as the formation of the solid electrolyte interphase (SEI) and in-expansion induced fracturing of materials, can significantly impact long-term battery performance and can have impacts on secondary degradation mechanisms. It is difficult to fully grasp degradation using a single method, thus the implementation of multiple methods is necessary to obtain an in depth analysis. Key objectives of using complimentary methods include the ability to follow SEI change during cycling, identifying chemical changes which occur with variable binder compositions, following how mechanical stress and electrode processing impact electrode performance, computationally identifying morphological aspects of electrodes, and modeling how electrode structure can change during cycling. A secondary purpose of the project is to develop methods that show broad applicability to cathode and anode systems as well as relevancy to emerging battery chemistries. 


\section{ENERGY \& ENVIRONMENT SCIENCE \& TECHNOLOGY}

\section{Summary}

Technical progress to date has been made in following the growth of the SEI using a multi-component spectroscopic ellipsometry model that more closely aligns with the physical description of the thin film. A brief summary of the work is shown in Figure 1 where the thickness of the inner and outer components of the SEI can be seen to vary as a function of both voltage and overall number of cycles. Additional changes have been identified in the thickness of the SEI as the electrolyte composition changes. The result of these investigations was the publication of a peer-reviewed paper in ECS Electrochemistry Letters.

Other activities in the project have looked at the performance degradation of silicon (Si)-based anodes. The work has involved the role binders play in performance degradation, the impact of the processing technique, and thirdly, how morphological attributes of electrodes can be identified using advanced computational techniques and subsequently altered using models to simulate material changes over cycling. With regard to the role of binders, it has been found that neutralizing polyacrylic acid with a suite of different alkali metal hydroxides can lead to shifts in the chemical structure of the binder as evidenced by shifts in the Fourier transform infrared spectroscopy spectrum of both the native binder material and in as-formulated electrodes. A typical image of an electrode surface is shown in Figure 2. Altering the cation was found to have direct impacts on overall cycling performance where potassium (K)-based polyacrylates had less capacity loss than Li polyacrylates using the same Si-based anode. In addition to following the role of binder, it has been observed that performance both in terms of degradation rate and total electrode capacity can be significantly altered by modifying the thickness of the electrode.

Using many of the images acquired during the investigations of binder impact and active material studies, it has been possible to extract information on the morphological features of the composite electrodes and

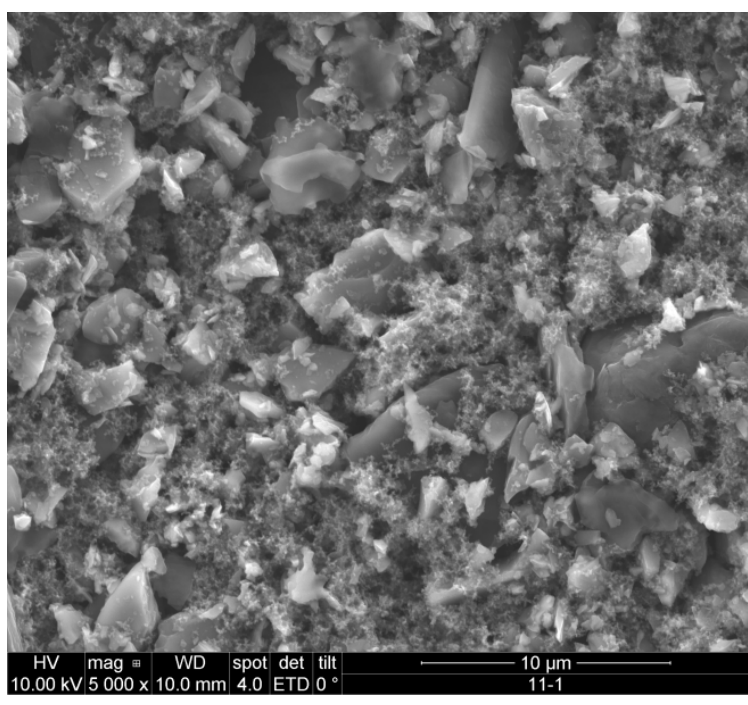

Figure 2. Scanning electron microscopy image of a Si-based composite electrode with a $\mathrm{K}$ polyacrylate binder. then add predictive aging algorithms to predict how the structure will change as a function of cell cycling. To date, this has been applied to the growth of a thin film on as-formed electrodes, which mimics the initial growth of the SEl.

\section{Benefits to DOE}

The ability to follow changes in the interfacial regions provides a direct route to identify needs when optimizing performance. By identifying these needs, routes for improved capacity, long-term battery performance, and safety can be made. All are significant in meeting DOE's goal of increasing the electrification of the vehicle fleet in the United States. By improving performance, advanced characterization methods increase the time a battery can be used and decrease the size of the needed battery. This is beneficial in minimizing the environmental impact of battery manufacturing. As a whole, the techniques employed in this work will advance knowledge of how interfaces change in complex systems and will provide important information for Li-ion and other new emerging battery chemistries. 


\section{Publications}

Dufek, E., "Evaluation of the SEI Using a Multilayer Spectroscopic Ellipsometry Model," ECS Electrochemistry Letters, Vol. 3, A108-A111, 2014.

\section{Presentations}

Dufek, E., L. Petkovic, H. Rollins, and J. Klaehn, "Use of Complimentary Methods to Evaluate Energy Storage Interfaces," 248th National Meeting of the American Chemical Society, San Francisco, CA, August 2014.

Dufek, E., and L. Petkovic, "Correlating Electrochemical and Spectroscopic Ellipsometry Signals to Evaluate Energy Storage Interfaces," 224th Meeting of the Electrochemical Society, San Francisco, CA, October 2013.

Dufek, E., M. Glazoff, and K. Gering, "Morphological Analysis for the Evaluation of Electrode Structures," 224th Meeting of the Electrochemical Society, San Francisco, CA, October 2013. 


\title{
13-065 - Multi-Domain Modeling, Simulation, and Integration Tools - Hybrid Energy Systems
}

\author{
Humberto E. Garcia, Wenbo Du, Christiaan Paredis, ${ }^{1}$ William R. Binder, ${ }^{1}$ and Rob Hovsapian
}

The principal objective of this project is to develop (from formulation to validation) dynamic modeling, integration, simulation, controls, and optimization capabilities for studying various aspects of hybrid energy systems (HES). The purpose of this project is to obtain the necessary tools and experience to enable the design, analysis, operation, and optimization of HES using high-fidelity dynamic models and advanced simulation and optimization techniques. Specifically, the following capabilities have been identified as being crucial to meeting the objectives of the project: dynamic modeling and simulation (M\&S) tools (including creation of a modeling library to take advantage of model reusability), integration tools for synergizing the various tools and experiences available, optimization capabilities for both offline (design optimization) and online (operations) applications, and controls strategies, architectures, and algorithms to address challenges such as variability and uncertainty introduced by renewable sources and time-varying demands.

\section{Summary}

Progress towards meeting the modeling, integration, simulation, controls, and optimization capabilities needed for the objectives of the project include the creation and organization of models and packages within a comprehensive Modelica library for HES research, development, and demonstration. The HES library takes advantage of the reusability of models to enable models of novel HES configurations to be quickly assembled and studied. Our M\&S efforts have already utilized this library to create several modeling configurations of HES solutions for different purposes such as parametric analysis, optimization, integration, and control.

Additionally, two separate methodologies for creating dynamic reduced order models have been formulated and implemented based on surrogate modeling (to create purely numerical approximations of physical models) and physics-enhanced "hybrid" modeling, which uses simplifying assumptions to combine numerical approximations with physics-based equations. Reduced order models of several HES components have been developed to greatly improve the speed of simulations and enable the optimization of several design variables.

In the domain of model integration, two platforms-the Functional Mock-up Interface and the Building Controls Virtual Test Bed-have been successfully adopted for model exchange and co-simulation. Functional Mock-up Interface has been used to obtain optimization results that were presented in papers at the 2014 Modelica and International Conference on Renewable Energy Research and Applications conferences, and the Building Controls Virtual Test Bed has been successfully used to integrate Modelica thermo-mechanical and Simulink electrical models for co-simulation.

\footnotetext{
${ }^{1}$ Georgia Institute of Technology
} 
Preliminary results for evaluating an evolvable HES configuration using a real options formulation have shown the relative impact of several sizing variables on long-term profit and net present value metrics. In brief, four key areas have been addressed:

1. Technical challenge: variability in energy production and consumption; technological solution: dynamic M\&S tools.

a. Structure-independent: to solve problems of any structure, hence facilitating model reusability

b. Multi-domain: effective integration of computational and physical systems (including hardware-in-the-loop) from diverse disciplines

c. Open: allow modification of component modules to accommodate specific needs

d. Hybrid: time- and event-driven modeling

e. Non-proprietary: ease collaboration via open licensing.

2. Technical challenge: variability $M \& S$ and hardware-in-the-loop environments; technological solution: systems integration tools.

a. Model exchange (e.g., Functional Mock-up Interface)

b. Co-simulation (e.g., Ptolemy II/Building Controls Virtual Test Bed, High-Level Architecture, Parallel Virtual Machine, TLK Inter Software Connector, Dynamic Link Library)

c. Near real time/real time computational integration (e.g., desktops, real-time digital simulator)

d. Hardware-in-the-loop

e. Verification and validation.

3. Technical challenge: variability in component and configuration options and energy markets; technological solution: systems design and operations optimization tools.
a. Classical design optimization
b. Real options design optimization
c. Real-time operations optimization
d. Dynamic reduced order models.

4. Technical challenge: variability, uncertainty, and uncontrollability in energy production and consumption; technological solution: monitoring and controls tools.

a. Local and global controls: components/systems

b. Anticipatory/real-time optimization, resilient controls: to deal with uncertainties

c. Hybrid: to accommodate time- and event-driven dynamics.

\section{Benefits to DOE}

HES have been identified as a promising architecture for resolving the difficulties of integrating clean energy technologies into grid infrastructures and as a key element towards grid modernization. If successful, HES can be a critical component of the nation's future energy portfolio, allowing usage of clean energy technologies to expand and therefore reduce carbon $(\mathrm{C})$ emissions and other harmful effects. Challenges include high variability and 
uncertainty in renewable resources and demands, grid instability arising from high levels of renewable penetration, and uncertainty in historical data combined with the high capital cost of nuclear plants. The modeling, integration, simulation, controls, and optimization capabilities developed in this project enable the types of analysis necessary to reduce risks derived from uncertainty and variability.

\section{Publications}

Binder, W., W. Du, C. Paredis, and H. Garcia, "Design Optimization of a Hybrid Energy System using Real Options," in preparation, 2014.

Du, W., H. Garcia, W. Binder, and C. Paredis, "Value-Driven Design and Sensitivity Analysis of Hybrid Energy Systems using Surrogate Modeling," International Conference on Renewable Energy Research and Applications (ICRERA) 2014, Milwaukee, WI, October 19-22, 2014.

Du, W., H. Garcia, and C. Paredis, "An Optimization Framework for Dynamic Hybrid Energy Systems," Proceedings of the 10th International Modelica Conference, Lund, Sweden, 767-776, March 10-12, 2014.

Binder, W., C. Paredis, and H. Garcia, "Hybrid Energy System Modeling in Modelica," Proceedings of the 10th International Modelica Conference, Lund, Sweden, 979-988, March 10-12, 2014.

\section{Presentations}

Du, W., H. Garcia, W. Binder, and C. Paredis, "Value-Driven Design and Sensitivity Analysis of Hybrid Energy Systems using Surrogate Modeling," International Conference on Renewable Energy Research and Applications (ICRERA) 2014, Milwaukee, WI, October 19-22, 2014.

Garcia, H., et al., “Nuclear Hybrid Energy Systems: Challenges \& Opportunities,” May 2014.

Binder, W., C. Paredis, and H. Garcia, "Hybrid Energy System Modeling in Modelica," Presentation at the 10th International Modelica Conference, Lund, Sweden, March 10-12, 2014.

Du, W., H. Garcia, and C. Paredis, "An Optimization Framework for Dynamic Hybrid Energy Systems," 10th International Modelica Conference, Lund, Sweden, March 10-12, 2014.

Garcia, H., "Energy Integration \& Optimization Using Dynamic Systems Models \& Hardware-in-the-Loop Validation," NUC Meeting, January 29, 2014.

Garcia, H., et al., "Process Integration \& Optimization Using Dynamic Systems Models," AIChE CAST Webinar Series, Idaho Falls, ID, January 14, 2014.

Garcia, H., "Integration of Small Modular Reactors within Clean \& Sustainable Hybrid Energy Systems," Small Modular Reactors Conference, Idaho Falls, ID, October 30, 2013. 


\title{
13-079 - Diverse Biological Factories for Sustainable Manufacturing
}

\author{
Dayna L. Daubaras
}

This project is an effort to support sustainable manufacturing, a key area of innovative manufacturing within the DOE-Energy Efficiency and Renewable Energy Advanced Manufacturing Office. We are creating unique biological factories to facilitate manufacturing processes for the energy efficient production of commodity chemicals, fine chemicals, fuels, and biological products. Biological systems can be engineered to express synthetic catalytic capabilities and allow the conversion of central metabolic intermediates to produce chemical products. However, only a few host systems are available for genetic optimization and large scale production under a limited set of conditions. These commonly used host systems require expensive carbon sources for growth and extensive engineering to modify the central metabolic pathways. The focus of this project is to identify and evaluate host organisms with inherent metabolic pathways requiring minimal engineering and less expensive carbon sources to produce chemicals economically. The selected hosts should function under extreme conditions $(\mathrm{pH}$, temperature, pressure) to facilitate industrial processes. The chemicals we propose to target are those currently sourced from petroleum resources and energy intensive chemical processes.

\section{Summary}

In the first year of this project, we selected a halophilic Archaeal microorganism for the production of isoprenoid compounds using the host mevalonate metabolic pathway to provide the appropriate intermediates. We genetically engineered this organism using synthetic DNA coding for host optimized plant terpene synthases. Specifically, we had two plant isoprene synthase genes synthesized and introduced them on extra-chromosomal elements into the organism. In addition, we deleted a gene in the metabolic pathway for carotenoid production and replaced it with the synthetic isoprene synthases on the host chromosome. The targeted deletion of a carotenoid biosynthetic gene using a cassette based system served a two-fold purpose for this project: (1) to divert central metabolic intermediates away from carotenoid production and toward the production of alternate isoprenoid compounds and (2) to generate a colorimetric screening tool for introducing synthetic genes on the host chromosome. The compound carotenoid is responsible for the orange color found in the cell wall of the halophilic organisms. When the biosynthetic gene encoding phytoene synthase, crtB, was deleted and replaced with a synthetic isoprene synthase, the strain had a white phenotype. The goal now was to express the isoprene synthase gene under control of the phytoene synthase regulation, as well as from an extra-chromosomal plasmid under multiple regulatory elements. Unfortunately, after testing three different regulatory control elements, we were not able to detect isoprene production using two different synthetic isoprene synthase genes. We also could not detect the tagged isoprene synthase protein using metal affinity purification from the various expression strains, leading us to believe the synthetic genes were not properly processed in this strain. 


\section{ENERGY \& ENVIRONMENT SCIENCE \& TECHNOLOGY}

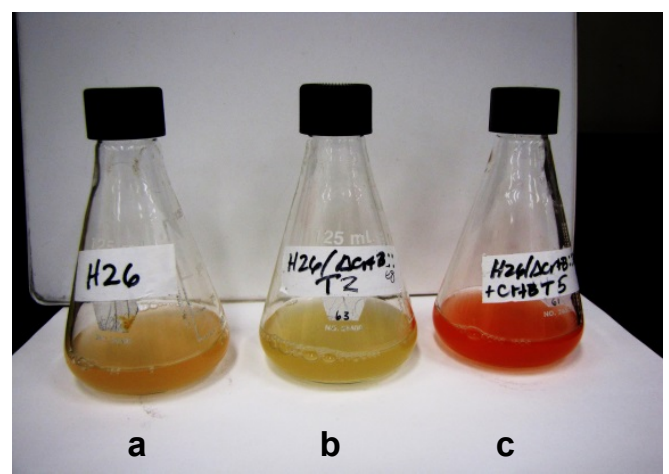

Figure 1. Flasks with the various strain types. (a) The host strain with wild type carotenoid production. (b) The carotenoid crtB gene deletion strain. (c) The over-expressed carotenoid crtB strain.
To test the efficacy of the genetic system we used during the first year, we complemented the carotenoid chromosomal gene deletion by copying the host crtB gene by polymerase chain reaction and placing it on the various extra-chromosomal plasmids replacing the synthetic isoprene synthase gene. The expression of this polymerase-chain-reaction-amplified crtB gene allowed the colored carotenoid compound production to be restored to the white deletion strain. In fact, we increased the carotenoid levels, as evidenced by the dark red colored cells (Figure 1.). This result confirms the colorimetric based cassette system we generated has the capacity to overproduce proteins involved in isoprenoid production. Currently, we are quantifying the levels of carotenoid production in this strain.

In summary, we successfully created and tested a colorimetric system for identification of genetic modifications to the host strain,

an important step required for rapid screening. The interchangeable genetic modifications we created are versatile enough to easily replace the current synthetic catalyst, isoprene synthase, with other valuable catalysts. In the third year, we will evaluate the use of alternate synthetic terpene synthase encoding genes, specifically for the production of pinene.

\section{Benefits to DOE}

Creating production strains using diverse organisms more easily amenable to industrial scale up processes will improve the efficient use of energy and decrease the nation's dependence on foreign oil. Many biological processes are considered more environmentally friendly due to the decreased use of energy in scale up processes, the reduction in $\mathrm{CO}_{2}$ emissions compared to chemical processes, and the value added back to a primary process by using otherwise "waste" products as biological substrates for growth. Reducing our dependence on foreign oil by generating hydrocarbon compounds through biological processes is invaluable to national energy security.

\section{Publications}

A manuscript is in preparation. The FY 2014 research did not yield the anticipated results, so the focus of the intended manuscript is being revised for FY 2015 submission. 


\title{
13-114 - Multiphase Fluid Trapping in Propped Hydraulic Fractures
}

\author{
Earl D. Mattson, Mitchell A. Plummer, and Ghanashyam Neupane
}

Hydraulic fracturing of shale reservoirs for natural gas production has created public concern about the amount of water used in the process and about potential contamination of drinking waters from the fracturing process and the handling and disposal of returned fluids. At present, there does not appear to be any clear agreement within the industry as to whether the most important damage that hinders gas removal is via alteration of fracture proppant conductivity or via alteration of the formation directly adjacent to the fracture. This is in part due to the difficulty of identifying unique characteristics in either the flowback water or the gas rate decline in these two instances. While this proposal focuses primarily on water in the proppant pack, we will also consider potential impacts to the matrix interface in our numerical simulations and supporting experiments by examining proppant embedment, proppant rearrangement, and proppant digenesis under field conditions for unconventional fossil energy extraction as well as potential use in enhanced geothermal systems.

\section{Summary}

The general approach we have taken is to refine our initial conceptual model for fluid entrapment in propped fractures in shale-gas reservoirs. We have implemented this conceptual model in a numerical code and compared simulation results to previously conducted laboratory experiments by our industrial collaborator that provided key information about the distribution of water saturation in propped fractures. Simulation validation experiments are being conducted at the INL 2-meter centrifuge facility. A 2-D geocentrifuge experiment has been built to conduct scaled flow and transport experiments of gas movement in a water-filled hydraulic fracture. The centrifuge offers the advantage of scaling the fracture height to simulate more realistic field conditions. The simulations and experimental results indicate that the water and gas flow pathways do not coincide, suggesting that different portions of the fracture are important at different times during the drainage.

As part of this LDRD's project outreach to industry, INL has been accepted as a guest member in Stim-Labs industrial proppant consortium. We attended three consortium meetings and presented initial results to the members. A paper was submitted to the Society of Professional Engineers with our industrial collaborators (Stim-Labs, Inc.) on evaluating the flow field of water in the standard Cooke testing cell using discrete element method modeling. In response to this paper, Earl Mattson was invited to participate in the American Petroleum Institute subcommittee to review the proposed changes to proppant testing procedures.

Due in part to activities conducted under this LDRD, a related proposal was submitted to the Research Partnership for Secure Energy for America on fracturing shale reservoirs using a gas instead of water (Mattson, E., and A. Ghassemi, "Evaluation of $\mathrm{CO}_{2}$ as a Fracturing Fluid for Shale Reservoirs," RFP2012UN001, INL/PRO-13-29304).

A University of Idaho mechanical engineer summer intern, Nathan Petersen, was funded under this LDRD and was awarded one of the University of Idaho's CAES Energy Scholarships from CAES for Idaho students interested in performing energy-related research. Nathan also presented his LDRD work at the annual INL intern poster symposium. His poster and presentation were awarded as runner-ups in two of the categories: best technical content and best oral presentation. 
Activities to assess engineering solutions for gas/water interaction in a proppant-filled hydraulic fracture were addressed in FY 2014. These activities included an outreach to the University of Utah's Energy and Geoscience Institute professor Dr. Joesph Moore and his Ph.D. student Clay Jones. To support this outreach, INL hired Dr. Ghanashyam Neupane to perform batch chemical reaction studies to examine the digenesis of proppants under high temperature geothermal conditions. These experimental results are expected to be presented at the Stanford Geothermal Workshop in January 2015.

In addition, we examined the interaction of unconventional fossil resources and proppants for two rock types. For the first rock type, oil shale microstructure layering and its effects on proppant embedment was studied using the CAES nano-indentor. Results of this work were presented at the 34th Oil Shale Symposium and suggest that our initial conceptual model of treating the oil shale as a homogeneous anisotropic material was correct. Proppant embedment into shale gas reservoir rocks was also evaluated. This work was published at the Discrete Fracture Network Engineering Conference with Shell oil as co-authors and suggests that the shale rock can exhibit plastic-like geomechanical responses at high temperatures which would account for larger proppant embedment in deep shale gas reservoirs.

\section{Benefits to DOE}

Efforts funded under this LDRD project are in line with DOE national security missions through providing a better understanding and possible solutions to increasing natural gas recovery from tight reservoirs and renewable energy production using geothermal fluids. DOE has supported this type of activity through its National Energy Technology Laboratory and Research Partnership for Secure Energy for America solicitations for proposals in the past years. Research conducted under this proposal will also benefit the Environmental Protection Agency Hydraulic Fracturing Study; the DOE Subsurface Technology and Engineering Research, Development, and Demonstration cross cut efforts; and the DOE Geothermal Technology Office's Frontier Observatory for Research in Geothermal Energy effort to demonstrate enhanced geothermal system in the field.

\section{Publications}

Frash, L., M. Gutierrez, A. Tutuncu, J. Hampton, J. Hood, H. Huang, and E. Mattson, "Laboratory-Scale Study of Grain Structure's Influence on Hydraulic Fracture 3D Geometry," INL/JOU-14-34004, International Journal of Rock Mechanics and Mining Sciences, submitted.

Frash, L., M. Gutierrez, A. Tutuncu, J. Hampton, J. Hood, H. Huang, and E. Mattson, "Critical State Hydraulic Fracture Geometry in Unconfined Acrylic and Granite," SPE Journal, submitted.

Mattson, E., H. Huang., X. Li., and H. Lopez, "2D Discrete Element Modeling and Experimental Results of Proppant Embedment into Fracture Rock Walls," INL/CON-14-32142, International Discrete Fracture Network Engineering Conference, Vancouver, Canada, October 19-22, 2014.

Frash, L., J. Hood, M. Gutierrez, E. Mattson, and H. Huang, "Laboratory Measurement of Critical State Hydraulic Fracture Geometry," 48th U.S. Rock Mechanics/Geomechanics Symposium, Minneapolis, MN, June 1-4, 2014.

Huang, H., and E. Mattson, "Physics-Based Modeling of Hydraulic Fracture Propagation and Permeability Evolution of Fracture Network in Shale Gas Formations," INL/CON-14-32186, 48th U.S. Rock Mechanics/Geomechanics Symposium, Minneapolis, MN, June 1-4, 2014. 
Mattson, E., H. Huang, and M. Conway, "Discrete Element Modeling Results of Proppant Rearrangement in the Cooke Conductivity Cell," INL/CON-13-29950, SPE (Society of Petroleum Engineering), Hydraulic Fracturing Symposium, February 2014.

\section{Presentations}

Neupane, G., E. Mattson, and H. Huang, "Preliminary Investigation of the Oil Shale Geomechanical Micro Structure Distribution Using Nanoindentation Technique," INL/CON-14-33385, 34th Oil Shale Symposium, Colorado School of Mines, Golden, CO, October 2014.

Mattson, E., and M. Plummer, "Proppant Fluid Drainage Studies Using a Geocentrifuge," INL/CON-13-29579, Stim Labs Proppant Consortium Meeting, Rhodent, CA, July 19, 2013. 


\title{
14-078 - Extended Stability Gamma-Gamma Prime Containing Nickel-Base Alloys
}

\author{
Laura Carroll, Mark Carroll, Tresa Pollock, and Subhashish Meher
}

Dispatchable solar power generation is one of the key building blocks for the 21 st century grid, while the nuclear and fossil industries continue to demand improved efficiencies to remain competitive. In each case, increasing system efficiency requires structural materials that have properties that exceed those of the present state-of-the-art. Fortunately, the material requirements of the specific hot section components from three industries are similar. All require an extended stability alloy capable of operating at $1000^{\circ} \mathrm{C}$ for up to 100,000 hours either to extract the maximum amount of efficiency from the system or to allow the system to operate at temperatures that render the configuration technologically feasible. The objective of the project is to develop a $\gamma-\gamma$ ' nickel (Ni)-base alloying and microstructural strategy capable of meeting these requirements that is substantially more stable with regard to secondary phase evolution, resulting in a consistent set of mechanical properties at elevated temperatures while demonstrating superior oxidation resistance. The benefit in creep strength of introducing a $\gamma^{\prime}$-containing superalloy with long term stability is expected to be an increase of greater than $200^{\circ} \mathrm{C}$. The alloying strategy being carried out deviates from the present state-of-the-art in $\gamma-\gamma$ ' superalloys in that it is engineered specifically to be suitable for extended service durations in extreme environments.

Performance in these specialized conditions is necessary not only for specific components in solar thermal, receiver-based turbine systems but also in providing an unmatched structural material for an intermediate heat exchanger for use in nuclear and fossil applications.

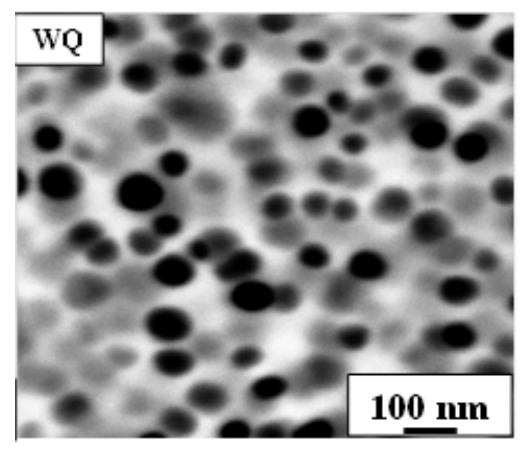

Figure 1. Preliminary $\gamma-\gamma^{\prime}$ microstructure of a Ni alloy after initial processing.

\section{Summary}

Casting and analysis of two iterations of systematically designed experimental $\mathrm{Ni}$ and cobalt ( $\mathrm{Co}$ ) based alloys, with compositions uniquely selected to achieve stable microstructures at very high temperatures after tailored processing steps, have been achieved. The goal of the preliminary castings was to create either a single phase $\gamma$ alloy or a homogeneous distribution of very fine precipitates following specialized solution heat treatments. Initially, a microstructural investigation was conducted in order to evaluate the ability to retain a solid solution of the $\gamma$ matrix after an appropriate solution heat treatment. Nucleation (likely homogeneous) of the $\gamma$ ' precipitates in the first round of alloys, despite rapid quenching, suggested focusing the second series of alloy compositions on those with a greater separation between the solutioning temperature and the $\gamma$ ' solvus temperature. The gap between the two temperatures for potential second

round alloys was much larger than the first iteration as determined by advanced predictions using the ThermoCalc $®$ thermodynamic database.

The second series of castings included multiple alloys of three unique types, each aimed at a high level of $\gamma$ ' stability. Figure 1 illustrates that although the $\gamma$ ' phase precipitated during cooling from solution temperatures, a suitable microstructure for further stability and coarsening studies along with an analysis of processing effects have resulted. The specific $\gamma$ ' coarsening behavior and precipitate-coarsening relationships are currently being developed through a combination of experimental and modeling techniques. Laboratory-based, long-time, 


\section{ENERGY \& ENVIRONMENT SCIENCE \& TECHNOLOGY}

high-temperature exposures and subsequent microstructural characterization reveals the inherent coarsening behavior, as shown for a selected alloy in Figure 2. Extensive atom probe tomography and directed theoretical calculations are in progress to assess the compositional and interfacial characteristics necessary to model the long term kinetic microstructural behavior utilizing TC PRISMA®.

Based on the high-volume fractions of $\gamma^{\prime}$, these experimental alloys are extremely creep resistant relative to current state-of-the-art alloys (Alloy 617, Alloy 230) considered for service for extended durations at high temperatures. To quantify these characteristics, components of the creep resistance of idealized microstructures have been modeled to guide the selection of experimental alloy compositions. These mechanistic models link alloy microstructure to creep resistance and enable the prediction of an optimum combination of precipitate size, volume fraction, and interfacial energy that will maximize the resistance to $\gamma$ ' shearing/bypass. Characteristics of actual microstructures observed experimentally (Figure 3) provide the basis

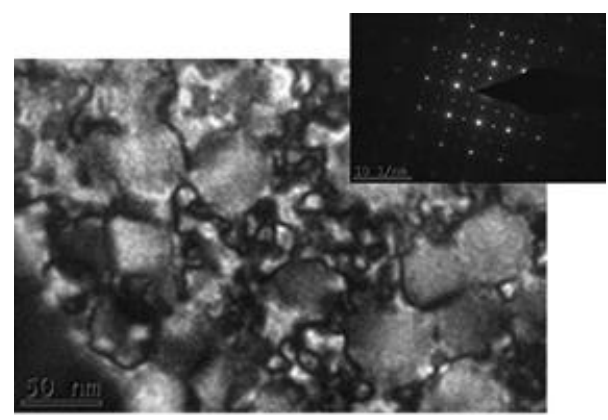

Figure 3. Dark field transmission electron microscope image used to characterize the precipitate size and volume fraction of the multiple generation $\gamma^{\prime}$ precipitates in a r microstructure of a Co alloy.

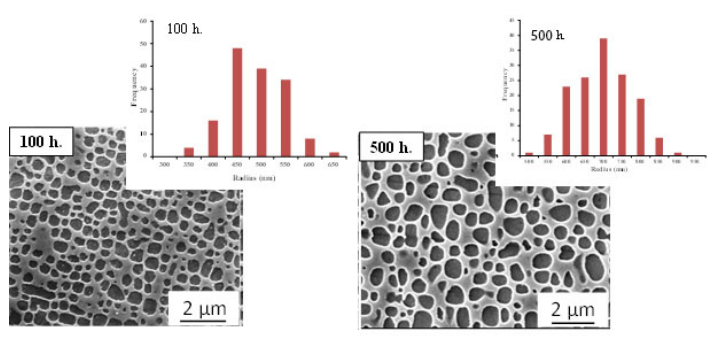

(a)

(b)

Figure 2. A Ni alloy with a relatively stable $\gamma-\gamma^{\prime}$ microstructure as evidenced from coarsening studies at $1000^{\circ} \mathrm{C}$ and $100 \mathrm{hr}$ (a) and $500 \mathrm{hr}(\mathrm{b})$. for quantification of the creep strength of experimental alloys. These models will direct the ongoing microstructural development, selection of processing conditions, and alloy compositions. The integrated use of advanced computational methods (ThermoCalc $®$, microstructure/strength mechanistic-based models, and design of experiment methods) and experimental methods (transmission electron microscopy, atom probe tomography, and scanning electron microscopy) are enabling targeted alloy development strategies through linking of the alloy composition (microstructure) properties to develop materials solutions that enable the long-term deployment of very high temperature components.

\section{Benefits to DOE}

This project is developing an alloying/microstructural strategy for $\gamma-\gamma^{\prime}$ superalloys with inherent long-term stability by exhibiting tailored,

unique microstructures that make them ideal for extended service durations at high temperatures necessary for specific solar/fossil/nuclear energy components that will enable increased efficiencies and energy production. For example, a stable $\gamma-\gamma$ ' alloy would enable a solar thermal receiver material capable of withstanding a compressed air Brayton-cycle in concentrating solar power technologies. Currently, the state-of-the-art materials available for very high temperature structural applications are limited in either strength or duration of service due to an evolving $\gamma^{\prime}$ microstructure.

\section{Presentations}

Meher, S., L. Carroll, M. Carroll, and T. Pollock, "Microstructural Stability in High Refractory Containing Nickel-Base Superalloys," TMS Annual Meeting, Orlando, FL, February 2015. 


\section{4-079 - Second Generation Switchable Polarity Solvent Draw Solutes for Forward Osmosis}

Aaron D. Wilson, Christopher J. Orme, Josh McNally, Frederick F. Stewart, and Jeffrey R. McCutcheon ${ }^{1}$

The Switchable Polarity Solvent (SPS) Forward Osmosis (FO) process can purify water from extremely concentrated feeds containing components such as salts, organics, inorganics, and biologics. INL has demonstrated that a dimethylcyclohexylamine SPS draw solution can provide osmotic flux against a $226,000 \mathrm{ppm}$ tds $\mathrm{NaCl}$ solution. SPS FO functions at a fraction $(<0.5)$ of the cost of existing methods through a thermolytic cycle that avoids the distillation of water but still uses heat energy. The drawback of this initial demonstration of SPS FO is the aggressive solvent characteristics of dimethylcyclohexylamine, which damages membranes and a wide range of rubbers and plastics. Advancement of SPS FO to industrial implementation requires an SPS that retains the high osmotic pressures of dimethylcyclohexylamine without the material incompatibilities. In this project, materials will be developed as draw solutes for FO systems that have high osmotic pressures, low viscosities, and reasonable costs. These materials will require properties similar to previously studied materials while avoiding material degradation, reduced toxicity, reduced membrane permeation, and improved SPS switching behavior. This work will include the synthesizing and characterization of amines. With a viable SPS, an integrated demonstration of an SPS FO process will be developed based on the most effective SPS candidate.

\section{Summary}

The first effort in FY 2014 was to complete and publish a structure-function model of the SPS's capability to form concentrated solutions. This structure function model allowed us to identify our Gen II SPS with a higher molecular weight than the SPS used to demonstrate the SPS FO process. The higher molecular weight provides a number of advantages, including: (a) reduced vapor pressure/odor; (b) better kinetics, lower temperatures, and more complete polar to non-polar phase transitions (degassing); (c) lower water solubility in the non-polar form; (d) better materials compatibility; (e) and lower likelihood of passing through membranes for FO/RO applications. The Gen II SPS adds all these advantages while retaining the high osmotic pressure and low production cost of diemethylcyclohexylamines. Battelle Energy Alliance, LLC, has submitted a patent application (BA-808) for the use of Gen II SPS as a water treatment.

We next conducted a concentration dependent study of Gen II and two control SPSs. This work has resulted in a submitted paper addressing mass transport properties and solution-state speciation, generalized to all SPSs. These concentration dependent properties are fundamental to process design and optimization of any application of SPS.

We have also generated ( \pm -trans- $N, N, N^{\prime}, N^{\prime}$-tertamethyl-1,2-Diaminocyclohexane at the $300 \mathrm{~g}$ scale and demonstrated that they are effective SPS materials. The diamine can reach $80 \mathrm{wt} \%$ in its polar form before forming a crystalline material matching the performance of the best monoamines. In the next year, concentration performance metrics will be obtained for the diamine and compared to our Gen II SPS.

Our collaboration with Prof. McCutcheon is yielding water flux data, reverse solute flux, membrane stability, and solute/membrane parameters for several membranes. This collaboration is expected to result in the submission of two papers in the next year.

\footnotetext{
${ }^{1}$ University of Connecticut
} 
A postdoctoral researcher, Josh McNally joined the project mid-year and is conducting a Discrete Fourier Transform analysis to further the structure-function model. To supply the Discrete Fourier Transform studies with an effective starting point, we obtained an $\mathrm{x}$-ray crystal structure of a dimethylcyclohexylammonium bicarbonate. This structure featured a hydrogen $(\mathrm{H})$ bonded bicarbonate dimer. Recently, Frank Weinhold at the University of Wisconsin and Roger Klein proposed the existence of anti-electrostatic $\mathrm{H}$ bond complexes which would conclusively demonstrate a dominant covalent component of the $\mathrm{H}$ bond. Our bicarbonate dimer is an example of an anti-electrostatic $\mathrm{H}$ bond and collaboration has been initiated to further develop the anti-electrostatic $\mathrm{H}$ bond work and obtain a neutron structure of our bicarbonate dimer via the Oak Ridge National Laboratory Spallation Neutron Source User Facility. The results should influence our understanding of the $\mathrm{H}$ bond, which will have a wide impact on our understanding of chemistry from the classroom to the research laboratory.

Based on our published work (including the paper from this LDRD), we were contacted by a start-up company, Rebound Technology working in the field of modest to large-scale refrigeration. The Rebound interaction has developed into a collaboration that has already resulted in an application for support to Advanced Research Projects Agency-Energy's "open call." This collaboration is built around a new field of use for SPS that would not have been possible without the fundamental work developed in this LDRD.

\section{Benefits to DOE}

Water treatment and desalination is an energy intensive process with room for improvement. Reducing this energy cost would meet DOE's mission, but the connection between water and energy runs deeper. The current energy infrastructure requires water to produce energy (evaporative cooling, biomass and algae production) and obtain energy resources (well injection [fracking] water). Water used by the energy industries eventually requires treatment (oil and gas water, blowdown water, and many others). The timeliness of this work is well illustrated by the issues at Turkey Point Nuclear Generating Station and the nationwide draught conditions, especially those in California's central valley. Mission impact beyond DOE would be best described in terms of energy, water, and food security. The technology under development may also have application for water production at forward operating bases. This project is expected to develop scalable water treatment technology that can reduce the cost of producing and processing waters relevant to the energy industry and military.

SPSs are a rapidly developing field of research. Our current work allows us to advance their application to water treatment and other fields of use as well as publish fundamental work on SPSs that have implications on very fundamental concepts, including electrolyte theory and the $\mathrm{H}$ bond. This work allows us to meet DOE science and applied missions.

\section{Publications}

Reimund, K., J. McCutcheon, F. Stewart, and A. Wilson, "Osmotic Energy Densities of Solutes and Solutions Applied to Pressure Retarded Osmosis," Journal of Membrane Science, in preparation.

McNally, J., and A. Wilson, "Density Functional Theory Analysis of Switchable Polarity Solvent (SPS) Structure-Function Model," Journal of Organic Chemistry, in preparation. 
Wilson, A., and C. Orme, "Concentration Dependent Speciation and Mass Transport Properties of Switchable Polarity Solvents," RCS Advances, Vol. 10, 7740-7751, 2015.

Wilson, A., and F. Stewart, "Structure-Function Study of Tertiary Amines as Switchable Polarity Solvents," RCS Advances, Vol. 4, 11039-11049, 2014.

\section{Presentations}

Wilson, A., and C. Orme, "Further Studies of Switchable Polarity Solvent (SPS) As Draw Solutes for Forward Osmosis," North American Membrane Society (NAMS) 24 th Annual Meeting, Houston, TX, June 2014.

Reimund, K., B. Coscia, A. Wilson, and J. McCutcheon, "Forward Osmosis Flux Performance and Membrane Compatibility Studies of the Dimethylcyclohexyl Ammonium Hydrogen Carbonate Switchable Polarity Solvent (SPS) Draw Solute," North American Membrane Society (NAMS) $24^{\text {th }}$ Annual Meeting, Houston, TX, June 2014. 


\title{
14-080 - Battery Material Characterization Technologies
}

\author{
Dennis C. Kunerth, Tim McJunkin, Dave Hurley, Eric Dufek, and Kevin Gering
}

Vital to the continued development of advanced battery technologies is the development of improved materials, material diagnostic tools, and methodologies to monitor battery components while in-service. An approach to developing diagnostic tools and in-service monitoring technologies is to adapt nondestructive evaluation methods (NDE) such as ultrasonics, eddy currents, or thermal wave imaging for use with battery materials, geometries, and service environments. Similar approaches have been used by INL researchers using ultrasonics to monitor ceramic sintering, eddy currents to detect cracking in coatings during high temperature thermal cycling, and visual/ultrasonic monitoring of weld processes for control and concurrent inspection. It is proposed that similar NDE methods can be developed to characterize battery materials and/or study the kinetics of in-service battery component degradation, thereby developing INL capability to support battery materials R\&D. Once developed and refined, these same sensing technologies can potentially be adapted for in-service battery health monitoring.

The technical objectives of the proposed research are threefold:

1. Determine feasibility via laboratory testing of selected NDE methods to detect and/or monitor battery material parameters such as porosity in real time (Year 1)

2. Develop diagnostic tools based on viable NDE methodologies into laboratory-based battery material R\&D capabilities (Years 2 and 3 )

3. Evaluate the viability of using the NDE methodologies for in-service battery diagnostics and management (Year 3).

Until recently, state-of-the-art electrode characterization was done in the destructive analysis realm, with indirect techniques evaluating the current and voltage profiles, or by monitoring the electrical properties of the battery through impedance measurement methods. If successful, the methods studied under this project will provide a more direct nondestructive evaluation of the battery's physical process, allowing better determination of failure modes and potentially enabling more detailed physical descriptions of electrochemical signatures to be identified. Identification of physical processes will provide systems models that are used to estimate battery state-of-health to better approximate the actual conditions within the battery. NDE approaches have been used to characterize battery materials but were primarily limited to neutron/x-ray radiography and neutron diffraction. Although these are useful functions that should not be ignored as laboratory-based research tools, these approaches are not suitable candidates for later in-service battery health determination and management.

Only one reference to the use of eddy currents to measure the state of charge in nickel (Ni) batteries via an electrode impedance method was found (Mancier, V. et al., "A Contact-less Method to Evaluate the State of Charge of Nickel Batteries Using Foucault's Eddy Currents," Journal of Power Sources, Vol. 117, Issue 1-2, 223232, May 15, 2003). However, in this article, it was noted that the "artifacts" associated with the impedance measurements may provide a means to detect electrode degradation. This points out the novelty and significance of the proposed approach. If successful, the NDE technologies to be evaluated (specifically ultrasonics and eddy currents) have the potential to be not only laboratory-based material interrogation technologies for R\&D but also developed into sensor technologies in the fabrication processes and for in-service battery health monitoring and management systems. 


\section{Summary}

The Year 1 objective of this LDRD was to determine the feasibility, via laboratory testing, of selected NDE methods to detect and/or monitor a battery material parameter such as porosity in real time. Ultrasonic, eddy current, and thermal-based techniques were evaluated. However, poor results from the initial thermal wave evaluation resulted in that approach being relegated to a low priority compared to the ultrasonic and eddy current approaches.

Ultrasonic interrogations rely on monitoring the mechanical and dimensional properties of the test piece. Ultrasonic measurements demonstrated the ability to detect the presence of the electrode as well as physical variations in electrode thickness. Figure 1 illustrates the use of laser-generated and laser-detected plate waves to interrogate the electrode material. Dispersion of the acoustic wave (change in velocity with frequency) is strongly influenced by the presence of the electrode material. Changes to the dispersion relationship caused by the presence of the film are noticeable for both high and low frequency components of the plate waveform.

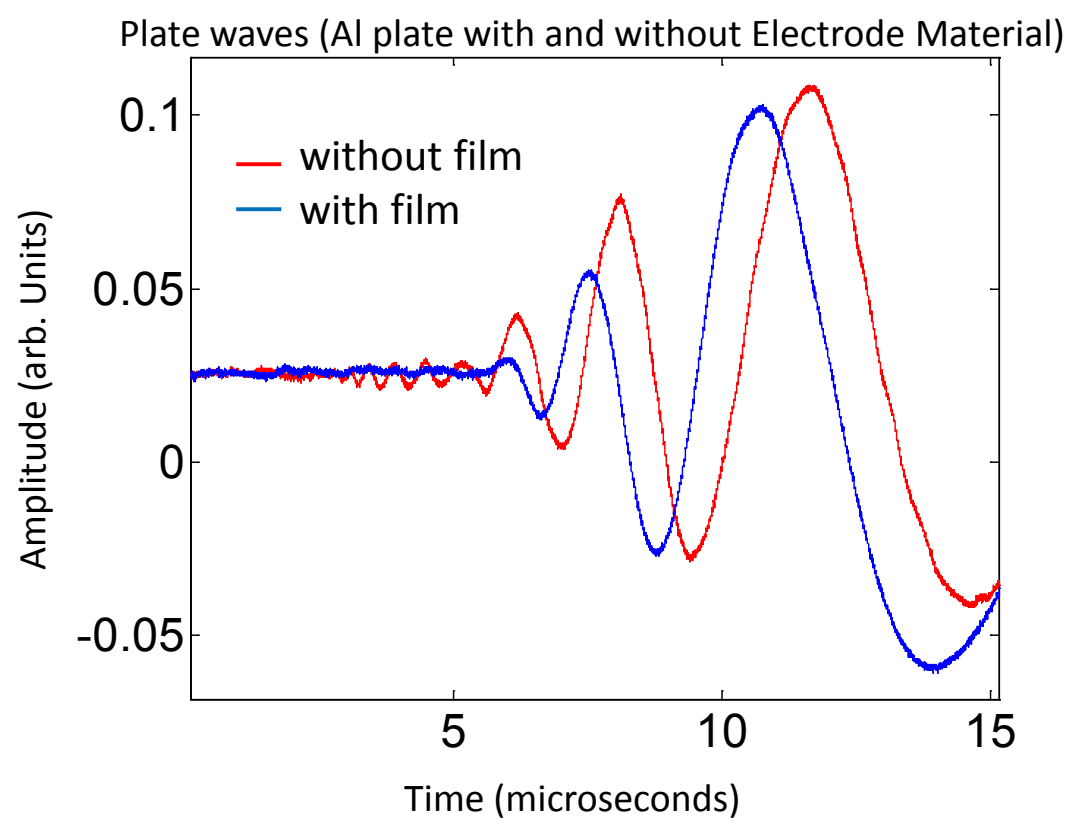

Figure 1. Laser-generated and laser-detected plate waves in aluminum (Al) substrate with and without electrode material.

Another measurement involving a test sample in which the thickness of the electrode film was altered by localized calendering demonstrates the ability of ultrasonics to detect physical changes in the composite electrode structure, see Figure 2. In Figure 2, the plate waves ( $\mathrm{pw}$ ) corresponding to test locations 1 and 2 exhibit similar dispersion relationships. This indicates that the electrode in these regions of the plate have similar thicknesses. However, the difference in dispersion relation between test locations 3 and 4 indicates that the electrodes have different thicknesses in these regions of the plate. This is significant in that it demonstrates the potential for ultrasonics to be used for monitoring industrial fabrication of electrode materials. Variations in signal amplitudes are a result of variable laser generation and detection, not electrode thickness. 


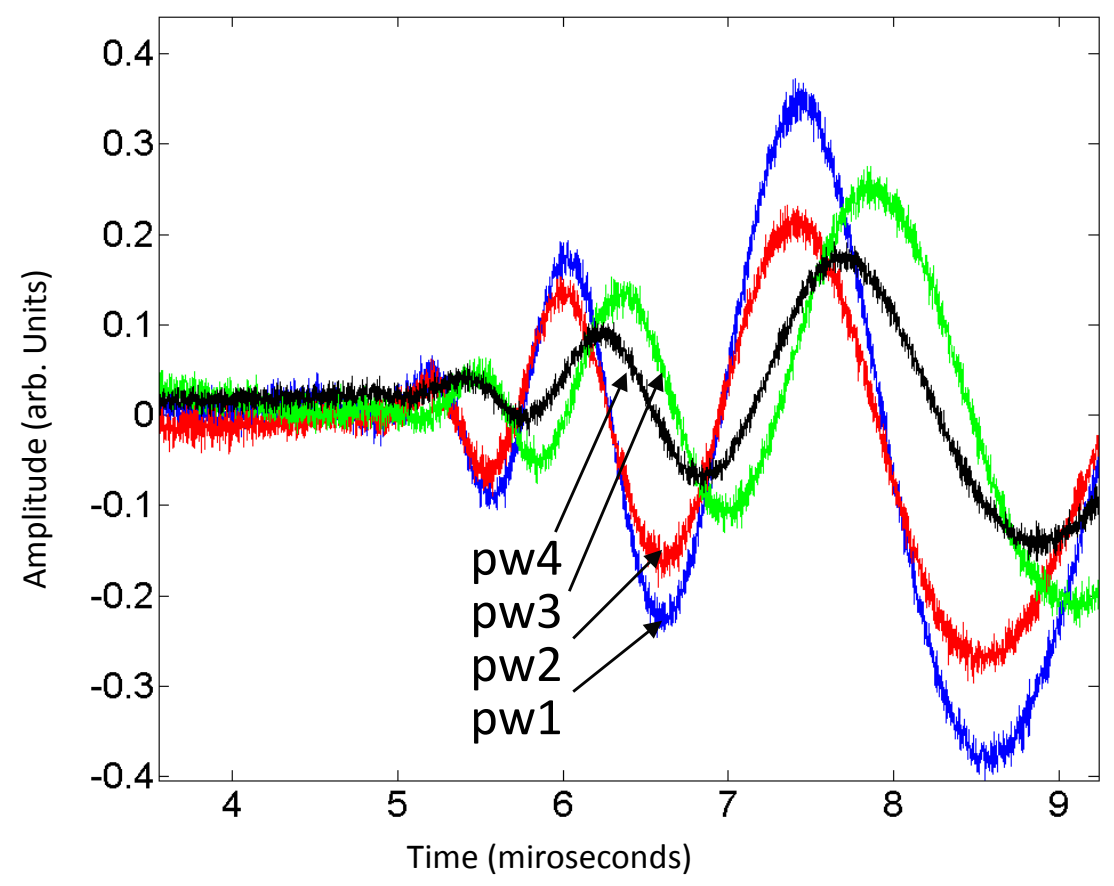

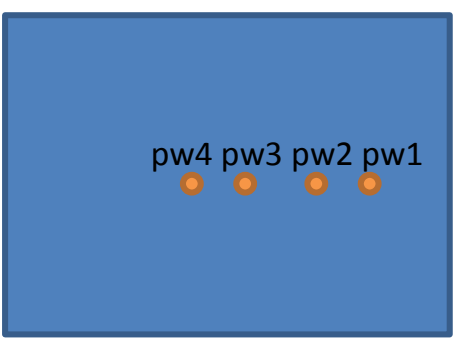

Test Locations on sample

Figure 2. Laser ultrasonic detection of electrode film thickness.

Eddy current interrogations rely on the electrical properties of the test piece. Initial testing was performed using off-the-shelf 2032 batteries (lithium [ $\mathrm{Li}] / \mathrm{MnO}_{2}$ chemistry) in which the batteries were discharged through a load to determine if eddy currents could detect changes in an active battery electrode. This battery was selected due to the relatively thick cathode structure compared to the much thinner electrodes encountered in the laboratory test samples. Observed were responses to both battery temperature variations due to current flow and internal material variation due to current production. Figure 3 provides an initial interpretation of the 2032 battery responses recorded for relatively low test frequencies that permitted eddy current generation through a significant portion, if not the full thickness, of the battery. Note that these measurements were performed through the metal battery case. The $25.5 \mathrm{ohm}$ load resulted in a higher current flow resulting in more resistive heating compared to the $123.0 \mathrm{ohm}$ load. Measurement time at load was different for the 25.5 and $123.0 \mathrm{ohm}$ tests (i.e., 1.5 versus 2.5 hours, respectively). Several minutes of data were collected after removal of the loads that resulted in "relaxation" of the battery components and a drop in temperature. 


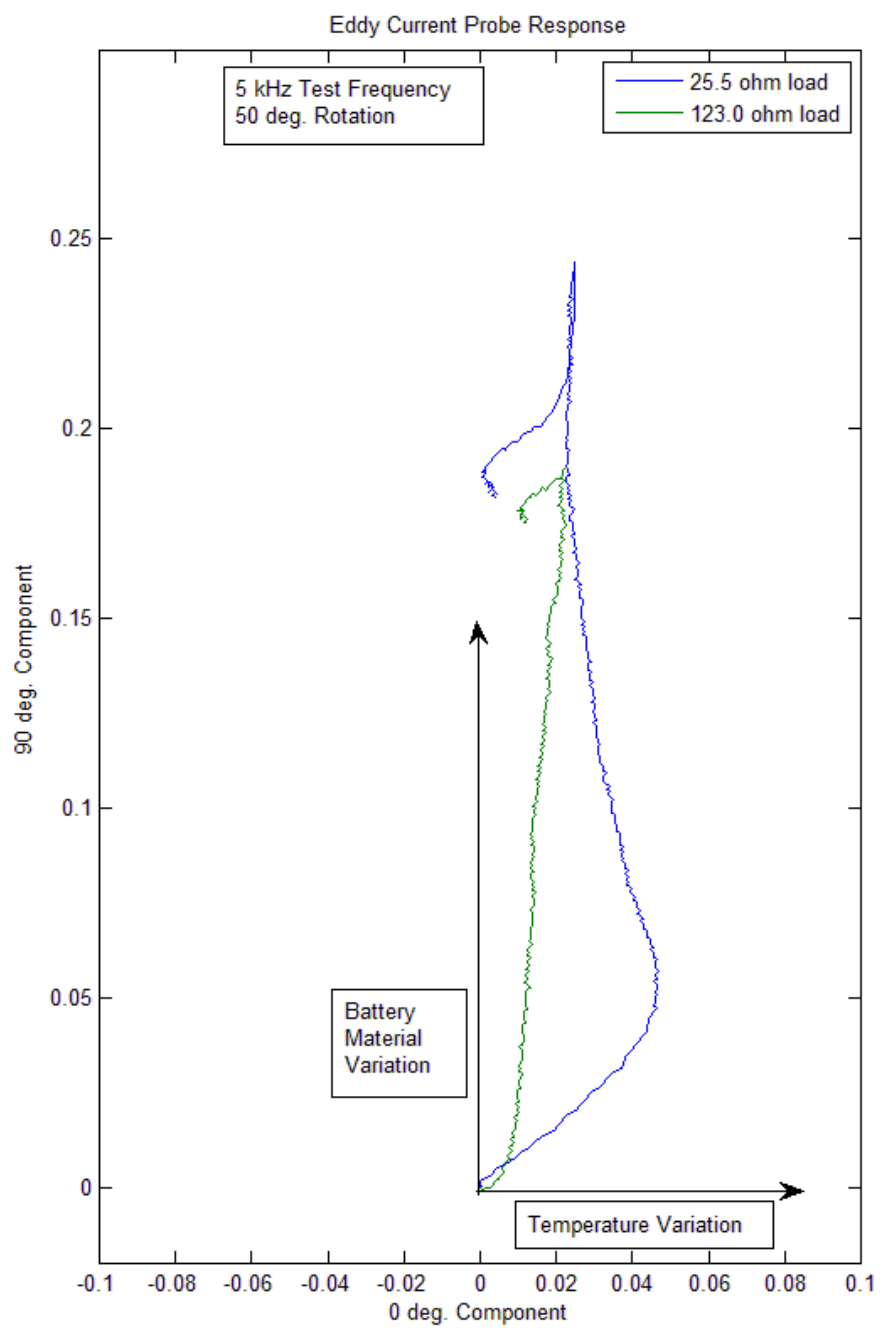

Figure 3. Eddy current responses recorded during loading of 2032 batteries.

Due to the nature of the commercial 2032 battery button cell construction, the material variation responses could not be specifically tied to a single physical material change in the battery. To correlate eddy current responses to battery material variations, an alternate battery configuration better suited for experimentation was selected. Spinel Li Ni MnO (LNMO) half-cell test samples were fabricated, and charge/discharge cycles were performed with eddy current monitoring. Figure 4 is the recorded eddy current response for a charge/discharge test. Plotted are the two eddy current signal components with time. 


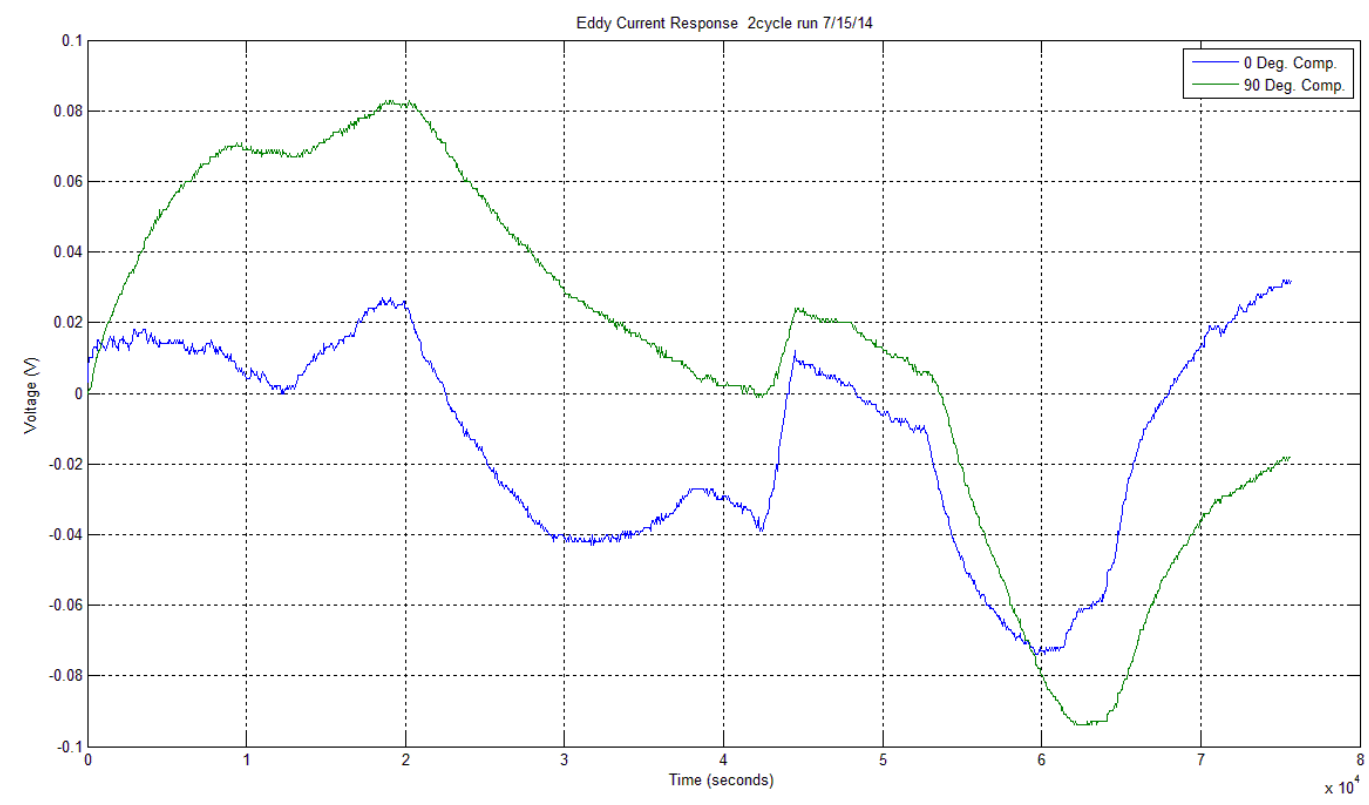

Figure 4. Eddy current response to charge/discharge cycles in a spinel LNMO half-cell.

The ability to use eddy currents and electrochemical signals to jointly probe a battery during cycling allows a more complete set of information to be obtained. The primary focus of early work for this project was to investigate the ability to obtain both sets of data in a parallel manner. During testing, no change in the open circuit voltage of a spinel LNMO-based half-cell was observed, signifying that the eddy currents were not perturbing the system. However, upon the application of a constant current to the LNMO half-cell, distinct signatures in the eddy current signal were observed which correlate with transitions occurring within the electrode material, see Figure 5. Follow-on work found that changes in temperature can be clearly observed using the combined methods. This is significant as eddy current analysis using different frequencies can alter the depth of the battery being investigated. As such, heat information, which is more directly related to the area of generation, could become available. This data would be a significant improvement over following the skin temperature of a cell, as is currently done, and would enable more advanced understanding of material performance and degradation. Current work is looking at more clearly making the distinction between heat generation and location and following how that changes with more extended cell cycling. 


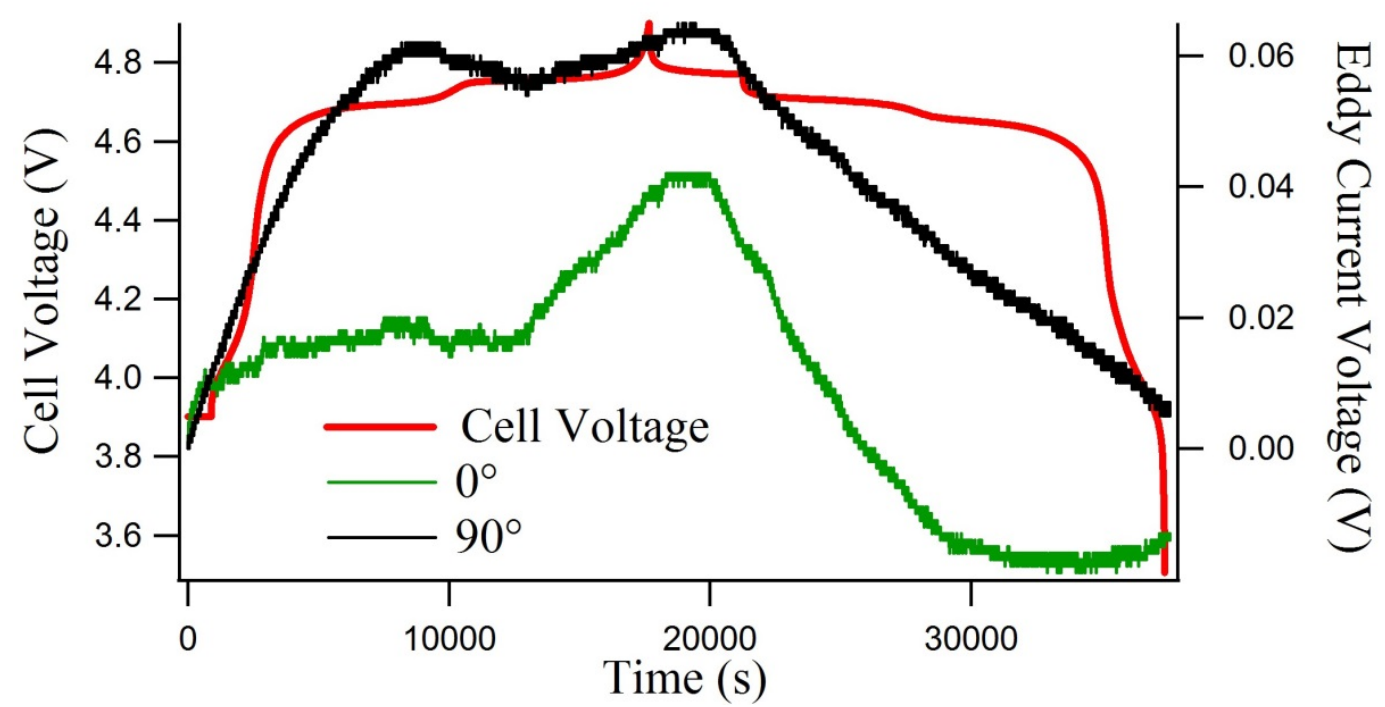

Figure 5. Correlation of eddy current response to material changes in LNMO half-cell.

\section{Benefits to DOE}

The goal of this LDRD is to develop R\&D diagnostic tools and health monitoring sensor technologies that will facilitate the development of advanced energy efficient battery technologies. This fits within the mission of the Office of Energy Efficiency and Renewable Energy, which aims to accelerate the development and facilitate the deployment of energy efficiency and renewable energy technologies and market-based solutions that strengthen U.S. energy security, environmental quality, and economic vitality. 


\title{
14-086 - Development of a Microgrid/Smartgrid Testbed for ESL and Super Lab Initiative with Load Variability Characterization and Control for Renewable Energy Integration
}

\author{
Kurt Myers, Jason Bush, Jake Gentle, Bob Turk, Tom Baldwin, and Rob Hovsapian
}

With increasing demand for renewable integration, the requirements of the legacy grid and market structures will rapidly exceed current capabilities. The ability to understand and model the system dynamics of real-world microgrids and distributed energy resources (DER) at multiple scales and geographic locations is essential to successfully increasing renewable penetration. The developing micro/smartgrid platform at INL addresses this issue through the development of DER grid testing and modeling capabilities in an integrated, collaborative environment. To achieve this goal, INL will design and construct an open-source smartgrid platform with integrated control system and component interaction R\&D, develop scalable grid models from acquired data, and work with industry to begin hardware and software integration testing for future microgrid and DER applications. This work will benefit the Department of Defense (DOD), utility, industry, and other partners through test and research input into their planning, development, business/economic dispatch, and operational models and processes. Additionally, this platform will allow direct testing and demonstration of various topologies of hardware (including smart inverters or other power electronics systems) and electrical, mechanical, and controls interactions, which may be difficult or impossible to accurately simulate when fully coupled to a larger microgrid or full utility grid system. The novelty of this research stems from its aim to determine the contribution of variability from renewable generation and various load types, and how management and control of grid systems can be improved through optimized integration of new and existing load, storage, and generation resource technologies, topics that have been identified by multiple potential industry partners as a major unanswered question in their systems. Furthermore, the proposed research supports the DOE goal of solving key technological challenges in making clean energy efficient and affordable while maintaining reliability and resiliency.

This work is providing INL with a state-of-the-art testbed for analyzing and dissecting the dynamic, coupled behavior of renewable generation, traditional generation, and load. Furthermore, the research into controllers for energy management schemes that provide user/grid security, cyber security, and enhanced grid economics will develop state-of-the-art capabilities/intellectual property) that can be shown as low-cost resources within well-designed electricity markets or microgrids.

\section{Summary}

Year 1 for the Microgrid/Smartgrid Research Testbed Development LDRD has been highly successful in meeting its objectives. Much of the Year 1 work has been focused on getting major pieces of the hardware, metering, and communications in place to enable Years 2 and 3 R\&D work to continue. Another major focus has been developing potential research collaborations with industry, utilities, university partners, and other federal customers and positioning the project to respond to external proposal and funding opportunities. For the latter, the project has been highly successful in being a part of a recent Advanced Research Projects Agency-Energy (ARPA-E) Cycling Hardware to Analyze and Ready Grid-Scale Electricity Storage (CHARGES) proposal for testing new battery technologies within a microgrid system and has developed several industry collaborative relationships that are leading to research and equipment involvement in development of the microgrid (Qnergy Concentrated Solar Power dishes donated, Keuka wind turbine and associated equipment, Schneider Electric for inverter and related systems testing, and interest from multiple utilities). 


\section{ENERGY \& ENVIRONMENT SCIENCE \& TECHNOLOGY}

Internal and external development and work/permit control processes have been achieved to enable installation and inspection of the first phases of equipment. We currently have in place $23 \mathrm{~kW}$ of solar photovoltaics (PV) (see Figure 1 below), five microinverters, one grid-tie string inverter, one on-off grid inverter/battery system, two smart inverters/four quadrant power converters, multiple electrical distribution panels, cabling and transformers, two secondary-use Li-ion battery systems, multiple metering systems, initial control and communication layers, and several controllable load connections and systems in place or in final development stages.

The project also has the Concentrated Solar Power and some of the wind turbine equipment on-site for installation completion in Years 2 and 3 and has a net-metering agreement in place with Idaho Falls Power to enable both grid-tie smartgrid interaction and island/microgrid mode research. The project has the initial Real Time Digital Simulator (RTDS) racks installed and associated equipment and modeling started to enable Hardware-in-the-Loop (HIL) and associated research in combination with other researchers. Coordination is also in place to connect the Nuclear Energy University Program industrial load/thermal loop research into the microgrid when it arrives later this year. This progress put INL in position to respond to multiple proposals including the ARPA-E CHARGES proposal for testing of new battery systems within microgrids, testing of Schneider Electric new inverters and smartgrid equipment, DOE Office of Electricity microgrid Funding Opportunity Announcement, and multiple other opportunities for collaboration with utilities, universities, and others. The project has utilized three interns and one post-doc this year and will continue to utilize university student and faculty collaborators as we move into the next phase of microgrid controls R\&D. Progress documentation and papers have been started, and communication with others is underway this fiscal year with presentations at upcoming Microgrid workshops and communications with regional utilities and universities for collaborative opportunities into next fiscal year.

Existing and developing university partnerships (interns, postdoctoral scholars, etc.) include: University of Idaho, Idaho State University, Florida State University, Washington State University, Colorado State University, Boise State University, University of Alaska Fairbanks, University of Calgary, Aalborg University Denmark, and Michigan Tech University.

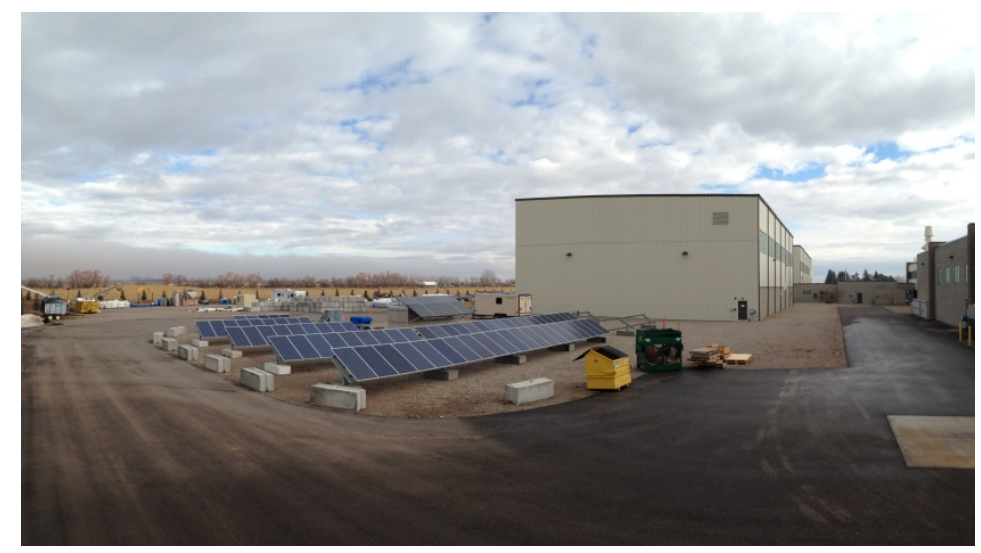

Figure 1. Portions of INL microgrid research assets. 


\section{Operational Research Concepts}

As this research project develops, INL will operate it as an inverter-based microgrid for research on high-penetration renewable power integration. Key components are over $13 \mathrm{~kW}$ of PV (to be expanded to $>30 \mathrm{~kW}$ ) in various arrays and interconnection technologies from microinverters to off/on-grid inverters. The off-grid capable PV array is paired with a $1568 \mathrm{Ah} / 24 \mathrm{VDC}$ lead-acid battery [flooded lead acid]. Two $2.4 \mathrm{~kW}$ wind turbines are also part of the grid, with expansion plans for a $50 \mathrm{~kW}$ turbine and the addition of several Concentrated Solar Power Stirling dish/generator systems. Additional energy storage devices within the distribution grid are a $10 \mathrm{~kW} / 18 \mathrm{kWh}$ Enerdel secondary-use Li-ion vehicle battery and a $100 \mathrm{~kW} / 80 \mathrm{kWh}$ secondary-use Li-ion battery with Princeton Power GTIB-100 kW inverter system.

The microgrid is primarily regulated through three different smart inverters, energy storage, system controller research developments, and load control. A Princeton Power microgrid site controller that can also provide onboard Battery Management System capabilities is also utilized. Thermal building systems (heating and cooling) are utilized for demand management applications, including an ice/chilled storage air conditioning system to provide peak shifting capabilities. For prolonged operation during unfavorable renewable energy availability, INL has future planning for the addition of fueled electric generation equipment (diesel, microturbine, Stirling, and/or fuel cell), combined heat-power, and fuel access/storage systems. While providing customer loads, the INL microgrid can be reconfigured as needed by temporarily re-routing power from other sources for some or all customers.

A Stirling engine, fuel cell, or other genset with multi-fuel capabilities coupled with the proper grid interface inverter (both on/off grid capable, with synchronizing, droop mode available, etc.), will allow for multiple research, development, and demonstration paths to occur at INL in conjunction with other activities such as biofuels development, grid integration, and smart grid modeling and application, hybrid energy systems, etc. Basic research operational concepts include:

- A primarily inverter-based microgrid for research, development, testing, and demonstration on high-penetration renewable power integration

- Inverter types, including grid-tie, on/off-grid, smart inverters, and microinverters

- Utilization of storage devices (both battery and thermal), controllable loads (heating, ventilating, and air conditioning; water heating; etc.), and electric vehicle charge control interactions that are key to the research concepts

- Multiple types of renewable and fueled generation integrated into a hybrid system, which can be used for research in grid interactive or islanded modes

- A platform for researching many utility and DOD/industry challenges with DER and microgrids. 


\section{ENERGY \& ENVIRONMENT SCIENCE \& TECHNOLOGY}

\section{Initial Research Areas}

- Interaction and capability differences for variation of arrangements with droop control, alternating current coupling, switching from maximum power point tracking/current-source to voltage-source/droop modes, and synching/switching transitions.

- Microgrid control system and algorithm development, integration, and testing, including utility interactive mode capabilities analysis. Includes protection and balancing of lower-inertia power systems/electronics.

- Dynamic, HIL, load flow, time series, and energy balance model developments for microgrids and smartgrid utility grid-tie interactions.

- Secondary-use battery, Battery Management System, storage, inverter/charger, and site controller integration challenges (key to being prepared for ARPA-E or other battery storage research and field application testing).

INL regularly collaborates with regional utilities on grid technologies research and demonstration, including areas such as transmission and distribution, renewable energy and smartgrid, voltage control, microgrids, cyber security, DER, and control. The microgrid LDRD project will enable deeper collaboration within utility partnerships. Historical partners include Idaho Power, Idaho Falls Power, Rocky Mountain Power, Utah Associated Municipal Power Systems, and others.

A major research focus of the project will be to construct, analyze, and model the characteristics of common, controllable loads that have the potential to significantly impact (positively or negatively) renewable energy integration. Understanding the scaling and expansion of distributed generation profiles is a significant research effort of this proposal and will build on the renewable energy assessment expertise and measurement data sets and techniques developed at INL and other national laboratories over many years. This project will couple the load characteristics obtained from the demonstration systems and other measurements with distributed generation microgrid characteristics, as well as regional weather geographical distribution effects on these systems, to generate expanded models of larger systems as inputs to economic and system dynamics models utilizing RTDS, Hybrid Optimization Model for Electric Renewable, GridLab-D, or other modeling systems to characterize impact potentials and costs.

\section{FY 2014 Activities and Accomplishments}

1. Created a dynamic RTDS microgrid model based on the well-accepted CERTS philosophy.

2. The RTDS-CERTS model has the capability of interfacing dynamic to perform HIL and controller HIL (CHIL) for future research.

a. This work resulted in the joint journal publication (ready for submission) between the INL Grid Integration Team and Washington State University. Title: "Study of CERTS Microgrid Resilience after V2G Integration."

3. Steady work progress with Keuka Wind, Inc., to transfer the Grid Integration Team researcher's operational wind turbine hardware lab set up from Wind Lab, Florida State University to ESL Power Systems, Inc., INL.

a. ABB converter and wind turbine generator are currently in the process of being integrated with the power system assets at ESL Power Systems, Inc. 


\section{ENERGY \& ENVIRONMENT SCIENCE \& TECHNOLOGY}

4. Interface development of an ABB power converter with the RTDS. This capability will eventually assist in research projects that necessitate a power HIL (PHIL) with the power converter.

5. Established a communication network simulation capability that integrates with the RTDS. This simulation box (based on Linux) is capable of simulating communication networks that assist in better power system operation and management. This capability will assist in future projects such as distributed RTDS simulations and coordination between geographically distributed microgrids. Conference paper based on this capability is currently under progress.

\section{Benefits to DOE}

This project is beneficial to DOE as it is developing smartgrid/microgrid and DER integration technologies and testing and modeling assets that will assist others in meeting federal goals for clean energy implementation, integration, and grid modernization. DOE is pursuing multiple efforts in DER, energy storage, grid modernization, and renewable energy integration, and this project is positioned to provide research and demonstration avenues across these areas in conjunction with linkages to utilities, industry, and DOD partnerships and efforts. The project has enabled INL to provide informative proposals to several DOE and DOD program areas and calls, including ARPA-E CHARGES, the Microgrid Funding Opportunity Announcement, and multiple DOD microgrid efforts.

\section{Publications}

Bush, J., K. Myers, and P. Hill, "Metering for Power Systems and Microgrid Planning," AWEA Windpower 2015, Orlando, FL, May 18-21, 2015.

Hill, P., J. Bush, and K. Myers, "Use of Wind Energy in INL and Other Microgrid Systems," AWEA Windpower 2015, Orlando, FL, May 18-21, 2015.

Myers, K., and J. Bush, "Field Test Results on INL Test of ZBB Battery for US Navy Hybrid Power System Applications," to be delivered to NAVFAC EXWC and ONR, September 2014.

Expanded controllable load concept paper developing from Bhattarai, B., et al., "Demand Flexibility from Residential Heat Pump" paper, IEEE PES General Meeting 2014. 


\title{
14-091 - Battery Health Estimation Based on Self-Discharge Fast Measurement and Soft Short-Circuit Detection
}

\author{
Sergiy Sazhin, (INL) Eric Dufek, Kevin Gering, and John Morrison ${ }^{1}$
}

Battery energy storage is destined to occupy a significant segment of our domestic energy strategy as it impacts electric drive vehicles, grid and micro-grid storage, and a growing number of consumer applications.

Lithium (Li)-ion cell chemistries are at the forefront of providing high-density energy storage. It is well known that Li-ion batteries may go into unpredictable catastrophic failures (CFs) due to their flammable components. The 2013 Boeing 787 Dreamliner fire event (Figure 1) at the Boston airport is a recent reminder. The primary reasons for the majority of Li-ion battery CFs are elevated self-discharge (SD) in one or more of the cells in the battery architecture and internal soft short-circuits (ISSCs) by dendrites. Conventional methods to measure SD are time-intensive (days), costly, and not feasible for on-board applications. ISSCs detection is a very complicated problem which has not been resolved.

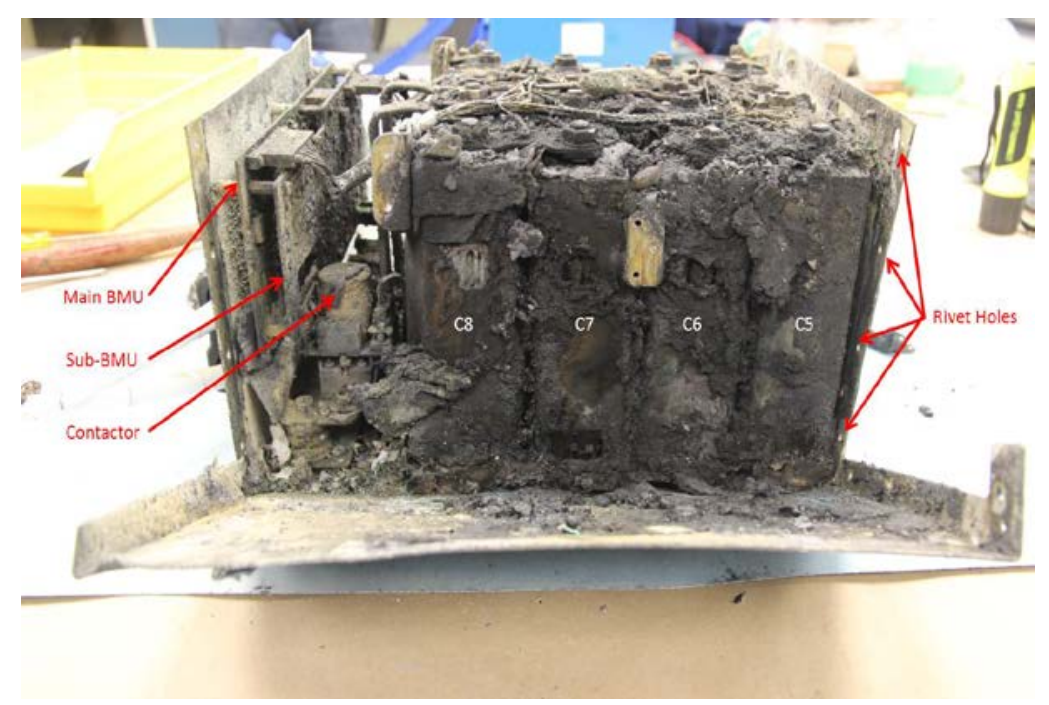

Figure 1. Burned out Li-ion battery from Boeing 787 Dreamliner.

This project aims to develop principally new diagnostic and prognostic methods that can be incorporated with a battery state of health (BSOH) sensor prototype for battery management systems, that is sensitive to unresolved concerns, and that can be used on electric vehicles and grid battery-based energy storage systems for prevention of CFs.

During FY 2014, the focus of this research was on developing a fast protocol for battery single cell SD measurement and ISSCs detection. Both experimental and modeling components were included at this early stage to obtain a more robust understanding of SD behavior. The FY 2015 plan assumes extension of the new approach for battery strings consisting of several cells. The SD measurement and detection of ISSCs is extremely important for the strings because any misbalance within the string will accelerate the chance of CF. The focus will be on estimating sensitivity of the method to detect abnormal performance of the string consisting of a

\footnotetext{
${ }^{1}$ Montana Tech of the University of Montana
} 
non-healthy cell. Collaborators from Montana Tech of the University of Montana will be involved with the aim to conceive a BSOH sensor prototype based on the INL detection method. The FY 2016 plan assumes more intense involvement of Montana Tech of University Montana for development of an on-board BSOH sensor prototype. During the prototype development, devices will be constructed and initially evaluated at Montana Tech of University Montana with more rigorous evaluation occurring at INL.

\section{Summary}

The aim of FY 2014 (Year 1) was to develop experimental test protocol for fast SD measurement for a single battery cell. Such a protocol is a basis for detection of dendrites that cause ISSCs and undermine battery safety. Several paths were planned. The first, most important, and most challenging path was to find a compromise between measurement sensitivity and measurement time with the aim to reduce the time of measurement and satisfy the practical need of fast on-board sensors. The challenges were very small, noisy, and sometimes not reproducible signals; long measurement times ( $>12 \mathrm{~h}$ in our initial pre-proposal approach, and days required for conventional measurement). The challenges were met with a faster than anticipated test procedure that allows SD measurements in 2-3 h. The main research findings are as follows. It was found that SD is a nonlinear function of time; therefore, the proposed fast method to economically measure actual SD in-time values is justified. This is especially true for intermittent ISSCs phenomena. Commercial wide-purpose instruments are made with temperature-sensitive hardware that contributes to measurement noise and poor reproducibility. The FY 2015 (Year 2) and 2016 (Year 3) plans for the development of a specialized sensor used specifically for an accurate SD measurement, as laid out in the initial LDRD plan, are therefore distinctly justified. As part of the method development, effort was placed in developing a model that physically describes SD. As can be seen in Figure 2, a physically relevant model (solid lines) has been developed, which effectively describes experimental behavior over multiple runs. When the modeling approach is used in tandem with the test protocol, it is feasible to determine meaningful SD attributes from only a few minutes of measurements. In addition to modeling efforts, future work in FY 2015 and FY 2016 will combine the developed fast SD method with other state-of-health diagnostic methods to more fully enable true diagnostic and predictive capabilities. The work will also expand to include arbitrary use conditions, such as temperature, state-of-charge, and aging, to produce a robust and unique tool for industrial relevance.

\section{Benefits to DOE}

The technical relevance and novelty of the work lies in a completely new capability to monitor BSOH on a regular basis with the ability to detect early battery malfunction, prevent CFs, and predict battery life. Current state-of-the-art does not allow this. The Year 1 work put a solid basis for success.

The work benefits several DOE missions. For Energy and National Security, CF events will be eliminated, and battery service life will be cost-effectively increased. This will promote penetration of Li-ion batteries into the electric vehicles and grid markets, including reduction in anxiety among battery end users. Electrification of the vehicle fleet and increased battery-based energy storage will lead to less demand for foreign energy sources providing more national security. The CFs are generally very harmful for environment and costly to cleanup. For Enviromental Quality, the elimination of battery failures and increase in performance of energy storage systems will lead to an overall increase in energy efficiency and reduced need for fossil energy, which reduces in turn the generation of green house gases. For Science and Technology, advanced diagnostic methods for energy storage applications will aid in advancing the mechanistic-level scientific understanding of the processes played in battery systems architectures. Development of an on-board SD sensor for battery management will raise technology of energy storage systems to another level of state-of-the-art and safety, and will lower risk perceptions. Advanced 
understanding of battery safety also falls within the mission of the National Highway Traffic and Safety Administration within the Department of Transportation. Additionally, branches within the Department of Defense would certainly gain benefit from this capability applied to Unmounted Soldier applications and critical forward-command energy storage scenarios. Also, the method can be used at battery production for premature screening and preventing potentially faulty batteries going to the market.

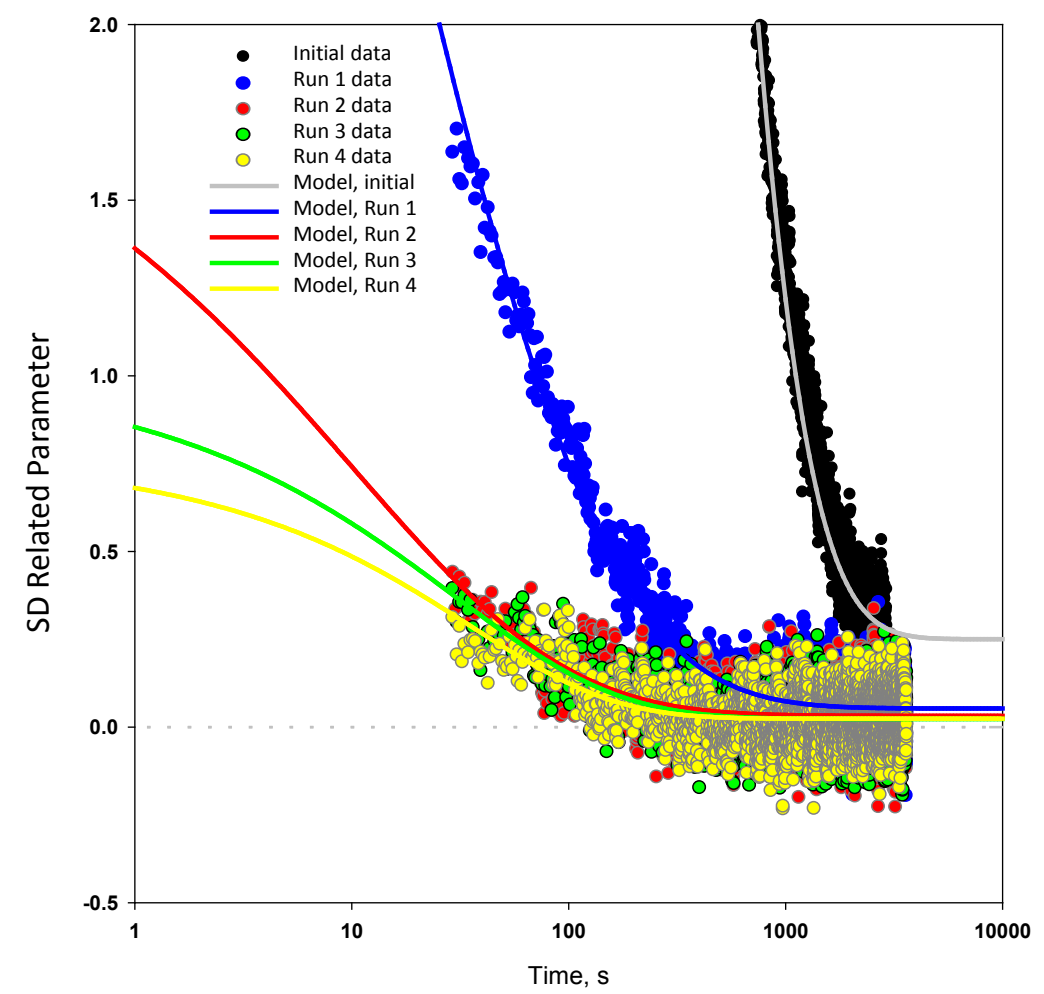

Figure 2. Experimental data (symbols) and modeling results (solid lines) for SD behavior of a commercial Li-ion cell.

\section{Publications and presentations}

Based on Year 1 results, it is expected that peer reviewed publications and presentations will be generated mid-year during FY 2015 and in FY 2016, as originally scheduled. 


\title{
14-095 - In Situ Measurement of Electrolyte Chemistry in Battery Cells During Operation
}

\author{
Gary S. Groenewold, Kevin Gering, David Jamison, and Chris A. Zarzana
}

Lithium ( $\mathrm{Li}$ ) ion batteries are remarkable power storage devices capable of delivering power over repeated discharge-recharge cycles and a range of environmental conditions. Nevertheless, Li ion battery performance is eventually degraded as a result of chemical and physical changes within the cells. If time- and use-dependent behavior were known, it is likely that informed approaches to mitigate deterioration and improve performance would emerge. Unfortunately, battery cells are sealed to prevent reaction with the ambient atmosphere, so it is difficult to measure quantitative chemical changes occurring within the cell in situ. Recently, we have developed an analysis approach that enables acquisition of reproducible mass spectra from nanoliter samples. If a minute volume could be harvested from the electrolyte

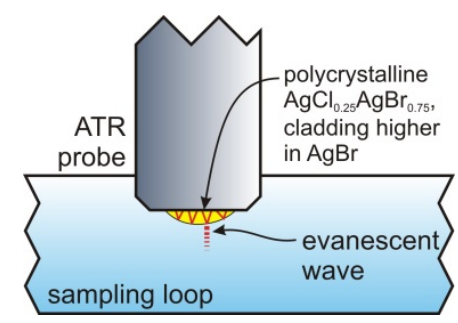

Figure 1. ATR-IR probe, evanescent wave penetrating the fluid sample. fluids within a $\mathrm{Li}$ ion battery without compromising battery function, it is probable that chemical changes could be measured as the battery undergoes repetitive discharge-recharge cycles. Additionally, insertion of a miniature infrared (IR) probe that operates on the principle of attenuated total reflectance (ATR) would enable measurement of the IR spectra of the carbonate electrolytes, which have intense carbon (C)-oxygen (O) bond stretching vibrations in the IR spectrum. ATR functions by passing IR light into a light-transmitting fiber having an index of refraction greater than the sample, which is in contact with the fiber (Figure 1). When the angle of incidence is sufficiently high, the light will be totally internally reflected, which occurs with propagation of an evanescent wave a short distance into the sample and results in absorption if the frequency matches that of a bond vibration. The carbonates have intense $\mathrm{C}-\mathrm{O}$ vibrations that are sensitive to degradation reactions and $\mathrm{Li}$ complexation, and so the approach is expected to provide new means for interrogating changes in the electrolyte chemistry.

We hypothesize that test cells incorporating a micro-ATR probe and a nanoliter sampling port can be designed for analysis of the electrolyte fluid and, further, that repeated nanoliter samples can be collected during battery operation to provide insight into the chemical changes occurring as the battery is used. We will fabricate cells with nanoliter sampling ports for the mass spectrometric analyses and also with optical ports that will enable complementary, real-time vibrational spectroscopy analyses. Currently there are no test cells compatible with ongoing, real-time chemical analysis, which indicates that the research would provide a significant advance in the state-of-the-art of battery research.

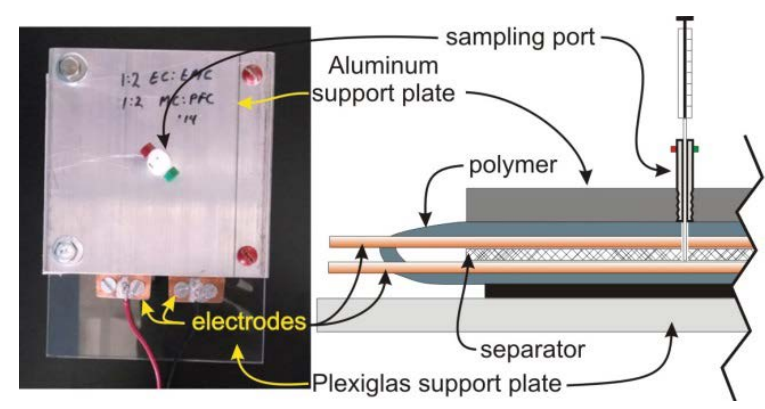

Figure 2. Photo and schematic diagram of the initial prototype of the pouch cell.

\section{Summary}

Critical research activities during FY 2014 were the design and fabrication of a test cell and measurement devices. First-generation test cells were designed and fabricated that will support acquisition of detailed chemical analysis without perturbing battery operation (Figure 2). A simplified pouch cell design was adopted that would 
accommodate a Teflon sampling port and a fitting that would hold an ATR IR probe. At the end of FY 2014, the cell was in the assembly phase, with different glue materials being tested for their ability to adhere the polymeric pouch material to the support plates. The electrode and separator assemblies were also completed and sampling ports procured. Initial charge/recharge profiles validated the cell's function (Figure 3).

An exceptionally small ATR probe was specified, and a vendor (ArtPhotonics) was contracted for fabrication; the probe was delivered during the last week of FY 2014 and will be validated during FY 2015. The diameter of the probe is $3 \mathrm{~mm}$ (Figure 4) and capped with a silicon window that protects an $\mathrm{AgCl}-\mathrm{AgBr}$ fiber, which has high transmission over the salient range of IR frequencies. In addition, mass spectrometry approaches based on analysis of nanoliter samples were refined for measurement of electrolyte degradation chemistry.

\section{Benefits to DOE}

The project holds excellent potential for providing a much improved understanding of electrolyte chemistry, specifically identification of reaction products formed by electrolysis of the carbonates.

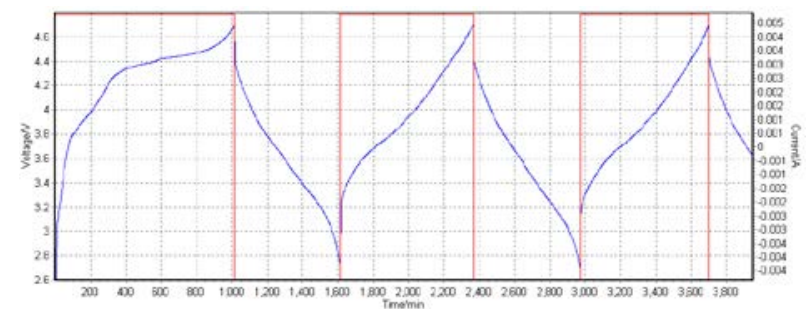

Figure 3. Charge/discharge of the pouch cell. Red, applied current; blue, voltage profile. Initial charge profile indicates formation of the SEl.

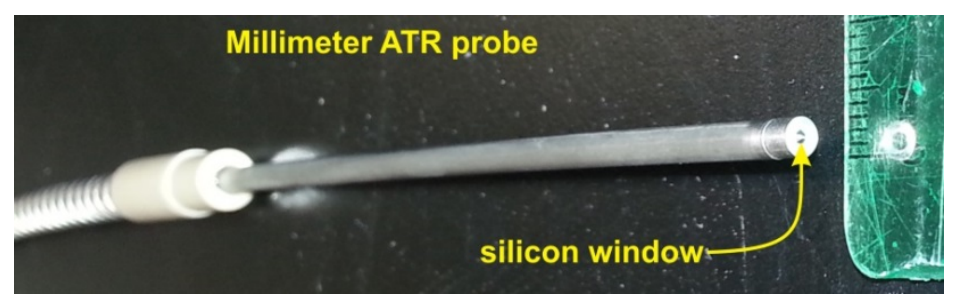

Figure 4. Photograph of the miniature ATR-IR probe. The measurement technology should open new strategies for examining the surface electrolyte interface, gas formation, changes in the available $\mathrm{Li}^{+}$inventory, and approaches for looking at temperature-induced phenomena that affect cell performance. Consequent improvements in battery technology would have wide-reaching effects benefitting virtually all government agencies. 


\title{
14-106 - Understanding the Growth of Ultra-Long Carbon Nanotubes
}

\author{
Joshua J. Kane and Robert V. Fox
}

The carbon nanotube (CNT) has some extraordinary properties, including a tensile strength one hundred times greater than steel, a thermal conductivity rivaled only by the purest of diamonds, and an electrical conductivity similar to copper $(\mathrm{Cu})$ but capable of carrying significantly higher current densities. These properties make it a desirable material for future composites, coatings and films, energy storage, and environmental applications.

Current CNT applications are not able to take full advantage of these unique properties due to the inability to grow aligned arrays of macroscopic length CNTs. A majority of industrial applications currently use a random dispersion of CNTs within a bulk material to slightly augment the material properties of the composite.

The main factor limiting the growth of CNTs is the poisoning and eventual deactivation of the catalyst. The purpose of this project is to understand the factors affecting the deactivation kinetics at a continuum level. Once the kinetics are understood, a solution can be proposed to prolong growth or potentially eliminate deactivation altogether. Factors such as initial catalyst particle size, catalyst substrate, and the gas composition will be varied to help understand their role in the deactivation kinetics.

\section{Summary}

Three major achievements were made in FY 2014. The first was the design, acquisition, and receipt of an experimental system capable of measuring the rate of carbon (C) accumulation within a chemical vapor deposition chamber. This was accomplished by the end of July 2014. The second achievement was the setup of the gas analysis equipment and routing of gas lines through the chemical vapor deposition furnace and gas analyzer. This was achieved on September 30, 2014.

The final achievement was more scientific in nature. Iron (Fe) oxide nano-catalysts were synthesized at INL on a $\mathrm{SiO}_{2}$ substrate and analyzed via atomic force microscopy at Boise State University. The image shown in Figure $1 \mathrm{~A}$ represents a synthesis route resulting in a nanoparticle density of nanoparticles dispersed on average less than $30 \mathrm{~nm}$ apart. The histogram in Figure 1B corresponds to Figure 1A. The height distribution appears to be bimodal. In FY 2015, the synthesis route will be modified to achieve a single peak with a narrow particle size range. 


\section{ENERGY \& ENVIRONMENT SCIENCE \& TECHNOLOGY}
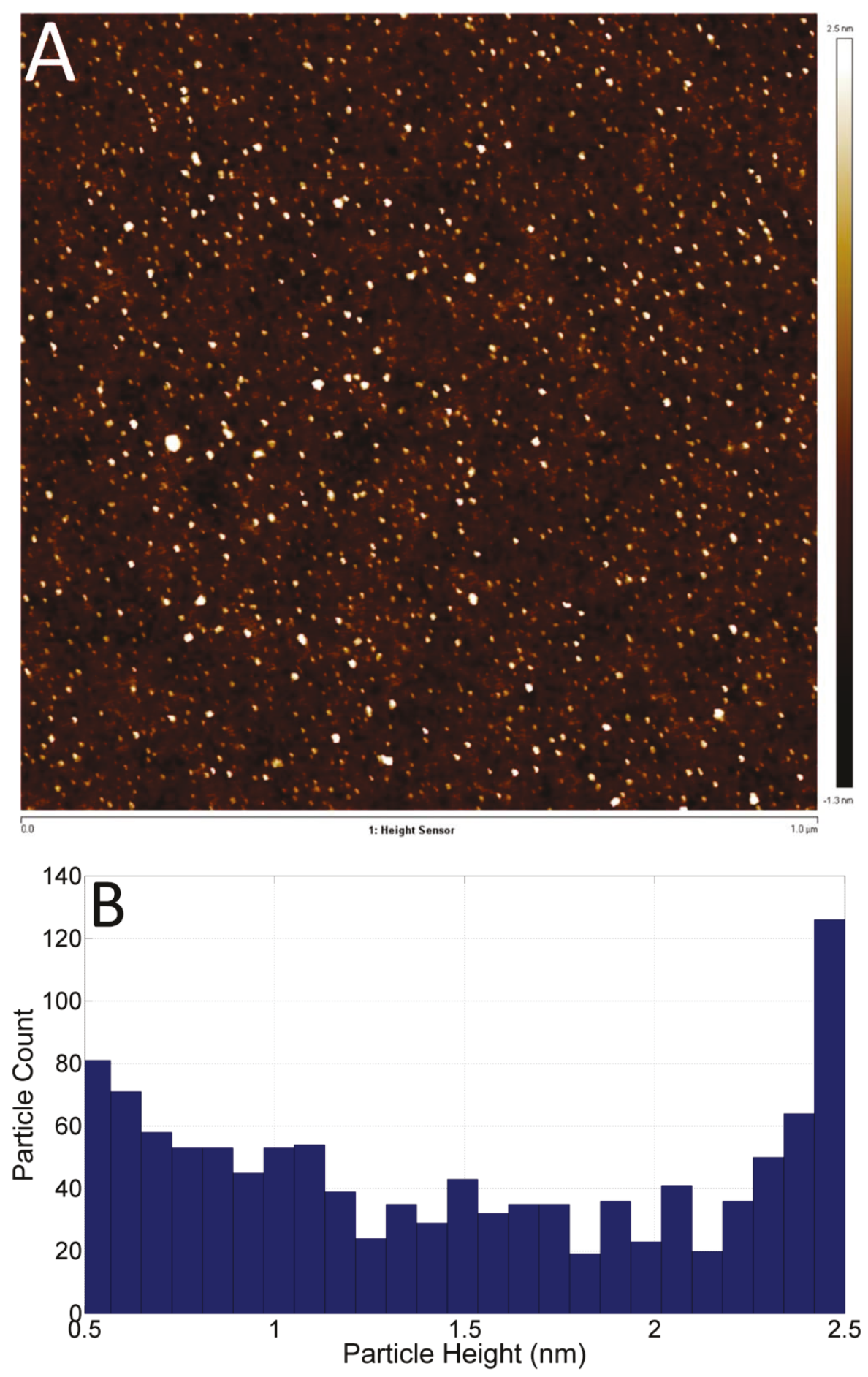

Figure 1. (A) Representation of a synthesis route resulting in a nanoparticle density of nanoparticles dispersed on average less than $30 \mathrm{~nm}$ apart. (B) Bimodal height distribution of the nanoparticles in (A). 


\section{Benefits to DOE}

The mission of DOE is to ensure security and prosperity in the United States through transformative science and technological solutions addressing energy, nuclear, and environmental needs. The current project will promote achievement of this national security mission by enabling the development of high performance materials using aligned arrays of ultra-long CNTs. Potential applications include a lighter and more flexible electrical wire with potential for higher current densities, low pressure differential filtrations systems for biological contaminants, and advanced alloys and composites with potential applications for nuclear and other extreme environment industries.

\section{Publications}

Publications have not been written to date on the work accomplished. It is a major goal of the principal investigator to publish one relevant publication regarding this work by the end of FY 2015. Carbon appears to be a good place for dissemination of scientific accomplishments related to CNTs. 


\section{INSTITUTE FOR

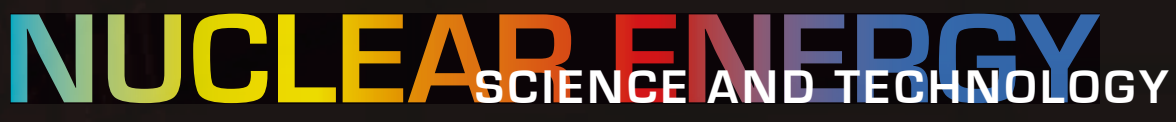




\section{2-026 - Protectiveness and Stability of the Zirconium Oxide in Early-Phase Corrosion of Zirconium Alloys - Predictive Relations to Surface Structure and Composition}

Xianming Bai and Bilge Yildiz ${ }^{1}$

Zirconium ( $\mathrm{Zr}$ ) alloys are widely used as cladding materials in light water reactors. However, water-side corrosion common in these alloys can lead to serious cladding failure. The corrosion depends on many factors such as microstructural features, alloy elements, surface chemistry, stresses, and radiation. To selectively design materials to suppress corrosion, a fundamental understanding of each unit factor is critical. This project aims at gaining a better understanding of these unit processes at the atomic level using integrated, state-of-the-art experimental and computational approaches. Four main controlling parameters are investigated-the alloying element, surface chemistry, surface microstructure, and stress.

\section{Summary}

To study the effects of niobium (Nb) on corrosion kinetics, we performed in situ, angle resolved x-ray photoelectron spectroscopy (XPS) and synchrotron XPS on both pure $\mathrm{Zr}$ and a Zr-2.5\%Nb alloy, characterizing the properties of the surface oxide. We found that a more stoichiometric $Z r$ oxide layer is formed on the $\mathrm{Zr}-2.5 \% \mathrm{Nb}$ than on the pure $\mathrm{Zr}$, indicating that $\mathrm{Nb}$ reduces the oxygen vacancy concentration and slows down oxygen transport, thereby providing better corrosion resistance. The chemical content analysis showed $\mathrm{Nb}$ segregation near the oxide surface during oxidation (Figure 1(a)). Complimentary density functional theory-based calculations were performed to study the impact of substitutional $\mathrm{Nb}$ on the defect equilibria of tetragonal $\mathrm{ZrO}_{2}$ $\left(\mathrm{t}-\mathrm{ZrO}_{2}\right)$ at $1500^{\circ} \mathrm{C}$ (Figure 1). Consistent with our experiments (Figure 1(a)), Nb tends to segregate to oxygen-rich regions such as the oxide surface. $\mathrm{Nb}$-doped $\mathrm{t}-\mathrm{ZrO}_{2}$ has two to three times more free electrons and five times less oxygen vacancies than pure $\mathrm{Zr}$. These $\mathrm{Nb}$-induced changes may result in competing effects on corrosion resistance and hydrogen pickup. The charge states of $\mathrm{Zr}$ at an oxidized $\mathrm{Zr}(0001)$ surface at $300 \mathrm{~K}$ is analyzed using synchrotron XPS (Figure 1(c)). The data directly demonstrated for the first time the formation of $\mathrm{Zr}$ sub-valence states during oxidation, answering a long-debated question of whether $\mathrm{Zr} 1+, 2+$, and 3+ charge states are able to form during oxidation (Figure 1(d)). By applying an electric field that mimics the corrosion-induced Mott potential, we found that a shoulder shows up in the XPS spectrum, indicating that the electric field assists the formation of $\mathrm{Zr} 2+$ and $3+$ charge states.

\footnotetext{
${ }^{1}$ Massachusetts Institute of Technology
} 


\section{INSTITUTE FOR NUCLEAR ENERGY SCIENCE \& TECHNOLOGY}
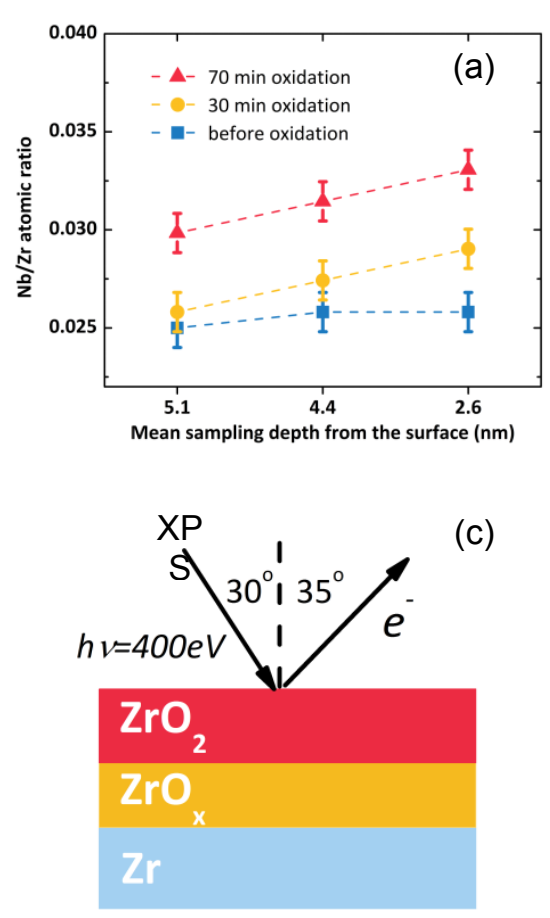
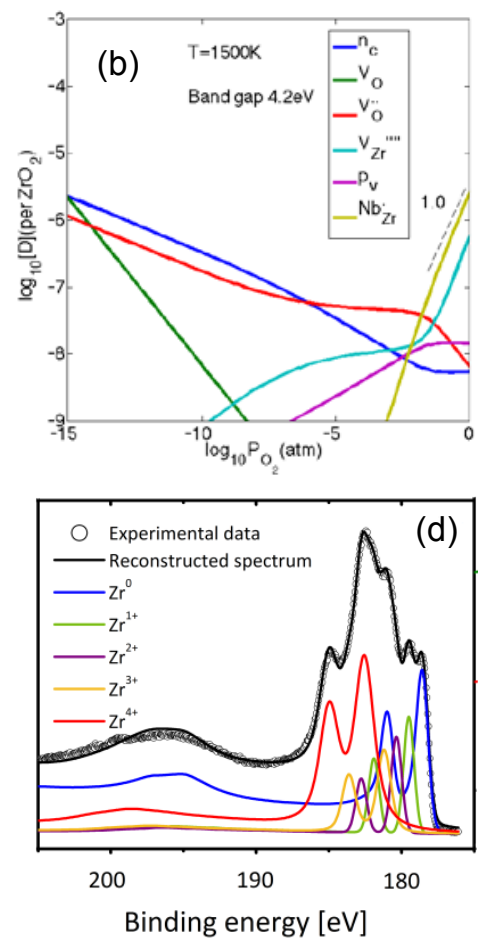

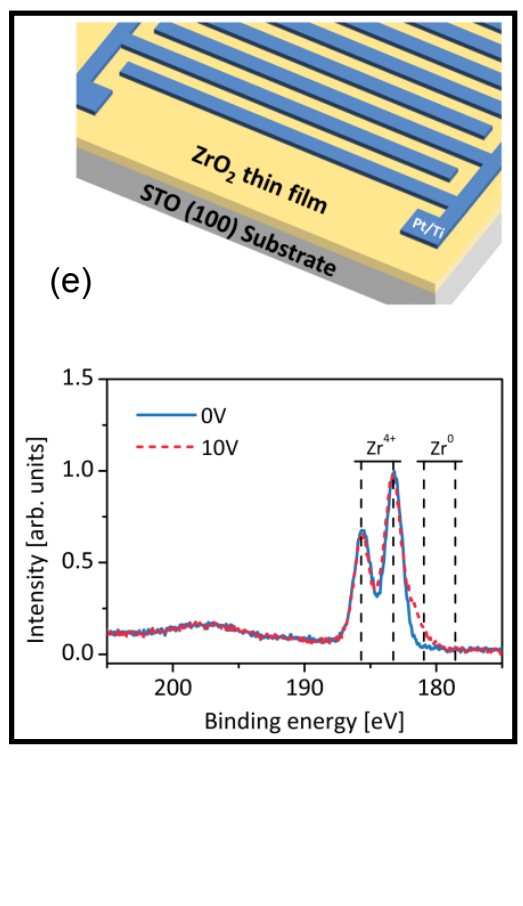

Figure 1. The effects of $\mathrm{Nb}$ and surface chemistry on oxidation in $\mathrm{Zr}$ and $\mathrm{Zr}$ alloys: (a) angle resolved XPS measurement of $\mathrm{Nb} / \mathrm{Zr}$ ratio in the surface oxide, (b) density functional theory calculation of Kröger-Vink diagram of the point defects in a $\mathrm{Nb}$-doped $t-\mathrm{ZrO}_{2}$ at $1500 \mathrm{~K}$, (c) schematic of characterizing surface oxide chemistry using XPS, (d) Zr 3d XPS spectrum, and (e) effect of electrical field (Mott potential) on oxide chemistry.

To study oxygen transport in $\mathrm{t}-\mathrm{ZrO}_{2}$, which is a possible rate limiting step of corrosion kinetics, temperature accelerated dynamics and molecular dynamics simulations were conducted to study oxygen defect diffusion under different conditions. Our results show that oxygen diffusion in $\mathrm{t}-\mathrm{ZrO}_{2}$ is anisotropic and different diffusion paths exist (Figure 2(a)). When a $\mathrm{Zr}$ metal transforms to a $\mathrm{ZrO}_{2}$, the volume expansion is about $56 \%$, which induces significant stresses. We found that under compressive strains, the oxygen diffusivities decrease significantly. At small tensile strains, the diffusivities increase moderately. At large tensile strains, the diffusivities decrease again (Figure 2(b)). These results suggest that applying external compressive stresses may help improve the corrosion resistance. The effects of microstructural features, such as grain boundaries (GBs), on oxygen transport were also studied. Compared with a bulk oxide, the oxygen defect migration energies at three GBs are much higher (Figure 2(c)), suggesting that GBs may not be fast oxygen transport paths, which is counter-intuitive. For cation diffusion, GB diffusion is also slower. These results suggest that we may tune the stress state and microstructures to achieve better corrosion resistance. 


\section{INSTITUTE FOR NUCLEAR ENERGY SCIENCE \& TECHNOLOGY}
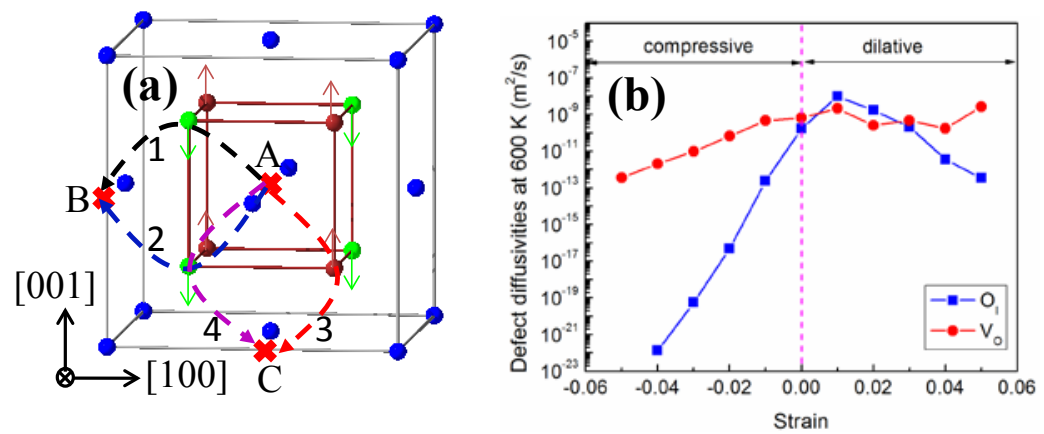

(c) \begin{tabular}{|l|c|c|}
\hline & \multicolumn{3}{|c|}{ Defect migration barriers $(\mathrm{eV})$} \\
\hline & $\mathrm{O}_{\mathrm{I}}$ & $\mathrm{V}_{\mathrm{O}}$ \\
\hline Bulk & $\mathbf{0 . 5 4}$ & $\mathbf{0 . 2 9}$ \\
\hline GB 1 & 0.84 & 0.64 \\
\hline GB 2 & 0.77 & 0.83 \\
\hline GB 3 & too slow & too slow \\
\hline
\end{tabular}

Figure 2. Temperature accelerated dynamics and molecular dynamics modeling of oxygen defect diffusion in $t-\mathrm{ZrO}_{2}$ : (a) anisotropic oxygen interstitial migration in bulk oxide, (b) strain effects on oxygen defect diffusivities, and (c) comparison of the oxygen defect migration barrier in bulk and in a GB.

\section{Benefits to DOE}

Our work will contribute to DOE's leading role on basic science research of materials behavior and performance in extreme environments. This work will help guide the design of corrosion-resistant $\mathrm{Zr}$ alloys for cladding and improve nuclear energy safety and economics while reducing the carbon footprint of the energy sector.

\section{Publications}

Ma, W., F. Herbert, and B. Yildiz, "Nonequilibrium Oxidation States in Zirconium Oxide during Early Oxidation of Zirconium," Phys. Rev. Lett., (submitted).

Otgonbaatar, U., M. Youssef, W. Ma, and B. Yildiz, "Effect of Niobium on the Defect Chemistry and Oxidation Kinetics of Tetragonal ZrO2," J. Phys. Chem. C, Vol. 118, 20122-20131, 2014.

Bai, X., Y. Zhang, and M. Tonks, "Computer Modeling of Transport of Oxidizing Species in Grain Boundaries during Zirconium Corrosion,” T. Am. Nucl. Soc., Vol. 110, 980-3, 2014.

Bai, X., Y. Zhang, and M. Tonks, "Atomistic Studies of Cation Transport in Tetragonal $\mathrm{ZrO}_{2}$ During Zirconium Corrosion," Proceedings for the Third International Workshop on Structural Materials for Innovative Nuclear Systems, Idaho Falls, ID, October 7-10, 2013.

Bai, X., Y. Zhang, and M. Tonks, "Strain Effects on Oxygen Transport in Tetragonal Zirconium Dioxide," Phys. Chem. Chem. Phys., Vol. 15, 19438-19449, 2013.

Otgonbaatar, U., The Effect of Niobium on the Defect Chemistry and Corrosion Kinetics of Tetragonal ZrO ${ }_{2}: \mathrm{A}$ Density Functional Theory Study, M.S. Thesis, Massachusetts Institute of Technology, 2013. 


\section{Presentations}

Yildiz, B., "Uncovering the Inner Workings of Metal Corrosion by Combining Surface Sensitive Experiments and Multiscale Modeling," MPC Materials Day Symposium, Massachusetts Institute of Technology, Cambridge, MA, October 2014.

Bai, X., Y. Zhang, and M. Tonks, "Computer Modeling of Transport of Oxidizing Species in Grain Boundaries During Zirconium Corrosion," American Nuclear Society Annual Meeting, Reno, NV, June 2014.

Youssef, M., W. Ma, W. Herbert, and B. Yildiz, "Mechanisms of Zr Oxidation and Hydrogen Absorption through $\mathrm{ZrO}_{2}$," Nuclear Engineering Colloquium, University of Illinois at Urbana-Champaign, IL, March 2014.

Ma, W., U. Otgonbaatar, M. Youssef, W. Herbert, and B. Yildiz, "Initial Oxidation Kinetics of Single Crystal Zirconium and Zirconium-Niobium Alloys," 2013 Materials Research Society Fall Meeting, Boston, MA, December 2013.

Otgonbaatar, U., M. Youssef, and B. Yildiz, "Effect of Niobium on the Defect Chemistry and Corrosion Kinetics of Tetragonal $\mathrm{ZrO}_{2}$," Third International Workshop on Structural Materials for Innovative Nuclear Systems, Idaho Falls, ID, October 2013.

Ma, W., et al., "Effect of Niobium on the Initial Oxidation of Zirconium Based Alloys," Third International Workshop on Structural Materials for Innovative Nuclear Systems, Idaho Falls, ID, October 2013.

Bai, X., "Atomistic Studies of the Effects of Strain and Grain Boundary Character on Oxygen Transport during Zirconium Corrosion," Third International Workshop on Structural Materials for Innovative Nuclear Systems, Idaho Falls, ID, October 2013.

Bai, X., "Computational Studies of Oxygen Transport along Grain Boundaries during Zirconium Corrosion," 2013 TMS Annual Meeting, San Antonio, TX, March 2013.

Bai, X., "Computational Studies of Oxygen and Zirconium Diffusion in Tetragonal $\mathrm{ZrO}_{2}$ Related to Zirconium Corrosion," 2012 Computer Simulations of Radiation Effects in Solids, Santa Fe, NM, June 2012. 


\title{
12-056 - Recovery of Precious Metals from Used Nuclear Fuel and Electronic Waste Using Sulfur-Impregnated Nanoscaffolds (SINs)
}

\author{
Peter Zalupski and Linda Nazar ${ }^{1}$
}

Ordered mesoporous carbons (CMKs) are nanostructured carbon materials characterized by a hexagonal, porous molecular framework. The most attractive property of such materials is their porosity of mesoscale dimensions. The diameters of typical pores of mesoporous materials range between 2 and 50 nanometers. As such, a

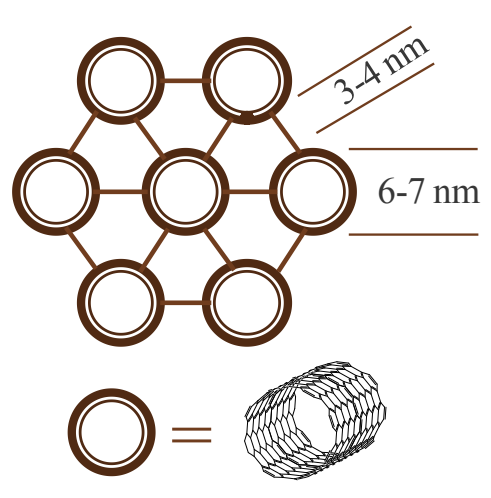
mesoporous framework may be envisioned as the architecture constructed from nano-sized building blocks. CMKs are engineered agglomerates of carbon nanotubes held together by small carbon nanofibers. They exhibit a uniform pore diameter, high pore volume, and high channel permeability. The schematic drawing on the left illustrates a simplified cross-sectional representation of possible architecture, where each circle corresponds to one carbon nanotube. The depth dimension represents the length of the mesoporous structure, which commonly measures several micrometers. Such a mesoporous assembly may span $\sim 100$ channels (i.e., carbon nanotubes of 6-7 nanometer diameters) interlocked into hexagonal unit cells by carbon nanofibers to create wide 3-4 nanometer channel voids. Such porous agglomerates, when coated with molecular sulfur (S), exhibit an attractive affinity for precious metals such as platinum (Pt), gold $(\mathrm{Au})$, and palladium $(\mathrm{Pd})$. This LDRD project evaluates the use of the new nanosized materials, CMKs, in the hydrometallurgical recovery of precious metals from aqueous acidic waste streams.

Research investigations during the initial two years (FY 2012-2013) focused on the fundamental characterization of precious metals adsorption on CMK nanostructures. We have identified a tremendous adsorption capacity of $\mathrm{Au}$ and a less pronounced adsorption of $\mathrm{Pd}$ and $\mathrm{Pt}$. The presence of an elemental $\mathrm{S}$ layer inside the mesoporous framework was illustrated to significantly enhance the uptake of Au but only when suspended in a mildly acidic $(\mathrm{pH} 3)$ chloride-based aqueous environment. At moderately acidic conditions (1 M HCl), the adsorption of a $\mathrm{AuCl}_{4}{ }^{-}$ anion to carbonaceous surfaces competes with a much higher concentration of the chloride anion. Due to this competitive equilibrium, the benefit of using S-impregnated CMK vanishes in acidic media. Actual aqua regia leach mixtures of personal computer and cell phone circuit boards were also used in the sorption studies to reveal the extent of precious metal and other competing metals uptake on the CMK materials. Au adsorption was shown to be very efficient at such complex conditions, containing roughly $5 \mathrm{M}$ total acid. The major drawback of the investigated chemistry is the simultaneous adsorption of tin (Sn) as chlorostannate anions.

\footnotetext{
${ }^{1}$ University of Waterloo, Canada
} 


\section{Summary}

Highlight accomplishments for FY 2014 are listed below:

Year 3 of this investigative effort focused on more applied aspects of this chemistry. As numerous literature studies report significant improvements to the state of art in the area of precious metal adsorption of carbon-rich surfaces, a comparative study was conducted with the goal to collect the adsorption efficiencies of $\mathrm{Au}$ (III) and $\mathrm{Pd}(\mathrm{II})$ for a spectrum of carbon-based porous materials. The investigation indicated which adsorbent is most efficient when equilibrated with a harsh acidic environment of aqua regia leacheates in three types of circuit boards: cell phones, personal computers (PCs), and television (TV) output cards, the compositions of which are listed in Table 1.

Table 1. Composition of aqua regia mixtures after e-scrap leaching process. Total mass (mg) indicates the amount of metal dissolved in a known volume of leach solution (64 mL for cell phone, $416 \mathrm{~mL}$ for PC, $471 \mathrm{~mL}$ for TV). Mass basis composition (ppm or \%) represents metal's mass fraction in studied e-scrap.

\begin{tabular}{|c|c|c|c|c|c|c|}
\hline & \multicolumn{2}{|c|}{ cell phone } & \multicolumn{2}{|c|}{ PC board } & \multicolumn{2}{|c|}{ TV board } \\
\hline & mg in leach & ppm or $\%^{a}$ & $\mathrm{mg}$ in leach & ppm or $\%$ & $\mathrm{mg}$ in leach & ppm or $\%$ \\
\hline $\mathrm{Au}$ & $10.3 \pm 0.7$ & $325 \pm 23$ & $80 \pm 2$ & $386 \pm 8$ & $1.5 \pm 0.4$ & $6 \pm 2$ \\
\hline $\mathrm{Pd}$ & $1.4 \pm 0.1$ & $43 \pm 3$ & $22 \pm 1$ & $106 \pm 4$ & $0.5 \pm 0.2$ & $2.1 \pm 0.8$ \\
\hline $\mathrm{Ag}$ & $2.6 \pm 0.5$ & $82 \pm 16$ & $81 \pm 10$ & $391 \pm 48$ & $29 \pm 1$ & $124 \pm 6$ \\
\hline $\mathrm{Pt}$ & $1.1 \pm 0.1$ & $35 \pm 3$ & $0.56 \pm 0.01$ & $2.7 \pm 0.1$ & - & 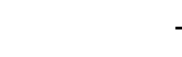 \\
\hline $\mathrm{Hg}$ & - & - & $5.2 \pm 1.5$ & $25 \pm 7$ & $0.04 \pm 0.01$ & $0.19 \pm 0.03$ \\
\hline $\mathrm{Fe}$ & $4570 \pm 194$ & $14.4 \pm 0.6$ & $8032 \pm 356$ & $3.9 \pm 0.2$ & $51482 \pm 2042$ & $21.9 \pm 0.9$ \\
\hline Sn & $922 \pm 20$ & $2.9 \pm 0.1$ & $8498 \pm 47$ & $4.08 \pm 0.02$ & $6613 \pm 242$ & $2.8 \pm 0.1$ \\
\hline Al & $98 \pm 12$ & $0.31 \pm 0.04$ & $4327 \pm 261$ & $2.1 \pm 0.1$ & $2464 \pm 84$ & $1.05 \pm 0.04$ \\
\hline $\mathrm{Cu}$ & $4169 \pm 415$ & $13.1 \pm 1.3$ & $29299 \pm 1765$ & $14.1 \pm 0.8$ & $3526 \pm 414$ & $1.5 \pm 0.2$ \\
\hline $\mathrm{Ni}$ & $420 \pm 25$ & $1.3 \pm 0.1$ & $2426 \pm 61$ & $1.20 \pm 0.03$ & $82 \pm 2$ & $0.04 \pm 0.01$ \\
\hline $\mathrm{Zn}$ & $615 \pm 31$ & $1.9 \pm 0.1$ & $795 \pm 133$ & $0.4 \pm 0.1$ & $9033 \pm 175$ & $3.8 \pm 0.1$ \\
\hline $\mathrm{Pb}$ & $1548 \pm 50$ & $0.74 \pm 0.02$ & $55 \pm 5$ & $0.17 \pm 0.02$ & $1.4 \pm 0.1$ & $<0.01$ \\
\hline
\end{tabular}

a ppm for $\mathrm{Au}, \mathrm{Pd}, \mathrm{Ag}, \mathrm{Pt}, \mathrm{Hg}$

$\%$ for $\mathrm{Fe}, \mathrm{Sn}, \mathrm{Al}, \mathrm{Cu}, \mathrm{Ni}, \mathrm{Zn}, \mathrm{Pb}$

Five carbonaceous platforms were compared: (1) CMK, (2) activated carbon, (3) N-aminoguanidine modified grape skin tannin gel, (4) cross-linked cellulose gel, and (5) CMK impregnated with 2-mercaptobenzothiazole (MBT).

Table 1 indicates the tremendous complexity of the aqueous cocktails resulting from dissolution of electronic scrap in aqua regia. The adsorption of metals from such mixtures was investigated after an initial dilution (9:25) was performed to reduce the concentration of chlorine $\left(\mathrm{Cl}^{-}\right)$anions.

Table 2 summarizes the adsorption efficiencies for metals showing significant uptake (\% uptake > $3 \sigma$ ) from three studied e-scrap leach mixtures on five adsorbent materials. Adsorption of $\mathrm{Au}, \mathrm{Pd}$, and $\mathrm{Sn}$ are observed for all investigated adsorbents. Also, significant adsorption (6-11\%) of iron (Fe) from TV scrap leacheates (presumably 
due to its very high content) was observed on all adsorbent materials. The adsorption of $A u(I I I)$ is nearly quantitative from each type of leach environment for mesoporous (CMK-3) and microporous (charcoal) carbons. In contrast, the efficiency of Au adsorption on biopolymer-based sorbent varies on the composition of leach solutions.

Table 2. Au, Pd and Sn adsorption efficiencies from aqua regia leached cell phone, $P C$, and TV board scrap on CMK, activated charcoal, tannin gel, cellulose gel, and CMK impregnated with MBT.

\begin{tabular}{|c|c|c|c|c|c|c|c|c|c|}
\hline \multirow[b]{2}{*}{ adsorbent } & \multicolumn{3}{|c|}{ Cell phone } & \multicolumn{3}{|c|}{ PC board } & \multicolumn{3}{|c|}{ TV board } \\
\hline & $\mathrm{Au}(\mathrm{III})$ & $\operatorname{Pd}(I I)$ & Sn(IV) & $\mathrm{Au}(\mathrm{III})$ & $\operatorname{Pd}(I I)$ & $\mathrm{Sn}(\mathrm{IV})$ & $\mathrm{Au}(\mathrm{III})$ & $\operatorname{Pd}(I I)$ & Sn(IV) \\
\hline CMK-3 & $99 \pm 5$ & $25 \pm 1$ & $3.8 \pm 0.1$ & $99 \pm 11$ & $8 \pm 1$ & $0.9 \pm 0.1$ & $96 \pm 2$ & $29 \pm 4$ & $6.2 \pm 0.2$ \\
\hline charcoal & $99 \pm 7$ & $35 \pm 1$ & $3.0 \pm 0.1$ & $99 \pm 7$ & $17 \pm 1$ & $3.3 \pm 0.1$ & $99 \pm 2$ & $29 \pm 2$ & $6.1 \pm 0.1$ \\
\hline tannin & $73 \pm 14$ & $36 \pm 1$ & $4.5 \pm 0.1$ & $30 \pm 1$ & $37 \pm 2$ & $1.0 \pm 0.1$ & $16 \pm 1$ & $6 \pm 1$ & $3.4 \pm 0.1$ \\
\hline cellulose & $99 \pm 7$ & - & $1.6 \pm 0.1$ & $18 \pm 1$ & $32 \pm 3$ & $3.1 \pm 0.1$ & $4 \pm 1$ & $9 \pm 1$ & $3.0 \pm 0.1$ \\
\hline CMK/MBT & $99 \pm 12$ & $92 \pm 3$ & $4.0 \pm 0.1$ & $96 \pm 4$ & $80 \pm 3$ & $2.4 \pm 0.1$ & $98 \pm 2$ & $72 \pm 3$ & $4.0 \pm 0.1$ \\
\hline
\end{tabular}

The overall conclusions of this applied comparison of adsorbent materials must focus on the very similar uptake performance observed for mesoporous and microporous carbons. The adsorption properties for $\mathrm{Au}(\mathrm{III}), \mathrm{Pd}(\mathrm{II})$, $\mathrm{Sn}(\mathrm{IV})$, and $\mathrm{Fe}(\mathrm{III})$ are statistically the same for both carbon materials, and only the additional soft-bonding functionalization (CMK/MBT) enhances the Pd adsorption features of such materials. Nonetheless, the utilization of carbonaceous materials to separate precious metals dissolved in aqueous acidic leacheates of electronic scrap has to be accompanied by a follow-on differentiation from Sn and (depending on the type of e-scrap) Fe.

This project supported the internship of Guy Dutech—a French visiting scientist—and the graduate studies of Kaitlin Town from University of Waterloo, Canada.

\section{Benefits to DOE}

The special section of the August 10, 2012 issue of Science has been devoted to the challenges associated with growing production of consumer byproducts. Entitled "Working with Waste," the series of articles highlight the importance of focusing on the recovery of valuable materials. This LDRD project cultivates the belief that our waste may indeed be called treasure instead of trash. Through the sponsorship of this project, DOE is participating in this growing global movement, which, if sustained, will ease the challenges of supporting our society for the years to come.

\section{Publications}

Zalupski, P., R. McDowell, and G. Dutech, "The Adsorption of Gold, Palladium and Platinum from Acidic Chloride Solutions on Mesoporous Carbons," Solvent Extr. Ion Exch., 2014, 32, 737-748.

Zalupski, P., and R. McDowell, "Gold and Palladium Adsorption from Leached Electronic Scrap Using Ordered Mesoporous Carbon Nanoscaffolds," Proceedings of the International Solvent Extraction Conference 2014, Wurzburg, Germany, September 7-11, 2014. 


\title{
12-059 - Chemistry and Flowsheet Development for Lanthanide Separations
}

\author{
R. Scott Herbst, Peter Zalupski, Mitchell Greenhalgh, and Mikael Nilsson ${ }^{1}$
}

The lanthanide series consists of the 15 elements with atomic numbers 57 through 71 (lanthanum [La] through lutetium [Lu]), all occurring naturally with the exception of promethium (Pm). The term "rare earth elements" (REEs) is often used interchangeably with "lanthanides" but also includes the chemically similar elements yttrium $(\mathrm{Y})$ and scandium (Sc). The exceptionally similar chemical characteristics of the REEs are attributed to their existence in the very stable trivalent $(+3)$ oxidation state and the notable similarity in their atomic radii (size). These similar chemical characteristics result in great difficulties during the chemical separation and concomitant purification of these elements. The REE industry currently utilizes chloride media $(\mathrm{HCl})$ for the separation and purification of the REE species. Existing expertise in lanthanide chemistry and nuclear fuels processing will be leveraged to develop and test efficient solvent extraction flowsheets for the separation, purification, and concentration of the individual lanthanides and $\mathrm{Y}$ from each other. The emphasis will focus on solvent extraction processing from nitrate media (nitric acid). Changing the extraction media to $\mathrm{HNO}_{3}$ would make the system compatible with concepts and equipment standard to reprocessing used nuclear fuels and allow extraction systems to be studied in an existing 30 stage centrifugal contactor pilot plant. This departure from the norm (i.e., chloride- or $\mathrm{HCl}$-based extraction systems in classical mixer-settlers) opens numerous possibilities, including the use of non-equilibrium or reaction kinetics to define more efficient separations. The end goal is to develop process models and design flowsheets that will significantly improve process economics by utilizing one or more of the following principles: increased throughputs, improved separation factors, reduced chemical costs/inventories, reduced waste costs, or reduced conversion costs for products.

\section{Summary}

During the first year of this project, an initial investment was made in procuring many of the REEs in the form of high purity $(99+\%)$ nitrate salts for use as feed stocks for the separation experiments. Additionally, samples of radioactive isotopes of cerium $(\mathrm{Ce}), \mathrm{Y}$, gadolinium $(\mathrm{Gd})$, and holmium $(\mathrm{Ho})$ were obtained for determination of distribution ratios in a variety of solvent extraction tests. These materials provide a convenient analytical means to determine extraction behavior of individual elements from a mixture of REEs; Ce, Y, Gd, and Ho can be analyzed simultaneously and directly from solutions containing mixtures of the four elements. The radiometric analysis method is also very accurate and sensitive and provides quick analytical turnaround for scoping tests.

Additionally, procurement, setup, and testing of a new Mettler-Toledo titration system were completed during the first year. This analytical equipment provides a rapid means to evaluate REE concentrations in aqueous solutions and can be used to evaluate single element concentrations of the REE by colorimetric complexation titration using a phototrode. This method has also proven valuable for the rapid evaluation of REE behavior in experimental scoping studies and in a non-radiological environment.

REE separation evaluations were completed during the second year of this project on multiple rare earth extractants in nitrate media. The laboratory scale evaluations were performed on three organophosphorous extractants (Cyanex-923, Cyanex-272, and Cyanex-572) supplied by Cytec Industries, on di (2-ethyl hexyl) phosphoric, and on 2-ethylhexyl phosphonic acid mono-2-ethylhexyl ester (PC88A). Each of these extractants

\footnotetext{
${ }^{1}$ University of California, Irvine
} 
was evaluated for acid dependency, diluent effects, synergistic effects, metal loading, optimal stripping conditions, and separation of the individual REEs from each other. No synergistic effects were observed with any of the extractants tested. Cyanex-923 in an Isopar-L diluent was found to have the highest extraction efficiency for the lanthanides at a nitric acid concentration of $0.1 \mathrm{M}$. However, separation between the individual elements tested was low. Cyanex-572 in an Exxsol D80 diluent showed lower extraction efficiencies than the Cyanex-923, but it demonstrated a much higher selectivity for the heavier rare earths (i.e., Ho and erbium [Er]) and Y. Cyanex-272 resulted in the lowest extraction efficiencies and is no longer being considered as an option. The PC88A extractant showed the highest separations between the REEs and, based on these results, was chosen as the extractant to evaluate in a full flowsheet test in nitrate media. The data obtained from the PC88A laboratory tests were utilized to build a process model and to develop a flowsheet for testing in the 30 stage centrifugal contactor pilot plant.

In the final year of the project, a process model was developed from the data obtained with the PC88A extractant and a flowsheet was designed from the modeling results. Flowrates, organic to aqueous ratios, and acid concentrations were all defined for the extraction, scrub, and strip sections of the flowsheet. The 30 stage pilot plant was configured for the flowsheet as defined, and a test was performed with the pilot plant while guests Mikael Nilsson and Tro Babikian from the University of California, Irvine attended as observers. Samples of both the organic and aqueous phases were taken throughout the test duration to monitor the approach to the steady state of both equilibrium acid concentration and the metals concentration. The equilibrium metal concentrations were also utilized to determine the separation efficiencies between the metals. The results of the test demonstrated a separation of $\mathrm{Gd}$ (i.e., the mids) from $\mathrm{Er}$ and $\mathrm{Y}$ (i.e., the heavies) under the conditions tested, which validated the model's predictions.

\section{Benefits to DOE}

The success of this work is important to ensure an economical supply of lanthanide elements (identified as critical and strategic materials) for a multitude of high-tech industries and applications for certain DOE clean energy technologies and national security arenas. The data and knowledge generated from this LDRD project have the potential to impact the economics and supply of these "critical materials" in a favorable manner and most certainly will be applied to future research as part of the Critical Materials Institute's efforts. 


\title{
12-077 - Observation of Zirconium Oxidation at Atomic Level Using Non-Linear Optical Spectroscopy
}

\author{
Marat Khafizov, Kenan Gundogdu, ${ }^{1}$ and Izabela Szlufarska ${ }^{2}$
}

Understanding of the atomic level mechanisms underlying the initial oxidation kinetics of zirconium $(\mathrm{Zr})$ metal will benefit further development of $\mathrm{Zr}$ alloys as a cladding material in nuclear fuels, especially in the development of accident tolerant nuclear fuel. We seek an integrated approach that brings together advanced spectroscopic techniques and state-of-the-art first principle calculations. We implement Second Harmonic Generation (SHG) spectroscopy, a nonlinear optics technique that provides bond specific information about chemical and structural properties of interfaces. We use this technique to study the formation of an initial oxide layer on the surface of single crystal Zr metal. In conjunction with the SHG experiments, we perform ab initio calculations based on the density functional theory. These calculations are highly accurate and particularly beneficial in discovering preferential bonding of oxygen $(\mathrm{O})$ in $\mathrm{Zr}$ alloys as a function of applied strain. In addition, advanced, time dependent density functional theory methods are used to quantify hyperpolarizabilities of $\mathrm{Zr}-\mathrm{O}$ bonds. The results of those calculations are applied to interpret the SHG spectra.

\section{Summary}

On the simulation side, we have been focusing on understanding the nucleation and growth of $\mathrm{Zr}$ suboxides at the advancing metal/oxide interface. The $\mathrm{Zr}-\mathrm{O}$ system is comprised of a variety of ordered $\mathrm{ZrO}_{x}$ phases obtained by $\mathrm{O}$ atoms occupying the octahedral sites in hexagonal close-packed $\mathrm{Zr}$. Previous ab initio calculations published in the article "Thermodynamics of the $\mathrm{Zr}-\mathrm{O}$ system from first-principles calculations" by B. Puchala and

A. Van der Ven established the presence of three major ground states of the suboxides, $\mathrm{ZrO}_{1 / 6}, \mathrm{ZrO}_{1 / 3}$, and $\mathrm{ZrO}_{1 / 2}$. Experiments using transmission electron microscopy ("Transmission Electron Microscopy Examination of Oxide Layers Formed on Zr Alloys" by A. Yilmazbayhan, E. Breval, A.T. Motta, and R.J. Comstock and "How the Crystallography and Nanoscale Chemistry of the Metal/Oxide Interface Develops during the Aqueous Oxidation of Zirconium Cladding Alloys" by N.Ni, D. Hudson, et al.) identified the formation of $\mathrm{ZrO}_{1 / 3}$ near the surface. The correlation between the suboxide phase and an improved corrosion resistance of $\mathrm{Zr}$ alloys was also reported by A.T. Motta, A. Yilmazbayhan, M.J.G. da Silva, R.J. Comstock, G.S. Was, J.T. Busby, E. Gartner, Q. Peng, Y.H. Jeong, and J.Y. Park in "Zirconium Alloys for Supercritical Water Reactor Applications: Challenges and Possibilities." Therefore, it is of great technological interest to understand why only $\mathrm{ZrO}_{1 / 3}$ precipitates are observed in transmission electron microscopy experiments and, more importantly, how these suboxide phases grow and are distributed at the metal/oxide interface.

To answer these questions, we proposed the method that combines both cluster expansion and cluster dynamics (CD). To exercise cluster expansion, B. Puchala and A. Van der Ven fit effective cluster interactions between pair clusters, triplets, and quadruplets based on density functional theory energies and then formed the system Hamiltonian. We applied the Hamiltonian to calculate the interfacial energy between $\mathrm{Zr}$ and different suboxides and the formation energies of $\mathrm{O}$ interstitial as a function of $\mathrm{O}$ concentration. It was found that the interfacial energies fell into the normal energy range of cohesive interfaces $\left(20 \mathrm{~mJ} / \mathrm{m}^{2}\right.$ or less).

\footnotetext{
${ }^{1}$ North Carolina State University

${ }^{2}$ University of Wisconsin-Madison
} 
The formation energy and interfacial energy were then used as input parameters for CD calculations. In "Cluster Dynamics Modelling of the Precipitation Kinetics in Al(Zr,Sc) Alloys," P. Guyot and C. Sigli define CD as a numerical method that solves differential equations expressing the balance of solute exchanges between clusters (or precipitates) for each time increment. The computation of the precipitate size distribution would give a complete description of the precipitates kinetic evolution. For understanding the suboxide nucleation in $\mathrm{Zr}$, we designed the following two-step simulation scheme. In Step I, we will develop a "one solute-three phases" $\mathrm{CD}$ code. Here "one solute" refers to an O monomer (or interstitial) and "three phases" refers to $\mathrm{ZrO}_{1 / 6}, \mathrm{ZrO}_{1 / 3}$, and $\mathrm{ZrO}_{1 / 2}$. Based on this code, we will be able to predict the precipitation kinetics at a certain temperature and $\mathrm{O}$ concentration, as well as the final precipitate phase. At present we have completed the code for "one solutionone phase." For example, Figure 1 shows results of $\mathrm{ZrO}_{1 / 3}$ nucleation from an $\mathrm{O}$ solution with a fixed concentration of 25 at. $\%$ and at $773 \mathrm{~K}$. As $\mathrm{ZrO}_{1 / 3}$ precipitates out, the $\mathrm{O}$ monomer concentration decreases (Figure 1a) and the mean cluster radius of $\mathrm{ZrO}_{1 / 3}$ becomes larger (Figure 1b). The cluster density increases initially but then decreases because small $\mathrm{ZrO}_{1 / 3}$ clusters merge into larger ones (Figure 1c). In Step II, once the "one solute-three phases" code is finished, we will connect the nucleation process with $O$ diffusion by solving coupled CD codes using parallel computing. As shown in Figure 2, the O-rich region between $\mathrm{Zr}$ oxide and pure $\mathrm{Zr}$ metal is segmented into several suboxide layers. The $\mathrm{O}$ concentration in each layer is set initially to be a function of its depth, but then will be updated according to the $O$ diffusion between layers and $O$ consumption due to nucleation in each layer. This step would generate the distribution of suboxide phases along the $\mathrm{O}$ concentration profile from $66.7 \%$ at $\mathrm{m}-\mathrm{ZrO} 2$ to $0 \%$ in pure $\mathrm{Zr}$ bulk.
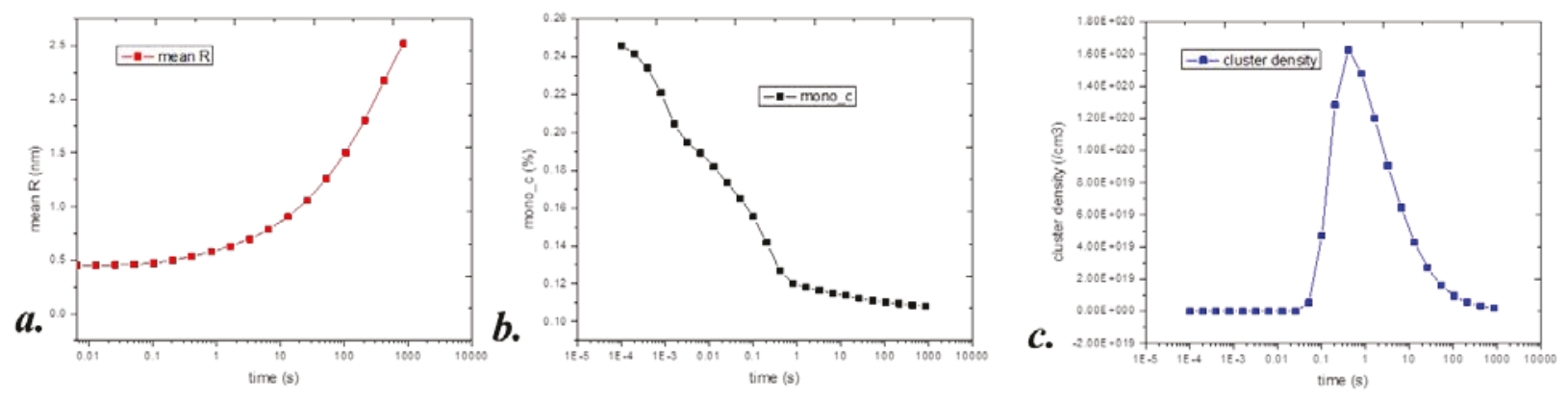

Figure 1. $C D$ results on nucleation of $\mathrm{ZrO}_{1 / 3}$ precipitate from 25 at. $\% \mathrm{Zr}$-O solutions at $773 \mathrm{~K}$ : (a) $\mathrm{O}$ monomer concentration vs. time, (b) average Zr3O cluster size vs. time, and (c) Zr3O cluster density vs. time.

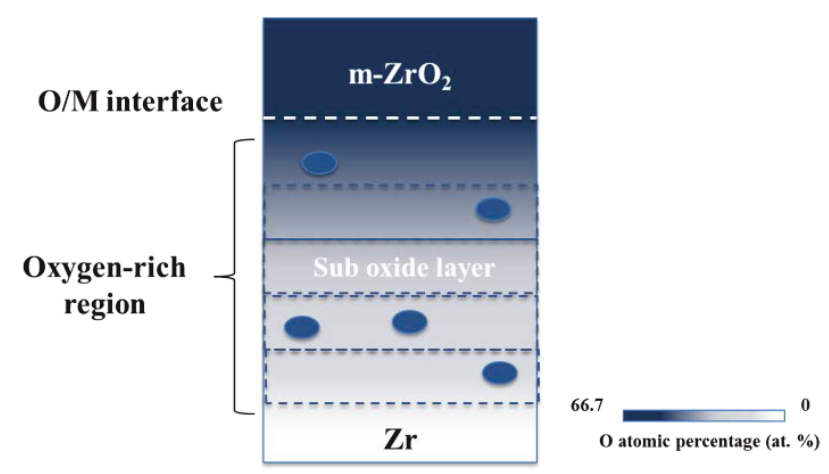

Figure 2. O concentration profile between Zr oxide and pure Zr metal. Suboxide layers are marked with dashed lines. 
On the experimental side, a recipe to achieve a fairly smooth interface in $\mathrm{Zr}(0001) / \mathrm{ZrO}_{2}$ has been explored using chemical etching and fine polishing in ambient conditions. We have used nonlinear optical rotational anisotropy to show that the interface is relatively smooth; this is a preparation done before introducing the sample into the ultrahigh vacuum chamber. Within the ultrahigh vacuum chamber, a fresh $\operatorname{Zr}(0001)$ surface has been processed, and oxidation upon $\mathrm{O}_{2}$ exposure has been studied using SHG and Auger spectroscopy (Figure 3). It has been found that the initial oxidation and/or adsorption on $\operatorname{Zr}(0001)$ has a time scale of $\sim 0.42$ Langmuir, which has not been reported before, to the best of our knowledge. Typically, electrons in metal are free, thus the surface of fresh $\mathrm{Zr}$ gives a higher SHG signal. When oxides are formed on $\mathrm{Zr}$, free electrons are bonded into covalent bonds, reducing the SHG signal tremendously. The time scale of $\sim 0.42$ Langmuir shows the trapping of $O$ onto the surface of $Z r$ is very fast, a sign of high reactivity between $\mathrm{Zr}$ and $\mathrm{O}$.
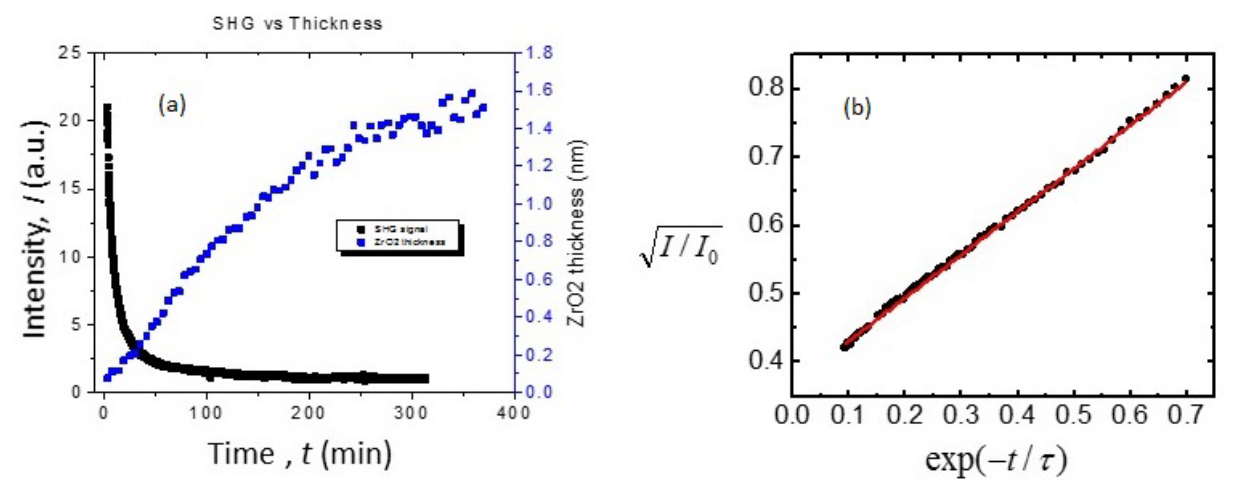

Figure 3. (a) Oxidation kinetics revealed by SHG and Auger spectroscopy and (b) demonstration of Langmuir kinetics.

\section{Benefits to DOE}

DOE has interest in the development of alloys and coatings that have improved corrosion characteristics. Methods developed under this program can be implemented to guide development of materials with desired chemical and physical properties. The Consortium for Advanced Simulation of Light Water Reactors is developing a macro-scale model of oxidation of $Z r$ to determine the overall rate of oxide growth, but the nucleation of suboxide was not considered. Our modeling of suboxide formation ahead of the oxidation front is complementary to that model and could be possibly incorporated in the future. First principle calculations done by M.V. Glazoff in "Oxidation and Hydrogen Uptake in Zirconium, Zircaloy-2 and Zircaloy-4: Computational Thermodynamics and Ab Initio Calculations" showed that a $\mathrm{ZrO}_{1 / 3}$ precipitate slows down the hydrogen $(\mathrm{H})$ diffusion inside of the bulk $\mathrm{Zr}$ alloys, suggesting that the $\mathrm{ZrO}_{1 / 3}$ may be very important to suppress the $\mathrm{H}$ take-up. Understanding the nucleation of $\mathrm{Zr}$ suboxide would help predict the $\mathrm{H}$ pick-up in $\mathrm{Zr}$ alloys and benefit DOE's used fuel disposition mission/ 


\section{Publications}

Wang, X., M. Khafizov, and I. Szlufarska, "Effect of Surface Strain on Oxygen Adsorption on Zr(0001) Surface," J. Nucl. Mater., Vol. 445, 1-6, 2014.

"Nucleation of Zr suboxide phases near the metal/oxide interfaces," in preparation.

\section{Presentations}

Wang, X., and I. Szlufarska, "Effects on Surface Strain on Oxygen Adsorption on Zr(0001) Surface," The Minerals, Metals, and Materials Society (TMS) Annual Meeting, San Antonio, TX, March 3-7, 2013. 


\title{
13-095 - Development and Validation of a Societal-Risk Goal for Nuclear Power Plant Safety
}

\author{
Robert Youngblood, Michael Corradini, ${ }^{1}$ and Vicki Bier ${ }^{1}$
}

Existing safety goals for commercial nuclear power plants are focused on consequences to individuals rather than consequences to society and concentrate explicitly on radiological dose as opposed to the adverse effects of relocation for indefinite periods. This goal structure does not fully reflect the range of reactor accident consequences that need to be avoided. This project is evaluating the social disruption effects from severe reactor accidents as a basis for developing a societal-risk goal for nuclear power plants. The work is considering both health effects and non-health concerns like property damage and land interdiction. To achieve our goal, we are generating and using data on accident consequences at various reactor sites in the U.S. to assess the implications of concern about societal risk for nuclear-power regulation.

The project began by studying the possible societal disruptions that could occur from nuclear-power accidents, considering both past accidents and potential future accident scenarios. One important finding is the observation that an improved societal-risk goal for nuclear-power plants should ideally account for not only anticipated health effects but also the number of people that would need to be evacuated in the event of an accident. This is an important addition to traditional safety-goal policies for two reasons. First, the number of people to be evacuated appears to be a good proxy for the level of societal disruption from an accident while still being relatively straightforward to compute in an objective manner. Second, health effects and evacuation trade off directly against each other. For example, even a large accident could have negligible health effects if the number of people evacuated was sufficiently large, but that does not mean it would cause negligible societal disruption. Thus, the ultimate purpose of the project is to enhance the traditional Nuclear Regulatory Commission safety-goal policy through development of a societal-risk goal that places a limit on the aggregate extent of disruption that could result from a severe accident.

\section{Summary}

We were able to quantify the offsite impacts from severe reactor accidents at five plants (actually six, but we left out San Onofre Nuclear Generating Station because it is ceasing power generation) on 24 different dates over a period of 2 years (2011 and 2012) using actual site-specific weather data. The plants chosen include high- and low-population sites. We used the number of people relocated as a proxy for societal disruption. Using RASCAL, we were able to produce these numbers for time frames of 4 days, 1 year, 2 years, and 50 years after the accident and at various different thresholds for relocation ( 0.5 Rem through 4 Rem). Ultimately, we chose to focus on the results 1 year after an accident as being representative of the greatest societal disruption. (Being relocated for only 4 days is not too disruptive if people are able to move back soon afterwards, while if relocation continues much longer than 1 year, many people will have settled into a "new normal" and no longer experience ongoing disruption.)

We studied the effects of different protective-action thresholds for relocation. Lower thresholds result in fewer cancer fatalities, but relocation of more people, while higher thresholds result in fewer people relocated at the expense of more cancer fatalities. We were able to quantify the tradeoff between cancer fatalities and relocation

\footnotetext{
${ }^{1}$ University of Wisconsin-Madison
} 
(i.e., roughly how many people would need to be relocated to prevent one cancer fatality). Although results vary somewhat by location and accident scenario, to an order of magnitude, roughly 1000 people would need to be relocated for each cancer fatality prevented. (Note also that our results may overestimate the number of cancers resulting from an accident due to the use of the linear no-threshold dose-response model.) We have currently begun evaluating different forms for quantifying the combined impact of cancer fatalities and relocation. Our sensitivity analysis includes parameters such as how much weight to give to fatalities versus relocation, whether to incorporate risk aversion with respect to large numbers of people relocated (via an exponent slightly greater than one on the relocation numbers), and whether there are interaction effects (e.g., whether avoiding large numbers of cancer fatalities is considered more important following a large relocation than following a small relocation).

One student participating in the project has completed his Ph.D. and is currently on faculty at the Air Force Institute of Technology. He is continuing to collaborate with the project. We have also collaborated with a number of individuals active in emergency planning at the Nuclear Regulatory Commission and participated in a workshop on assessment of the risk of extreme floods to pursue commonalities in the assessment and management of severe accidents.

\section{Benefits to DOE}

The work has already generated insights into possible improvements in evacuation policy for large accidents (e.g., by extending emergency-planning zones beyond 10 miles if needed). Similar methods (using state-of-the-art dispersion models and geographic-information systems) should be applicable to emergency planning in other areas as well (chemical emergencies, military planning, etc.). The results of this work should help to build public support for appropriate deployment of new nuclear plants in the aftermath of the events at Fukushima. Better modeling of societal disruption (rather than relying on relatively crude proxies, such as number of fatalities) is a particularly novel aspect of the work.

\section{Publications}

Bier, V., M. Corradini, R. Youngblood, C. Roh, and S. Liu, "Candidate Societal-Risk Goals for Nuclear Power Safety," to be submitted September 2015.

\section{Presentations}

Bier, V., M. Corradini, R. Youngblood, C. Roh, and S, Liu, "Development of an Updated Societal-Risk Goal for Nuclear Power Safety," Probabilistic Safety Assessment and Management, Hawaii, June 2014. 


\title{
13-105 - Micro/Nano Scale AFM-based Thermal Conductivity Measurement and Atomistic Modeling for Oxide Fuel: The Effects of Grain Boundary, Fission Gas and Radiation Damage
}

\author{
Jian Gan, Xianming "David” Bai, Yong Yang, ${ }^{1}$ Heng Ban, ${ }^{2}$ and Aleksandr Chernatynskiy ${ }^{1}$
}

Although considerable progress has been made toward modeling the thermal conductivity degradation as a function of temperature, burn up rate, and fuel chemistry, there is lack of fundamental understanding on the thermal resistance from each individual type of micro/nano-structural and chemical features that form during fabrication and evolve under irradiation.

This research effort studies the microstructure and radiation effects on the thermal conductivity of oxide fuel using a novel Atomic Force Microscopy based measurement technique and complementary computer modeling. By using a bottom-up approach, the effects of tailored microstructures and controlled radiation-damage on materials' thermal conductivity can be individually evaluated and modeled. The complementary atomistic modeling of the thermal transport is conducted to compare the results directly with experiments. Specifically, the following objectives are to be accomplished: (1) determine grain boundary (GB) thermal resistance for different types of GBs in undamaged materials to investigate how specific GB structure affects the thermal transport; (2) investigate the thermal resistance of GBs with fission gas implantation by considering that different GBs may have different effects on fission gas segregation; (3) determine thermal conductivity degradation caused by radiation damage at different irradiation temperatures.

\section{Summary}

\section{Modeling Effort}

Comprehensive investigation of the Kapitza resistance in $\mathrm{CeO}_{2}$ as a function of $\mathrm{GB}$ type (twist or tilt) orientation (001, 011, or 111) and misorientation angles was successfully conducted and analyzed with the help of the extended Read-Shockley model. This resulted in the simple expression of the GB Kapitza resistance as a function of the misorientation angle. A publication presenting the results of this effort is currently in preparation.

Studies of the effect of defect segregation at GBs on thermal transport are in progress in two directions: investigation of the effect of the off-stoichiometry at $\mathrm{GB}$ in $\mathrm{CeO}_{2}$ and development of the $\mathrm{CeO}_{2}$-krypton $(\mathrm{Kr})$ potential. For the former, oxygen $(\mathrm{O})$ vacancies were created at $\mathrm{GBs}$ to study how O-deficit $\mathrm{GBs}$ in $\mathrm{CeO}_{2-\mathrm{x}}$ affect the thermal resistance. The GB thermal resistance increases almost linearly with the local off-stoichiometry. Using an off-stoichiometric layer in a single crystal as a reference, we found that GB structure dominates the thermal resistance at low off-stoichiometry, and $\mathrm{O}$ defects dominate the resistance at a high off-stoichiometry condition. The atomistic results were then input into an analytical model to evaluate the effects of segregation of $O$ vacancies on thermal transport at realistic length scales. We found that the segregation of $O$ vacancies improves the thermal transport, especially when the grain size is less than $400 \mathrm{~nm}$. The results may explain why nanograined, high-burnup structures have improved thermal conductivity once they form. For the latter, the

\footnotetext{
${ }^{1}$ University of Florida

${ }^{2}$ Utah State University
} 
database for the potentials fitting is produced using density functional theory calculations that contain various configurations of $\mathrm{Kr}$ atoms within a $\mathrm{CeO}_{2}$ lattice. The Buckingham form of the potential was identified as the most suitable form for the $\mathrm{Kr}$-cerium $(\mathrm{Ce})$ and $\mathrm{Kr}-\mathrm{O}$ interactions, and the fitting is in progress.

\section{Experimental Effort}

As shown in Figure 1 below, $\mathrm{CeO}_{2}$ pellets with an average grain size larger than $50 \mu \mathrm{m}$ were successfully fabricated using the isostatic pressing and sintering process. The sintering was conducted at $1600^{\circ} \mathrm{C}$ and was followed by a high temperature annealing at $1800^{\circ} \mathrm{C}$ in air for 4 hours. $96 \%$ theoretical density was achieved. The $\mathrm{CeO}_{2}$ samples were subjected to a $\mathrm{Kr}$ implantation at 200 and $600^{\circ} \mathrm{C}$ with the damage doses ranging from 0.3 to $150 \mathrm{dpa}$. The dislocation structure and fission gas bubble morphology are to be studied. In addition, the theoretical modeling was constructed by considering the phonon scattering from point defects, dislocation loops, and GB thermal resistance.

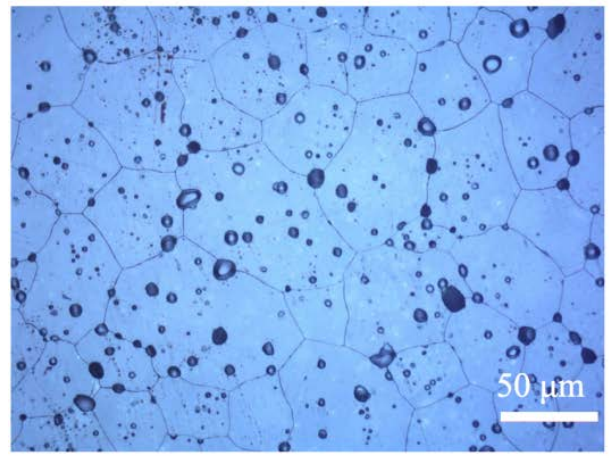

Grain structure of sintered $\mathrm{CeO}_{2}$

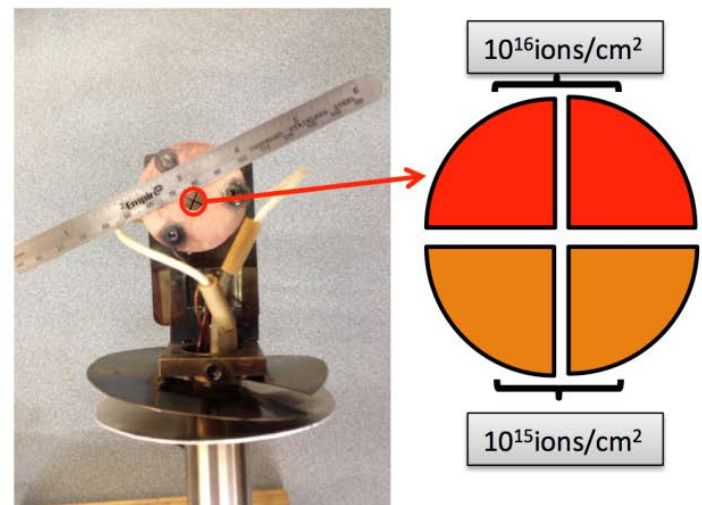

Irradiation sample stage

Figure 1. $\mathrm{CeO}_{2}$ pellets fabricated through the isostatic pressing and sintering process.

Work on thermal resistance measurement has been done on two fronts. First, fabrication is completed for an artificial GB using single crystal silicon and measurement of its thermal resistance. This work is done to generate well-defined GBs for optical measurement and provide samples for testing the measurement methods being developed. The results can be used for calibration of computational predictions. Second, the measurement of the $\mathrm{CeO}_{2}$ sample provided by the University of Florida has commenced. This work is in the beginning process of the initial testing, using lasers directly on the bare sample. A phase change for thermal wave propagation was detected. An analytical model for the semi-transparent sample is being developed for data extraction. All tests performed are non-destructive without a coating in order to use the sample later with a titanium (Ti) coating for the eventual measurement using optical reflectance. Both samples are being further tested in the lab. In addition, Atomic Force Microscopy measurement will be conducted on these samples.

This project supported four graduate and postdoctoral scholars: Colby Jensen started as a graduate student working on his Ph.D. degree at Utah State University and is now working as a postdoctoral researcher on this project; Kurt Harris is a graduate student working on his Ph.D. degree at Utah State University; Zilong Hua is a postdoctoral scholar working on this project at Utah State University; and Yuedong Wu is a graduate student working on his Ph.D. degree at University of Florida. 


\section{Benefits to DOE}

Light Water Reactor parameters, such as nominal power level and fuel temperature margin to failure, have been limited by the poor thermal conductivity of oxide fuel and the resulting large temperature gradient. A better understanding of the thermal conductivity of oxide fuel during operation will have a significant impact on developing advanced light water reactor fuels with improved thermal physical properties, which can enhance the safety and performance of commercial nuclear fuels. The fundamental study will also contribute to DOE's leading role on basic science research of materials' behavior and performance in extreme environments.

\section{Publications}

Zheng, Z., X. Chen, B. Deng, A. Chernatynskiy, S. Yang, L. Xiong, and Y. Chen, "Phonon Thermal Transport through Tilt Grain Boundaries in Strontium Titanate," J. Appl. Phys., Vol. 116, 2014.

\section{Presentations}

Chernatynskiy, A., X. Bai, and J. Gan, "Thermal Boundary Resistance and Irradiation Effects of the Grain Boundaries in Ceria," 2015 TMS Meeting, Orlando, FL, March 15-19, 2015, accepted.

Bai, X., A. Chernatynskiy, and J. Gan, "Effects of Misorientation, Temperature, and Stoichiometry on Grain Boundary Thermal Resistance in $\mathrm{CeO}_{2}, " 2014$ Nuclear Materials Conference (NuMat2014), Clearwater, FL, October, 2014.

Chernatynskiy, A., X. Bai, and J. Gan, "Kapitza Resistance of the Grain Boundaries in Ceria," 2014 ANS Meeting, Reno, NV, June 15-19, 2014, poster.

Bai, X., A. Chernatynskiy, and J. Gan, "Misorientation-Dependence of Grain Boundary Thermal Resistance in $\mathrm{CeO}_{2}$," 2014 TMS Annual Meeting, San Diego, CA, February 2014.

Wu, Y., H. Ban, X. Bai, A. Chernatynskiy, J. Gan, and Y. Yang, "Correlation Between Thermal Conductivity and Microstructural Evolutions in $\mathrm{CeO}_{2}$ Upon Radiation and Fission Gas Implantation," 2014 TMS symposium: Radiation effects in oxide ceramics and novel LWR fuels, San Diego, CA, February 16-20, 2014. 


\title{
13-110 - Nuclear-Renewables-Oil Shale Hybrid Energy System
}

\author{
Charles Forsberg, ${ }^{1}$ Daniel Curtis, ${ }^{1}$ and Humberto Garcia
}

This project addresses four goals:

1. Define the design requirements for a new, large energy system to address the challenges of flexible, dispatchable, very-low-carbon $(\mathrm{C})$ electricity generation and lower-C transportation fuel production

2. Describe the configuration of nuclear renewable oil shale systems (NROSSs) that satisfies those requirements

3. Analyze a selection of performance measures of NROSS, chosen to demonstrate the value and viability of the system concept

4. Describe the necessary R\&D, institutional partnerships, demonstration systems, and commercialization activities (collectively, a "development pathway") necessary to deploy NROSS.

NROSSs are hybrid energy systems that provide base-load nuclear reactors the primary energy used to produce shale oil from kerogen deposits and flexible, dispatchable electricity to the grid. NROSS couples electricity and transportation fuel production in a single operation, reduces lifecycle $\mathrm{C}$ emissions from the fuel produced, improves economics for the nuclear plant, and enables a major shift toward a very-low-C electricity grid. The nuclear reactor driving an NROSS system would operate steadily at full power providing steam for shale heating in closed steam lines when the price of electricity is low and electricity to the grid when the price of electricity is high. Because oil shale has low thermal conductivity, heat input to the shale can be cycled as needed without disrupting the steady increase in average temperature. During Stage I heating, the steam from light water reactors (LWRs) heats the kerogen. This heating is done when electricity prices are low. During Stage II kerogen heating, higher temperature steam is produced by electric heating with electricity bought from the grid at times of low electricity prices. This reduces the electricity price collapse associated with large-scale deployment of wind or solar at times of high wind or solar output. Current analyses show that fuels produced by a baseline NROSS would have the lowest life cycle greenhouse gas (GHG) impact of any presently available fossil fuels and that operation as part of an NROSS complex would increase reactor revenues by $41 \%$. The flexible, dispatchable electricity provided by NROSS could also enable the transition to a very-low-C grid where renewables are widely deployed and the NROSS provides variable output to balance their uncontrolled output to meet demand.

\section{Summary}

Work to date has provided preliminary results on all four major project goals described above. Study of U.S. energy systems has shown that flexible, dispatchable, low-C electricity generation is technically achievable today but at an extremely high cost using purpose-built and optimized load-following LWRs or energy storage systems. NROSS overcomes these limitations through the capability to vary instantaneous heat input to the oil shale without disrupting the slow increase in temperature of the shale. This allows the reactor plant to output exactly the electric power needed by the grid while fully utilizing the reactor thermal power, sending the balance of heat to the shale. Economically, full utilization of reactor thermal power is a necessity for competitive nuclear economics. NROSS therefore satisfies the requirement for a flexible, dispatchable, very-low-C electricity generator with competitive economics. In situ oil shale heating using closed steam lines has been selected as our baseline heat

\footnotetext{
${ }^{1}$ Massachusetts Institute of Technology
} 
delivery subsystem. However, recent developments in near-surface enclosed capsule heating make this a promising alternative that should be watched closely.

NROSS has been shown to have the ability to achieve quantitatively superior nuclear plant economics and reduced GHG emissions in liquid fuel production. The baseline LWR system could achieve a $41 \%$ revenue increase over base-load operation with steam sales to a petroleum system operator managing the shale heating and hydrocarbon recovery and electricity sales to the California Independent System Operator. An alternative operating model, with a single owner receiving revenues for sales of both hydrocarbon products and electricity, showed a revenue increase over base-load operation greater than $90 \%$. GHG emissions of fuels from the first-of-a-kind NROSS operation will be competitive with present crude refining, with large-scale development of a low-C grid, and future process optimizations reducing NROSS emissions below anticipated conventional processes. Particular achievements include:

1. Identified a range of suitable system configuration options

a. Several power options: LWRs of various sizes or advanced high temperature reactors

i. Baseline option is a small modular LWR.

b. Two subsystem options for oil shale heating and processing.

i. Baseline option is closed steam lines in solid oil shale with conventional drilling for hydrocarbon extraction

ii. Near-surface enclosed capsule of mined oil shale offers a very promising alternative currently under development at Red Leaf Resources in Utah and presents a potential collaboration opportunity for INL.

2. Analyzed a subset of system configuration options for revenue performance and GHG emissions

a. Baseline NROSS achieves a $41 \%$ revenue increase for the reactor operator with steam sales to the petroleum system operator and electricity sales to the California market

b. Baseline NROSS is competitive with current crude refinement on lifecycle GHG emissions; low-C grid development and process optimization present major opportunities for GHG emission reduction.

3. Described innovations needed in facility and organization design for optimal deployment of a large array of reactors for primary power.

\section{Benefits to DOE}

NROSS, if successfully developed on a large scale, would (1) have the lowest greenhouse footprint of any method to produce fossil-based liquid fuels, (2) enable a zero-C western electricity grid, (3) assure U.S. supplies of liquid fuels, and (4) provide a large market for nuclear reactors. This system could simultaneously increase energy security in both the electric sector and transportation sector in a single operation. It could serve as a major enabler of low-cost mass production of small modular LWRs by providing a customer for an array of tens or hundreds of them. The large amount of flexible, dispatchable electricity provided could eliminate constraints on development of large-scale intermittent renewable generators in the western half of the country. 


\section{Publications}

Curtis, D., C. Forsberg, M. McKellar, and E. Mattson, "A Demonstration Project Coupling a Small Modular Reactor with a Hybrid Nuclear Renewable Oil Shale System," Nuclear Technology, submitted, September 2014.

\section{Presentations}

Curtis, D., C. Forsberg, M. McKellar, and E. Mattson, "A Demonstration Project Coupling a Small Modular Reactor with a Hybrid Nuclear Renewable Oil Shale System," International Congress on Advances in Nuclear Power Plants (ICAPP), Charlotte, NC, April 6-9, 2014.

Curtis, D., and C. Forsberg, "Nuclear Renewable Oil Shale System (NROSS)—Lowest GHG Impact Liquid Fuels and Flexible Electricity Output," (poster), 2014 ANS Winter Meeting, Anaheim, CA, November 9-13, 2014.

Forsberg, C., and D. Curtis, "Nuclear Renewable Oil Shale System (NROSS)—Making Oil Shale the Fossil Fuel with the Lowest Greenhouse Impact per Liter of Diesel or Gasoline While Improving Economics," 34th Colorado Oil Shale Symposium, Golden, CO, October 13-17, 2014.

Curtis, D., and C. Forsberg, "Nuclear Oil Shale-Partnership Opportunity for an Expanded Domestic SMR Market," 2013 ANS Winter Meeting, Washington, D.C., November 10-14, 2013.

Curtis, D., and C. Forsberg, "Nuclear Heat and Power for In-Situ Shale Oil Production and Variable Electricity," 33rd Colorado Oil Shale Symposium, Golden, CO, October 14-16, 2013.

Curtis, D., and C. Forsberg, "Light Water Reactor Arrays for Production of Shale Oil and Variable Electricity," 2013 ANS Annual Meeting, Atlanta, GA, June 16-20, 2013.

Kadak, A., D. Curtis, and C. Forsberg, "Integrated Nuclear Shale Oil System-Lowest- $\mathrm{CO}_{2}$-Emission Fossil Liquid Fuels and Variable Electricity," (poster), International Oil Shale Symposium 2013, Talinn, Estonia, June 10-13, 2013.

Curtis, D., and C. Forsberg, "Nuclear Shale Oil Development-Reactor Arrays and System Effects," (poster), 2013 ANS Student Conference, Boston, MA, April 4-6, 2013. 


\section{CENTER FOR ADVANC ENERgr STUDIES}




\title{
12-086 - Alert/Alarm Dashboard
}

\author{
Ronald L. Boring, David I. Gertman, and Brian F. Dyre ${ }^{1}$
}

While full-scale, high-fidelity control room simulators exist for research on displays and control systems and training of operators, their utility is limited by high demand on the technical staff and expensive facilities that greatly increase overhead costs. Research with such simulators also requires a pool of trained operators-whose time is very constrained-to act as research participants. These factors are significant barriers to conducting efficient empirical research on novel display and control systems or providing training that does not necessitate full-scale, high-fidelity simulation. To help solve this problem and others, this research project advances theoretical and applied knowledge with regard to alarm systems at nuclear power plants (NPPs). The focus of the research is to develop the first principles of alarm management that will help establish the technical basis for the design of new alarm systems at NPPs. This project covers two steps toward improved alarm systems:

1. Evaluation of alarm management system technologies. INL and the University of Idaho (UI) have reviewed various alarm management system principles and technologies and have deployed them on a simulator. In addition, the research team has developed a plan for testing operator performance with these systems.

2. Deployment of a microworld simulator model for NPP process control. A "microworld" simulator represents a simplified form of the process control system that may be tailored to less skilled operators. For research, more efficient use of limited resources could be realized by using these lower-fidelity microworld simulations, allowing DOE researchers and their collaborators to focus on particular display and control issues (e.g., decision making and prioritization of actions in response to alarms) using a low-cost simulation that mimics the perceptual, cognitive, and action processes of real-world process control in a more tightly controlled microworld environment. An additional advantage of the microworld environments is that ideas for novel displays and controls can be examined in a simplified context that does not necessarily require trained operators to act as participants. Novel display and control concepts can be tested quickly and efficiently using the microworld, and those concepts that show promise can then be further tested using full-scale, high-fidelity simulation with trained operators. In addition, the flexibility of the microworld can provide trainees a view of the internal dynamics of a process control system much more efficiently than using a full-scale, high-fidelity, control room simulation.

\section{Summary}

Microworld Development. Two related software tools, a visual Microworld Scenario Editor and a Microworld Scenario Simulator (the player), have been developed for training and research using Oracle's platform-independent Java language. The Editor provides a visual tool in which a variety of system components can be visually created and connected using a drag-and-drop visual interface. Figure 1 shows an example of a simple interconnected set of pumps, pipes, valves, and reservoirs designed in the Microworld Scenario Editor.

Each component is created by selecting it from the menu and then clicking in the display area to place the component. Once placed, the components can be linked, moved, resized, and deleted by right-clicking on them and selecting these commands from a pop-up menu. System components particular to a pressurized water

\footnotetext{
${ }^{1}$ University of Idaho
} 


\section{CENTER FOR ADVANCED ENERGY STUDIES}

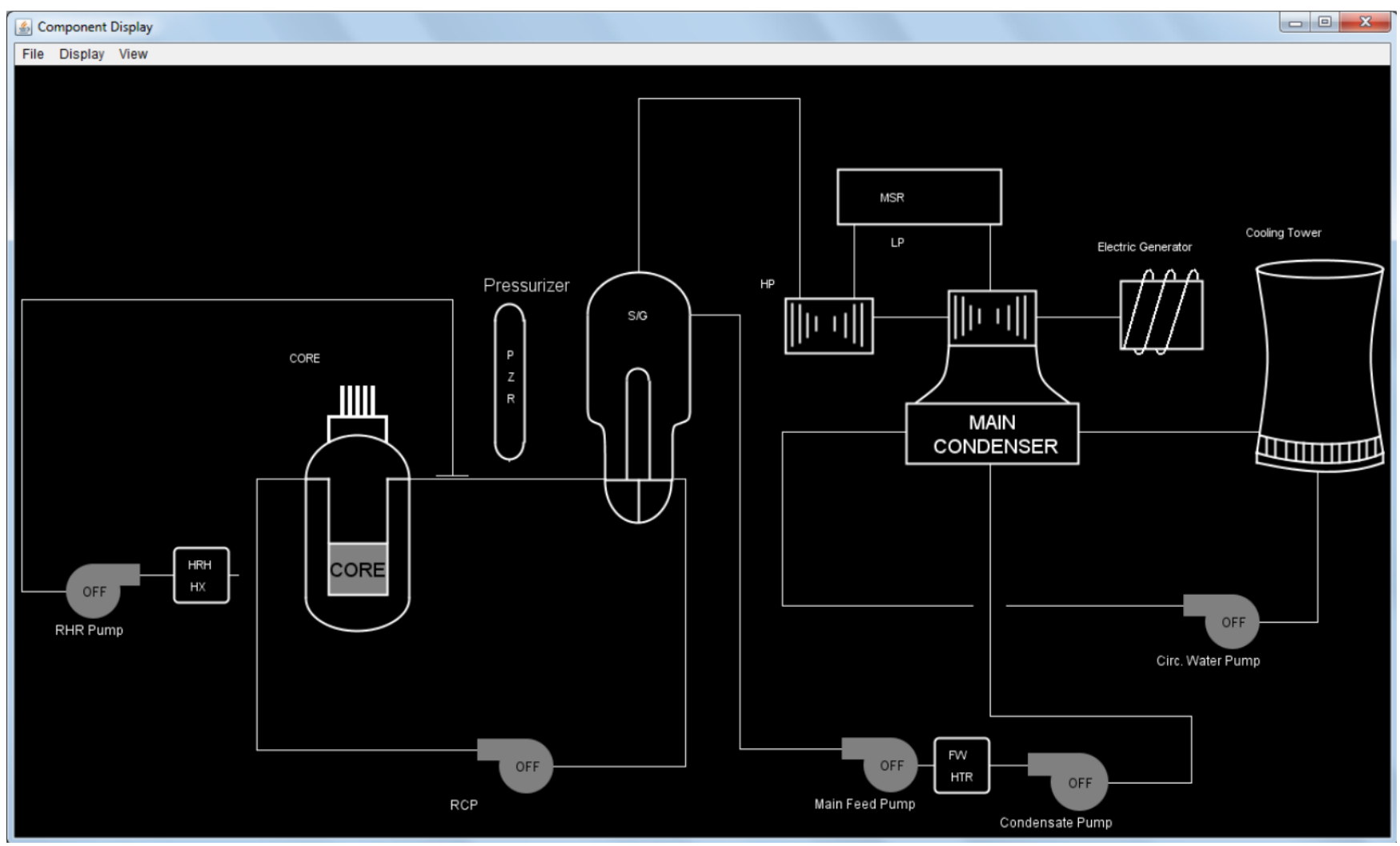

Figure 1. A simple process control microworld shown in the editor window.

reactor in an NPP are also available, as illustrated in Figure 1. While the current objects are customized for nuclear processes, the tool set is flexible and can be used for other process control applications like chemical manufacturing or oil and gas production and distribution. It is also possible to configure the objects to represent a grid, which makes future applications such as smart grids an easy extension.

A configuration editing window can also be opened when a component is right-clicked. The component configuration window allows the simulation designer to set parameter values, parameter and variable displays, parameter controls, set-point alarms of various types, and disturbances (e.g., failures such as external repeating disturbances defined by various waveforms). These can be set independently for all components.

The displays for system variables and the displays and controls for component parameters can be visually arranged within their own dedicated display window using a drag-and-drop interface. Tiled alarm panels can be created by turning on the alarms and creating set points in the component configuration window. These alarm tiles are displayed in a specialized alarms window.

Simulation designers can use the visual interface to lay out system components and configure some of the simpler components (pipes, valves, and reservoirs) with controls, displays, and alarms. Developed scenarios can be saved as Extensible Markup Language-formatted files and reopened. The Microworld Scenario Simulator can read in these scenario files and display the system components in a visually dynamic display (e.g., reservoirs fill and flush, pumps turn on, and alarms change state based on set points being exceeded). Once a simulation 
scenario has been created, it can be played using the Microworld Scenario Simulator. A typical scenario would display the system overview screen, the displays and controls screen, and the alarms screen, which the designer can place on different physical screens depending on the hardware used to run the simulator. The Scenario Simulator provides logging of all system component variables, parameters, and user inputs, affording both offline analysis and variable-speed playback of operator interactions with the simulation.

\section{Benefits to DOE}

DOE currently uses control rooms to control generation, transmission, and distribution of power sources. Each control room features alarm systems. While the focus of this research was primarily on alarm systems in NPPs, this project developed a set of alarm research methods that can be used across energy sectors to produce more effective alarms. This research established a core competence within DOE in alarm research for energy applications. It provides a pipeline of competent student researchers from the university setting who can support such research needs in the future.

\section{Publications}

Ragsdale, A., R. Lew, and R. Boring, "Differences in Trust between Two Types of Alarms and Events within a Nuclear Power Plant Control Simulation," Human Performance in Extreme Environments, 2015, in press.

Lew, R., R. Boring, and T. Ulrich, "A Computerized Operator Support System," $7^{\text {th }}$ International Symposium for Resilient Control Systems, Denver, CO, August 19-August 21, 2014.

Ulrich, T., R. Boring, and R. Lew, "A Computerized Operator Support System," Human Factors and Ergonomics Society $58^{\text {th }}$ Annual Meeting, Chicago, IL, October 27-October 31, 2014.

Dyre, B., et al., "A Microworld Simulator for Process Control Research and Training," Proceedings of the Human Factors and Ergonomics Society 57th Annual Meeting, San Diego, CA, September 30-October 4, 2013.

Ragsdale, A., et al., "Fault Diagnosis with Multi-state Alarms in a Nuclear Power Control Simulator," Proceedings of the Human Factors and Ergonomics Society 56th Annual Meeting, Boston, MA, October 22-26, 2012.

Boring, R., and J. Persensky, "Hybrid Alarm Systems: Combining Spatial Alarms and Alarm Lists for Optimized Control Room Operation," 8th International Topical Meeting on Nuclear Power Plant Instrumentation, Control, and Human-Machine Interface Technologies, San Diego, CA, July 22-26, 2012.

Ragsdale, A., et al., "Alarm Strategy and Complexity: Predictions of Operator Response," 8th International Topical Meeting on Nuclear Power Plant Instrumentation, Control, and Human-Machine Interface Technologies, San Diego, CA, July 22-26, 2012.

Boring, R., et al., "Microworlds, Simulators, and Simulation: Framework for a Benchmark of Human Reliability Data Sources," Joint Probabilistic Safety Assessment and Management and European Safety and Reliability Conference, Scandic Marina Congress Center, Helsinki, Finland, June 25-29, 2012.

Ragsdale, A., Nuclear Power Plant Operator Performance when Systems Vary in Alarm Type, Alarm Accuracy, and Information Type, M.S. Thesis, University of Idaho, 2012. 


\section{Presentations}

Ragsdale, A., R. Lew, and R. Boring, "Differences in Trust between Two Types of Alarms and Events within a Nuclear Power Plant Control Simulation," Resilience Week, San Francisco, CA, August 13-15, 2013. 


\title{
13-011 - Integrated Approach to Algal Biofuel, Bio-power, and Agricultural Waste Management
}

\author{
Deborah T. Newby, Erik R. Coats, ${ }^{1}$ Kevin Feris, ${ }^{2}$ Armando McDonald, ${ }^{1}$ and Brad Wahlen
}

Over nine million dairy cows in the U.S. generate $>226$ billion $\mathrm{kg}$ of wet manure and produce $>5.8$ billion $\mathrm{kg}$ of $\mathrm{CO}_{2}$ equivalents annually. In the anaerobic digestion (AD) process, animal manure is treated by natural bacteria in the absence of oxygen to produce methane-rich biogas that can be captured and used for the production of electricity or heat. Integrated with $A D$, residuals (upstream/downstream) can be separated and used for various purposes, including fertilizer, animal bedding, polyhydroxyalkanoates (PHAs, which are biological, biodegradable thermoplastics with significant commercial value), and algae cultivation, as shown in the process flow diagram in Figure 1.

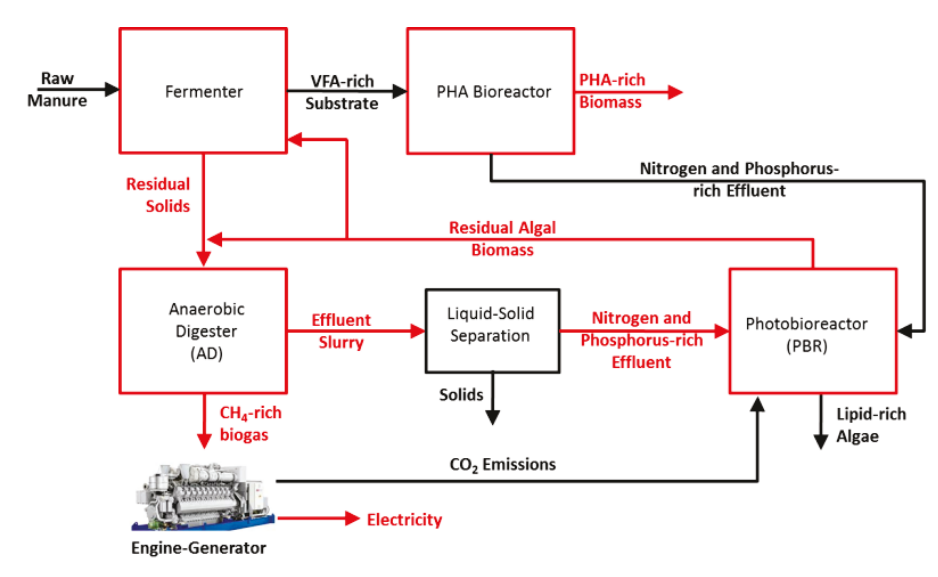

Figure 1. Process flow diagram for the integrated AD-Algal operations, (N/P-rich effluent streams are highlighted in red).

While $A D$ is a mature technology, its growth is stunted due to poor economics and the need to further mitigate the release of emissions (e.g., nitrogen $[\mathrm{N}]$, phosphorus $[\mathrm{P}], \mathrm{CO}_{2}$ ) into the environment. While these nutrients are problematic for the dairy industry, they are of significant value for large-scale cultivation of algal biomass for biofuels and bioenergy. The goal of this project is to build upon and scale up prior work that has established the effectiveness of deploying a novel two-stage $A D$ system coupled with an open algal photobioreactor to produce multiple value-added commodities (bio-power, biofuels, PHA, etc.). This research differs from a conventional algal biofuel approach in that we incorporate a strategy to minimize costly nutrient import and reduce water use by connecting algal production to dairy wastewater treatment. Outcomes of this work will simultaneously enhance $A D$-algal economics while producing valuable end-products and reducing nutrient emissions from animal waste management systems. Coupling the generation of additional product streams to an established industry that demands enhanced waste/emissions management will facilitate enhanced AD-algae biofuel adoption by addressing key barriers as well as enhancing commercial viability and deployment.

\section{Summary}

Significant progress has been made during FY 2014. Three 100-L algal cultivation systems constructed in the Boise State University greenhouse have been used for 45-60 day trials during which various operational conditions using both the anaerobic digester effluent (ADE) and polyhydroxyalkanoate effluent (PHAE) as inputs were evaluated. PHA and AD reactors, fed a dairy manure-based substrate, are being continuously operated in Dr. Coats' laboratory to provide Dr. Feris with PHAE and ADE for his algal investigations. To support Dr. Feris' algal studies, Dr. Coats has collected and provided $>200 \mathrm{~L}$ of PHAE and $>20 \mathrm{~L}$ of ADE. Dr. Newby serves as the project manager and provides technical contributions through routine review of research progress and results.

\footnotetext{
${ }^{1}$ University of Idaho

${ }^{2}$ Boise State University
} 
Growth rates and yield of $C$. vulgaris varied by effluent concentration and type, with high concentrations of both ADE and PHAE resulting in higher yields, likely due to additional nutrient availability (Figure 2). Biomass generated through these experiments has been characterized in terms of lipid and biomass quantity and evaluated as feedstock for internal recycle. Experiments are also being performed to understand the impacts of substrate quality influences on algal production. Although higher initial nutrient concentrations (i.e., higher effluent concentrations) resulted in higher biomass yields, measures of algal lipid content suggest an inverse relationship with initial effluent concentration, a response that is likely a function of hydraulic retention time and the duration of nutrient limitation during stationary phase experienced in our semi-continuous cultivation system. Indeed, N-removal, as percent nitrate removed, was unaffected by initial effluent concentration, suggesting that differences in relative algal lipid content were not due to the presence of residual $\mathrm{N}$ but rather the duration of $\mathrm{N}$-limitation (Figure 2).

Given that changes in initial effluent concentrations alter more factors than available N (e.g., optical properties, available $\mathrm{P}$, concentration of other microbial competitors, and grazers), controlled experiments are currently underway to simultaneously assess the relative effect of initial $\mathrm{N}$ content and optical properties on yield and biomass composition. Specifically, we are investigating the relative importance of total $\mathrm{N}, \mathrm{N}$ species, and substrate optical properties (specifically influence of ultraviolet [UV] pre-treatment) on algal biomass yield and growth rates of a range of different species. Initial results support previous bench scale experiments, indicating that UV-pretreatment can enhance the growth rate of $C$. vulgaris in ADE under semi-continuous operation in the 100-L raceways (Figure 3 ). Year 3 experiments will explore the potential influences of these factors on yields and stability of algal polycultures grown in batch and semi-continuously on ADE and PHAE.

The viability of algal biomass as a fermenter and/or $A D$ substrate to improve volatile fatty acids, $\mathrm{PHA}$, and/or $\mathrm{CH}_{4}$ production is also under investigation. Three sets of paired fermenter-AD reactors were operated and evaluated for approximately 120 days. One set of reactors received only (a)
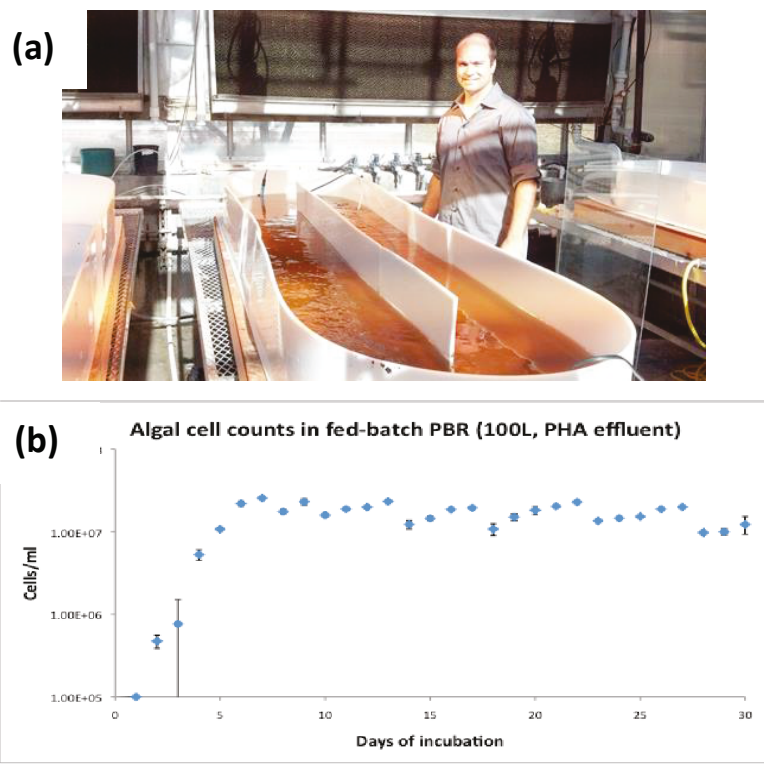

(c)

\section{C. vulgaris growth rate, biomass, \& lipid}

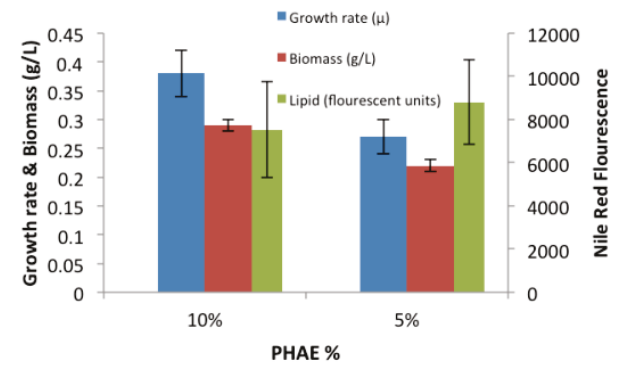

(d)

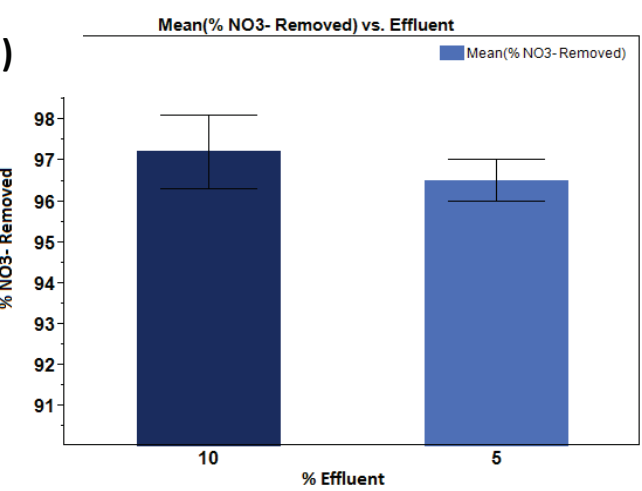

Figure 2. (a) Algal raceways in the Boise State University greenhouse (Jeric Harper, Boise State University Master of Science student shown).

(b) Typical growth response of C. vulgaris during continuous operation. (c) Yield, growth rate, and relative lipid content of $\mathrm{C}$. vulgaris grown under semi-continuous raceway operation. (d) Nutrient removal under same conditions. 


\section{CENTER FOR ADVANCED ENERGY STUDIES}

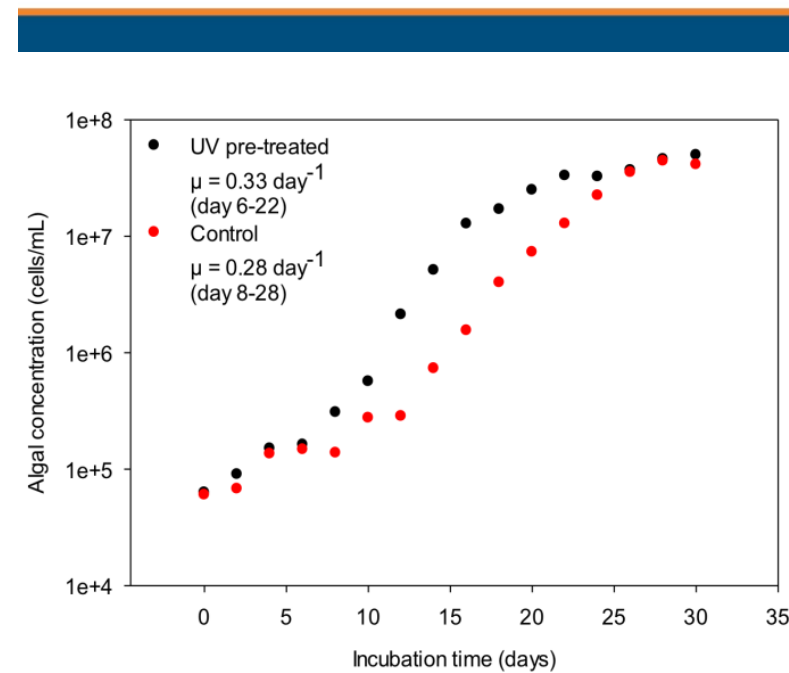

Figure 3. Effect of UV-pretreatment of ADE on C. vulgaris growth in raceways. dairy manure, while a second set received algae ( $10 \%$ manure offset, volatile solids basis) into the fermenter and a third set received algae (10\% manure offset, volatile solids basis) into the AD. The following parameters were monitored: volatile solids destruction, volatile fatty acid production, and biogas $/ \mathrm{CH}_{4}$ production. In addition, the AD culture was characterized for methanogenic populations using the real-time polymerase chain reaction, and algal biomass freeze/thaw was evaluated to improve biodegradability and provide a more high-value substrate. Preliminary assessments (Table 1) indicate that the algal biomass was readily digestible and represents a useful supplement for biogas and/or PHA production. Data analysis and synthesis are currently being finalized, and this research has generated a peer-reviewed publication. Funding supported a part-time postdoctoral scholar and a graduate student at the University of Idaho.

Table 1 - Algal Biomass Fermentation/Anaerobic Co-digestion Results

\begin{tabular}{|c|c|c|c|c|c|c|}
\hline \multirow{2}{*}{$\begin{array}{c}\text { Fermenter (4-day HRT) } \\
\text { Feed }\end{array}$} & \multicolumn{2}{|c|}{ Influent } & \multicolumn{4}{|c|}{ Fermenter Performance } \\
\hline & $\begin{array}{c}\text { Manure VS } \\
(\mathrm{g} / \mathrm{L})\end{array}$ & $\begin{array}{c}\text { Algae VS } \\
(\mathrm{g} / \mathrm{L})\end{array}$ & VFA produced (mgVFA/gVS fed) & $\begin{array}{c}\text { Manure VFA } \\
\text { (mgVFA/gVS fed) }\end{array}$ & $\begin{array}{c}\text { Algae VFA } \\
\text { (mgVFA/gVS fed) }\end{array}$ & $\mathrm{pH}$ \\
\hline Manure (Control) & 37.6 & 0.0 & $77 \pm 21$ & 77 & - & 6.23 \\
\hline Manure and Algae ( $9: 1$ by VS) & 33.9 & 3.6 & $79 \pm 20$ & 78 & 75 & 6.08 \\
\hline Manure (Control) & 37.6 & 0.0 & $83 \pm 22$ & 83 & - & 6.21 \\
\hline
\end{tabular}

\begin{tabular}{|c|c|c|c|c|c|c|}
\hline \multirow{2}{*}{$\begin{array}{c}\text { Anaerobic Digester (20-day HRT) } \\
\text { Feed }\end{array}$} & \multicolumn{2}{|c|}{ Influent } & \multicolumn{4}{|c|}{ AD Performance } \\
\hline & $\begin{array}{c}\text { Manure VS } \\
(\mathrm{g} / \mathrm{L})\end{array}$ & $\begin{array}{c}\text { Algae VS } \\
(\mathrm{g} / \mathrm{L})\end{array}$ & $\mathrm{CH} 4$ produced (ml/gVS fed) & $\begin{array}{l}\text { Manure } \mathrm{CH} 4 \\
\text { (ml/gVS fed) }\end{array}$ & $\begin{array}{l}\text { Algae } \mathrm{CH} 4 \\
\text { (ml/gVS fed) }\end{array}$ & $\begin{array}{c}\mathrm{CH} 4 \% \text { in } \\
\text { Biogas }\end{array}$ \\
\hline Fermented Manure (Control) & 63.8 & 0.0 & $200 \pm 29$ & 200 & - & $58.4 \%$ \\
\hline Fermented Manure \& Algae & 56.8 & 6.7 & $189 \pm 22$ & 203 & 37 & $62.4 \%$ \\
\hline Fermented Manure \& Raw Algae & 56.8 & 9.7 & $203 \pm 24$ & 203 & 198 & $63.5 \%$ \\
\hline
\end{tabular}

To complement the above-described work progress, during the last quarter of the FY, a new task focused on development of near-infrared and Fourier transform infrared spectroscopy to monitor lipid content of algal biomass in real-time was added along with an associated budget increase. The technique will ultimately be expanded to include analysis of other algal biomass components, allowing rapid assessment of biomass quality that may be used to inform downstream conversion processes. The analysis capability developed here will ultimately be applied to track specific algal populations within a mixed community. A new postdoctoral position was filled at INL to advance this aspect of the research.

The project has also facilitated work force development, partnerships, and future commercialization of technologies. Two graduate students, three undergraduate students, a part-time postdoctoral scholar, and a research technician have all been supported to advance this research. A manuscript was submitted to Algal Research on UV pre-treatment of dairy waste and was accepted in September. We have submitted another 
manuscript to Bioresource Technology (titled "Residual Algal Biomass as a Biogas and PHA Biorefinery Substrate"). In addition, the team has supported iSTEM efforts by delivering a series of talks and demonstrations on algal biofuels at multiple sites in Idaho (Lewis-Clark State College, North Idaho College, Eastern Idaho Technical College, College of Southern Idaho, and College of Western Idaho). In addition, a poster was presented in mid-September at the Sustainable Western Dairy and Related Industries workshop where we interfaced with stakeholders, potential industry partners, representatives from potential funding agencies (United States Department of Agriculture, Environmental Protection Agency, World Wildlife Fund), and other key researchers.

\section{Benefits to DOE}

In summary, this research advances algae technologies by using waste streams as a nutrient source, thereby making broader implementation of commercial-scale algal cultivation for fuels more economically feasible. This research also addresses barriers to wider-scale deployment of $A D$, which is presently under-used due to un-optimized systems and under-developed opportunities. New knowledge on a more comprehensive and integrated waste-to-fuels process is needed by the dairy/waste processing industry to improve our understanding of biological processes for processing organic matter. Further, this effort will provide the basis to build strong industrial partnerships by taking this technology from bench-scale to the demonstration level. By integrating education and work force development, skilled bio-product innovators and future system operators will be trained.

\section{Publications}

Prior, M., B. Cragin, A. Hall, N. Staley, E. Coats, A. McDonald, and K. Feris, "Ultraviolet Radiation Pre-treatment Modifies Dairy Wastewater, Improving Its Utility as a Medium for Algal Cultivation," Algal Research, accepted, 2014.

Smith, S., E. Huges, E. Coats, C. Brinkman, K. Feris, J. Harper, and D. Newby, "Residual Algal Biomass as a Biogas and PHA Biorefinery Substrate and Some Causes of Algae Digestion Variations," Bioresource Technol., submitted, 2014.

\section{Presentations}

Feris, K., E. Coats, A. McDonald, B. Wahlen, and D. Newby, "Integrated Approach to Algal Biofuel, Biopower, and Agricultural Waste Management," Sustainable Western Dairy Workshop, Twin Falls, ID, September 16-17, 2014. 


\title{
13-033 - Magnetic Separation Nanotechnology for Spent Nuclear Fuel Recycle
}

\author{
Leigh R. Martin, You Qiang, ${ }^{1}$ and Yaqiao $\mathrm{Wu}^{2}$
}

The goal of this project is to develop an understanding of the usefulness of functionalized magnetic nanoparticles (MNP's) in used nuclear fuel separations, in particular minor actinide $\left(\mathrm{An}^{3+}\right) /$ lanthanide $\left(\mathrm{Ln}^{3+}\right)$ separations although this has also been expanded to include Tc separations. The project will characterize the physical and chemical properties of the ligand-functionalized MNPs (size, shape, aggregation, magnetization, etc.) using electron microscopy, TEM, vibrating sample magnetometer, LEAP and simple solid liquid partitioning experiments

\section{Summary}

\section{Part 1: Study on sorption-stripping cycles}

The ability to reuse the double coated magnetic nanoparticles (dMNP)-diethylenetriaminepentaacetic acid (DTPA) conjugates was investigated by performing up to 15 cycles of sorption/desorption tests. A three-step washing/stripping process using either $0.1 \mathrm{M} \mathrm{HNO}_{3}$ or $1 \mathrm{M} \mathrm{HCl}$ as the stripping agent was developed. As shown in Figure 1, for both cases, over $98 \%$ of cadmium (Cd) was recovered by the first two acid stripping steps, and there was a negligible amount of $\mathrm{Cd}$ after the third deionized water washing. No decrease in sorption efficiency was observed during 15 sorption/desorption cycles.
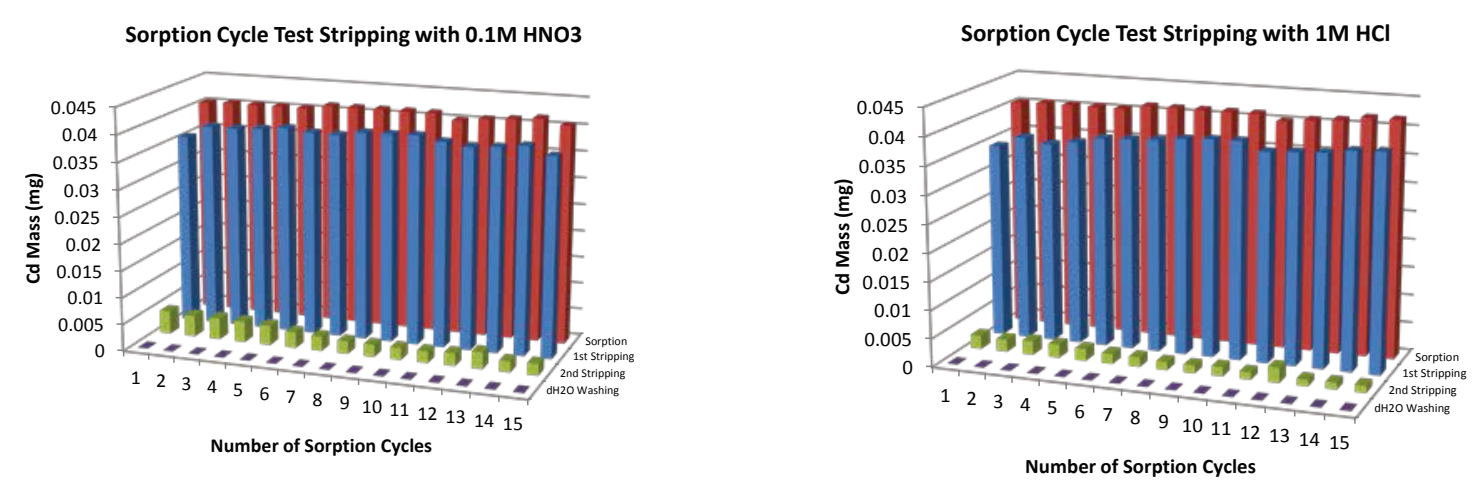

Figure 1. Mass of extracted and back-extracted Cd during 15 sorption/stripping cycles with dMNP-DTPA conjugates.

\section{Part 2: Study on technetium (Tc) sorption}

A preliminary technetium (Tc) sorption study was performed using a tracer level of $\mathrm{TcO}^{4-}, \mathrm{Tc}(\mathrm{IV} / \mathrm{V})$, and $\mathrm{Tc}(\mathrm{CO})^{3+}$ in aqueous solution. As shown in Figure 2, Tc(IV/V) is the most reactive species with either single coated MNP (sMNP) or dMNP-DTPA conjugates with over $95 \%$ removal efficiency. For $\mathrm{TcO}^{4-}$ and $\mathrm{Tc}(\mathrm{CO})^{3+}$ sorption, although the sorption efficiency was not as high as Tc(IV/V), positive sorption was observed. The sorption efficiency could be improved by adjusting the reaction conditions.

\footnotetext{
${ }^{1}$ University of Idaho

${ }^{2}$ Boise State University
} 


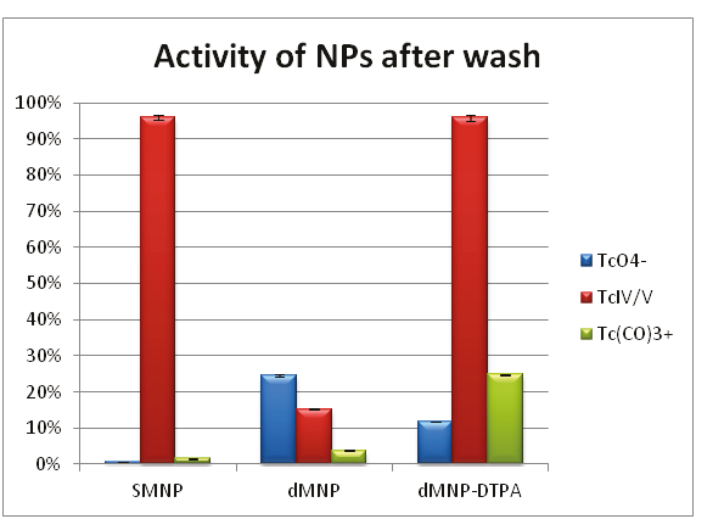

Figure 2. Activity of TC on the particles (sMNP, dMNP, and dMNP-DTPA) after 1 hour of sorption contact time.

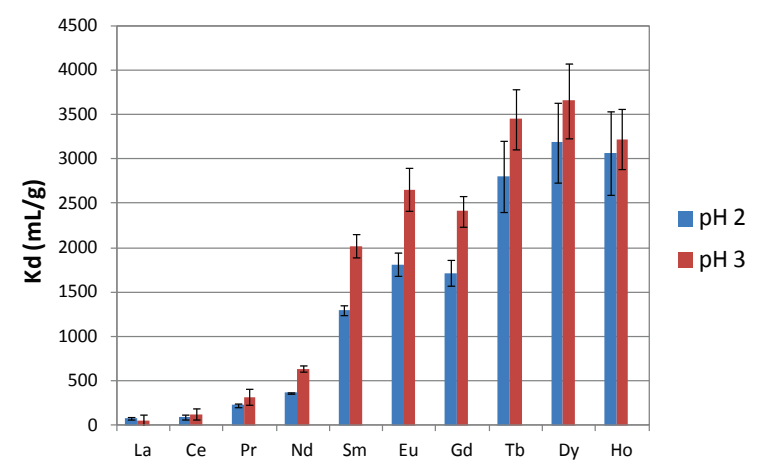

Figure 3. Effects of $\mathrm{pH}$ and atomic number on the sorption efficiency (after 2 hours of contact time).

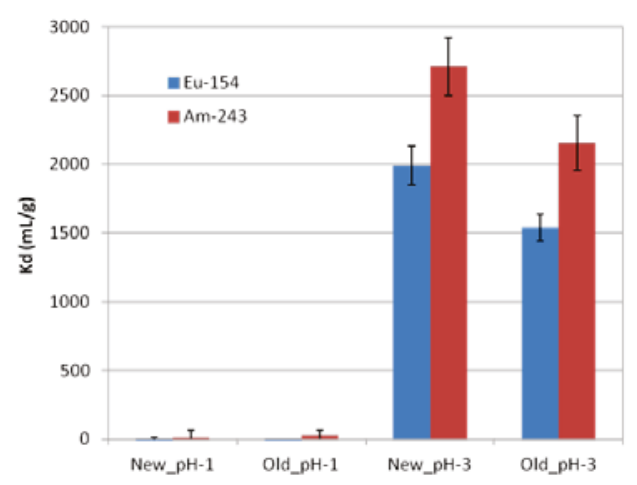

Figure 4. Sorption efficiency of Am(III) and Eu(III) on new and old dMNP-DTPA conjugates under $\mathrm{pH} 1.0$ and $\mathrm{pH} 3.0$ (after 2 hours of contact time).

\section{Part 3: Study on trivalent lanthanide (Ln[III]) sorption}

Sorption experiments on dMNP-DTPA conjugates were conducted for the lanthanide series ( $\mathrm{La}-\mathrm{Ho}$, with the exception of $\mathrm{Pm}$ ). Sorption kinetics and the effect of $\mathrm{pH}$ were evaluated. Ln(III) sorption equilibrium was reached after 30 min of contact time. The sorption efficiency increased across the lanthanide series, which is an electrostatic effect due to the $\mathrm{Ln}$ contraction wherein the ionic radii of the $\mathrm{Ln}(\mathrm{III})$ decrease with an increase in the atomic number, as shown in Figure 3.

Part 4: Study on trivalent americium (Am(III)) and trivalent europium (Eu(III)) separation

Metal sorption experiments onto dMNP-DTPA conjugates were conducted for the $\mathrm{Ln}$ (III) series (La-Ho, with the exception of $\mathrm{Pm}$ ) spiked with trivalent americium (Am)-243 and trivalent europium (Eu)-154. Freshly made dMNP-DTPA (new) and dMNP-DTPA after 6 months of storage (old) were used to evaluate the sample degradation. After 2 hours of contact time, the $K_{d}$ value was $2700 \mathrm{~mL} / \mathrm{g}$ for Am-243 and 2000 mL/g for Eu-154 in a pH 3.0 buffer, as seen in Figure 4. At $\mathrm{pH} 1.0$, there was no sorption on dMNP-DTPA conjugates for both cases. The separation radio of $\mathrm{Eu}(\mathrm{III}) / \mathrm{Am}$ (III) was only $\sim 0.7$. Future study is needed to increase the separation radio.

\section{Part 5: Stability of magnetic nanoparticles under Irradiation.}

Under the project, a portion of research was focused on investigating the irradiation-induced modification in nanomaterials through the understanding of stability and sensitivity of nanomaterials under irradiation. Our investigation of $\mathrm{Si}^{2+}$ ion-irradiation (5.5 MeV to fluence of 1016 ions/cm2) on core-shell iron (Fe)-Fe oxide nanoparticle granular films has brought forth some interesting results resisting irradiation as well as phase change. These core-shell nanoparticle granular films continued to remain ferromagnetic with the core unaffected even after irradiation. The grain size of the $\mathrm{Fe}(\sim 8 \mathrm{~nm})$ core remained unchanged, with only the growth of shell size from $2 \mathrm{~nm}$ to $17 \mathrm{~nm}$. The oxide shell of these nanoparticles had stoichiometry change from $\mathrm{Fe}_{3} \mathrm{O}_{4}$ to $\mathrm{FeO}$ under irradiation and the new $\mathrm{Fe}-\mathrm{FeO}$ interface played a vital role in protecting the core. It has also been found that valence reduction of Fe oxides happen only 
if pure nanocrystalline $\mathrm{Fe}$ is present; otherwise no reduction behavior was observed. This research finding contributes to enhancing the stability of materials through stoichiometry and nanomaterials synthesis.

\section{Part 6: TEM Characterization of MNPs.}

Nano-sized magnetic powder samples coded MNP, sMNP, dMNP, dMNP-DTPA, and dMNP-DTPA-Ln were characterized by using the transmission electron microscopy (TEM) technique. Figure 5 shows representative microstructures of the samples. In addition, their chemical compositions were confirmed by using the energy dispersive X-ray spectroscopy technique. The TEM images clearly show the successive increase in thickness of coating on MNPs by different surface functionalities. The thickness of the first silica coating is $\sim 40 \mathrm{~nm}$ (Figure $5 \mathrm{~b}$ ). After a second coating, the size of particles with polymer structure (silica and polyamine) significantly increased to micro-scale (Figure $5 \mathrm{c}$ ). The first silica coating was still clearly recognizable after the second coating (Figure 5d). The DTPA attachment did not have a significant effect on the particle size and surface structure (Figures $5 \mathrm{e}$ and $5 \mathrm{f}$ ). The TEM images also revealed that the first silica coating was not coated on the individual Fe/Fe oxide MNPs. Instead, the MNPs agglomerated before the surface coating. Another issue with the conventional TEM analysis is that it requires dry samples. However, the size and surface structure of dMNP and dMNP-DTPA particles significantly changed after dry due to the water-absorbing polymer structure of polyallylamine on the second coating. Therefore, other imaging techniques will be needed that allow performing the sample analysis in an aqueous solution.

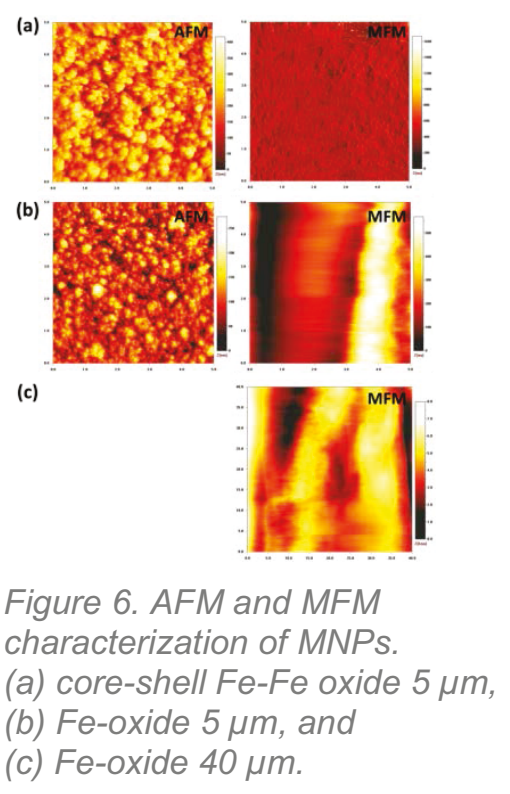

\section{Part 7: AFM/MFM characterization of MNPs}

Atomic force microscopy (AFM) and magnetic force microscopy (MFM) were employed to characterize core-shell Fe-Fe oxide $5 \mu \mathrm{m}$, Fe-oxide $5 \mu \mathrm{m}$, and Fe-oxide $40 \mu \mathrm{m}$ magnetic nano-sized powder samples. The AFM images give surface morphology of the $5 \mu \mathrm{m}$ MNPs (Figures $6 \mathrm{a}, 6 \mathrm{~b}$ ). In the AFM images, the brighter contrast means higher height of the particles. For example, the brightest (white color) contrast represents a height over $400 \mathrm{~nm}$. MFM is useful to show magnetic domains in the sample.

The boundaries between bright and darker contrast areas in the MFM images of Fe-oxide samples (Figures $6 \mathrm{~b}$ and $6 \mathrm{c}$ ) should represent the magnetic domain boundaries in the two samples.

\section{Part 8: Future study plan}

The Tc sorption efficiency will be improved by adjusting the reaction conditions, such as changing the $\mathrm{pH}$, increase the amount of particles, or conducting the tests at macro scale, etc. The separation radio of Eu/Am will be improved either by oxidizing $A m(I I I)$ to higher oxidation states $(A m(V / V I))$ to 
reduce the complexing reaction between Am and DTPA or by conjugating a kind of new metal chelator that has a high sorption selectivity to $\mathrm{Ln}(\mathrm{III})$ over trivalent actinide (Am(III)), such as Di-(2-ethylhexyl)phosphoric acid, to the surface functionalized MNPs. Magnetic separator design for the continuous operation will be continued.

\section{Benefits to DOE}

The study of MNPs for minor actinide/ lanthanide and technetium separations is providing data on the long term performance of these magnetic materials in potential radioactive materials remediation applications. This work is contributing to the design of an advanced fuel cycle that is proliferation resistant.

\section{Publications}

Sundararajan, J. , M. Kaur, W. Jiang, J. McCloy, and Y. Qiang, "Oxide Shell Reduction and Magnetic Property Changes in Core-Shell Fe Nanoclusters under Ion Irradiation," J. Appl. Phys., Vol. 115, 17B507, 2014.

Kaur, M., H. Zhang and Y. Qiang, "Magnetic Separation Dynamics of Colloidal Magnetic Nanoparticles," Magnetics Letters, IEEE, Vol.4, 4000204, 2013.

Kaur, M., Y. Qiang, et al., "Separation Nanotechnology of Diethylenetriamine-Pentaacetic Acid Bonded Magnetic Nanoparticles for Spent Nuclear Fuel," Nano Energy, Vol. 2, 124-132, 2013.

Kaur, M., H. Zhang, L. Martin, T. Todd, and Y. Qiang, "Conjugates of Magnetic Nanoparticle-Actinide Specific Chelator for Radioactive Waste Separation," Environmental Science \& Technology, Vol. 47, 11942-11959, 2013.

Qiang, Y., M. Kaur, H. Zhang, L. Martin, and T. Todd, "Magnetic Separation-Advanced Nanotechnology for Future Nuclear Fuel Recycle," ANS Global 2013, Salt Lake City, UT, September 29-October 3, 2013.

Zhang, H., M. Kaur, and Y. Qiang, "Magnetic Nanosorbents for Fast Separation of Radioactive Waste," ANS Global 2013, Salt Lake City, UT, September 29-October 3, 2013.

McCloy, J., W. Jiang, T. Droubay, T. Varga, E. Burks, J. Sundararajan, M. Kaur, Y. Qiang, L. Kovarik, and K. Liu, "Ion Irradiation of Fe-Fe Oxide Core-Shell Nanocluster Films: Effect of Interface on Stability of Magnetic Properties," J. Appl. Phys., Vol. 114, 083903, 2013.

\section{Presentations}

Wu, Y., M. Kaur, H. Zhang, Y. Qiang, L. Martin, and T. Todd, "Magnetic Cr-doped Fe-Fe Oxide Core-Shell Nanoparticles for Used Nuclear Fuel Separation," TMS 2015, accepted.

Kaur, M., Y. Wu, H. Zhang, L. Martin, T. Todd, and Y. Qiang, "Nanoparticle for Used Nuclear Fuel Separation," TMS 2014, San Diego, CA, February 16-20, 2014. 


\section{3-035 - Development of New Molten Salt Sensor Technology for Application to Safeguarding Pyroprocessing}

Ken Bateman, Robert Hoover, Michael Shaltry, Michael Simpson, ${ }^{1}$ Kelly Allahar, ${ }^{2}$ Darryl Butt, ${ }^{3}$ Supathorn Phongikaroon, ${ }^{3}$ and Abraham Jurovitzki ${ }^{2}$

There is an increasing need for DOE to safeguard its pyroprocessing facilities. Current state-of-the-art sensors are insufficient to provide real-time process monitoring of electrorefiners suitable to prevent diversion of fissile nuclear materials. This project involves the experimental investigation of various sensor technologies for application in monitoring the state of molten salt used for electrorefining in a pyroprocessing operation. Two different types of sensors have been investigated-electrical impedance spectroscopy (EIS) and ultraviolet-visible (UV-Vis) spectroscopy. Application of these methods to molten salts is relatively new and involves significant challenges.

\section{Summary}

For investigation of EIS, the first year included experiments with several different rare earth or actinide salts in molten $\mathrm{LiCl}-\mathrm{KCl}$. This included $\mathrm{UCl}_{3}, \mathrm{ZrCl}_{4}, \mathrm{LaCl}_{3}$, and $\mathrm{CeCl}_{3}$. Tests were run in the Radiochemistry Laboratory of CAES as a collaborative effort between Boise State University and the University of Idaho. Signatures were recorded to distinguish between different species in the salt, and modeled time constants were found to depend on both the ion being measured and its concentration. These initial findings were presented at the 2013 TMS Meeting.

In the second year, more in-depth investigation was made of the $\mathrm{LiCl}-\mathrm{KCl}-\mathrm{SmCl}_{3}$ system, which involves a soluble-soluble transition $\left(\mathrm{Sm}^{3+} / \mathrm{Sm}^{2+}\right)$. Molten salt solutions consisting of eutectic $\mathrm{LiCl}-\mathrm{KCl}$ and varying concentrations ( 0.5 to $3.0 \mathrm{wt} \%$ ) of $\mathrm{SmCl}_{3}$ were analyzed at $500^{\circ} \mathrm{C}$ using both cyclic voltammetry (CV) and EIS. For analyzing CV data, the Randles-Sevcick equation was employed. This equation relates CV peak height to concentration but also requires knowledge of the diffusion coefficient. Diffusion coefficients were thus measured by using this equation, $\mathrm{CV}$ data, and known concentrations. The results indicated significant variability in the diffusion coefficient over the range of concentrations, thus suggesting that CV has limited potential for highly accurate analysis of the salt. This motivates the investigation of the EIS method. EIS spectra at the anodic, cathodic, and half-peak potentials were then measured. A six-element Voigt model was used to analyze the EIS data in terms of resistance-time constant pairs. The lowest resistances were observed at the half-peak potential with the associated resistance-time constant pairs characterizing the reversible reaction between $\mathrm{Sm}^{3+}$ and $\mathrm{Sm}^{2+}$. Regressed model parameters were used to extrapolate to the polarization resistance. A polarization resistance-concentration relationship was determined that yielded bulk concentration values similar to experimental values. The clear advantage of this approach is that knowledge of the diffusion coefficients is not necessary to predict concentration based on electrochemical response. In the final year of the project, experiments are planned to be performed at CAES with additional salt mixtures that feature both soluble-soluble and soluble-insoluble transitions ( $\mathrm{LiCl}-\mathrm{KCl}-\mathrm{LaCl}_{3}-\mathrm{SmCl}_{3}, \mathrm{LiCl}-\mathrm{KCl}-\mathrm{CeCl}_{3}$, and $\mathrm{LiCl}-\mathrm{KCl}-\mathrm{UCl}_{3}$ ) to further investigate the potential for using EIS to predict molten salt compositions.

\footnotetext{
${ }^{1}$ University of Utah

${ }^{2}$ Boise State University

${ }^{3}$ Virginia Commonwealth University
} 
For the investigation of UV-Vis spectroscopy, the second year of the project involved setting up a high-temperature system to enable UV-Vis absorption spectroscopy measurements of molten salt. A furnace with two in-line view ports was purchased and installed into an argon $(\mathrm{Ar})$ atmosphere glove box at the University of Utah. The graduate student working on the project, A. Jurovitzki, prepared a number of salt mixtures containing $\mathrm{LiCl}, \mathrm{CsCl}$, and $\mathrm{SrCl}_{2}$. The hypothesis we were testing was that UV-Vis spectroscopy of samples of salt could be used to detect the presence of actinides. In the oxide reduction process, molten $\mathrm{LiCl}$ salt is used, and there should be no actinides in solution unless the process is being misused for diversion of nuclear material. While electrorefiner salt will have countless dissolved ions that could interfere with spectroscopic measurements, the oxide reduction salt will only have $\mathrm{CsCl}, \mathrm{SrCl}_{2}$, and a few other minor species. Actinides stay in the fuel basket unless they are intentionally oxidized. UV-Vis measurements of $\mathrm{LiCl}-\mathrm{CsCl}-\mathrm{SrCl}_{2}-\mathrm{UCl}_{3}$ will be useful to determine the ability and threshold sensitivity to measure $\mathrm{UCl}_{3}$ in the salt. In addition to setting up the furnace, aligning optics, and running baseline tests, UV-Vis spectra were collected for $\mathrm{LiCl}$ with varying concentrations of $\mathrm{CsCl}$ and $\mathrm{SrCl}_{2}$. Since we did not have any $\mathrm{UCl}_{3}$ in the lab, a method was designed to chlorinate $\mathrm{U}_{3} \mathrm{O}_{8}$ in situ to form $\mathrm{UCl}_{3}$ in the salt. This involves reaction of $\mathrm{ZrCl}_{4}$ with the uranium $(U)$ oxide. This reaction is planned for the first quarter of the third year of the project and will be followed by both CV and UV-Vis spectroscopy of the salt. The relative sensitivity and challenges with each method will be compared and contrasted.

\section{Benefits to DOE}

DOE's national security mission is to enhance nuclear security through defense, non-proliferation, and environmental efforts. This project directly contributes to the proliferation need. If successful, this project will deliver key sensor technologies that are anticipated to contribute greatly to solving this problem.

\section{Publications}

Allahar, K., M. Shaltry, D. Butt, M. Simpson, S. Phongikaroon, and K. Bateman, "Electrical Impedance Spectroscopy and Cyclic Voltammetry Methods for Monitoring $\mathrm{SmCl}_{3}$ Concentration in Molten $\mathrm{LiCl}-\mathrm{KCl}$ Eutectic," Electroanalytical Chemistry, in preparation.

\section{Presentations}

Jurovitzki, A. et al., "Monitoring Pyroprocessing through Electrochemical Methods," Annual Meeting of the American Institute of Chemical Engineers, Atlanta, GA, November 17, 2014.

Jurovitzki, A. et al., "Monitoring Fission Products and Actinide Levels during Pyroprocessing via UV-vis Absorption Spectroscopy," International Pyroprocessing Research Conference, Idaho Falls, ID, October 19-23, 2014.

Allahar, K. et al., "Electrochemical Impedance Spectroscopy Monitoring of $\mathrm{SmCl}_{3}$ in $\mathrm{LiCl}-\mathrm{KCl}$ Molten Salt," DOE MPACT Working Group Meeting, San Diego, CA, March 18-20, 2014.

Simpson, M., et al., "Spectroscopic Real Time Monitoring of Molten Salts in Nuclear Electrorefiner Systems," TMS 2014, San Diego, CA, February 20, 2014. 


\section{CENTER FOR ADVANCED ENERGY STUDIES}

Allahar, K., et al., "Electrochemical Impedance Spectroscopy of $\mathrm{LaCl}_{3}, \mathrm{ZrCl}_{4}$, and $\mathrm{UCl}_{3}$ in $\mathrm{Molten} \mathrm{LiCl}-\mathrm{KCl}$ Eutectic," TMS 2013, San Antonio, TX, March 3-7, 2013.

Simpson, M., et al., "Sensor Technology for Real Time Monitoring of Molten Salt Electrolytes during Nuclear Fuel Electrorefining," TMS 2013, San Antonio, TX, March 3-7, 2013. 
13-068 - Cooling in Fractured Geothermal Reservoirs: Analyses of Long-Term Cooling in Typical Geothermal Reservoirs and Application to Geothermal Resource Potential Assessments

\author{
Mitchell Plummer, Jerry Fairley, ${ }^{1}$ John Welhan, ${ }^{1}$ Michael McCurry, ${ }^{2}$ Roy Mink, ${ }^{3}$ and Ian Warren ${ }^{4}$
}

Despite the importance of the heat extraction process to understanding geothermal energy potential, the conceptual and mathematical relationships that describe it are often neglected because of the complexity of the problem. As a result, geothermal resource potential is often described by the heat available in the subsurface rather than the likely recoverable fraction over useful timescales, and little information is available describing how fluid flow constraints, together with fractured reservoir characteristics, act to define energy productivity in a geothermal system. This project uses a combination of semi-analytical and numerical solutions for the coupled fluid flow and heat transport problem to describe how fractured volume and fracture characteristics control long-term energy productivity, thereby providing a means of improving estimates of the nation's geothermal resource potential. Accordingly, we define two objectives for the proposed project:

1. Develop a set of software tools that can be used to examine and predict the long-term thermal evolution and energy productivity of real and hypothetical geothermal reservoirs

2. Conduct analyses of available data describing different geothermal reservoirs to describe long-term productivity under typical operational constraints.

Completion of the proposed research will allow more realistic assessment of recoverable energy in both conventional and enhanced geothermal systems, thus providing better assessment of the "true" value of a geothermal resource. Collaboration with industry representatives will ensure that (a) our activities focus on the highest priority technical need and current limitations and (b) the outcomes and products of our research will be available and useful to geothermal energy practitioners and will positively impact the role of geothermal energy in U.S. energy production.

\title{
Summary
}

This collaborative project, involving staff from INL, two universities, a commercial geothermal energy operator and a geothermal energy consultant, broadly focuses on long-term cooling behavior in geothermal systems and improved means of predicting and assessing cooling behavior. Accomplishments to date include the following:

- Mitch Plummer, working with Rob Podgorney and other developers from INL's MOOSE team, demonstrated a newly developed method of using embedded lower-dimensional objects and meshes to model fractures in a large (e.g., reservoir-scale) rock domain. The capability, applied in INL's subsurface reservoir simulator, FALCON, allows simulations of mass and heat transport at the strongly contrasting size scales of fractured

\footnotetext{
${ }^{1}$ University of Idaho

${ }^{2}$ Idaho State University

${ }^{3}$ Mink GeoHydro, Inc.

${ }^{4}$ U.S. Geothermal, Inc.
} 
rock reservoirs, a critically important aspect of geothermal reservoir modeling. Results were showcased in INL's presentation at the National Laboratory Day on Capitol Hill on September 12, 2014.

- Inspired by anomalous pressure and flow readings obtained during an enhanced geothermal system test at Raft River, Idaho, Dr. Plummer mentored summer intern Isaac Allred in the development of a MATLAB program demonstrating how diurnal temperature variations in enhanced geothermal system injection fluids can be used to provide transient data for essentially continuous reservoir monitoring. Isaac presented the results of his effort, entitled "Injection Test Analysis on the Edge of Precision," at INL's 2014 Summer Intern Poster Session.

- Collaborator Jerry Fairley of the University of Idaho supported a master's level graduate student, Alex Moody, who spent part of the summer term with Dr. Plummer at INL. Mr. Moody assisted Mr. Ronan with development of the MATLAB code to calculate temperature evolution in a fractured rock reservoir and used that program to make preliminary calculations describing the geothermal potential of the Snake River Plain. Mr. Moody also completed an extensive analysis of fracture distribution in rock cores from deep wells in the Snake River Plain and submitted a manuscript on his master's thesis work to a Geological Society of America Special Publication on Geothermal Energy.

- Using a combination of analytical solutions and numerical modeling, Dr. Plummer demonstrated how cold water injection in a geothermal reservoir is likely to impact well injectivity over time and how pressure response in a well may be used to make inferences about hydraulic properties critical to predicting long-term cooling behavior. The results were presented at the 2013 Geothermal Resources Council Annual Meeting (where the presentation received a "GRC Best Presentation Award"), at the 2013 Geological Society of America annual meeting, and at a CAES Geofluids Colloquium.

- To promote better understanding of long-term cooling behavior in fractured reservoirs, Dr. Plummer hired a summer intern from Boise State University to develop a MATLAB interface for a set of semi-analytical solutions to flow and heat transport equations for fracture flow. That geophysics student, Timothy Ronan, successfully completed most of the interface development, learned fundamentals of fluid flow and heat transfer, and presented his work at the Summer Internship Poster Session. Tim was selected as a Boise State University CAES Energy Scholar for his work.

\section{Benefits to DOE}

The development of engineered geothermal systems could play a substantial role in DOE's mission to bring new clean energy sources to market. Such development requires, however, that we have appropriate tools for understanding and assessing reservoir potential. This project aims to provide tools for rapid assessment of the relationships between fluid flow and basic reservoir characteristics in fractured geothermal reservoirs. Such tools are needed as a reference base for understanding, and application of, more sophisticated reservoir simulators. Project results should fill a critical gap in the industry's ability to understand and predict the effects of heat exchange in fractured reservoirs. 


\section{Publications}

Moody, A., J. Fairley, and M. Plummer, "Designing an Engineered Geothermal Reservoir in the Welded Tuffs of the Snake River Plain, Southern Idaho," Geological Society of America Special Papers: Geothermal Energy: An Emerging Resource, in review.

Plummer, M., "Insights into Cold Water Injection Stimulation Effects through Analytical Solutions to Flow and Heat Transport," Geothermal Resources Council Transactions, Vol. 37, 2013.

\section{Presentations}

Welhan, J., M. Gwyn, S. Payne, M. McCurry, M. Plummer, and T. Wood, "The Blackfoot Volcanic Field, Southeast Idaho: A Hidden High-Temperature Geothermal Resource in the Idaho Thrust Belt," SGP-TR-202, Proceedings, Thirty-Ninth Workshop on Geothermal Reservoir Engineering, Stanford University, Stanford, CA, February 24-26, 2014.

Plummer, M., Insights into Cold Water Injection Stimulation Effects through Analytical Solutions to Flow and Heat Transport," Geothermal Resources Council Annual Meeting, Las Vegas, NV, 2013.

Moody, A., J. Fairley, and M. Plummer, "Theoretical Fracturing and Power Production from Low-Permeability Tuffs in the Snake River Plain, Southern Idaho," Paper No. 338-5, 2013 Geological Society of America Annual Meeting, Denver, CO, 2013.

Plummer, M., K. Smith, and R. Podgorney, "Cold Water Injection Effects in Fractured Reservoirs," Paper No. 387-10, 2013 Geological Society of America Annual Meeting, Denver, CO, 2013. 
INL LDRD SNAPSHOT ANNUAL REPORT FY 2014

Author Index 


\section{Author Index}

A

Akers, Doug, 130

Allahar, Kelly, 201

Andrs, David, 32, 73

B

Bai, Xianming, 51, 167, 182

Bakas, Michael, 112, 118

Baldwin, Tom, 87, 153

Ban, Heng, 182

Bateman, Ken, 201

Berry, Ray, 73

Bier, Vicki, 180

Binder, William, 133

Biner, Bulent, 10, 26

Black, Cherrie, 116

Boring, Ronald, 189

Buettner, Gary, 35

Buongiorno, Jacopo, 57

Burgett, Eric, 8, 40

Burns, Doug, 57

Bush, Jason, 153

Butt, Darryl, 201

G

\section{C}

Carney, Kevin, 81

Carroll, Laura, 18, 142

Carroll, Mark, 18, 142

Chernatynskiy, Aleksandr, 182

Chichester, David, 6, 99, 101, 110

Chu, Henry, 112, 118

Chugg, Jonathan, 94

Coats, Erik, 193

Cole, James, 4

Coleman, Justin, 69

Condie, Keith, 45

Corradini, Michael, 180

Couch, David, 84

Crepeau, John, 45

Curtis, Daniel, 185

Hales, Jason, 18, 29, 54

Harp, Jason, 59 
Heaps, Ron, 97

Herbst, Scott, 49, 174

Hill, Tony, 8, 40

Hiromoto, Robert, 22

Hoover, Robert, 201

Hovsapian, Rob, 133, 153

Hruska, Ryan, 116

Huang, Hai, 29, 126

Hurley, Dave, 147

J

Jamison, David, 161

Jewell, Keith, 8, 40

Johnson, Aaron, 108

Johnson, James, 6, 99, 101

Jurovitzki, Abraham, 201

K

Kane, Joshua, 163

Karpeev, Dmitry, 26, 27

Khafizov, Marat, 176

Kinlaw, Mathew, 99, 101, 104

Klett, Mary, 116

Knudson, Darrell, 45

Kohse, Gordon, 13

Kunerth, Dennis, 147

L

Lessing, Paul, 59

Lillo, Thomas, 112

Lo, Brandon, 84, 85, 114, 115

M

Manic, Milos, 116

Mariani, Robert, 4

Martin, Leigh, 81, 108, 197

Martineau, Richard, 37, 73

Mattson, Earl, 139

McBride, Scott, 87

McCurry, Michael, 204

McCutcheon, Jeffrey, 144

McDonald, Armando, 193

McGrath, Christopher, 81
McJunkin, Tim, 120, 147

McNally, Josh, 144

McQueen, Miles, 120

Meher, Subhashish, 142

Mincher, Bruce, 20, 35

Mink, Roy, 204

Moradi, Hussein, 84, 114

Morel, Jim, 37

Morrison, John, 158

Myers, Kurt, 153

$\mathbf{N}$

Nazar, Linda, 171

Neupane, Ghanashyam, 139, 140

Newby, Deborah, 193

Nielsen, Joseph, 22

Nilsson, Mikael, 174

Novascone, Steve, 54

O'Brien, James, 42

O'Brien, Rob, 59

Orme, Christopher, 144

Pak, Joshua, 124

Palmer, Joe, 13

Pantoya, Michelle, 97

Paredis, Christiaan, 133

Pastore, Giovanni, 54

Permann, Cody, 32

Peterman, Dean, 20

Peterson, John, 32

Petkovic, Lucia, 130

Pfeiffer, Jana, 81, 108

Phongikaroon, Supathorn, 201

Plummer, Mitchell, 139, 204

Pollock, Tresa, 142

Prinja, Anil, 61

Qiang, You, 197 
$\mathbf{R}$

Rabiti, Cristian, 37, 57, 61

Ramirez, Sam, 77

Reid, Carol, 87

Rempe, Joy, 13, 45

Rieger, Craig, 120

Rohde, Kenneth, 94

S

Sabharwall, Piyush, 42

Sazhin, Sergiy, 158

Schultz, Michael, 35

Shaltry, Michael, 201

Shy, Daniel, 110

Simpson, Michael, 201

Sintay, Stephen, 2

Slaughter, Andrew, 32

Smith, Curtis, 57, 73

Smith, Kord, 37

Solstad, Steinar, 13, 45

Spencer, Benjamin, 29, 73

Sridharan, Kumar, 66

Stewart, Frederick, 144

Sun, Xiaodong, 42

Szlufarska, Izabela, 176

$\mathbf{T}$

Teague, Melissa, 2

Terry, Jeff, 66
Teysseyre, Sebastien, 13

Thompson, Scott, 6, 99, 101, 110

Thornton, Katsuyo, 26

Tonks, Michael, 2, 10, 18, 26

Turk, Bob, 153

Tyburska-Püschel, Beata, 66

W

Wahlen, Brad, 193

Wang, Yaqi, 37

Warren, Ian, 204

Wasden, Daryl, 84, 85

Watson, Scott, 99, 101

Welhan, John, 204

West, Shawn, 87

Williamson, Richard, 18, 26, 54, 61

Wilson, Aaron, 144

Wu, Yaqiao, 197

$\mathbf{Y}$

Yang, Yong, 182

Yildiz, Bilge, 167

Youngblood, Robert, 57, 180

Z

Zalupski, Peter, 35, 171, 174

Zarzana, Chris, 20, 79, 161

Zhang, Hongbin, 37, 73

Zhang, Yongfeng, 10, 26, 51 
APPENDICES 


\section{Appendix A}

\section{Commonly Used Acronyms}

The following acronyms are used in many of the project descriptions.

ATR

BISON

CAES

DOE-NE

FY

INL

LDRD

MARMOT

MARVEL

MFC

MOOSE

NSUF

PI

$R \& D$

RELAP5
Advanced Test Reactor

Thermo-mechanical code that models nuclear fuel performance at the engineering scale

Center for Advanced Energy Studies

Department of Energy Office of Nuclear Energy

fiscal year

Idaho National Laboratory

Laboratory Directed Research and Development

Mesoscale Model Application Computer Code

Multiplying Assembly for Research, Validation, Evaluation, and Learning

Materials and Fuels Complex

Multiphysics Object Oriented Simulation Environment

National Scientific User Facility

Principal Investigator

Research and Development

Thermal-Hydraulic Analysis Code 


\section{Appendix B}

\section{Relevance to DOE Program Offices}

\begin{tabular}{|c|c|c|c|c|c|c|c|c|c|c|c|}
\hline $\begin{array}{l}\text { Tracking } \\
\text { No. }\end{array}$ & PI Name & Title & $\varangle$ & w & 端 & щu & u & $\mathbf{u}$ & $\frac{\alpha}{z}$ & Ш & $\Sigma$ \\
\hline $12-001$ & Kurt Derr & $\begin{array}{l}\text { Long Term Evolution Wireless Cyber } \\
\text { Security }\end{array}$ & $S$ & & & & $P$ & S & & $S$ & \\
\hline $12-026$ & Xianming Bai & $\begin{array}{l}\text { Protectiveness and stability of the zirconium } \\
\text { oxide in early-phase corrosion of zirconium } \\
\text { alloys - predictive relations to surface } \\
\text { structure and composition }\end{array}$ & & & & & $S$ & $P$ & & & \\
\hline $12-031$ & Melissa C. Teague & $\begin{array}{l}\text { 2-D and 3-D EBSD Technique } \\
\text { Development and Microstructure } \\
\text { Reconstruction for Phase Field } \\
\text { Microstructure Evolution Models }\end{array}$ & & & & & & $P$ & & & \\
\hline $12-056$ & Peter Zalupski & $\begin{array}{l}\text { Recovery of Precious Metals from Used } \\
\text { Nuclear Fuel and Electronic Waste Using } \\
\text { Sulfur-Impregnated Nanoscaffolds }\end{array}$ & & & S & & & $P$ & & & \\
\hline $12-059$ & R. Scott Herbst & $\begin{array}{l}\text { Chemistry and Flowsheet Development for } \\
\text { Lanthanide Separations }\end{array}$ & & $S$ & $S$ & $S$ & $S$ & $P$ & $S$ & & \\
\hline $12-061$ & Robert Mariani & $\begin{array}{l}\text { Development of a High Burn up Zr Based } \\
\text { Fuel Cladding for LWRs }\end{array}$ & & & & & $S$ & $P$ & & & \\
\hline $12-062$ & David L. Chichester & $\begin{array}{l}\text { Development of Diamond Based Sensors } \\
\text { for User Facility Capabilities at INL } \\
\text { Advanced Test Reactor }\end{array}$ & $S$ & & & & $S$ & $\mathrm{P}$ & $S$ & & \\
\hline $12-070$ & J. Keith Jewell & $\begin{array}{l}\text { Separate Effects Fuels Development } \\
\text { Capability }\end{array}$ & $S$ & $S$ & & & $S$ & $P$ & & & \\
\hline $12-077$ & Marat Khafizov & $\begin{array}{l}\text { Observation of zirconium oxidation at } \\
\text { atomic level using non-linear optical } \\
\text { spectroscopy }\end{array}$ & & & & & $S$ & $\mathrm{P}$ & & & \\
\hline $12-086$ & Ronald L. Boring & Alert/Alarm Dashboard & & & & & $S$ & $\mathrm{P}$ & & $S$ & \\
\hline $12-093$ & Robert V. Fox & $\begin{array}{l}\text { Development of Unique Nanomaterials for } \\
\text { Selective Replacement of Strategic and } \\
\text { Critical Materials }\end{array}$ & & $\mathrm{P}$ & & & $S$ & & & & \\
\hline $12-113$ & Hai Huang & $\begin{array}{l}\text { Coupled-Smoothed Particle Hydrodynamics } \\
\text { and Discrete Element Model (SPH-DEM) } \\
\text { Simulation of Hydraulic Fracturing and } \\
\text { Shale Gas Production }\end{array}$ & & & S & $P$ & S & & & & \\
\hline $13-011$ & Deborah T. Newby & $\begin{array}{l}\text { Integrated Approach to Algal Biofuel, Bio- } \\
\text { power, and Agricultural Waste Management }\end{array}$ & & $\mathrm{P}$ & & & & & & & \\
\hline
\end{tabular}




\begin{tabular}{|c|c|c|c|c|c|c|c|c|c|c|c|}
\hline $\begin{array}{l}\text { Tracking } \\
\text { No. }\end{array}$ & PI Name & Title & $\varangle$ & ш & 绎 & ш & u & $\mathbf{z}$ & $\frac{\alpha}{z}$ & 山ै & $\sum$ \\
\hline $13-013$ & S. Bulent Biner & $\begin{array}{l}\text { Development a micromechanistic phase- } \\
\text { field modeling approach for life estimation } \\
\text { and risk assesment of reactor pressure } \\
\text { vessels }\end{array}$ & & & & & $S$ & $P$ & $S$ & & \\
\hline $13-027$ & Eric J. Dufek & $\begin{array}{l}\text { Diagnostics of Advanced Energy Storage } \\
\text { Materials }\end{array}$ & & $P$ & & & $S$ & & & & \\
\hline $13-029$ & Joseph Palmer & $\begin{array}{l}\text { In-Pile Detection of Crack Growth in the } \\
\text { ATR }\end{array}$ & & & & & & $\mathrm{P}$ & $S$ & & \\
\hline $13-032$ & Michael R. Tonks & $\begin{array}{l}\text { Experimental and Computational Analysis } \\
\text { of Hydride Microstructures in Zirconium in } \\
\text { Dry Storage Conditions }\end{array}$ & & & & & $\mathrm{S}$ & $P$ & & & \\
\hline $13-033$ & Leigh R. Martin & $\begin{array}{l}\text { Magnetic Separation Nanotechnology for } \\
\text { Spent Nuclear Fuel Recycle }\end{array}$ & & & & & & $P$ & & & \\
\hline $13-035$ & Ken Bateman & $\begin{array}{l}\text { Development of New Molten Salt Sensor } \\
\text { Technology for Application to Safeguarding } \\
\text { Pyroprocessing }\end{array}$ & $P$ & & & & & $S$ & & & \\
\hline $13-039$ & Gary S. Groenewold & $\begin{array}{l}\text { Induction Based Fluidics Mass } \\
\text { Spectrometry for Characterizing } \\
\text { Radioactive Extraction Solvents }\end{array}$ & & & & & $S$ & $P$ & & & \\
\hline $13-040$ & Joseph Nielsen & $\begin{array}{l}\text { Epistemic Uncertainty Quantification Within } \\
\text { Dynamic Probabilistic Risk Assessment }\end{array}$ & & & & & & $\mathrm{P}$ & $\mathrm{S}$ & & \\
\hline $13-050$ & $\begin{array}{l}\text { Yongfeng Zhang } \\
\text { (Mike Tonks) }\end{array}$ & $\begin{array}{l}\text { Concurrent atomistic to macroscale } \\
\text { modeling of materials under irradiation } \\
\text { using the phase field crystal model }\end{array}$ & $S$ & & & & $S$ & $P$ & $\mathrm{~S}$ & & \\
\hline $13-060$ & James E. Delmore & $\begin{array}{l}\text { Metal Fluoride Preparation for AMS } \\
\text { Analysis }\end{array}$ & $P$ & & & & & & & & \\
\hline $13-065$ & Humberto E. Garcia & $\begin{array}{l}\text { Multi-domain Modeling, Simulation, and } \\
\text { Integration Tools for the Dynamic Analysis } \\
\text { and Optimization of Hybrid Energy Systems }\end{array}$ & & $P$ & & $S$ & $S$ & $S$ & & $S$ & \\
\hline $13-068$ & Mitchell Plummer & $\begin{array}{l}\text { Cooling in Fractured Geothermal } \\
\text { Reservoirs: Analyses of long-term cooling in } \\
\text { typical geothermal reservoirs and } \\
\text { application to geothermal resource potential } \\
\text { assessments }\end{array}$ & & $\mathrm{P}$ & & & $S$ & & & & \\
\hline $13-071$ & Benjamin Spencer & $\begin{array}{l}\text { Advanced Fracture Modeling for Nuclear } \\
\text { Fuel }\end{array}$ & & & & & & $P$ & & & \\
\hline $13-079$ & Dayna L. Daubaras & $\begin{array}{l}\text { Diverse Biological Factories for Sustainable } \\
\text { Manufacturing }\end{array}$ & & $\mathrm{P}$ & & & $\mathrm{S}$ & & & & \\
\hline 13-092 & Jana Pfeiffer & Fission Product Standard Production & $\mathrm{P}$ & & & & & & & & \\
\hline $13-093$ & Hussein Moradi & $\begin{array}{l}\text { Spectrum Allocation and Communications } \\
\text { in Dynamic Spectrum Access Channels }\end{array}$ & $S$ & & & & $S$ & $S$ & & $\mathrm{P}$ & \\
\hline
\end{tabular}




\begin{tabular}{|c|c|c|c|c|c|c|c|c|c|c|c|}
\hline $\begin{array}{l}\text { Tracking } \\
\text { No. }\end{array}$ & PI Name & Title & $\varangle$ & ш & 泣 & ш & u & $\mathbf{z}$ & $\frac{\alpha}{z}$ & Ш & $\sum$ \\
\hline $13-095$ & Robert Youngblood & $\begin{array}{l}\text { Development and Validation of a Societal- } \\
\text { Risk Goal for Nuclear Power Plant Safety }\end{array}$ & $S$ & & & & & $P$ & & & \\
\hline $13-097$ & Derek Gaston & $\begin{array}{l}\text { MOOSE Capability Extension In Support of } \\
\text { Full Core Modeling }\end{array}$ & & & $S$ & & $\mathrm{~S}$ & $P$ & $S$ & & \\
\hline $13-105$ & Jian Gan & $\begin{array}{l}\text { Micro/Nano Scale AFM-based Thermal } \\
\text { Conductivity Measurement and Atomistic } \\
\text { Modeling for Oxide Fuel: the effects of grain } \\
\text { boundary, fission gas and radiation damage }\end{array}$ & & & & & & $P$ & & & \\
\hline $13-106$ & Peter Zalupski & $\begin{array}{l}\text { Building Organic-Inorganic Hybrid Materials } \\
\text { to Protect Metal lon Sequestering Agents } \\
\text { from Radiation Induced Oxidative Damage }\end{array}$ & $S$ & & $S$ & & & $P$ & & & \\
\hline $13-110$ & Humberto Garcia & $\begin{array}{l}\text { Nuclear-Renewables-Oil Shale Hybrid } \\
\text { Energy System }\end{array}$ & & $S$ & & $S$ & & $P$ & & $S$ & \\
\hline $13-114$ & Earl D. Mattson & $\begin{array}{l}\text { Multiphase Fluid Trapping in Propped } \\
\text { Hydraulic Fractures }\end{array}$ & & & $S$ & $\mathrm{P}$ & $\mathrm{S}$ & & & & \\
\hline $13-115$ & Mark DeHart & $\begin{array}{l}\text { Multi-scale Full Core Reactor Physics } \\
\text { Simulation }\end{array}$ & & & & & & $\mathrm{P}$ & $S$ & & \\
\hline $13-118$ & Shawn West & GMD Field Coupling Measurements & $S$ & & & & & & & $\mathrm{P}$ & \\
\hline $13-121$ & $\begin{array}{l}\text { J. Keith Jewell } \\
\text { (previously Tony Hill) }\end{array}$ & $\begin{array}{l}\text { Advanced In-Situ Measurement Techniques } \\
\text { in TREAT }\end{array}$ & $S$ & & $S$ & & $S$ & $P$ & & & \\
\hline $14-009$ & James E. O'Brien & $\begin{array}{l}\text { Development of an INL Capability for High } \\
\text { Temperature Flow, Heat Transfer, and } \\
\text { Thermal Energy Storage with Applications } \\
\text { in Advanced Small Modular Reactors, High } \\
\text { Temperature Heat Exchangers, Hybrid } \\
\text { Energy Systems, and Dynamic Grid Energy } \\
\text { Storage Concepts }\end{array}$ & & $S$ & & & & $\mathrm{P}$ & & $S$ & \\
\hline $14-010$ & Knudson, Darrell & $\begin{array}{l}\text { Use of Linear Variable Differential } \\
\text { Transformer (LVDT)-Based Methods to } \\
\text { Detect Real-Time Geometry Changes } \\
\text { during Irradiation Testing }\end{array}$ & & & & & & $P$ & $S$ & & \\
\hline $14-025$ & Greenhalgh, Mitchell & $\begin{array}{l}\text { Minor Actinide and Lanthanide Separations } \\
\text { in Alternative Media }\end{array}$ & & & $S$ & & $\mathrm{~S}$ & $P$ & & & \\
\hline $14-026$ & Yongfeng Zhang & $\begin{array}{l}\text { Multiscale modeling on delayed hydride } \\
\text { cracking in zirconium: hydrogen transport } \\
\text { and hydride nucleation }\end{array}$ & & & & & $S$ & $P$ & & & \\
\hline $14-031$ & Williamson, Richard & $\begin{array}{l}\text { Multidimensional Multiphysics Modeling of } \\
\text { Fuel Behavior During Accident Conditions }\end{array}$ & & & & & & $P$ & & & \\
\hline $14-032$ & Jonathon Chugg & $\begin{array}{l}\text { CAN Bus Security Across Multi-Sector } \\
\text { Platforms }\end{array}$ & & $S$ & & & & & & $P$ & \\
\hline $14-035$ & Michael Daniels & $\begin{array}{l}\text { Safer Energetic Materials - Ignition } \\
\text { Prevention with Improper Heating Rate }\end{array}$ & & & & & $\mathrm{P}$ & & & & \\
\hline
\end{tabular}




\begin{tabular}{|c|c|c|c|c|c|c|c|c|c|c|c|}
\hline $\begin{array}{l}\text { Tracking } \\
\text { No. }\end{array}$ & PI Name & Title & $\varangle$ & 岃 & 端 & ш & u & $\mathbf{u}$ & $\frac{\alpha}{z}$ & Ш & $\sum$ \\
\hline $14-036$ & David L. Chichester & $\begin{array}{l}\text { Innovative Research for Fieldable Nuclear } \\
\text { Measurements }\end{array}$ & $P$ & & S & & $S$ & $S$ & & & \\
\hline $14-037$ & David L. Chichester & $\begin{array}{l}\text { Development of Advanced Nuclear Material } \\
\text { Characterization Technology for Security } \\
\text { Applications }\end{array}$ & $P$ & & S & & $S$ & $S$ & & & \\
\hline $14-038$ & Curtis Smith & $\begin{array}{l}\text { Multivariate Calibration of Complex } \\
\text { Simulation Codes Using Disparate Types of } \\
\text { Evidence }\end{array}$ & & & & & & $P$ & & & \\
\hline $14-041$ & Paul A. Lessing & $\begin{array}{l}\text { Uranium Nitride - Uranium Silicide } \\
\text { composite ceramic fuel production via } \\
\text { Spark Plasma Sintering }\end{array}$ & & & & & & $P$ & & & \\
\hline $14-042$ & Mathew T. Kinlaw & Treaty Relevant Modeling & $P$ & & & & & & & & \\
\hline $14-044$ & Leigh R. Martin & $\begin{array}{l}\text { Development of actinide halide and oxy- } \\
\text { halides for isotopic separations }\end{array}$ & $P$ & & $S$ & & $S$ & $S$ & $S$ & & $S$ \\
\hline $14-045$ & Scott J. Thompson & $\begin{array}{l}\text { End-to-End Radiation Detector } \\
\text { Enhancements for Improved Safety and } \\
\text { Security in Safeguarded Facilities }\end{array}$ & $P$ & & & & $S$ & $S$ & & & \\
\hline $14-075$ & Cristian Rabiti & $\begin{array}{l}\text { Developments of tools and methodologies } \\
\text { for uncertainties quantification and } \\
\text { validation for the multi physics fuel } \\
\text { performance simulation }\end{array}$ & & & & & & $P$ & & & \\
\hline $14-078$ & Laura Carroll & $\begin{array}{l}\text { Extended Stability Gamma-Gamma Prime } \\
\text { Containing Nickel-Base Alloys }\end{array}$ & & $P$ & & $S$ & & $S$ & & $S$ & \\
\hline $14-079$ & Aaron D. Wilson & $\begin{array}{l}\text { Second Generation Switchable Polarity } \\
\text { Solvent Draw Solutes for Forward Osmosis }\end{array}$ & & $\mathrm{P}$ & $S$ & $S$ & $S$ & $S$ & & & \\
\hline $14-080$ & Dennis C. Kunerth & $\begin{array}{l}\text { Battery Material Characterization } \\
\text { Technologies }\end{array}$ & & $P$ & & & & & & & \\
\hline $14-086$ & Kurt Myers & $\begin{array}{l}\text { Development of a Mircogrid/Smartgrid } \\
\text { Testbed for ESL and Super Lab Initiative } \\
\text { with Load variability characterization and } \\
\text { control for renewable energy integration }\end{array}$ & $S$ & $\mathrm{P}$ & $S$ & $S$ & & $S$ & $S$ & $S$ & \\
\hline $14-087$ & Thomas Lillo & $\begin{array}{l}\text { Transparent Fiber Reinforcements for } \\
\text { Transparent Protection Systems }\end{array}$ & $P$ & & & & & & & & \\
\hline $14-090$ & Hussein Moradi & $\begin{array}{l}\text { SWANS: Secure Wireless Sensor } \\
\text { Networks for Smart Grid Communications }\end{array}$ & $S$ & $S$ & & & $S$ & $S$ & & $P$ & \\
\hline $14-091$ & Sergiy Sazhin & $\begin{array}{l}\text { Battery health estimation based on self- } \\
\text { discharge fast measurement and soft short- } \\
\text { circuit detection }\end{array}$ & & $P$ & & & $S$ & & & $S$ & \\
\hline $14-093$ & Ryan Hruska & $\begin{array}{l}\text { All Hazards Critical Infrastructure } \\
\text { Knowledge Framework }\end{array}$ & & $S$ & & & & & & $\mathrm{P}$ & \\
\hline $14-094$ & Michael Bakas & $\begin{array}{l}\text { SMC Advanced Arrmor Materials and } \\
\text { Systems R\&D }\end{array}$ & $P$ & & & & $S$ & & & $S$ & \\
\hline
\end{tabular}




\begin{tabular}{|c|c|c|c|c|c|c|c|c|c|c|c|}
\hline $\begin{array}{l}\text { Tracking } \\
\text { No. }\end{array}$ & PI Name & Title & $\varangle$ & 岃 & $\sum_{\text {W }}$ & ш & u & W & $\frac{\alpha}{z}$ & 山ै & $\sum$ \\
\hline 14-095 & Gary S. Groenewold & $\begin{array}{l}\text { In Situ Measurement of Electrolyte } \\
\text { Chemistry in Battery Cells During Operation }\end{array}$ & & $P$ & & & & & & $S$ & \\
\hline $14-098$ & Jian Gan & Irradiation Effects of Uranium Dioxide & & & & & $\mathrm{S}$ & $P$ & & & \\
\hline $14-102$ & Justin Coleman & Advanced Seismic PRA & $S$ & & S & & & $\mathrm{P}$ & & & \\
\hline $14-103$ & Craig Rieger & $\begin{array}{l}\text { Grid-centric Demonstration of Resilience } \\
\text { Technologies }\end{array}$ & & $\mathrm{S}$ & & $S$ & & $S$ & & $\mathrm{P}$ & \\
\hline $14-104$ & Richard C. Martineau & $\begin{array}{l}\text { Development of a Multiphysics Algorithm for } \\
\text { Analyzing the Integrity of Nuclear Reactor } \\
\text { Containment Vessels Subjected to Extreme } \\
\text { Thermal and Overpressure Loading } \\
\text { Conditions }\end{array}$ & & & & & $\mathrm{S}$ & $P$ & $S$ & & \\
\hline $14-106$ & Joshua J. Kane & $\begin{array}{l}\text { Understanding the Growth of Ultra-long } \\
\text { Cabon Nanotubes }\end{array}$ & & $\mathrm{P}$ & $S$ & & & $S$ & & $S$ & \\
\hline
\end{tabular}

